


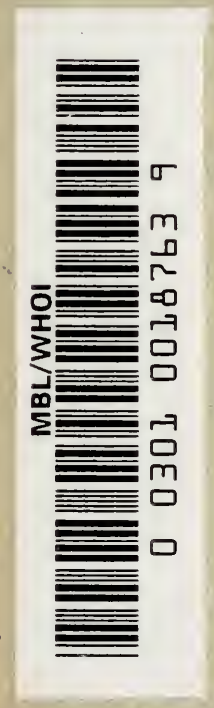



A COURSE OF INSTRUCTION

IN

Z O O T O M.

(VERTEBRATA.) 
s. 


\section{A COURSE OF INSTRUCTION}

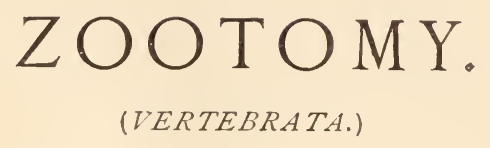

BY

T. JEFFERY PARKER, B.SC. LOND.

PROFESSOR OF BIOLOGY IN THE UNIVERSITY OF OTAGO. NEW ZEALAND.

WITH SEVENTY-FOUR ILLUSTRATIONS.

कondon:

M A C I L L A N A D CO.

I 884 . 
LONDON:
R. CLAsS, SONS, AND TAYLOR,
BREAD STREET hiLL, E C.

3305 
MY FRIEND AND CRITIC,

C. E. R. P.,

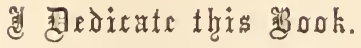





\section{PREFACE.}

THE directions for the dissection of certain vertebrate animals of which the present book consists were begun many years ago when I was privileged to act as demonstrator to Professor Huxley, at the Royal School of Mines, South Kensington. They are drawn up on much the same plan as the "Laboratory Work," in Professors Huxley and Martin's Elementary Biology', and aim at being a continuation of the zoological part of that work, in much the same way as the Practical Physiology of Dr. Foster and Mr. Langley may be said to continue it on the physiological side.

It must be borne in mind that the book is merely a "Course of Instruction," in the dissection of Vertebrates, and that it makes no pretence whatever at giving a complete account of the anatomy of the types selected. The time at the disposal of the average student of Comparative Anatomy is so short, that for him to examine half a dozen vertebrate animals - to say nothing of Invertebrates-in the same detail as the student of Human Anatomy examines his single type, would be quite out of the question even if it were necessary. On the other hand, it is of the first importance that he should 
not only be made familiar with the essentials of vertebrate organisation, but should be so drilled in details as to be capable of working out, with some degree of thoroughness, any ordinary vertebrate animal which may be set before him.

The advantage of the study of Comparative over that of Human Anatomy lies in the fact that in the former the dry facts are, to borrow an expression of Dr. Michael Foster's, "salted with the salt of morphological ideas." But if the same thoroughness in practical work is not demanded of the student of Animal Morphology as of the medical student, if he is allowed to shirk the discipline of laborious dissection, and to be content with a "general notion" of the structure of the types he examines, he runs a very serious risk of getting uncommonly little meat to his salt, and of losing in depth what he gains in breadth.

It was therefore necessary to decide how much might be left out without leading the student into a superficial way of working; and in striving to "keep the mean between the two extremes of too much stiffness in refusing and of too much easiness in allowing" any omissions, I am only too well aware how largely the personal equation enters into questions of this sort, and how unsatisfactory the compronise I have decided upon may seem to other teachers.

The subjects described are mostly such as can be readily obtained at any time of the year. The Skate is chosen in preference to the more typical Dogfish, partly because it is 
a regular market commodity, partly because it is, to my mind, quite unrivalled for the study of the vertebrate nervous system. The Rabbit is chosen in preference to the Dog or Cat-either of which, and especially the former, would have been preferable for some reasons-because it can be more readily obtained in quantity; its size, moreover, makes it more suitable than the Rat. The Green Lizard and the Lamprey cannot always be depended on, but, as they can both be easily preserved whole in alcohol, this is of less consequence than in the other cases. It was not thought necessary to include an amphibian, since the Frog is fully described in the Elementary Biology.

The labour of correcting the proofs and of superintending the drawing on wood and engraving of the illustrations has fallen upon my brother, Mr. W. N. Parker, F.Z.S., without whose help the publication would have been almost out of the question. Besides the editorial work, he has made out several points in the anatomy of the Cod and Lizard, which, owing to lack of material, I was unable to decide myself. Many important omissions and some actual errors have thus been avoided.

I am indebted to Professor Huxley for having kindly read over such parts of the work as were written when I left England, and for valuable suggestions thereon. But beyond this, I shall always feel that any value the book may possess is very largely due to the example set me by Professor Huxley during all the years I had the good fortune to be associated with him. 
My friend and former colleague, Mr. G. B. Howes, rendered me much assistance in preparing and correcting those parts of the directions which were drawn up in England, and has also given considerable aid to my brother in working out some doubtful points which arose while the work was passing through the press.

I am further indebted to Mr. Howes for the drawing for Fig. 34, and to my brother for Fig. 32. Figs. 35, 36, and 37 are copied from my father's monograph on the Lizard's skull (Phil. Trans. 1879), and Fig. 38 from Herpetologia Europaa; the remaining illustrations are from my own drawings. The whole of them have been drawn on the wood by Mr. Coombs, and engraved by Mr. Cooper : judging from the few proofs which have so far reached me, I have every reason to be grateful to those gentlemen for the care and skill with which their work has been done.

$$
\text { T. J. P. }
$$

Otago University Museum,

DUNEDIN, N.Z.

Fune, $188_{3}$. 


\section{CONTENTS.}

PREFACE . PAGE

LIST of IVOODCUTS . . . . . . . . . . xiii

INTRODUCTION . . . . . . . . . . . . XVii

THE LAMPREY-

A. The Skeleton . . . . . . . . . I

B. Directions for Dissection . . . . . . $S$

THE SKATE-

A. The Skeleton . . . . . . . . . 27

B. Directions for Dissection . . . . . . . 40

C. Special Dissection of the Nervous System and Sense Organs . . . . . . . . . . . 69

THE COD-

A. The Skeleton . . . . . . . . . . S6

B. Directions for Dissection . . . . . . . IOI

THE LIZARD-

A. The Skeleton . . . . . . . . . I30

B. Directions for Dissection . . . . . . . I53 
The Pigeon-

A. The Skeleton . . . . . . . . . . 182

B. Directions for Dissection . . . . . . 209

The Rabbit-

A. The Skeleton . . . . . . . . . . 262

B. Directions for Dissection . . . . . . . 286

WORKS OF REFERENCE . . . . . . . . . . 38 I

$\operatorname{INDEX} \cdot \cdot \cdot \cdot \cdot \cdot \cdot \cdot \cdot \cdot{ }_{3} S_{4}$ 


\section{LIST OF WOODCUTS.}

FIr. PAGE

I. Petromyzon marinus. The skull, from the left side ... . 3

2. The brain-case, from above . . . . . . . . . 4

3. The nasal capsule . . . . . . . . . . . 5

4. Dissection of the anterior part of the body, from the left side.............. . . . 9

5. The urinogenital sinus and adjacent parts . . . . . I I

6. Transverse section through the branchial region . . . . 12

7. Transverse section through the abdominal region . . . I4

S. Transverse section through the caudal region . . . . I7

9. The brain, from above . . . . . . . . . . 24

Io. Raja nasuta. The skeleton, from beneath . . . . . . jo

I I. Part of the vertebral column . . . . . . . . 3I

I2. A trunk vertebra, from the front . . . . . . . . 32

13. Longitudinal section of trunk vertebra . . . . . . 33

I4. A caudal vertebra, from the front . . . . . . . 34

I 5. The gall-bladder and bile-ducts . . . . . . . 49

I6. The portal vein .. . . . . . . . . . 5 I

I7. The venous system . . . . . . . . . . 53

IS. Raja batis. Urinogenital organs (male) . . . . . . . 55

I9. Urinogenital organs (female) . . . . . . . . 57

20. Raja nasuta. The arterial system . . . . . . . . . 62

21. Diagram of the heart and chief blood-vessels . . . . . 63

22. The nervous system, from above . . . . . . . . 7 I

23. Raja batis. The brain, from beneath . . . . . . . . . . 73

24. Longitudinal section of the brain . . . . . . . . . 74

25. The brain, from the left side . . . . . . . . . 74

26. Raja nasuta. Roots of the fifth, seventh, and eighth nerves 75 
FIG.

27. Raja nasuta. Principal branches of the fifth, seventh, and eighth nerves

28. Raja batis. The auditory organ

20. Gadus morrhua. Side view of a disarticulated skull

30. The superficial muscles and nerves . . . . . . . . . IO4

3. The alimentary canal . . . . . . . . . . Io8

32. The vascular system . . . . . . . . . . II7

33. The auditory organ ............ . . I 23

34. The brain . . . . . . . . . . . . . I 25

35. Lacerta agilis. Longitudinal section of the skull . . . . . I37

36. The columell $九$ auris . . . . . . . . . . . I 42

37. The chondrocranium, from above. . . . . . . . . I44

38. $\left\{\begin{array}{l}\text { Lacerta viridis. } \\ \text { Lacerta agitis. } \\ \text { Zootoca vivipara. }\end{array}\right\} \begin{aligned} & \text { Outline of the head in the three species, } \\ & \text { showing the epidermic scales . . . . I55 }\end{aligned}$

39. Lacerta viridis. Muscles of the trunk, ventral aspect . . . I57

40. Dissection from the ventral aspect, showing the alimentary canal and vascular system . . . . . . . I 60

4. Urinogenital organs (male) . . . . . . . . . I66

42. Urinogenital organs (female) . . . . . . . . . . I67

43. The heart, dorsal aspect . . . . . . . . . I72

44. The aortic arches . . . . . . . . . . . I73

45. The brain . . . . . . . . . . . . . . . . I77

46. Columba livia. Sacrum of a young individual . . . . . . IS7

47. Skull of a young individual . . . . . . . . . . . . I 89

48. The columella auris . . . . . . . . . . . . . . 195

49. Manus of a young individual . . . . . . . . . . 203

50. Pelvis of a young individual . . . . . . . . . . 204

51. Tarso-metatarsus of an unhatched embryo . . . . . 207

52. Urinogenital organs (male) . . . . . . . . . 228

53. Urinogenital organs (female) . . . . . . . . . 230

54. The circulatory system . . . . . . . . . 232

55. The cloaca . . . . . . . . . . . 236

56. The heart, from the dorsal aspect . . . . . . . . 24 I

57. The lungs and syrinx . . . . . . . . . 245

58. The brain . . . . . . . . . . . . 255

59. Dissections of the brain . . . . . . . . . . 259

6o. Lepus cuniculus. Part of the alimentary canal with the portal system and bile-ducts. . . . . . . 301

6r. The vascular system . . . . . . . . . . 309

62. Urinogenital organs (male and female), from the side . 317 
FIG. PAGiz

63. Lepus cuniculus. Part of the female organs, ventral aspect . 322

64. Longitudinal section of the head . . . . . . . . 334

65. Muscles of the fore limb-

A. From the outer side . . . . . . . . . 340

B. From the ventral aspect . . . . . . . 34I

C. From the inner side . . . . . . . . . 342

66. Muscles of the manus . . . . . . . . . . . 347

67. Muscles of the hind limb, inner side . . . . . . . . 352

68. Muscles of the hind limb, outer side . . . . . . . . 355

69. Muscles of the pes . . . . . . . . . . . $36 \mathbf{I}$

70. The brain, from beneath . . . . . . . . . . 367

71. Dissections of the brain, from above . . . . . . . . . 37 I

72. Longitudinal section of the brain . . . . . . 378 



\section{INTRODUCTION.}

Dissection is best performed at a strong deal table, not less than three feet long by two feet wide: it should, if possible, be placed at a window, so that the operator faces the light, otherwise the more delicate work will be hindered by the shadows of the hands and instruments.

In nearly every case the subject should be firmly fastened down during dissection, either to the table, or, better, to a soft deal board, about one foot and a half long by one foot wide, with a narrow bead tacked round the edge so as to convert it into a shallow tray: the advantages of this are that the subject may be turned in any direction without unfastening, and that the edge confines the mess caused by escape of blood, \&c., to a limited area. The best fastenings are small awls fixed into wooden handles, about an inch long, and just thick enough to be conveniently grasped: these can be more easily driven into the wood than large pins, are not so liable to bend, and hold more firmly. Care must of course be taken to thrust them through some 
part-e.g. skin-the slight injury to which will not interfere with the dissection.

The more delicate dissections are best conducted under water. A convenient dissecting dish is made by fitting a piece of sheet cork weighted with one of sheet lead into the bottom of a common pie-dish : the sloping sides of this latter are admirably adapted for admitting the greatest possible amount of light. The subject, in this case, is fixed out with small pins.

The most necessary dissecting instruments are :-

Three or four scalpels of various sizes.

A large and a small pair of scissors.

A large and a small pair of forceps.

A pair of bone-forceps.

A "seeker," i.e. a blunt bent needle fixed in a handle.

A. German silver anatomical blowpipe. ${ }^{1}$

The ordinary pointed form of scalpel is the best, those with obliquely truncated ends are rarely suitable. The scissors should have sharp points, and should bite well to the very end: this is especially important with the small pair, which will otherwise be perfectly useless. The forceps, also, should meet accurately at the points, which should be roughened so as to insure a firm grip; in the small pair the pin placed to prevent the points crossing when pressure is applied, should fit easily but not loosely in the hole for its

1 Boxes containing the above set of instruments are made by J. Weiss \& Son, 62, Strand; Hawkesley \& Son, 3co, Oxford Street; C. Baker \& Co., 244, High Holborn; J. Swift, 8r, Tottenham Court Road; and I. B. Medland, I2, Boro' High Street. 
reception; if the latter is too large the points will always be liable to cross.

The bone-forceps should have straight blades, and the handle should be sufficiently wide apart to admit of their being conveniently worked without obstruction by the fingers. They are used for cutting through bones; for instance, those of the skull in the removal of the brain.

The seeker is useful both as a probe and for clearing away the. connective tissue from blood-vessels, nerves, \&c., without danger of cutting them.

All the instruments should be of the best steel; it is false economy to buy inferior ones, as they lose their edge very quickly and require constant sharpening.

A useful addition to the above is a common butcher's knife for rough work, e.g. for cutting through the skin of the skate, the asperities of which completely spoil a scalpel. For fine dissection spring scissors are very useful, but are by no means indispensable.

Directions for injecting blood-vessels are given on $\mathrm{pp}$. 48, III 162, 2I8, and 298: to these I may add that a very convenient fluid injection for fine vessels is made by straining through muslin a strong solution of gum-arabic in water coloured with precipitated Prussian blue or carmine: after injection the subject is placed in alcohol, which coagulates the gum. This has the double advantage over gelatine (p. 162) that it is used cold and that it keeps better in alcohol.

Injecting syringes provided with brass cannulæ of various 
sizes are to be had from the instrument-makers. But a common brass ear-syringe holding about two ounces answers every purpose, using for cannulæ glass tubes of the form shown on p. 48 , adapted to the nozzle of the syringe with short pieces of caoutchouc tubing. "Bull-dog" forceps will be found very useful in injecting for clamping any vessels which may have been accidentally cut.

In large classes where the time of the student is limited, it is usually inconvenient for each one to inject his subject: it is then desirable to have an injected preparation in the room which may be consulted when necessary. For this purpose, a rough dissection which the students are allowed to handle is far more useful than a more elaborate preparation mounted in a bottle. In fact, one may say that the only alcoholic preparations of real use to beginners are those which are simple enough to tell their own tale almost at a glance. The most important, in my opinion, are :-

a. Preparations of the heart with the origins of the great vessels, either filled with solid injection, or distended with alcohol, the cavities, in the latter case, being cut open.

b. Preparations of the entire brain with the origins of the nerves, and various dissections of the same organ.

c. Preparations of the adult urinogenital organs, with the various accessory glands, ducts, apertures, \&c., displayed.

Many other very useful preparations might be mentioned, but the above, as demonstrating important points about which the beginner is certain to experience considerable difficulty, may be considered as the most essential. 
As the hearts and brains of most of the subjects selected are small, similar preparations from larger types are very valuable for demonstration; for instance, the monitor or iguana, the goose or turkey, and the dog, sheep, or even horse. It will, of course, be all the better if some of these structures-especially a larger mammalian brain than the Rabbit's - can be dissected by the students themselves as supplementary subjects.

Dissections of large hearts for demonstration may be prepared by the glycerine jelly process described on $\mathrm{p}$. $2 ;^{1}$ the various structures are more readily pointed out on such preparations than on those kept in alcohol: they may be made still clearer by colouring the different parts.

The larger brains are best prepared by Giacomini's method: the organ is placed in a saturated solution of zinc chloride, and then, after removal of the pia mater, in strong alcohol, which should be renewed at least once. When thoroughly hardened it is transferred to strong glycerine, and retained therein until thoroughly permeated: this is shown by its sinking in the fluid. The brain is then removed from the glycerine, drained thoroughly, and, after a time, sized and varnished. Any dissections which may be required are made before placing in glycerine.

${ }^{1}$ Since writing that description I find that the objects should be well hardened in alcohol before being placed in the glycerine fluid; that it is best to substitute 0.05 parts of a concentrated solution of phenol for the corrosive sublimate; and that the specimens are best finished by varnishing, after one or two coats of size, with common oak-varnish. 
During the dissection of any animal it is always advisable to have the skeleton at hand for reference, and at some time or other a systematic study should be made of it. It is for this reason that an account of the skeleton is prefixed to the directions for the dissection of each type.

With slight variations the management of the work is the same for all the subjects selected. The external characters are first studied: then a rough dissection is made for the purpose of acquainting the student with the position and general relations of the chief organs; at the conclusion of this stage the brain is removed and preserved for future examination; finally, the various parts are studied more or less in detail. 'The student should try and arrange his work so as to get the brain removed on the first day.

In the case of the Lamprey and the Lizard, which can be satisfactorily preserved whole in alcohol, it has not been thought necessary to remove the brain until it is wanted for dissection.

The directions for each stage in the dissection are given in " indented " paragraphs numbered with Roman numerals ; the following ordinary paragraphs with Arabic numerals giving descriptions of the structures brought into view by such dissection. By this arrangement the work is divided into sections, many of which may be omitted without serious disadvantage, if time runs short. The paragraphs in small type may also be omitted by the beginner.

When a dissection is prolonged over say two days, the subject should be placed, when not in use, in a preservative 
fluid, such as methylated spirit, 'Wickersheimer's fluid, ${ }^{1}$ or boro-glycerine.

I should strongly advise students to adopt the plan so much insisted upon by Professors Huxley and Martin in their Elementary Biology, of making sketches of their dissections. Even a rough drawing, if the various parts are properly named, and especially if they are further distinguished by different colours, forms a far better memorandum of work done than any mere description.

1 This fluid is made as follows : dissolve 100 grams of alum, 25 of common salt, 12 of saltpetre, 60 of potassic carbonate, and 20 of arsenious acid in 3 litres of boiling water, and add $\mathrm{I} \cdot 2$ litre of glycerine and $0^{*} 3$ litre of methylated alcohol. 



\section{ZOOTOM Y.}

\section{THE LAMPREY.}

The Sea Lamprey (Petromyzon marinus).

The Fresh-water Lamprey, or Lampern ( $P$. fluviatilis).

\section{A.-THE SKELETON.}

I. The preparation of the lamprey's skeleton is a very laborious process, owing to the extreme toughness of the connective tissue which invests it. Removal of the latter is assisted by maceration in nitric acid, ro per cent., care being taken not to allow the acid to act too long, the result of prolonged maceration being the entire separation of the cartilages of the skull proper, and the total destruction of the branchial basket. In preparing a skeleton for demonstration purposes, it is, however, advantageous to allow the cartilages to separate, and then to articulate them with fine platinum wire. The branchial basket can then be shown by itself in a special dissection. The 
skeleton may be either kept in spirit or prepared as follows :-

(a) Place for about three days in a solution composed of

Glycerine

ro parts.

Water

Corrosive sublimate

Alum

(b) Transfer to melted glycerine jelly made by dissolving 2 parts of gelatine, or "gelatine glue," in the above fluid : allow to remain for 2 to 4 days at a temperature just sufficient to keep the jelly fluid.

(c) Place in a dry room, until the surface no longer feels damp or sticky; then varnish with a solution of white (bleached) shellac in rectified spirit.

II. In the prepared skeleton make out the following points :-

I. The notochord (Fig. I, nc), a cylindrical rod, occupying the position of the vertebral column in one of the higher animals ; at its anterior end it tapers to a point somewhat suddenly; at its posterior end the tapering is more gradual. It is composed of a strong sheath of cartilage, inclosing a gelatinous central substance (Figs. 4, 6, 7, 8, nc).

2. The neural processes (Fig. I and 6 n.p), small rods of cartilage, set in pairs at short intervals along the dorso-lateral regions of the notochord, and partly inclosing the neural canal (Figs. 4 and $6-8, n . c a$ ), in which the spinal cord lies. Between the neural processes and completing the shutting-in of the neural canal both dorsally and laterally is a quantity of very tough pigmented fibrous tissue (Figs. 4, and $6-8, f . t)$. 


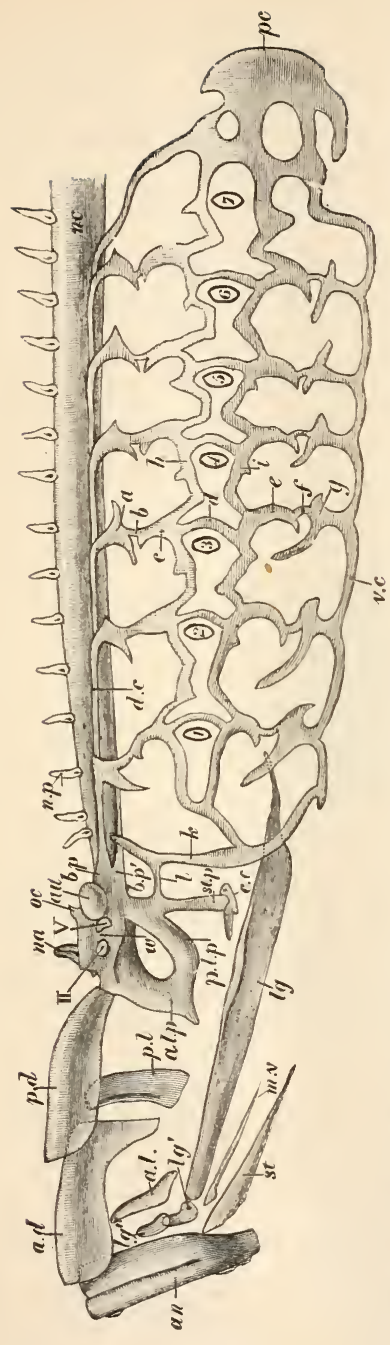

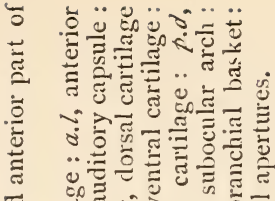

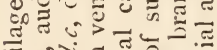

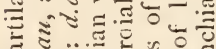

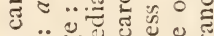

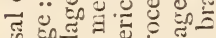

要

วั)

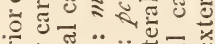

氜

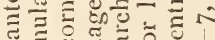

$\therefore$ 일 $\approx$

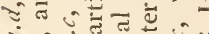

过记药

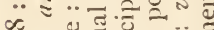

$\sim$ ․

-

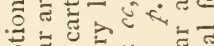

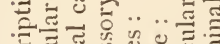

ट

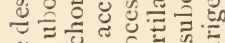

\&.

$\therefore \circ$ हैं

पै की

\%

㶽.

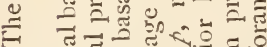
$\therefore \div$ 的... 出已.

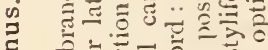

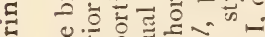

ส

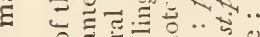

I $\circ$ 政

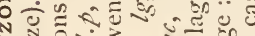

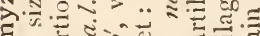

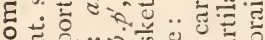

施

น 긍. 들 है

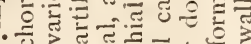

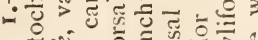

๕

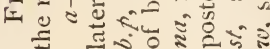


3. The fin-rays (Figs. 7 and 8, f.r), delicate cartilaginous filaments, supporting the dorsal and caudal fins $(\$ \$ 29,30)$. They are connected ventrally with the fibrous tissue covering in the neural canal, and lie parallel to one another and inclined backwards.

4. The brain-case, or hindmost division of the skull. Posteriorly it consists of a basal or parachordal plate (Figs. I and 2, b.p, b. $p^{\prime}$ ), surrounding the anterior end of the notochord, and divided by the latter into dorsal (b.p) and ventral $\left(b . p^{\prime}\right)$ portions. The ventral portion forms a continuous plate beneath the anterior end of the notochord (Figs. 2 and $4, b . p^{\prime}$ ); the dorsal portion consists of two

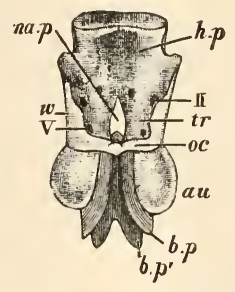

FIG. 2.-Petromyzon marinus. The brain case, from atove, the subocular arches supposed to be removed (nat, size : from a larger specimen than Fig. I).

$a u$, auditory capsules: $b \cdot p$, dorsal, and $b \cdot p^{\prime}$, ventral portion of basal or parachordal plate: h.p, hard palate: na.p, naso-palatine canal : $o c$, occipital arch : $t r$, trabecula : $w$, side wall : II, optic foramen : V, trigeminal foramen.

longitudinal plates, which do not unite over the notochord. Anteriorly the basal plate is continued forward into two flattened plates, the trabeculæ (Fig. 2, tr), which unite with one another in front, forming the hard palate ( $h . p$ ), and inclosing an oval space, the naso-palatine canal (na.p). Laterally, the trabecula and hard palate send up plates of cartilage $(w)$, which afford side walls to the brain- 
case, and unite with one another above the brain in a narrow band, the so-called occipital arch (o.c). The side walls are perforated with apertures for the exit of the optic (II), and of the fifth and seventh (V) nerves.

5. The auditory capsules (Figs. I and 2, au), paired oroidal masses of cartilage, fused with the basal plate and cranial walls, and each containing a cavity for the corresponding auditory sac $(\$ 95)$; their inner walls bound laterally the posterior part of the brain cavity, and are pierced with apertures for the auditory nerves.

6. The nasal capsule (Fig. I, na; Fig. 3), a concavoconvex plate of cartilage, of irregularly oval form, lying with its concave side forwards, in the posterior wall of the nasal sac $(\S 89)$, and forming the anterior boundary of the cerebral

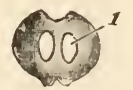

FIG. 3.-Petromyzon marinus. The nasal capsule, front view (nat. size). I, olfactory foramina.

cavity (see Fig. 4 na.c). Near its centre it is perforated by two oval apertures (Fig. 3, I) for the passage of the olfactory nerves.

7. The subocular arches, each consisting of an anterior lateral process (Fig. I, a.l.p), springing from the anterior end of the hard palate, and of a posterior lateral process (p.l.p), arising from the basal plate just beneath the auditory capsule. Both processes pass downwards, outwards, and forwards, and meet with one another at an acute angle.

8. The styliform processes (Fig. I, st.p), cylindrical rods springing one from the posterior edge of each posterior lateral process, near its proximal end, and taking a directly downward course. 
9. The cornual cartilages (Fig. I, c.c), small, irregular, horizontal pieces, connected by fibrous tissue to the distal ends of the styliform processes.

Io. The posterior dorsal cartilage (Figs. I and 4, p.d), an arched plate, bilobed anteriorly, and truncated posteriorly, where it enters into fibrous union with the anterior edge of the hard palate.

I I. The anterior dorsal cartilage (Figs. I and 4, $a . d)$, situated in front of and partly overlapped by the foregoing; it is strongly arched from side to side, ends in front in a thin rounded edge, and posteriorly is produced on each side into a downwardly directed process.

I2. The anterior lateral cartilages (Fig. I, a.l), situated one on either side, just in front of the descending process of the anterior dorsal cartilage; their form is somewhat sigmoidal.

I3. The posterior lateral cartilages (Fig. I, p.l), flattened plates, situated one on either side beneath the posterior dorsal cartilage, and between the descending process of the anterior dorsal cartilage, and the anterior lateral process of the subocular arch.

I4. The lingual cartilage (Figs. I and 4, $7 \mathrm{~g}$ ), a long median ventral piece, flattened from side to side, truncated in front, and tapering posteriorly; by its anterior end it is united by fibrous tissue to a small cartilage $\left(l g^{\prime}\right)$ having the form of a semicircle, and set transversely: with the free, upwardly directed ends of this are connected two small irregular cartilages $\left(l g^{\prime \prime}\right)$. The lingual cartilage lies in the floor of the mouth; the semicircular cartilage $\left(l g^{\prime}\right)$ supports the median portion of the tongue; the small cartilages $\left(\lg ^{\prime \prime}\right)$ lie in the lateral wings of the tongue $\left(\begin{array}{l}\S \\ 2\end{array}\right.$, Fig. $\left.4, t, t^{\prime}\right)$.

I5. The median ventral cartilage (Figs. I and 4, 
m. $v^{\prime}$ ), a slender rod, having the form of a $\mathrm{T}$ with a very long stem, and situated in the middle line beneath the lingtial cartilage, the anterior ends of the two being connected by fibrous tissue.

I6. The annular cartilage (Figs. I and 4 , an), a stout circular cartilage, supporting the oral funnel ( $\$ 2 \mathrm{I}$ ), and giving attachment to the maxillary and mandibular teeth $(\$ 24)$.

17. The styliform cartilages (Fig. I, st), two elongated, tapering rods, attached by their thickened proximal ends to the posterior edge of the annular cartilage, a little below its middle, and directed backwards and somewhat downwards.

I 8. The branchial basket (Fig. I, d.c, v.c, $a-k, p c$ ), a cartilaginous framework supporting the gills. It consists essentially, on each side, of (I) a longitudinal rod or dorsal cartilage $(d . c)$, springing from the dorsal portion of the parachordal (b.p), and passing backwards along the side of the notochord; (2) a second longitudinal rod or ventral cartilage $(v . c)$, lying close alongside, and partly fused with, its fellow of the opposite side, in the middle ventral line; (3) irregular transverse arches $(a-s, k)$ uniting the dorsal and ventral cartilages and passing between the outer gillclefts; (4) lateral longitudinal bars $(h, i)$ connecting the transverse arches immediately above and immediately below the gill-clefts; and (5) a cartilage ( $p c)$ supporting the posterior and lateral walls of the pericardium.

Each of the transverse arches, except the first and the eighth or last, consists of seven well-marked portions; of these, the first or dorsalmost $(a)$ and the seventh or ventralmost $(g)$ are flattened plates, produced into longer or shorter anterior and posterior processes; the second $(b)$ and sixth $(f)$ are short curved rods; the third $(c)$ and ffth (e) are flattened, and are produced into forwardly directed processes; the fourth or middle portion $(d)$ is a curved rod situated immediately 
behind the corresponding gill-cleft. The longitudinal connecting bars $(h$ and $i$ ) are flattened, and unite with the third $(c)$ and the fifth $(e)$ segments of the transverse arches respectively. In front of the first gillcleft the two longitudinal connecting bars run together and form a single rod, which becomes connected with the first transverse arch $(k)$. This latter is free at its dorsal end, of tolerably regular cylindrical form, and united by a short rod of cartilage with the proximal end of the styliform process (st.p). The eighth transverse arch is also regularly cylindrical at its dorsal and ventral ends, but in the middle of its course becomes fused with the hinder ends of the longitudinal connecting bars and with the pericardial cartilage $(p c)$. The latter has the form of a backwardly directed hemispherical cup, supporting the posterior wall of the pericardium, and produced into dorsal, ventral, and lateral processes, of which the last enter into union with the branchial basket proper, as already described.

The cartilages of the branchial basket lie, for the most part, immediately beneath the dorsal and ventral muscles covering the gills ( $\$ 34$, Fig. $6, h, i)$, so that they are seen at once when those muscles are removed: but the pieces marked $b, d$, and $f$, are curved inwards and are consequently not seen until the surrounding tissues are dissected away. ${ }^{1}$

\section{B.-Directions for Dissection.}

III. Verify the following external characters :-

19. The elongated vermiform body, almost circular in section anteriorly, but becoming compressed from side to side posteriorly; the head passes insensibly into the trunk, and the trunk into the tail.

20. The integument, smooth and slimy, and entirely devoid of scales: marbled with black in P. marinus, of a uniform bronze-green tint in $\mathrm{P}$. fluviatilis.

21. The sucker-like oral funnel (Fig. 4, o.f) at the anterior end of the body, inclined obliquely downwards and forwards: its rim, which is beset with numerous vascular papillæ, passes insensibly into the general surface of the

${ }^{1}$ This description of the branchial basket is taken from a single specimen of P. marinus--the only one at my disposal. 


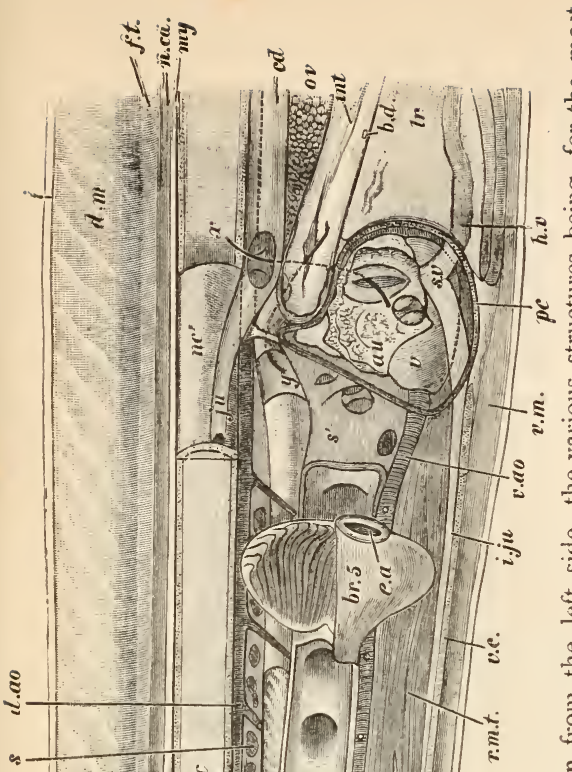

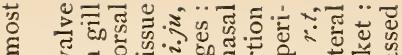

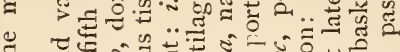

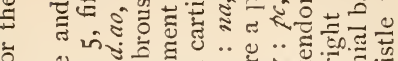

b ond

to

का

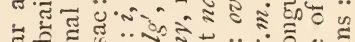
(1)

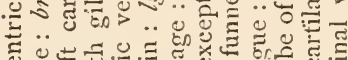

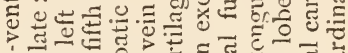

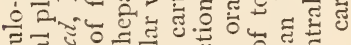

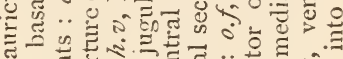

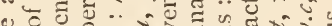

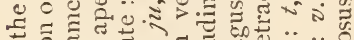

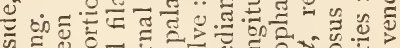

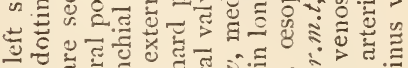
00 \% 둘

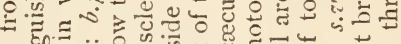
5. द. 언

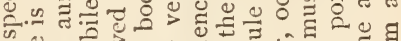
을

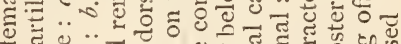

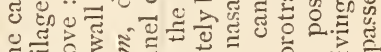

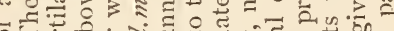
कुत 후의.

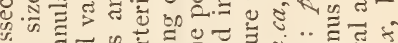

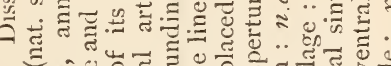

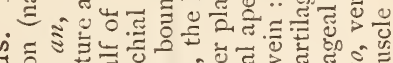

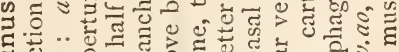

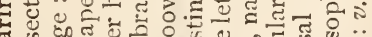

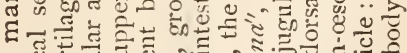
등 80

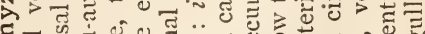
E

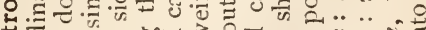
年 1 on

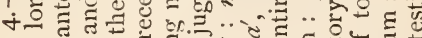

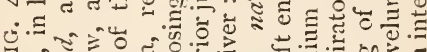

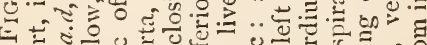

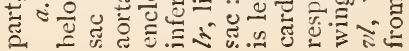


body above and at the sides, while below it is separated by a deep furrow (Fig. 4, $g r$ ).

22. The mouth (Fig. 4, $m$ ), situated at the bottom of the oral funnel, and of a somewhat crescentic form.

23. The tongue (Fig. 4, t), the end of which forms a well-marked protuberance bounding the mouth below, and produced on either side into an upwardly directed, wing-like process $(t)$.

24. The yellow horny teeth, which beset the inner surface of the oral funnel and the tip of the tongue: one immediately above the opening of the mouth is large and bifid, and is distinguished as the maxillary tooth; those on the tongue are the lingual teeth; and a transverse crescentic ridge, below the tongue, and produced into several cusps, is known as the mandibular tooth.

In P. marinus the two cusps of the maxillary tooth are close together, and the lingual teeth consist of two pairs of lunate denticulate ridges, the two ventral united with one another in the middle line. In P. fluviatilis the cusps of the maxillary tooth are somewhat widely separated, the dorsal lingual teeth are absent, and the ventral are united to form a strong transverse ridge, with a prominent median cusp.

25. The nostril, a single median aperture on the upper surface of the head, a short distance from its anterior end.

26. The eyes, situated at the sides of the head, a little posterior to the nostril; they are devoid of eyelids, but covered with transparent integument.

27. The external branchial apertures (Figs. 4 and 6 , e.a), a row of seven small slits on each side of the head, the first a little behind the eye.

28. The urinogenital papilla (Fig. 5, u.g.p), a small elevation, situated in the median ventral line, at about a quarter of the length of the body from the hinder end. It is pierced at its summit by a small opening-the urino. 
genital aperture $(z)$, - and lies in an oval depression, in the anterior part of which, just in front of the base of the papilla, is another small aperture, the anus $(a)$.

29. The two dorsal fins (Figs. 7 and 8 ), median longitudinal folds of the integument: the anterior end of the first is near the middle of the body, that of the second somewhat in front of a vertical line taken through the anus.

3o. The caudal fin, continuous in front with the second dorsal, and continued round the end of the tail on to its ventral edge; it is diphycercal, that is, evenly distributed above and below the axis of the tail.

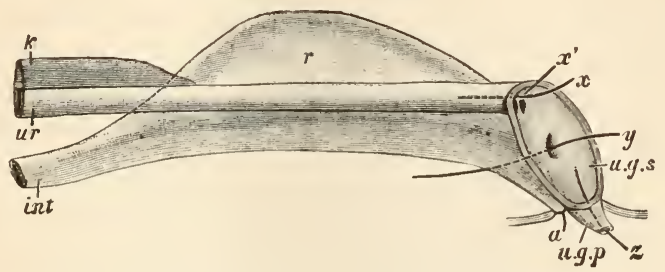

FIG. 5.-Petromyzon marinus. The urinogenital sinus, with the rectum and part of the left kidney (nat. size).

$a$, anus : int, intestine: $k$, left kidney: $r$, rectmm : $u . g . p$, urinogenital papilla: u.g.s, urinogenital sinus: ur, left ureter, $x$, aperture of left, and $x^{\prime}$ aperture of right ureter into urinogenital sinus : $y$, bristle passed through right abdominal pore : $z$, bristle passed into urinogenital sinus through its external aperture.

31. The absence of paired fins, or fore and hind limbs.

32. The apertures of the sensory tubes, minute punctiform openings on the surface of the head.

IV. Remove the skin from the anterior part of one side of the body, from the end of the mouth to a short distance behind the gill-slits, and from the middle dorsal to the middle ventral line: make out-

33. The body muscles, lying beneath the skin, and 
arranged in transverse segments, called myotomes or myocommas, separated from one another by strong plates of fibrous tissue.

Each myotome takes a zigzag course ; starting from the middle dorsal line, it passes first sharply backwards, then more gently forwards, then backwards, and finally sharply forwards. The plane of the myotomes is not at right angles to the long axis of the body, but is inclined from the surface, inwards and forwards. The muscular fibres of which they are composed are longitudinal, i.e. at right angles to the general direction of the myotomes themselves.

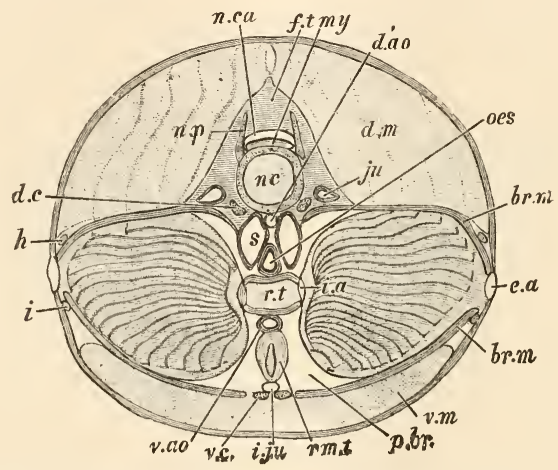

FIG. 6.-Petromyzon marinus. Transverse section through the branchial region, semi-diagrammatic (nat. size). The gill sacs are supposed to be rotated forwards, so as to bring the external in the same plane as the internal branchial apertures.

br.m., branchial membrane: d.ao, dorsal aorta : d.c, dorsal cartilage of branchial basket: $d . m$, dorsal body-muscles : e.a, external branchial aperture : f.t, fibrous tissue inclosing neural canal : $h, i$, lateral longitudinal cartilages of branchial basket : i.a, internal branchial aperture : $i . j u$, inferior jugular vein : $j u$, jugular vein : $m y$, myelon : $n c$, notochord : n.ca, neural canal : n.p, neural processes : ces, œsophagus : p.br, peri-branchial sinus : r.m.t, retractor muscle of tongue : r.t, respiratory tube : $s$, circum-œsophageal sinus : v.an, ventral aorta; z'.c, ventral cartilage of branchial basket : $v . m$, ventral body-muscle.

34. The division of the myotomes, immediately behind the last gill-cleft, into two longitudinal masses, a dorsal and 
a ventral, which pass forwards, the one above, the other below the line of branchial apertures, leaving these latter uncovered.

The dorsal muscle is inserted partly by a broad tendon to the fosterior dorsal cartilage ( $\S \mathrm{ro}$ ), and partly into the fibrous posterior wall of the orbit; the ventral muscle is inserted by a longish tendon into the annular cartilage ( $(16)$.

35. The great mass of radiating muscular fibre, which, now the skin is removed, forms the outer surface of the oral funnel.

V. Make a median ventral incision through the skin and muscle forming the walls of the body, beginning about the middle of the body, and proceeding forwards to within about half an inch of the last gill-cleft, and backwards to within about an eighth of an inch of the anus. From the ends of this incision carry up vertical incisions on the left side and remove the long flap thus made, so as to expose the body-cavity from the left side. Great care must be taken not to injure the liver (\$37), the left kidney $(\$ 40)$, or the rectum $(\$ 39)$. The following points can now be made out:-

36. The pigmented peritoneum lining the whole bodycavity.

37. The liver (Fig. 4, lr), at the anterior end of the cavity, deep red in the fresh condition in P. fluviatilis, greenish in $\mathbf{P}$. marinus. It consists of a single lobe, convex on the ventral side, excavated dorsally for the genital gland $(\$ 38)$ and intestine ( $\$ 39)$, hollowed out anteriorly for the pericardium $(\$ 44)$, which it partly covers, and ending in a blunt point posteriorly.

38. The single genital gland (testis or ovary), occupying in the adult the greater part of the body cavity: 
the testis is a soft, greatly lobulated organ, closely resembling fat: the ovary (Fig. $4,0 \%$ ), is also multilobular, each lobe containing numerous eggs, each about the size of a pin's head. The genital gland is suspended to the dorsal wall of the abdomen by a sheet of peritoneum, called mesorchium in the case of the male, mesoarium in that of the female. In the breeding season, the body cavity is found to be quite full of liberated ova, and the ovary itself is much reduced.

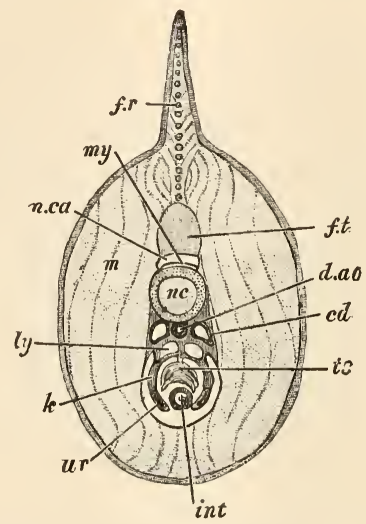

FIG. 7.-Petromyzon marinus. Transverse section through the abdominal region, including the first dorsal fin (nat, size).

$c d$, cardinal veins : d.ao, dorsal aorta : f.r, fin rays : f.t, fibrous tissue inclosing neural canal : int, intestine, the line pointing to the spiral valve $: k$, kidneys : $l y$, sub-vertebral lymph sinus $: m$, bodymuscles : $m y$, myelon : $n c$, notochord : $n . c a$, neural canal : $t s$, testis : ur, ureter.

39. The intestine (Figs. 4 and 7 , int), a straight, very slender tube, entering the body-cavity at its anterior end and on the dorsal side: it passes backwards between the liver and the genital gland, and then between the lobes of the latter, till it reaches the posterior end of the body-cavity, where it dilates into the rectum (Fig. 5, r), and ends by 
the anus $(a)$. The intestine is quite free for the greater part of its length, but at its hinder end is attached to the dorsal wall of the body-cavity by several delicate mesenteric bands, carrying blood-vessels, while in front it is firmly connected by fibre with the anterior half of the dorsal surface of the liver.

40. The kidneys (Figs. 5 and $7, k$ ), two elongated band-like organs, of a deep red colour, attached by peritoneum one on each side to the dorsal wall of the bodycavity, and extending from about the middle of the latter to within a short distance of the anus.

4I. The ureters (Figs. 5 and $7, u \%$ ), delicate tubes as wide as, or wider than the intestine, and attached all along the free (outer or ventral) edge of the kidneys: passing backwards they become connected with

42. The urinogenital sinus (Fig. 5, 1 .g.s), a small oroidal sac, lying close behind the rectum, and becoming narrowed ventrally to form the urinogenital papilla $(u \cdot g \cdot p)$.

43. The sub-vertebral lymph sinus (Fig. $7, l^{\prime}$ ), a considerable cavity included between the layers of peritoneum supporting the kidneys and genital gland : it is divided into two by a median vertical partition.

44. Note also the pericardium (Fig. 4, pc), lying just in front of and partly covered by the liver: its almost hemispherical posterior wall, strengthened by cartilage $(\S \mathbf{I} 8, \mathrm{Fig} . \mathbf{I}, p c)$ is all that can be seen at present.

VI. Open the urinogenital sinus hy carefully slitting up its left wall, first introducing a guarded bristle ${ }^{1}$ by the external aperture : observe-

45. The apertures of the ureters (Fig. 5, x, $x^{\prime}$ ), situated close together in the antero-dorsal region of the sinus.

1 That is, a bristle tipped with a small knob of sealing-wax. 
46. The abdominal pores (Fig. 5, y), small apertures situated one in each lateral wall of the sinus (the left will probably have been destroyed in opening the latter), and establishing a communication between the sinus and the body-cavity, through which the generative products escape.

VII. Open the intestine by a longitudinal incision along its whole length: note-

47. The spiral valve (Figs. 4 and 7 , int), a longitudinal fold of mucous membrane, projecting into the cavity of the intestine, and having a slight spiral twist.

48. The aperture of the œsophagus (Fig. 4, y), a longitudinal valve or slit, a short distance from the anterior end of the gut, and on its right side : in front of this aperture, the intestine is produced forwards into a short thick-walled, blind poucb, situated to the left of the posterior end of the gullet and of the sinus venosus (\$ 7 I). This will frobably be seen better at a later stage ( $\$ 59)$.

49. In P. marinus, the aperture of the bile duct (Fig. $4, b . d$ ), on the ventral wall of the gut, just at the point where the latter becomes free from its attachment to the liver. The bile duct is absent in the adult P. fluviatilis, and is not usually present in P. marinus, though very distinct in the specimen figured. The spiral valve begins just posterior to this point.

VIII. Turn the animal to the supine position (ventral aspect uppermost), and see the relations of the chief organs from the new point of view. Then remove the ovary or testis, the kidneys, and all but the anterior inch or so of the intestine: make out now-

50. The cardinal veins (Figs. 4,6 , and $7, c d$ ), two large vessels, lying one on each side of the middle line in the dorsal wall of the body-cavity.

51. The dorsal aorta (Figs. 4, 6, and 7, d.ao), a small vessel, lying in the middle line between the two cardinal veins.

52. Traced backwards, the cardinal veins are found at the posterior end of the body-cavity to unite into a single trunk, or rather to 
be formed by the bifurcation of a single trunk, the caudal vein (Fig. $8, c . v)$, which may be traced to the end of the tail, lying to the ventral side of the caudal artery (Fig. $8, c . a$ ), which is the direct continuation of the clorsal aorta.

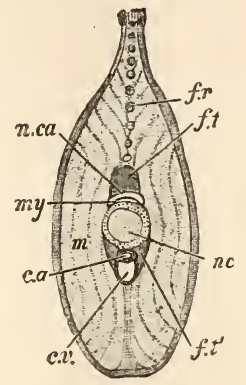

FIG. 8.-Petromyzon marinus. Transverse section through caudal region, including the second dorsal fin (nat. size).

$c . a$, caudal artery : $c . v$, caudal vein : f.r, fin rays : f.t, $f . t^{\prime}$, fibrous tissue enclosing neural canal : $m$, bcdy-muscles: $m y$, myelon: $n c$, notochord : $n . c a$, neural canal.

IX. Place the fish once more with the left side up- . wards ; dissect away the dorsal and ventral muscles of the branchial region ( $\$ 34)$, and, if the skeleton has not been examined, make out the various cartilages of the branchial basket ( $\$ \mathbf{r} 8)$ : then remove the latter, as well as the muscular and connective tissues obscuring the gill-sacs $\left(\$_{53}\right)$ : get the latter well cleaned, open one or two of them and note :-

53. The gill-sacs (Figs. 4 br, and 6), seven in number, arranged obliquely, each being inclined, from its inner side, backwards and outwards. They are compressed from before backwards, separated from one another by strong fibrous and muscular partitions, and marked externally with parallel horizontal lines, which mark the position of - 
54. The branchial filaments, sub-parallel ridges of the mucous membrane, lining the gill-sacs, and encroaching largely upon the cavity of the sacs.

The whole set of filamen's on each side of each sac constitutes a demibranch or half-gill : the septum between any two sacs, together with the two contiguous demibranchs, answers to a single gill of one of the higher fishes. (See p. $46, \S 84$, and p. 103, § 89.)

55. The internal branchial apertures (Figs. 4 and $6, i .(t)$, on the inner walls of the gill-sacs, leading into the respiratory tube presently to be seen $\left(\$ 5^{8}\right)$.

56. The peribranchial sinuses (Fig. 6, p.br), considerable spaces included between the gill-sacs themselves and the partitions which separate them.

$\mathrm{X}$. Remove the gill-sacs, cutting them away close round the internal branchial apertures, but taking great care not to injure the respiratory tube $(\$ 58)$, gullet (\$59), \&c.: also dissect away enough of the dorsal body muscles to allow of the structures mentioned being brought clearly into view. Observe the following:-

57. The retractor muscles of the tongue (Fig. 4, r.m.t), surrounding the lingual cartilage (\$ I4, Figs. I and $4, g)$, and forming a prominent cylindrical fleshy mass, in the middle ventral line, separating the gill-sacs of opposite sides, and extending back as far as the pericardium.

$5^{8}$. The delicate membranous respiratory tube (Figs. 4 and $6, r . t)$, lying almost immediately above the retractor of the tongue ${ }^{1}$ : the seven internal branchial apertures of the left side are seen perforating its lateral wall.

59. The œsophagus (Figs. 4 and 6, as), lying immediately above the respiratory tube, and becoming continuous

1 The ventral aorta which is between the two will be mentioned subsequently $(\S 72)$. 
posteriorly with the intestine in the antero-dorsal region of the body-cavity. Its diameter is not more than half that of the respiratory tube, and its walls are very delicate and easily torn. The best way to make it out without damage is to pass a guarded bristle into it from the already opened intestine.

6o. The pericardium, a large chamber, lying immed:ately behind the last pair of gill-sacs : its posterior wall, as already seen ( $\$ 44)$, is strongly convex; its anterior surface fits closely against the hinder or inner walls of the last pair of branchial sacs, and presents therefore a double concavity. In the present view, of course, only the left of these concavities is seen.

61. The circum-œsophageal sinus (Figs. 4 and $6, s$ ), a considerable longitudinal cavity, lying above and at the sides of the gullet, and imperfectly divided into two tubes by a perforated vertical partition which is related to the gullet like a mesentery. Behind the posterior end of the respiratory tube, the sinus extends also below the gullet as far downwards as the ventral aorta $(\$ 72)$ and between the inner or posterior walls of the last pair of gill-sacs, this portion (Fig. $4, s^{\prime}$ ) being also divided by a perforated vertical partition. The cavity is related to the gullet in much the same way as the body-cavity to the intestine.

XI. If the anterior (pre-branchial) portion of the ventral body-muscle ( $§ 34)$ is still left, remove it, and note

62. A great mass of muscle, forming a prominent projection beneath the eye, and just in front of the gills : it forms the lateral boundary of the mouth-cavity and contains imbedded in it the subocular arch $(\S 7)$, styliform process (§ 8$)$, and cornual cartilage (§ 9).

63. The salivary sac, appearing as a dark patch on the ventro. lateral region of the above mass of muscle : when cut into, it is seen to be a cavity with glandular walls : it is said to open by a fine duct into the mouth. 
XII. Remove the eye, and dissect away the muscles, cartilages, \&c. (\$ 62), which form the left side wall of the mouth-cavity, first passing a probe into the latter from the oral aperture to guard against cutting too deeply. Also open the œsophagus and the respiratory tube by a longitudinal incision along the left side of each. The following points can now be made out :-

64. The almost tubular oral cavity (Fig. 4, m), communicating with the oral funnel by the very narrow oral aperture.

65. The oral cæcum, a blind pouch of the mucous membrane in about the middle of the mouth-cavity, on the dorsal side (Fig 4, m); it is continued posteriorly into a longitudinal groove.

66. The aperture of the œsophagus (Fig. 4, between $m$ and $\alpha s)$, at the hinder end of the oral cavity, and immediately beneath it, that of the respiratory tube. Between the two apertures the mucous membrane forms a sort of horizontal shelf, produced into five finger-like processes in P. fluviatilis, and in P. marinus into two blunt processes supported by small cartilages. The entrance of the respiratory tube is also guarded by two lateral flaps of mucous membrane, the vela. (Fig. 4, vl).

67. The spirally arranged ridges into which the mucous membrane of the gullet is produced.

68. The extent of the respiratory tube, and the internal branchial apertures of the right side.

XIII. Open the pericardium by carefully removing the greater part of its left wall : note

69. The auricle (Fig. 4, au), occupying the whole of the left side of the pericardial chamber, and therefore the only part of the heart seen at first. It is strongly convex on its 
dorsal and left side where it comes in contact with the pericardium, while on the right it adapts itself to the form of the ventricle.

70. The ventricle (Fig. 4, $\tau^{\prime}$ ), seen by lifting up the auricle to lie towards the right side of the pericardium : it his a prismoidal form, and is of firmer texture than the auricle.

7I. The sinus venosus (Fig. 4, s.v), a tubular chamber, passing almost vertically from the dorsal to the ventral wall of the pericardium, between the auricle and ventricle: it is attached to the posterior wall of the pericardium by a thin vertical sheet of connective tissue.

72. 'The ventral aorta (Fig. 4. v.ao), springing from the anterior end of the ventricle, and passing through the wall of the pericardium, forwards and in the middle line, between the respiratory tube above and the retractor of the tongue below.

Opposite the fourth gill-sac the ventral aorta bifurcates and is continued forwards as two parallel trunks. From each of these are given off four afferent branchial arteries, the first supplying the first demibranch (anterior wall of first gill-sac), the second, the second and third demibranchs (posterior wall of first and anterior wall of second sac), the third, the fourth and fifth demibranch, and so on. The azygous posterior portion of the ventral aorta also gives off four afferent arteries, of which the first three supply each two contiguous demibranchs (i.e. one entire gill), and the fourth goes to the fourteenth or last demibranch. Each afferent artery is thus primarily related to a gill (\$ 54) and not to a gill-sac.

73. The inferior jugular vein (Fig. 4, i.ju) situated just below the retractor of the tongue and above the median ventral cartilage of the branchial basket. It brings the blood from the lower part of the head to the sinus venosus, and is best made out by making a small aperture in the latter and passing a probe forwards.

\section{The dorsal aorta (Figs. 4 \& 6, d.ao), immeciately}


beneath the notochord; it receives the efferent branchial arteries from the gills.

XIV. Remove the outer (left) wall of the auricle, and observe

75. The comparatively thin walls of the auricle, strengthened by a network of pectinate muscles.

76. The auriculo-ventricular valve (Fig. 4, see description), composed of two membranous flaps, and guarding the oval opening leading from the auricle to the ventricle.

77. The sinu-auricular valve (Fig. $4, x$ ), also composed of two flaps, and guarding the large aperture which leads from the sinus venosus into the auricle.

$\mathrm{XV}$. Pass a guarded bristle through the sinu-auricular valve upwards along the sinus venosus as far as it will go: also make an aperture in the left cardinal vein, pass a probe forwards, and open the vein along it.

78. It will be found that the cavities of the two cardinals unite with one another by an oval aperture (Fig. $4, x$ ) just above the dorsal end of the sinus venosus, and that, at the same place, they both communicate with the sinus.

79. The blo f from the anterior part of the body, except that returned by the inferior jugular, is brought back by the jugular veins (Figs. 4 and 6,ju), which lie one on either side of the notochord, and, uniting each with the corresponding cardinal, pour their blood into the sinus venosus. The jugular is best made out by passing a guarded bristle forwards from the cardinal and gradually dissecting down to it.

So. The hepatic vein (Fig. 4, h.v) may also be seen at this stage; leaving the liver, it pierces the pericardium and enters the sinus venosus at the same point as the inferior jugular.

XVI. Open the ventricle and note

8 I. 'The thick fleshy walls and small cavity. 
82. The two membranous semilunar aortic valves letween the ventricle and the ventral aorta.

XVII. Turn the fish with the dorsal side upwards; dissect away the muscles on the top of the head for about an inch ( $P$. fluviatilis) or two inches ( $P$. marinus) behind the nasal aperture; proceed until the brain, the nasal sac $(\$ \$ 9)$, and the auditory capsules $(\$ 5)$ are exposed; remove the roof from the latter. Observe the following :-

83. The flat band-like spinal cord or myelon (Figs. 4, $6-8, m y$, and 9), lying loosely in the neural canal (n.ca), and passing in front into the brain (Figs. $4 \mathrm{br}$, and 9), which nearly fills the small cranial cavity.

84. The medulla oblongata or myelencephalon (Fig. 9, m.o), the hindmost division of the brain, passing insensibly into the spinal cord behind, and on its upper surface presenting an escutcheon-shaped cavity, covered only by pia mater, the fourth ventric!e or myelocœle $(\pi \cdot 4)$.

85. The thin ledge of nervous matter orerhanging the anterior end of the fourth ventricle is all that represents the cerebellum or epencephalon (Fig. 9, $c b$ ).

86. The mid-brain, or mesencephalon (Fig. 9, o.l). consisting on the upper surface of a pair of rounded elevations, the optic lobes, immediately anterior to the medulla; they contain a cavity, the aqueduct of Sylvius, or mesocœle (aq.s), open above, save for pia mater, and in communication behind with the fourth rentricle.

87. The thalamencephalon, or twixt-brain (Fig. 9, th), situated just in front of the mesencephalon; it consists of two paired masses, the thalami optici, between which is a cavity, the third ventricle, or thalamocœle $\left(i^{\prime} \cdot 3\right)$, continuous behind with the aqueduct of Sylvius. 
88. Two paired, somewhat kidney-shaped masses, forming the anterior division of the brain, and having their convexities towards the middle line; they are divided by depressions on their outer sides into two parts, the smaller posterior part answering to cerebral hemispheres, or prosencephala (Fig. 9, c.h), the larger anterior part to olfactory lobes, or rhinencephala (olf).

S9. The nasal sac (Fig. 4, na), a rounded chamber, opening by a short tubular prolongation in the single nasal aperture. On opening, it is seen to be lined with strongly jigmented epithelium, and to have its walls much plaited.

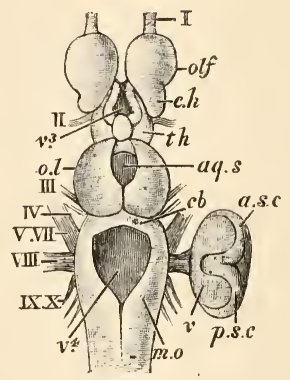

FIG. 9.-Petromyzon marinus. The brain from the dorsal aspect, with the right auditory $\operatorname{sac}(\times 3)$.

$a q . s$, aqueduct of Sylvius : a.s.c, anterior semicircular canal : $c b$, cerebellum : $c . h$, cerebral hemispheres : m.o, medulla oblongata : $0 . l$, optic lobes : olf, olfactory lubes; p.s.c, posterior semicircular canal : th, thalamencephalon: $v$, vestibule : $v \cdot 3$, third ventricle : $\tau^{\prime} \cdot 4$, fourth ventricle : $\mathrm{I}-\mathrm{X}$, cerebral nerves.

90. The olfactory nerves (Fig. 9, I), passing directly forwards from the rhinencephala to the nasal sac, through the apertures in the nasal capsule ( $(6)$.

9I. The optic nerves (Fig. 9, II), springing from the ventral surface of the thalamercephalon and passing directly outwards through the optic foramina ( $\$ 4)$ to the eyes.

92. The oculomotor (third) nerves (Fig. 9, III), springing from 
the un ler side of the mesencephalon, and passing outward; and slightly forwards to the orbit, where they supply most of the eye muscles.

93. The fourth pair of nerves (Fig. 9, IV), arising from the dorsal side of the anterior end of the medulla. They supply the superio: oblique muscles of the eyes.

94. The large nerve cord formed by the united roots of the fifth and seventh nerves (Fig. 9, V, VII), leaving the brain just in front of the auditory capsule, and passing forwards and outwards.

95. 'The auditory sac (Fig. 9, $\tau^{\prime}$ ), exposed by the removal of the dorsal wall of the auditory capsule; it consists of an ovoidal membranous sac, the vestibule ( $\left.i^{\prime}\right)$, presenting on its upper surface, towards the outer side, two semicircular canals (a.s.c, p.s.c), corresponding to the anterior and posterior canals of the higher vertebrata, the horizontal canal being absent.

96. The auditory nerve (Fig. 9, VIII), passing directly outwards from the medulla oblongata to the auditory sac.

97. The glossopharyngeal (ninth) and vagus (tenth) nerves (Fig. 9 , IX, X), arising from the medulla oblongata, just posterior to the auditory nerve, and passing backwards and outwards.

XVIII. With a guarded bristle find an aperture lying in the anterior ventral region of the nasal sac, close under the entrance of the external nasal canal (see Fig. 4) ; pass the bristle carefully into the opening; it will be found to take a course backwards and downwards. Without removing the bristle, turn the fish once more with its left side upwards, and dissect away the tissues between the anterior end of the notochord and the roof of the mouth, until the bristle is found. It will be found to have passed into

98. The nasal cæcum (Fig. 4, $n a^{\prime}$ ), a considerable pouch, lying close under the anterior end of the notochord. opening anteriorly; as already seen, into the ventral side of 
the nasal sac, and behind ending blindly a little posterior to the first internal gill-cleft.

XIX. If another specimen is available, make a series of transverse vertical sections through the entire animal previously hardened in alcohol or chromic acid (see Figs. 6-8). The sections should be about 2 to $3 \mathrm{~mm}$. thick in P. fluviatilis, 8 to ro $\mathrm{mm}$. in P. marinus. Be careful not to disturb the order of the sections. Examine each one separately, both from its anterior and its posterior face, and observe the precise relations of the various structures already seen by dissection. ${ }^{1}$

1 It is important not to omit this section unless time or material fails, as it forms an excellent introduction to the art of interpreting sections. 


\section{THE SKATE.}

The Skate (Raja batis).

The Thornback ( $R$. claíata).

The Homelyn Ray (R. maculata). ${ }^{1}$

\section{A.-THE SKELETON.}

I. 'THE skeleton of the skate is mainly cartilaginous, being hardened only by a superficial deposit of calcific matter, and in the vertebral column by internal laminæ of the same material. It is best prepared by immersing the fish, after removal of the viscera, for a few seconds in hot water a few degrees below the boiling-point. This process softens the muscles and ligaments, and allows of their being readily stripped off the cartilage without injury to the latter. When prepared in the cold, the toughness of the ligaments, especially in the region of the vertebral column, renders their removal a matter of great difficulty.

1 These are the three commonest species of the genus Raja brought to the London market. For the distinguishing characters of the three species see pp. $4 \mathrm{I}$ and $42, \S \S 54$ and 60 . Several of the figures are taken from the common New Zealand species, $R$. nasuta. 
As some parts of the skeleton are more delicate than others, it is advisable, after a very short immersion in hot water, to dissect off as much of the flesh as will readily come away, and then to remove the branchial arches ( $\$ 40$ and $4 \mathrm{I}$ ) and to disarticulate the skull and pectoral fins from their attachments to the vertebral column. The latter can then, if necessary, be subjected to a second immersion in hot water. In cleaning the skeleton special care is required to avoid injury to the branchial arches $(\S 4 \mathrm{I})$, the spiracular cartilage $(\S 37)$, and the labial cartilages ( $\$ 34)$.

The skeleton must not be allowed to dry, but should be either kept in spirit or prepared by the glycerine jelly process described on p. $2, \S$ I. In the latter case about three to five days' immersion in the glycerine fluid, and two to three days in glycerine jelly, is required. During the process of drying, it will be found advisable to fix out any parts which show a tendency to twist or warp with pieces of wood or strappings of paper or calico.

II. In the skeleton as a whole, note

I. Its division into $a$, vertebral column, including dorsal and caudal fins ( $\S 3-\mathrm{I} 6)$; $b$, skull, composed of the braincase (\$§ I 7-33), and of a number of loosely-attached cartilages, consisting chiefly of the jaws and of the gill-arches (\$§ 34-4I); c, shoulder-girdle and pectoral fins (\$§ 42-49); d, hip-girdle and pelvic fins (\$§ $50-53$ ).

2. The superficial crust of ossification on nearly all parts of the skeleton, except some of the smaller cartilages, which are entirely uncalcified, and the centra of the vertebræ, which are calcified in a special manner $(\S 5)$ : the 
ossifications are deposited in the form of minute polygonal plates, forming a mosaic over the whole surface.

III. In the vertebral column, make out

3. The anterior part of the column, consisting of a large mass of cartilage - the anterior vertebral plate (Fig. Io, a.v.p), - not divided into distinct vertebræ, except in its posterior ventral portion, where there are rudimentary centra. It presents an almost flat ventral surface, a strong median dorsal ridge, and two lateral ridges, which, at about the middle of the plate, are produced upwards, so as to be nearly as high as the median ridge. The neural canal, for the reception of the spinal cord, runs from end to end of the plate, the side walls of which are perforated by two rows of small apertures, for the passage of the dorsal and ventral roots respectively of the spinal nerves. The antero-inferior region of the vertebral plate is produced into a scoop-like odontoid process, which fits between the condyles of the skull ( $($ I9): on either side of this, on the front boundary of the lateral ridge, is a smooth surface, with which one of the condyles themselves articulates.

4. The remainder of the rertebral column, consisting of distinct vertebræ, which are divided into two sets: the trunk vertebræ, extending from the posterior end of the vertebral plate to the root of the tail, and the caudal vertebræ, reaching to the end of that appendage.

IV. Examine the trunk vertebræ; some from the surface; some by means of longitudinal and transverse sections; some by disarticulation from the rest; and others by allowing to dry. Make out

5. The centra of the vertebræ (Figs. II, I 2, and I3, c), cylindrical bodies with deeply-concave anterior and posterior ends; these ends are formed by concavo-convex plates of bone, which meet one another in the middle of the 


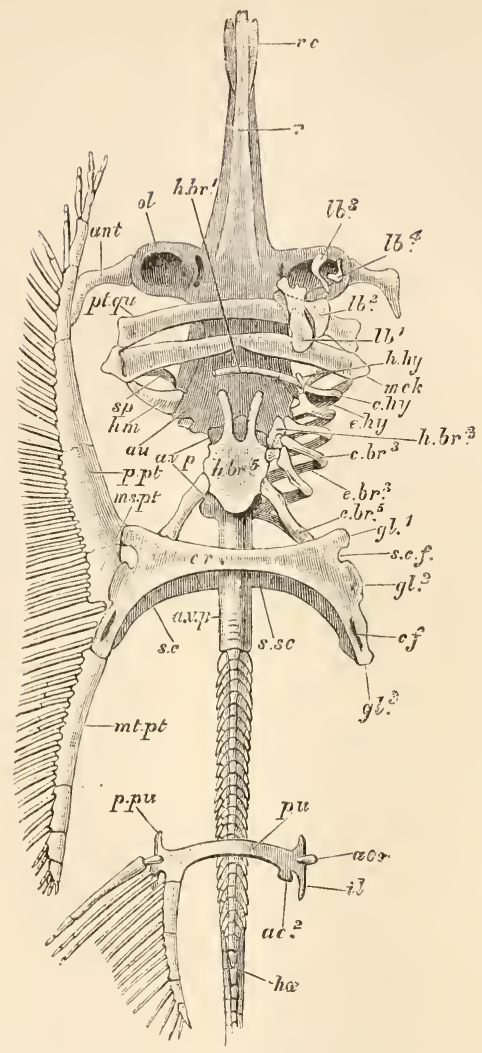

FIG. IO.-Raja nasuta (female). The skeleton seen from the ventral aspect (one fourth nat. size). On the left side (right in the figure) the pectoral and pelvic fins, and on the right the hyoid and branchial arches (with the exception of the fifth cerato-branchial) and labial cartilages are removed. In the remaining pectoral fin, only the proximal ends of most of the fin rays are shown, and the cartilaginous branchial rays are omitted for clearness sake.

ac. 1, ac. 2, anterior and posterior acetabular facets : ant, antorbital cartilage : $a u$, auditory capsule $: a . v \cdot p$, anterior vertebral plate $: c . b r .3$, $c . b r$. 5, third and fifth cerato-branchials : c.f, coracoid fontanelle $: c r$, 
coracoid : c./2y, ceratohyal : e.br. 3 , third epi-branchial : e./ry, epi-hyal : gl. I, gl. $2, g l .3$, anterior, middle, and posterior glenoid facets : h.br. I, h.br. 3, h.br. 5, hypo-branchials of the first, third, ancl fifth branchial arches: h.hy, hypo-hyal: $/ \mathrm{m}$, hyomandibular: he, commencement of hremal canal: il, iliac process: $l b .1, l 6.2, l b .3$, lb. 4, labial cartilages: mck, Meckel's cartilage or lower jaw : ms.pc, mesopterygium: mt.pt, metapterygium : ol, olfactory capsule : $p . p^{\prime}$, propterygium: $p \cdot p u$, pre-pubic process: $p t . q u$, pterygo-quadrate cartilage, or upper jaw : pu, pubic portion of hip girdle : $r$, rostrum : r.c, rostral cartilage : sc, scapula : s.c.f, scapulo coracoid fontanelle: $s p$, spiracular cartilage : s.sc, supra-scapula.

longitudinal axis of the centrum; the cartilage intervening between them is further strengthened by four radiating plates of bone, which give rise to a cruciform figure in a

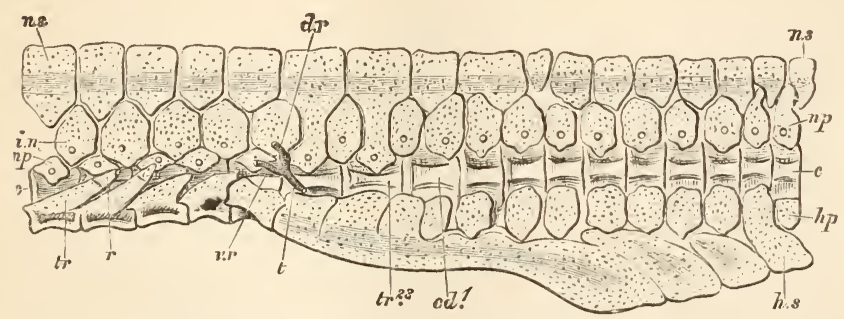

Fig. I I. - Raja nasuta. The last seven trunk vertebræ, and the first ten caudal vertebræ, viewed from the left side (nat. size).

$c$, centra: $c d$. I, first caudal veriebra : $d . r$, dorsal root of spinal nerve : h.p, hæmal process : h.s, hæmal spine : i.n, inter-neural plate : n.p, neural process: n.s, neural spine : $r$, rib: $t$, trunk of spinal nerve: $t r$, transverse process: $t r .28$, twenty-eighth (last) trunk vertebra: v.r, ventral root of spinal nerve.

transverse section of the centrum. The centra are seen to continue backwards the ventral portion of the anterior vertebral plate.

6. The transverse processes (Figs. II and I2, tr), backwardly directed rods of cartilage, which proceed one from either side of the centrum, and continue backwards the lateral ridges of the vertebral plate. 
7. The rudimentary ribs (Figs. I I and I2, $r$ ), small pieces of cartilage attached to the ends of the transverse processes.

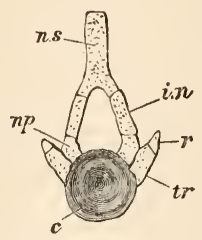

Fig. I2.-Raja nasuta. A trunk vertebra, seen from the front (nat. size).

$c$, centrum : $i . n$, inter-neural plate: $n \cdot p$, neural process : $n . s$, neural spine : $r$, rib : $t r$, transverse process.

8. The neural processes (Figs. I I, I 2 , and $\mathrm{I} 3, n . p$ ), small triangular plates rising one from each side of the dorsal surface of the centrum : a small aperture in each of them transmits the ventral root of a spinal nerve (Fig. I I, $z \cdot r)$.

9. The neural spines (Figs. I I, I2, and I3, n.s), flat plates dividing below into two triangular processes directed outwards, so that the whole plate has, in transverse action, the form of an inverted Y. Each neural spine is situated immediately above the vertebra of which it forms a part, but, save in exceptional cases, is not directly united with the neural processes, so that the neural arch is, so far, incomplete.

Io. The interneural (or intercrural) plates (Figs. I I, I2, and I3, i.n), six-sided plates of cartilage, which complete the neural arches laterally, filling up at the same time what would otherwise be the intervertebral foramina. Each transmits the dorsal root of a spinal nerve (Fig. II, $d . r)$, the ventral root of which traverses the neural process next in front. 
I I. The intervertebral substance, a gelatinous tissue filling up the biconvex spaces between contiguous vertebræ, and containing the remains of the embryonic notochord.

V. Examine the caudal vertebræ in the same way: make out the following points:-

I2. The centrum of each vertebra (Figs. I I and I $4, c$ ) gives off, on each side, a downwardly-directed hæmal process (h.p), which is seen, by examining the transition region between trunk and tail (Fig. I I), to be homologous with the transverse process of a trunk vertebra: the hæmal processes, uniting below, form the hæmal arch, which

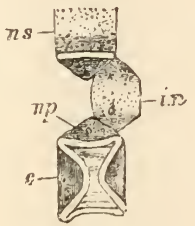

FIG. 13.-Raja nasuta. Longitudinal vertical section of a trunk vertebra (nat. size).

$c$, centrum: i.n., inter-neural plate: $n . f$, reural process: $n_{.} s$, neural spine.

transmits the caudal artery and vein (\$§ 157 and $x_{5} \delta$ ): a median process, the hæmal spine (h.s), is given off at the junction of the hæmal processes of most of the vertebræ, or may occur as a distinct cartilage (see Fig. I I).

I3. The absence of inter-neural plates and of ribs.

I4. The gradual reduction in size and simplification in structure of the vertebræ, seen in passing from the anterior to the posterior end of the caudal region.

r5. The skeleton of the two dorsal fins, attached to the posterior caudal vertebre: each consists of several radial cartilages or fin rays, attached to two basal cartilages, these latter being attached by fibrous tissue to 
several contiguous neural spines. Connected with the distal ends of the radial cartilages are numerous horny filaments, the dermal fin rays, which support the distal portion of the fins.

r6. The skeleton of the caudal fin is rudimentary, consisting of a few very small radial cartilages attached to the posterior caudal vertebræ: the fin is supported almost entirely by dermal fin rays.

VI. In the brain-case, note

17. The flat floor (basis cranii, Fig. 10) and roof (tegmen cranii).

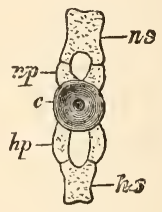

Fig, I4.-Raja nasuta. A caudal vertebra, seen from the front (nat. size).

$c$, centrum, h.p, hæmal process: h.s, hæmal spine: $n . p$, neural process : $n_{.} s$, neural spine.

r8. The foramen magnum, a large rounded aperture on its posterior wall, placing the cranial cavity in communication with the neural canal of the vertebral column. The cartilage surrounding the foramen magnum constitutes the occipital region of the skull.

19. The occipital condyles, rounded protuberances, one on either side of the foramen magnum, for articulation with the corresponding surfaces on the anterior vertebral plate: between them the base of the skull is scooped out for the reception of the odontoid process.

20. The auditory capsules (Fig. Io, au), large outstanding masses of cartilage, one on either side of the 
hinder or occipital region of the skull, and serving for the lodgment of the organ of hearing.

21 . In front of the auditory capsules-the interorbital region-the cranium narrows considerably, but soon widens out again to form

22. The nasal capsules (Fig. Io, ol), large outwardly projecting masses of cartilage, containing a cavity for the lodgment of the nasal sacs, which cavity is closed above, but widely open below, and communicates with the cranial cavity by a large aperture through which the olfactory nerve (§ I 79) passes (see $\S 32$ ).

23. In front of the nasal capsules the skull narrows again to form the long projecting rostrum (Fig. ro, $r$ ), at the sides of which the two thin rostral cartilages $(r . c)$ are attached.

24. In the postero-inferior region of the auditory capsule close to where it joins the occipital region, is an aperture leading by a canal excavated in the wall of the auditory capsule, into the cranial cavity, and serving for the transmission of the pneumogastric or vagus nerve ( $\left.\$ 2 \mathrm{I}_{4}\right)$.

25. A smaller aperture in the capsule external to the last: this gives exit to the glossopharyngeal nerve ( $\S 2$ I 5 ).

26. Two considerable apertures in front of the auditory capsule, where it joins the side wall of the skull: the upper and larger of the two is for the transmission of the fifth or trigeminal nerve, the smaller of the two, situated below and somewhat behind the first, for the seventh nerve or portio dura (§ I86).

27. On the upper surface of the auditory capsule, near the middle line, is an aperture-that of the aqueductus vestibuli-leading directly into the cavity of the capsule (see $\S 2$ I 3 ).

28. On the upper surface of the skull, between and in 
front of the auditory capsules, is a large oval space closed by dense fibrous tissue; this is the posterior fontanelle.

29. Anterior to and between the nasal capsules is a similar space, freely open in the prepared skull, the anterior fontanelle.

30. In the side-wall of the skull, about midway between the olfactory and auditory capsules, is the large optic foramen for the transmission of the optic nerve ( $\$$ I 83 ) : almost immediately above this are several very minute apertures through which the fourth nerve ( I $_{5}$ ) passes, while behind it is a small foramen for the passage of the third nerve (\$ I 84$)$.

3r. The nasal capsule is perforated by two canals just where it joins the skull, one through its upper part for the orbito-nasal nerve ( $\$ 205)$, another through its lower part for the palato-nasal nerve (\$209).

32. By making a longitudinal vertical section of the skull, or by removing its roof, the large olfactory foramen is seen, leading from the cranial cavity into that of the olfactory capsule, and giving exit to the olfactory nerve (\$ I 79).

33. In the same section of the skull is shown a large foramen-the internal auditory meatus-leading from the cranial cavity to that of the auditory capsule, and transmitting the auditory nerve $(\S \mathrm{I} 86)$ : a little posterior to this is a small aperture by which the glossopharyngeal nerve enters the auditory capsule to leave it by the foramen already seen $(\S 25$, see also $\S \S$ I 88 and 2 r 8$)$ : posterior to this again is the inner aperture of the canal for the vagus (§\$ 24, 1 89, 2 19).

VII. In the remaining loosely-attached portions of the skull make out

31. The labial cartilages (Fig. Io, $l h . \mathrm{I}-4$ ), of which 
there are altogether four, in connection with the ventral region of each olfactory capsule: the first (Ib. I) supports the corresponding flap of the fronto-nasal process (\$64), the second $(l b .2)$ lies in the fold of skin external to the nasobuccal groove $(\$ 64)$, the third $(l b .3)$ bounds the nostril internally, and the fourth ( $l b .4)$ externally.

35. The antorbital (palatine) cartilage (Fig. Io, ant), a thick rod, attached to the outer face of the olfactory capsule, and curving outwards and backwards to join the anterior prolongation of the pectoral fin.

36. The hyomandibular (Fig. Io, h.m), a club-shaped cartilage, articulating by its broad end with the inferoexternal region of the auditory capsule, and passing downwards and forwards.

37. The spiracular cartilage (meta-pterygoid) (Fig. ro, $s p$ ), a semi-lunar plate, attached by ligaments above to the auditory capsule and below to the inferior end of the hyomandibular.

38. The upper jaw (Fig. Io, pt.qu), formed by the fibrous union in the middle line of the two pterygoquadrate cartilages, which together form a strong, curved, transverse bar, connected at its extremities by ligaments to the hyomandibular and spiracular cartilages. Each end presents a smooth convex facet for articulation with the lower jaw.

39. The lower jaw or mandible (Fig. Io, mck), formed by the fibrous union in the middle line of the two Meckel's cartilages; it closely resembles the upper jaw in shape, and is articulated to it at its extremities. Both upper and lower jaws are more strongly calcificd than the rest of the skull.

40. 'The hyoid arch (Fig. Io, e.hy, c.hy, h.hy), a slender rod of cartilage, attached dorsally to the postero-superior 
angle of the hyomandibular by a band of fibrous tissue, the inter-hyal ligament, and made up of three segments, an epi-hyal (e.hy) above, cerato-hyal (c.hy) next, and hypo-hyal (h.hy) below.

4r. The five branchial arches (Fig. IO), each of which consists primarily of four segments, which are, counting from above downwards, pharyngo-branchial, epi-branchial $(e . b r)$, cerato-branchial (c.br), and hypo.branchial $(h . b r)$. The fourth and fifth pharyngo-branchials unite with one another and with the fifth epi-branchial to form a single cartilage: the first hypo-branchials (h.br.I) unite with one another in the middle line, forming a slender transverse bar of cartilage just posterior to the lower jaw : and the fifth hypo-branchials (h.br. 5) also unite with one another to form a large basi-branchial plate, produced anteriorly into two processes, and lying immediately dorsally to the heart and ventral aorta.

VIII. In the shoulder girdle, note

42. The complete union of the two halves of the girdle in the middle line below, and the attachment of each half above to the median dorsal ridge of the anterior vertebral plate (Fig. I0).

43. The three convex articular surfaces or glenoid facets (Fig. I , $g l$. I-3), situated on the external surface of each half of the shoulder girdle, and serving for the attachment of the three chief divisions of the fin (see $\S \S 46-48$ ). The part of the girdle which lies to the dorsal side of the glenoid facets is the scapular region (sc, s.sc), - that on the ventral side the coracoid region ( $c r$ ).

44. The three large apertures or fontanelles which perforate the shoulder girdle: the anterior of these is the scapulo-coracoid fontanelle (Fig. Io, s.c.f): the postero-superior, the scapular fontanelle, and the 
postero-inferior one, separated from the preceding by a bar of cartilage, the coracoid fontanelle $(c . f)$.

45. The separation from the remainder of the girdle of that part which is immediately attached to the rertebral column : this is the supra-scapula (Fig. Io, s.sc), a flat quadrate plate, attached by fibrous tissue to the scapula proper.

IX. In the pectoral fin, make out

46. The pro-pterygium (Fig. Io, p.pt), a long stout cartilage, articulated proximally to the anterior glenoid facet, and passing forwards and slightly outwards : it is continued forwards by a series of similar cartilages, of progressively diminishing size, which extend in front of the olfactory capsule, and one of which becomes connected with the antorbital cartilage.

47. The meso-pterygium (Fig. Io, ms.pt), a flat, comparatively small cartilage forming the central portion of the attached part of the fin: it articulates with the middle glenoid facet.

48. The meta-pterygium (Fig. Io, mt.pt), a similar cartilage to the pro-pterygium, which articulates with the posterior glenoid facet, and passes backwards and outwards : it is continued backwards by a series of smaller cartilages, which extend a little posterior to the pelvic girdle.

49. The fin rays, long jointed rods of cartilage, attached at their proximal ends to the three chief divisions of the fore-limb, and becoming very slender at their distal extremities : a few enter into direct connection with the shoulder girdle, between the meso- and meta- pterygium.

$\mathrm{X}$. In the pelvic girdle and fins, observe

50. The hip girdle, consisting of a stout ventral bar (Fig. Io, $p u$ ) which sends off on each side a forwardly directed pre-pubic process $(p \cdot p u)$ from the external 
extremity of its anterior edge, and an iliac process (il) directed upwards and backwards from the external extremity of its dorsal surface.

51. The acetabular facets (Fig. Io, ac. I, ac. 2), two projecting convex articular processes on the extremities of the hip girdle, the anterior being directed outwards, the posterior backwards.

52. The basale metapterygii, a stout backwardlydirected cartilage, articulating with the posterior acetabular facet: it is continued by smaller cartilages to the posterior point of the fin.

In the male this series of cartilages is directly continued into the skeleton of the claspers (see $\S 57$ ), which is composed of several more or less calcified cartilages, some of them produced into sharp processes of various forms.

53. The fin rays: the first four or five are attached to the pelvic girdle, pass almost directly outwards, and support the anterior lobe of the fin $\left(\xi_{5} 6\right)$; the first, or pre-axial ray, is many times thicker than any of the others, and articulates with the anterior acetabular facet: the remaining fin rays are connected with the basale metapterygii, pass outwards and backwards, and support the posterior lobe of the fin.

\section{B.-DIRECTIONS FOR DISSECTION.}

XI. Verify the following external characters :-

54. The extreme depression of the body from above downwards : its rhomboidal shape, the anterior angle of the rhomboid being formed by the snout, the lateral angles by the points of the pectoral fins, and the posterior angle by the root of the tail, which is slender, depressed like the body in its anterior part, but compressed from side to side posteriorly. 
In $R$. batis the angle of the extremity of the snout is acute, and the lateral or pectoral angle slightly so : in $R$. clavata and R. maculata the angle of the snout is obtuse and the pectoral angles nearly right angles.

55. The immense pectoral fins, of a triangular shape, the bases of the triangles extending from the anterior extremity of the snout to within a short distance of the base of the tail, and their apices forming the lateral angles of the rhomboidal body.

56. The pelvic fins, each of which lies in the angle between the posterior end of the corresponding pectoral fin and the root of the tail, and consists of two lobes, an anterior outwardly-directed narrow one, and a posterior broader one, produced backwards, parallel with the tail.

57. In the male, the claspers, elongated organs, each presenting a groove along the outer edge, and strengthened by a cartilaginous skeleton which projects into the cavity of the organ in the form of sharp plates of various shapes.

58. The dorsal fins, two small median lobes near the posterior end of the tail.

59. The caudal fin, a still smaller lobe, quite at the extremity of the tail.

6o. The characters of the integument: it is tough and strong; greyish brown on the dorsal, white or grey on the ventral side: in certain parts it is quite smooth and devoid of scales, but in others is roughened by minute asperities or by well-marked spines, both being the exposed portions of the placoid scales, each of which consists of a buttonlike bony base imbedded in the derm, and of a thorn-like enamelled portion, which projects through the epiderm on to the surface of the body: these scales are best made out by dissecting out one of the larger ones.

In $\mathbf{R}$. batis the dorsal integument is dark olive-green or brown, the ventral integument dark grey, with minute black dots marking the 
apertures of the sensory tubes: the only large spines are on the tail : the greater part of the skin is smooth.

In $R$. clavata, the skin is brown above, white below : the dorsal surface of the whole body is covered with asperities often produced into distinct spines : the latter are especially large near the eyes and in the middle line of the back and tail : in the female especially, large spines occur on both dorsal and ventral surfaces of the body.

In $R$. maculata the dorsal surface is brown with darker brown or black spots, the ventral surface white: the skin is nearly smooth, except on the tail and in the region of the eyes : in the male there is a patch of large movable spines on each side of the head and on each pectoral fin, both on the dorsal surface.

6r. The mouth, a wide, transverse aperture on the under surface of the head, supported by the strong upper and lower jaws, which can be felt through the skir.

62. The teeth, forming a close pavement of small enamelled plates, produced in some cases into sharp spines.

In $R$. clavata the teeth are pointed in the male, flat in the female.

63. The nostrils, moderate-sized apertures, one on either side of the under surface of the head, a little in front of the mouth.

64. The fronto-nasal process, an escutcheon-shaped fold of integument, extending from the posterior boundary of the nostrils backwards towards the mouth, at either angle of which it forms a rounded lobe, beset with fimbriæ. This lobe bounds internally, and converts into an incomplete canal, a groove-the naso-buccal groove-which extends from the nostril to the angle of the mouth.

65. The branchial apertures, five valvular slits on the ventral surface of the body, behind the mouth, those of opposite sides approaching one another posteriorly. ${ }^{1}$

${ }^{1}$ Behind the last pair of branchial apertures, on the skin covering the ventral portion of the shoulder girdle, is a pair of slit-like depressions, looking like an obliterated sixth pair of gill slits. 
66. The vent, or cloacal aperture, a large opening with tumid lips, near the root of the tail.

67. The abdominal pores (Figs. I 8 and I9, pp. 55 and 57 , a.p), small slits, one on each side of the middle line, just posterior to the vent.

68. A strong bar-the ventral portion of the shoulder girdle (Fig. Io, cr) - can be felt a little behind the last pair of gill slits, and a similar bar-the pelvic girdle $(p u)$ -a little in front of the cloaca. Between these lies the abdominal cavity, and in front of the shoulder girdle, between the branchial apertures, is the position of the pericardial cavity.

69. The eyes, on the dorsal surface of the head, just over the mouth: they are greatly depressed from above downwards, and have no eyelids.

70. The spiracles, two considerable apertures, one behind each eye : they communicate with the cavity of the mouth. The front wall of the spiracle is covered with fine ridges, which constitute the pseudobranchia, the remains of an embryonic gill. This is supported by a plate of cartilage which may be easily felt, called the spiracular cartilage ( $\$ 37$ ).

7 I. The sensory tubes, seen best on the ventral surface, as delicate, transparent, winding lines, arranged symmetrically on opposite sides of the body, just beneath the skin, on the surface of which they open by minute apertures.

XII. Make a median longitudinal incision, first through the skin, then through the underlying muscle, from the posterior edge of the shoulder girdle to the anterior edge of the hip girdle : make transverse incisions from both ends of this, and reflect the flaps of skin and muscle, so as to expose the abdominal cavity. Make out the following :- 
72. The peritoneum, a shining pigmented membrane lining the abdominal cavity and investing the viscera : from it is secreted the larger or smaller quantity of peritoneal fluid contained in the cavity.

73. 'The large, soft, trilobed liver, attached by a broad base at the anterior part of the abdominal cavity, to the hinder boundary of which its lobes extend; in colour it varies from light brown to dark olive green.

74. The stomach, largely concealed by the middle and left lobes of the liver, and passing towards the posterior side of the abdominal cavity, where it becomes narrowed and bent upon itself in a $\mathrm{U}$ shape.

75. The intestine, partly hidden by the right lobe of the liver; it is wider than the stomach and marked externally with a spiral groove.

76. 'The cloaca (Figs. I 8 and I $9, c l$ ), connected with the posterior end of the intestine and situated quite in the posterior part of the cavity: it is much larger in the female than in the male. To bring it clearly into view it is advisable to remove carefully the median portion of the pelvic girdle.

77. The spleen, a dark red lobulated body, situated between the limbs of the U-shaped stomach.

78. The pancreas, a firm whitish gland, consisting of two lobes, a large dorsal lobe, closely applied to the left side of the intestine and connected by a bridge of gland substance with the small ventral lobe, which lies to the ventral side of the junction between stomach and intestine.

79. In adult specimens, more or less of the testes (Fig. I8, $t$ ) (in the male) or of the ovaries (Fig. I9, ov) and oviducts $(o d)$ (in the female) will be seen without disturbing the other organs, at the sides of the abdominal cavity, on the dorsal side of (beneath in their present 
position) the foregoing viscera: they should be brought into view by turning over the latter.

8o. The kidneys (Figs. I 8 and $19, k$ ) are also seen when the other viscera are turned aside, as long, dark red bodies, lying one on either side of the middle dorsal line of the posterior end of the body cavity: they are often obscured by the thick pigmented peritoneum which covers them.

XIII. Make a median longitudinal incision from the anterior boundary of the shoulder girdle to about the level of the second gill cleft: take transverse incisions from both ends of this, and reflect the flaps made, so as to expose the pericardial cavity: note

SI. The strong fibrous partition bounding the pericardium posteriorly, and separating it from the abdominal cavity.

82. The heart (Figs. 17,20 , and $2 \mathrm{I}$ ), consisting of a fleshy ventricle $(v)$, from which is given off anteriorly, and somewhat to the right side, the fleshy tubular conus arteriosus $(c . a)$; and to the dorsal side of these (beneath in this position) the large thin-walled sub-triangular auricle (au), and posterior to this, the transverse, tubular, transparent sinus venosus $(s . v)$.

XIV. Remove the skin to a distance of about an inch on either side of the heart, so as to expose the gill-sacs : remove the ventral walls of the latter, so as to expose their cavities. Make out

83. The five pairs of gill cavities, separated from one another by complete partitions, and opening externally by the apertures already seen.

84. The red branchial filaments attached to both anterior and posterior walls of all the cavities except the 
last, the posterior face of which is devoid of them. Each set of filaments is a demibranch or half-gill: each partition with its pair of demibranchs constitutes a gill. It will be seen that there are four complete gills, and one odd half-gill forming the first of the series.

$\mathrm{XV}$. Make an incision through the sikin of the head, about an inch in front of the eyes, i.e. in the region of the anterior fontanelle: lifting up the edge of the incision, so as to see what you are about, cut away the roof of the skull and the overlying skin, until the brain and the anterior part of the spinal cord are exposed. If no other specimen is to be dissected, go over $\S \S \mathrm{I} 69-\mathrm{I} 89$; then remove the brain by cutting through the nerves and place it in strong methylated spirit. If a special dissection of the nervous system is to be made, remove to spirit at once, ${ }^{1}$ first noting

85. The dura mater, or membrane lining the cerebrospinal cavity, in which the brain and spinal cord lie.

86. The pia mater, a delicate membrane investing the brain and cord and containing numerous bloodvessels.

87. The arachnoid fluid contained in the cerebrospinal cavity.

88. A mass of gelatinous tissue filling up the anterior part of the cerebral cavity.

1 For the satisfactory dissection of the brain, it is necessary for it to be placed in spirit while fresh : it is therefore advisable for the student to reach this stage of the dissection by the end of the first day's work. For a permanent preparation, the brain should be placed for 24 hours in a saturated solution of zinc chloride, and then, after removal of the pia mater, into strong alcohol, which should be changed at least once (Giacomini's method): the brain, either separate or in situ, may then be prepared by the glycerine jelly process (p. $2, \S \mathrm{I})$. 
XVI. Place the animal with the ventral side upwards, turn the lobes of the liver forwards so as to expose the remaining abdominal viscera, and without cutting or tearing anything, make out the following points :-

89. The relations of the various parts of the alimentary canal: the œsophagus or gullet enters the abdominal cavity through its anterior wall, to the dorsal side of the liver, and almost immediately dilates slightly to form the stomach: this passes to the posterior boundary of the abdomen, becomes narrowed, turns upon itself, and passes forward to about the middle of the cavity, where it forms a thickening - the pylorus - and becomes continuous with the intestine: this is, in its anterior or proximal portion, devoid of the spiral groove mentioned in $\S 75$, and loses it again in its posterior or distal part, shortly before it passes into the dilated cloaca: the anterior smooth portion of the intestine may be called the duodenum, the middle, dilated, spirally-marked portion the colon, and the posterior smooth portion the rectum: to the latter is attached, dorsally, a small conical red body, the rectal gland.

90. The mode of attachment of the alimentary canal. The posterior part of the œsophagus and anterior part of the stomach are suspended to the dorsal wall of the abdominal cavity by a fold of peritoneum, the mesogaster: the rectum is similarly suspended by a second fold, the mesorectum. The greater part of the intestine, on the other hand, is entirely unsuspended, so that there is no mesentery proper.

91. The fold of peritoneum-gastro-hepatic omentum-which passes between the liver and stomach, and is joined near the latter by a similar but much longer fold, the 
duodeno-hepatic omentum-from the duodenum. In these folds are contained the various vessels passing to the liver ( $§ 93)$.

92. The attachment of the spleen to the dorsal wall of the stomach by a broad sheet of peritoneum, the gastro. splenic omentum.

93. The vessels ${ }^{1}$ contained in the duodeno-hepatic omentum, to see which the membrane should be put somewhat upon the stretch. These are the bile duct (Fig. I 5, c.b.a) to the animal's right side, the duodenal

${ }^{1}$ For the satisfactory dissection of the blood-vessels the fish should be injected : if not, they may be made out by inflating $\mathrm{with}$ air by means of an anatomical blowpipe. I find the following the most convenient method for injection. Have ready four of the movable cannulæ usually provided with injecting syringes, or if these are not at hand, four glass tubes drawn cut to the form shown in the annexed cut: the end $a$ is

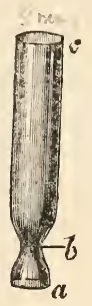
for insertion in the vessel, the constriction $b$ for the purpose of preventing any slipping of the ligature, over the end $c$ a short piece of india-rubber tubing is placed, and into this the nozzle of the syringe is pushed. Make a small incision into the conus arteriosus, place one cannula in it, directed forwards, and tie it firmly in its place: tie the second, directed outwards, into the sinus venosus : the third, directed forwards (i.e. towards the dorsal aorta), into the duodenal artery : the fourth, also directed forwards, into the duodenal vein. Fill an ordinary tumbler half full of fine plaster of Paris, coloured with a little of the common "French blue" cr ultramarine of the oil shops: fill up the tumbler with water, stir well and immediately strain the liquid through coarse muslin into a second tumbler. Fill the syringe, and inject through all four cannulæ successively. This must be done very rapidly or the plaster will set. On removing the syringe from a cannula, the india-rubber tube should be plugged with a small piece of wood to prevent ercape. All the chief vessels are injected in this way : the ventral aorta and its branches from the conus, the systemic veins from the sinus venosus, the dorsal aorta and its branches from the duodenal artery, and the portal vein form the duodenal vein. The caudal and renal portal veins have to be 
and superior mesenteric arteries (Fig. 20, $d, s . m$ ) towards the left, and the wide thin-walled portal vein (Fig. I6, $p$ ) between them.

Running parallel with these ressels is also a transparent thin-walled lymphatic vessel, from which many of the chief lymphatics can be injected.

94. The gall bladder (Fig. I 5, g.b), a greenish or yellowish transparent sac, partly imbedded in the liver between its right and middle lobes.

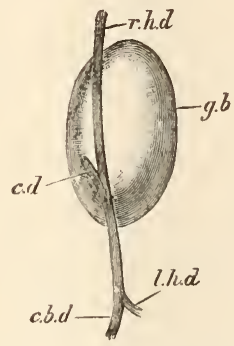

FIG. 15.-Raja nasuta. The gall bladder and bile ducts (half nat. size).

c.b.d, common bile duct: c.d, cystic duct: g.b, gall bladder: l.h.d, left hepatic duct : $r . h . d$, right hepatic duct.

XVII. Carefully dissect away the peritoneum from the bile duct: make a small aperture in its wall, introduce a blowpipe and inflate: the gall bladder will be distended : trace the duct in both directions and make out

done separately: the femoral and ilio-hæmorrhoidal veins also often escape being filled. In a preparation for demonstrating purposes it is advisable to colour the plaster of Paris used for injecting the dorsal aorta with vermilion or carmine instead of French blue. 
95. Its passage anteriorly into the gall bladder, by the short cystic duct (Fig. I 5, c.d).

96. Its entrance posteriorly into the duodenum, immediately behind the pylorus on the dorsal side. A bristle should be passed into the duodenum through the cut end of the duct.

97. The junction with it of the two hepatic ducts, one (Fig. 15, l.h.d) from the middle and left lobes, the other $(r . h . d)$ from the right lobe of the liver: the latter enterf the common bile duct close to its junction with the liver, so that the cystic duct $(c . d)$ is very short.

XVIII. Dissect away the peritoneum from the pancreas, and make out

98. The pancreatic duct, passing from the ventral lobe of the gland to open into the ventral wall of the duodenum, almost exactly opposite the entrance of the bile duct.

XIX. Dissect away the peritoneum from the blood vessels mentioned in $\$ 93$, and trace them in both directions, turning the stomach over to the left to see the origins of the arteries. Note

99. The portal vein (Fig. I6, $p$ ), passing towards the middle lobe of the liver, and sending off branches to all three lobes.

It is constituted distally by a gastric vein $(g)$, which receives the blocd from the stomach, and by a mesenteric vein $(d u, s p l, p n, i)$, which receives the blood from the intestine, spleen, and pancreas.

100. The cœliac artery (Fig. 20, co), entering the abdominal cavity on the dorsal side of the œsophagus and dividing into two trunks, one of which, the gastro-hepatic artery, sends off a hepatic artery $(h)$ to the liver and a gastric artery $(g)$ to the stomach, while the second or duodenal artery (d) passes down the duodeno-hepatic omentum, and supplies the greater part of the duodenum and the pylorus. 
I0I. The superior mesenteric artery (Fig. 20, s.m) entering the abdominal cavity parallel with, and a little posterior to the cœliac.

It divides into two main branches, one of which supplies the intestine (i), the other the pancreas $(p n)$ and spleen $(s p l)$. The rectum is mainly supplied by the small inferior mesenteric artery $(\$ 157$, Fig. $20, i . m$ ), which passes directly from the aorta to the rectal gland.

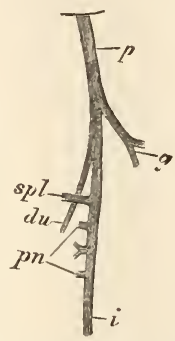

FIG. I6.-Raja nasuta. The portal vein (one-third nat. : ize). $d u$, duodenal vein $: g$, gastric vein $: i$, intestinal vein $: p$, main trunk of portal vein : $p n$, pancreatic veins: $s p l$, splenic vein.

102. The ramifications of the gastric branch of the pneumogasiric nerve on the walls of the stomach (see \$2I4).

XX. Turn the liver into its natural position again, and remove just sufficient of the shoulder girdle to bring into view

I03. The hepatic sinus (Fig. $7, h . s$ ), a large transverse ressel, lying across the ventral surface of the œsophagus, immediately in front of the anterior border of the liver: it receives the hepatic veins by which the blood is returned from the liver.

I04. The anterior extremities of the oviducts (Fig. I9, $\left.\delta^{t}\right)$ in the female, or the rudimentary pronephric ducts 
in the male (Fig. I8, pn.d, see $\S \S$ II $y$ and II 8.) The former are attached to the dorsal (under, in the present position,) surfaces of the shoulder girdle by a distinct ligament.

XXI. Remove the liver, taking care not to injure the hepatic sinus or oviducts: cut through the stomach just beyond its junction with the œsophagus : cut through the rectum just anteriorly to the rectal gland: remove the alimentary canal between these two points.

Wash out the contents of the alimentary canal by directing a stream of water through it, then fill with a 0.5 per cent. solution of chromic acid, by tying one end, pouring in the acid through a small funnel or injecting it with a syringe, and when full, tying the other extremity : place in a vessel of the same solution for a few days : when sufficiently hardened, cut windows in various parts and make out

105. The pyloric valve, a fold of mucous membrane extending between the stomach and intestine, and opening towards the latter.

106. The spiral valve, commencing in the duodenum as a simple inwardly-directed fold of the mucous membrane, and in the colon becoming a spiral inclined plane which finally terminates at the commencement of the rectum. The development of the spiral valve varies almost indefinitely: its width may be either less than, equal to, or greater than the semi-diameter of the gut : the plane of any part of it may be either at right angles to the long axis of the intestine, or inclined to it in either direction. There is also much variation in the number of turns of the spiral and in the character of the mucous membrane.

107. The irregularly longitudinal ridges or rugæ of the stomach.

I08. The network of fine ridges, covering the interior of the intesiine and the spiral valve.

XXII. If the fish is not injected, make a small aperture in the sinus venosus, introduce a blowpipe directed outwards, and inflate.

109. If nothing has been cut, a large inflated sac will be seen in the middle of the abdominal cavity, between the genital glands : this is the great cardinal sinus (Fig. I $7, c d . s$ ), formed by the union in the middle line of the two posterior cardinal veins $(c d)$, by which the blo 0 


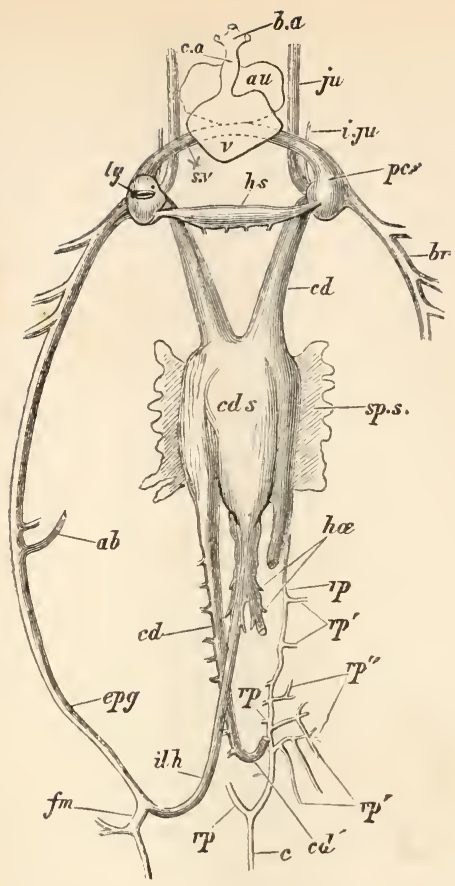

Fig. 17.-Raja nasuta. The venous system, ventral aspect (onethird nat. size).

The portal vein is not shown; the renal portal vein $(r . p)$ is supposed to be removed on the right side (left in the figure), and the femoral ( $f m$ ), ilio-hæmorrhoidal (il.h), \&c., veins as well as part of the cardinal $(c d)$ on the left. The right precaval sinus $(p c . s)$ is cut open, so as to show the apertures in its walls. The cutlines of those portions of the auricle $(a u t)$ and sinus venosus $(s . v)$ which lie behind (dorsal to) the ventricle $(v)$, are dotted.

$a b$, veins from abdominal walls : $a u$, auricle : $b . a$, bulbus anteriosus : $b r$, brachial vein : $c$, caudal vein : $c . a$, conus arteriosus : $c d$, cardinal vein : $c d . '$, posterior anastomosis of cardinal veins : $c d . s$, cardinal sinus : epg, epigastric vein : fm, femoral vein : h.s, hepatic sinus : ha, hæmorrhoidal veins: $i l . h$, ilio-hæmorrhoidal vein : $i . j u$, inferior jugular vein : $j u$, jugular vein : $l y$, opening of lymphatic trunk into precaval sinus : $p c . s$, precaval sinus: r.p, renal portal vein : $r . p^{\prime}$, factors of renal portal vein from pelvic and lumbar regions : $r \cdot p^{\prime \prime}$, branches of renal portal veins entering kidney: $s p . s$, spermatic sinus: $s . v$, sinus venosus : $v$, ventricle. 
is brought back from the tail, kidneys, genital crgans, cloaca, rectum, pelvic fins, \&c. These veins run along the inner side of the kidneys (see § I 30), and enter the sinus at about the anterior extremity of those glands: leaving the sinus, the cardinal veins are continued forwards and curve round the gullet to join the precaval sinus $(p c . s)$ : they are best seen at a future stage.

XXIII. The following organs are also best observed at this stage :-

I Iо. In the male, the testes (Fig. I 8, $t$ ), irregularly oval bodies, closely connected with the venous sinus, one on cither side. In the adult they have a flat ventral and a rounded dorsal surface: the former has a granular appearance, the latter is beset with snall rounded elevations. In the young condition the whole surface is quite soft and granular. At its anterior end, even in the adult, the testis is soft and thin, and passes into the upper end of the epididymis (see $\S$ II4) by which its secretion is carried off.

III. In the female the ovaries (Fig. 19, ov): these in the young condition are indistinguishable from testes, but in the adult state are covered with elevations varying from the size of the yolk of a hen's egg downwards-the Graafian follicles. Note the absence of any duct in direct connection with the ovary, the ova having to pass into the peritoneal cavity before reaching the aperture of the oviducts.

II 2. The fold of peritoneum called mesorchium in the male, mesoarium in the female, by which the genital glands are supported.

XXIV. Remove the genital glands and the venous sinus, and very carefully dissect away the peritoneum from the organs still left in the abdominal cavity, noting

113. The kidneys (Figs. 18 and $\mathrm{I} 9, k$ ), flat, reddish 


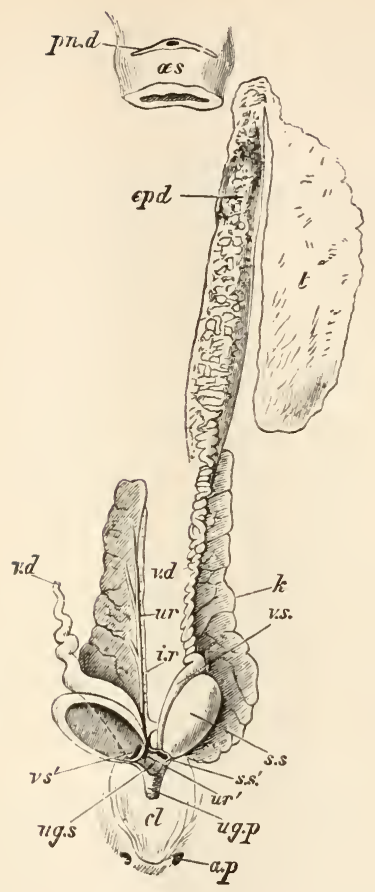

FIG. IS. - Raja batis. The urinogenital organs of the male (onethird nat. size).

The organs are supposed to be removed from the body along with a small portion of the osophagus ( $\propto s)$, and viewed from the ventral aspect : the right testis $(t)$ is removed, along with the corresponding epididymis $(e p d)$ and all but the posterior extremity of the vas deferens (v.d), which, with the sperm sac (s.s) is turned outwards to display the ureters $(u r)$ : the cloaca $(c l)$, the urinogenital sinus (ug.s), and the right vesicula seminalis $(v . s)$ and sperm sac $(s . s)$ are laid open.

$a . p$, abdominal pore : $c l$, cloaca : epd, epididymis : i.r, inter-renal body : $k$, kidney : as, œ.ophagus : pn.d, pronephric duct : s.s, sperm sac : s.s, its opening into the urinogenital sinus : $t$, testis : $u_{g} . p$, urinoge:aital papilla: $u g . s$, urinogenital sinus : $u r$, ureter: $u r^{\prime}$, its opening into the urinogenital sinus : $v . d$, vas deferens : $v \cdot s$, vesicula seminalis : $v \cdot s^{\prime}$, its opening into the sperm sac. 
brown, lobulated bodies, lying close to the dorsal wall of the abdominal cavity, and covered, on their ventral surfaces only, by peritoneum.

\section{In the male.}

I 14. The epididymes (Fig. I 8, epd), long flat, whitish bodies overlapping the anterior part of the kidneys, and continued forwards to the front boundary of the abdominal cavity. The greater part of the epididymis (mesonephros or parorchis) has a granular appearance, and its actual tubular structure is only seen in microscopical examination, but on its ventral surface it is very evidently made up of a greatly convoluted tube, which becomes more distinct and less convoluted near the posterior boundary of the parorchis, where it passes into

I 15. The vas deferens (Fig. I 8, v.d), mesonephric duct, or duct of the testis, a convoluted tube passing backwards from the hinder end of the epididymis, towards the posterior boundary of the abdominal cavity, where it becomes dilated.

I 16. The sperm sass (Fig. I8, s.s), large whitish ovoidal bodies, in the posterior region of the abdominal cavity, and immediately external to the posterior dilated portion of the vasa deferentia, from which they are separated externally by so slight a groove as not to be readily distinguishable from them. They are seen to advantage only in fully adult specimens.

II7. The coalesced remnants of the Müllerian or pronephric ducts (Fig. I8, pn.d), consisting of a fine thin-walled tube, situated on the ventral wall of the hepatic sinus, where it dies away at each side, and opens in the middle line by a small aperture homologous with the peritoneal opening of the oviducts in the female (see $§$ I I $\$$ ).

\section{In the fenale.}

I 18 . The oviducts (Fig. I9, fl.t, ut), each of which consists of two parts : a posterior, wide, thick-walled uterine 
portion (ut), opening into the cloaca, and an anterior, slender, thin-walled portion, or Fallopian tube ( $f$ t.t) which unites with its fellow of the opposite side on the

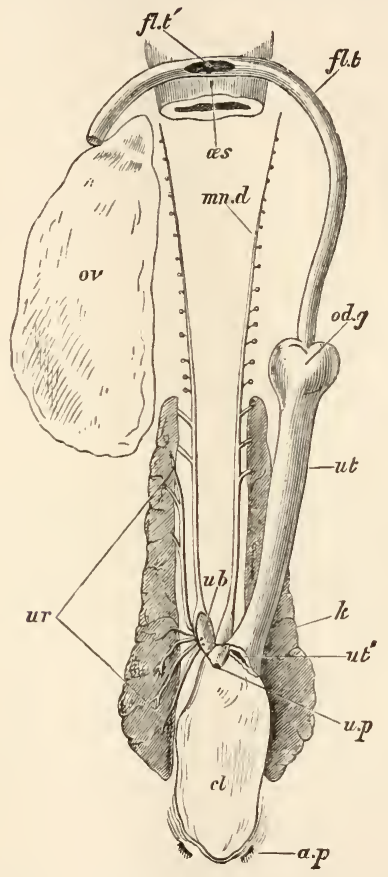

FIG. 19. - Raja batis. The urinogenital organs of the female (onethird nat. size).

The organs are removed from the body along with a small portion of the œsophagus (as), and are viewed from the ventral aspect. The left ovary $(o v)$ is removed as well as the greater part of the right oviduct; the cloaca and the right horn of the urinary bladder are laid open.

$a . p$, abdominal pore: $c l$, cloaca: $f$.t, Fallopian tube, or anterior portion of oviduct : $f$.t $t^{\prime}$, common opening of the conjoined Fallopian tubes into the abdominal cavity: $k$, kidney : $m n$.d, mesonephric duct : $o d . g$, oviducal gland : $\propto s, œ s o p h a g u s: o v$, ovary : $u . b$, urinary bladder : u.p, urinary papilla: $u$, ureters : $u t$, uterine portion of oviduct : $u t^{\prime}$, its opening into the cloaca. 
ventral surface of the gullet, where the two tubes have a common oval opening $\left(f l . t^{\prime}\right)$ into the peritoneal cavity. At the anterior end of the uterine portion of the oviduct is a large, solid, reniform body, of a whitish hue, imbedded in its walls: this is the oviducal gland $(o d . g)$, and secretes the horny "purse" in which the eggs are laid: it is not developed in very young specimens.

I19. The urinary bladder (Fig. I9, u.b) a bilobed sac with thin membranous walls, situated between the posterior ends of the oviducts : it will be better seen after distension with air (see $\$$ I 23 ).

$\mathrm{XXV}$. Open the cloaca by a median incision along its ventral wall, and note in it

I 20. The opening of the rectum.

In the male.

I 2 I. A small, thin-walled tube, the urinogenital papilla (Fig. I 8 , $u g . p$ ), projecting from the dorsal wall of the cloaca. By inserting a blowpipe into this and inflating, the sperm sacs and vasa deferentia will be dilated.

In the female.

I22. The large, thick-lipped apertures of the oviducts (Fig. 19, $u t^{\prime}$ ), one on each side of the anterior region of the cloaca. In young specimens each of these is closed by a fold of the mucous membrane, the hymen.

I23. A small conical elevation, the urinary papilla (Fig. I 9, $u . p$ ), situated between the oviducal apertures and having at its apex a small opening; by inserting a blowpipe into this and inflating, the urinary bladder will be distended.

In the male. ${ }^{1}$

XXVI. Slit up the urinogenital papilla, cutting along a probe or

${ }^{1}$ The following points ( $\$$ I24-127) can only be made out to advantage in fully adult specimens. 
blowpipe passed into it, and taking care not to go too far. The papilla will be found to lead into

124. The urinogenital sinus (Fig. IS, ug.s. ), a small cavity attached to the dorsal wall of the cloáca, into which it opens by the urinogenital papilla : at its anterior extremity will be seen two pairs of apertures, one pair $\left(s . s^{\prime}\right)$ ventrally situated, round, and opening into the sperm sac:, the other pair $\left(u r^{\prime}\right)$ dorsally situated with regard to the first, partly hidden by the projection into the sinus of the sperm sacs, of a somewhat crescentic form, and leading into the ureters (\$ I2S).

XXVII. Remove all that is left of the rectum, and of that part of the cloaca anterior to the urinogenital sinus : slit open the sperm sacs along their ventral wall; note

125. The greenish epithelium lining the sperm sacs, and the greenish viscid fluid which fills them, and which consists of semen mixed with the secretion of the sacs.

126. A crescentic, thick-lipped aperture (Fig. I $\left.8, v . s^{\prime}\right)$, on the dorsal wall of each sperm sac, just within (anterior to) the opening of the sac into the urinogenital sinus : this aperture leads into the vas deferens.

XXVIII. Open the lower part of the vas deferens and note

127. Its expansion posteriorly into a chamber-a sort of vesicula seminalis (Fig. 18, v.s), which has its walls produced into laminx, clividing its cavity into a number of compartments; at its posterior end this dilatation of the vas deferens opens into the sperm sac by the crescentic aperture already noticed.

XXIX. Carefully dissect one or both of the vasa deferentia from the surrounding parts, and either remove it entirely or turn it over to one side. Make out

12S. The ureter (metanephric duct, Fig. I8, ur), a delicate, colourless tube, situated close to the inner edge of each kidney; it is made up by lesser tubes proceeding from the several lobes of the kidney, and passes backwards to open into the urinogenital sinus by the slit-like aperture already noticed.

I 29. The inter-renal (Fig. I8, i.r), a long, irregular body of a yellow colour, lying just to the inner side of the ureter. 
The true adrenals or supra-renal bodies are small yellow masses in connection with the sympathetic ganglia.

I30. The cardinal veins (Fig. I 7, $c d$ ) lying to the ventral side of the ureters, receiving veins from the lidneys, uniting with one another posteriorly by a transverse anastomosis, and passing forwards to enter the cardinal sinus ( $\S 109$ ).

I31. The dorsal aorta (Fig. 20, d.ao), a delicate artery lying in the middle line between the kidneys, to which it sends branches. (See § I 57).

XXX. Dissect away the skin from the ventral face of one of the pelvic fins, and make out

132. The gland of the clasper, consisting of a large ovoiclal sac with thick muscular walls, which leads by a widish passage at its posterior end into the cavity of the clasper, and which receives the secretion of the gland itself. This is seen by cutting away the ventral wall of the sac to consist of two lobes, with a longitudinal groove between them, in which are the numerous papilliform terminations of the efferent ducts.

In the female.

XXXI. Turn over one of the oviducts and the corresponding half of the cloaca to the opposite side of the body, so as to expose one of the kidneys: dissect out-

I33. The ureters (Fig. I9 $u r^{\text {r }}$ ), delicate tubes proceeding from the inner edge of the kidney: those coming from the anterior part of the gland pass backwards, those from its posterior part forwards, towards the dorsal wall of the urinary bladder, which they enter.

134. A delicate thread proceeding forwards from each horn of the bilobed bladder, and presenting slight enlargements at intervals : this is the parovarium (Fig. I 9, mn.d) the rudiment of the anterior part of the Wolffian body or mesonephros of the embryo, and answering to the parorchis in the male.

XXXII. Remove the ventral wall of the urinary bladder, and note 
135. The three or four minute apertures on each side of the dorsal wall of the bladder, by which the ureters open.

136. The adrenals, inter-renals, dorsal aorta, and cardinal veins, which have the same relations as in the male (\$ई I 29I 3 I).

In both sexes.

XXXIII. Carefully dissect away the muscular tissue immediately anterior to the heart and between the two sets of gills, and make out

I37. The synangium or bulbus arteriosus (Figs. 20 and $2 \mathrm{I}, b . a$ ), a white dilatation, connected with the anterior cnd of the conus arteriosus or pylangium (c.a, $\$ 82)$, and giving off three vessels, one anteriorly, the ventral aorta (v.ao), and one on each side, the posterior innominate arteries $(p . i n)$. The ventral aorta passes forwards to the level of the anterior gill cavity, and there divides into two vessels, the anterior innominate arteries $(a . i n)$ which are given off at right angles to the ventral aorta, one on either side. Each anterior innominate artery soon divides into two trunks, each posterior innominate into three; these five vessels are

138. The afferent branchial vessels (Figs. 20 and 21 , a.br. I-5), each of which goes to one of the plates by which the gill cavities are separated from one another, passes from the ventral to the dorsal extremity of the plate, external to the branchial arch (see $\$ 166$ ), and gives off branches to the gill filaments.

139. The hypobranchiai artery (Figs. 20 and 21, hy.br), running antero-posteriorly, just internal to the gills, and crossing the afferent branchial arteries : it springs from the brachial artery (\$ 1 57), anastomoses with the efferent branchial arteries (\$ I56), and, besides supplying the tissue of the gills, sends off an anterior coronary artery (Figs. 20 and $2 \mathrm{I}, a . c 0)$ to the conus arteriosus and ventricle, and a posterior coronary $(p . c o)$, to the sinus venosus. 


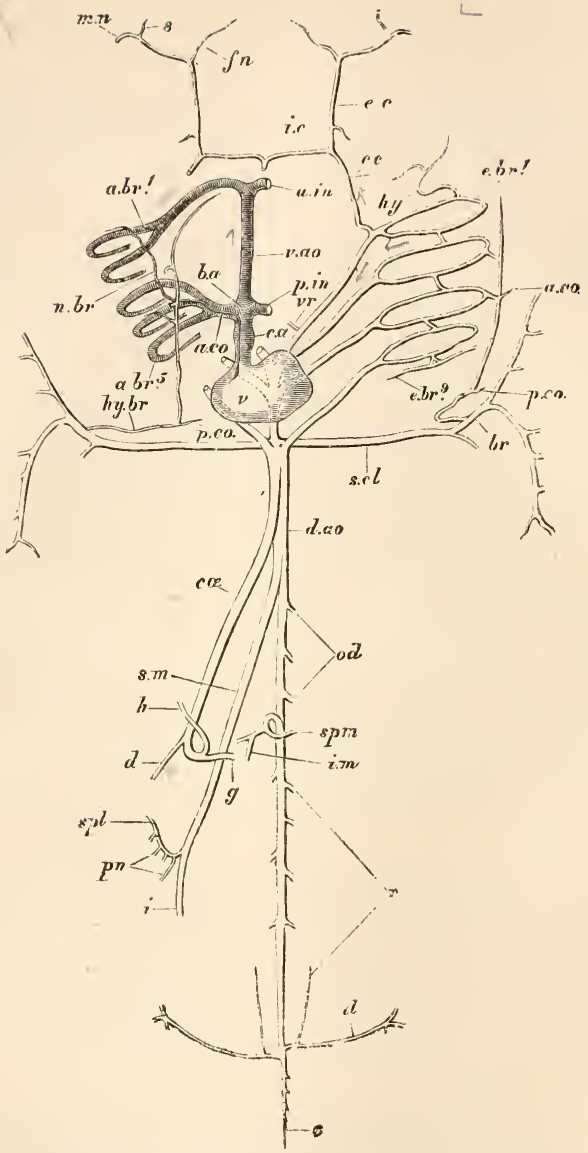

FIG. 20.-Raja nasuta. The arterial system, seen from the rentral as pect (one-third nat. size).

The heart and ventral aorta $(v \cdot a o)$ are slightly displaced towards the right side (left in the figure): the left afferent branchial arteries $(a . b r)$ are removed, and the right efferent branchials $(e . b r)$ : the lett efferent branchials are straightened out, so as to bring their ventral ends to the 
extreme right of the figure: the left hypobranchial artery is also displaced outwards; that of the right side (left in the figure) (hy,br) is drawn in situ.

$a . b r .1$, first, and $a . b r .5$, last afferent branchial artery : a.co, anterior coronary artery : $a . i n$, anterior innominate artery: $b . a$, bulbus arteriosus : $b r$, brachial artery: $c$, caudal artery : c.a, conus arteriosus: c.c, common carotid artery : $c \infty$, cœliac artery : $d$, duodenal artery : d.ao, dorsal aorta : e.br. 1, first, and e.br. 9, last efferent branchial artery : e.c, external carotid artery : $f . n$, artery to fronto-nasal process : $s$, gastric artery: $h$, hepatic artery : hy, hyoidean artery : hy.br, hypobranchial artery : $i$, intestinal artery : i.c, internal carotid artery : $i l$, iliac artery : $i . m$, inferior mesenteric artery : $m n$, artery to mandible : $n . b r$, nutrient arteries of the gills : od, oviducal arteries : $p . c o$, posterior coronary artery: p.in, posterior innominate artery: pn, pancreatic arteries: $r$, renal arteries: $s$, artery to snout: s.cl, subclavian artery: s.m, superior mesenteric artery: $s p l$, splenic artery : $s p m$, spermatic artery : $v$, ventricle $: v . a o$, ventral aorta $: v r$, vertebral artery.

140. The inferior jugular vein (Figs. 17 and $2 \mathrm{I}, i . j u$ ) situated in the dorsal wall of the pericardium, near its outer border : it brings blood from the floor of the mouth and pericardial walls, and enters the precaval sinus (\$ I49, Figs. I 7 and 2 I, pc.s).

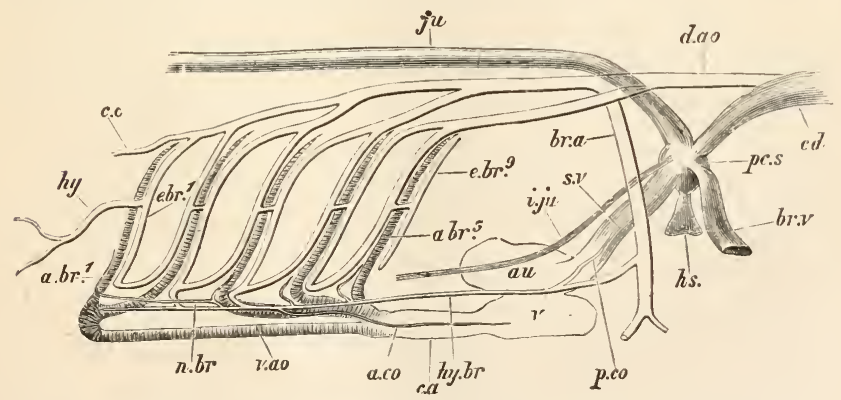

FIG. 2I.-Raja nasuta. Diagram of the heart ard chief bloodvessel :, from the left side (half nat. size).

$a . b r$. I, first, and $a . b r .5$, last afferent branchial artery : a.co, anterio: coronary artery: $a u$, auricle : br.a, brachial or subclavian artery: $b r . v$, brachial vein : $c . a$, conus arteriosus : $c d$, cardinal vein : $d . a c$, dorsal aorta : e.br. I, first, and e.br. 9, last efferent branchial artery : $h . s$, hepatic sinus: hy, hyoidean artery: hy.br, hypo-branchial artery : $i . j u$, inferior jugular vein $: j u$, jugular vein : $n . b r$, nutrient arteries cf the gills: $p . c o$, posterior coronary artery : pc.s, precaval sinus : s. $\tau^{\prime}$, sinus venosus : $\tau^{\prime}$, ventricle : $v_{\text {. }} u$, ventral aorta. 
I4I. The thyroid gland, a flattened rounded body, of a deep red colour, situated immediately in front of the anterior end of the ventral aorta.

XXXIV. Cut through the ventral aorta and posterior innominate arteries close to the bulbus arteriosus, and turn the heart backwards, so as to expose the pericardial cavity. Note

142. An aperture in the centre of the posterior dorsal region of the pericardium : by passing a probe into this it will be found to lead into a funnel-shaped cavity which soon divides into two membranous canals. These-the pericardio-peritoneal canals-pass backwards along the ventral wall of the gullet, and open each by a widish aperture, thus placing the pericardium in communication with the peritoneal cavity.

XXXV. Remove the heart entirely, by cutting through both ends of the sinus venosus and the membrane by which it is united to the pericardium: make out carefully the relations of the various parts of the heart $\left(\S_{2}\right)$, then cut open, first the auricle and sinus venosus from the dorsal side, and afterwards the ventricle and conus arteriosus from the ventral side. Make out

I 43. The thin, smooth walls of the sinus venosus.

I44. The thin walls of the auricle, strengthened by a complicated network of muscular fibres, the musculi pectinati.

145. The large sinu-auricular aperture guarded by the two membranous flaps of the sinu-auricular valve.

I 46. The circular auriculo-ventricular aperture, guarded by the two long flaps of the auriculo-ventricular valve, which are attached round the margins of the aperture, and hang down into the ventricle.

I 47. The small, horseshoe-shaped cavity of the ventricle, and its immensely thick walls, strengthened internally by muscular ridges or columnæ carneæ.

148. The three longitudinal rows of aortic valves in 
the conus arteriosus, each row containing five somewhat semilunar flaps, opening towards the synangium.

XXXVI. Pass a probe, directed outwards and backwards, into either of the cut ends of the sinus venosus : carefully cut away the cartilage of the shoulder girdle and other tissues until the end of the probe is brought into view : it will be found to have passed into

149. A small chamber, the precaval sinus (Figs. 17 and $21, p c . s$ ), situated in the antero-lateral angle of the abdominal cavity. In its wall are several apertures : one, situated antero-internally, is the opening of the jugular vein (iu), by which the blood is returned from the head : another, postero-internal in position, is the opening of the cardinal vein $(c d)$ already seen : a third, on the ventral wall of the sinus, puts it in communication with the hepatic sinus $(h . s):$ a fourth, at its anterior end, leads into the sinus venosus $(s . v)$ : a fifth, very small, just internal to the last, into the inferior jugular vein $(i, j u):$ a sixth, on the outer wall, into the brachial vein (br) and lastly, in the middle of the dorsal wall, is a transverse aperture, guarded by two valves, by which the contents of the chief lymphatic trunk (Fig. I 7 , ly) are poured into the sinus.

150. A sympathetic ganglion (see $\$ 220$ ), a whitish elongated body about a quarter of an inch long, will be found immediately outside the inner wall of the sinus.

XXXVII. Cut away the remainder of the ventral portion of the shoulder girdle, and cut through the floor of the mouth, by making an incision a little to one side of the median line so as not to injure the ventral aorta, through the œsophagus and basibranchial plates (see $\S 4 \mathrm{I}$ ), and through both jaws. Fasten back the two halves of the mouth-floor thus separated, so as to expose the whole oral cavity from beneath. Note

I 5 I. The internal branchial clefts, five long vertical fissures, by which the gill pouches communicate with the cavity of the mouth.

I 52. The opening of the spiracle into the mouth, just in front of the first gill cleft. 
I53. 'The curved hard bars which separate the gill clefts from one another: these are the branchial arches.

I 54. A similar hard bar of cartilage separating the spiracle from the first gill cleft: this is the hyoid arch.

I 55. The relations of the branchial filaments to the branchial arches : there is a set of filaments on both the anterior and posterior walls of all the gill cavities except the last, the posterior wall of which is devoid of filaments. Thus there are nine sets of filaments or half-gills in all, one of which occurs on the posterior face of the hyoid arch, and one on each face of each of the first four branchial arches, the fifth branchial arch being without filaments.

XXXVIII. Carefully dissect away the mucous membrane from the roof of the mouth; remove the dorsal ends of the gill arches or pharyngo-branchials; and follow out

I56. The efferent branchial arteries (Figs. 20 and $2 \mathrm{I}$, e.br. I-9), of which there are nine, one for each demibranch : after leaving the dorsal ends of the gills they unite with one another in pairs, that from the hyoidean demibranch uniting with that from the anterior demibranch of the first branchial arch, that from the posterior demibranch of the first with that from the anterior demibranch of the second branchial arch, and so on. Four trunks are thus formed, the last of which receives the vessel from the posterior demibranch of the fourth branchial arch, and the first two of which soon unite with one another, so that there are now three main efferent branchial trunks on each side. These take a direction inwards and backwards. The most anterior of the three pairs unite with one another in the middle line and form a short trunk: with this the next pair unite and produce a somewhat larger trunk, with which, finally, the third pair join. The longitudinal vessel thus 
formed by the union of the efferent branchial trunks is the dorsal aorta (§ I 57 ).

The efferent branchial arteries unite with one another in the way described at their ventral as well as at their dorsal ends, thus forming complete loops, the spaces enclosed by which correspond to the branchial clefts. The two arteries of each complete gill are united with one another by a cross branch at about the middle of their length : it is through this anastomotic branch of the last gill that the ninth efferent artery (e.br. 9) pours its blood into the eighth. From the junction of the third and fourth and of the fifth and sixth arteries, short anastomotic branches go off to the hypobranchial artery (\$ I39).

I57. The dorsal aorta (Figs. 20 and $2 \mathrm{I}$, d.ao), passing along the ventral face of the vertebral column : it gives off, just before being joined by the third pair of efferent branchial trunks, a large vessel from each side, which passes directly outwards to the pectoral fin: this is the brachial or subclavian artery (Fig, 20, s.cl, Fig. 2I, br.a). The aorta then passes backwards in close contact with the vertebral column to the posterior extremity of the abdominal cavity, giving off at about the level of the shoulder girdle two large vessels, the cœliac (Fig. 20, co), and superior mesenteric $(s . m)$ arteries ( $\$$ IOO and IOI), then the arteries to the oviducts $(c d)$ or epididymes, spermatic arteries $(s p m)$ to the testes or ovaries, a single inferior mesenteric artery $(i . m)$, ( $\$$ IOI), the numerous small renal arteries $(r)$ to the kidneys, and vessels to the pelvic fins and the parietes of the body. At the end of the abdominal cavity the dorsal aorta becomes the caudal artery $(c)$, which passes through the hæmal arches of the caudal vertebræ (Fig. Io, he) to the end of the tail.

158. The caudal vein (Fig. I7, c), running parallel and ventral to the caudal artery : on leaving the hæmal canal it divides into the two renal portal veins $(r p)$, which pass to and ramify in the corresponding kidneys $\left(r p^{\prime \prime}\right)$ receiving numerous veins $\left(r p^{\prime}\right)$ from the pelvic and lumbar regions. 
159. The femoral veins (Fig. 17, $\mathrm{fm}$ ) bringing the blood from pelvic fins : each divides into two trunks : one, the ilio-hæmorrhoidal vein $(i l . h)$, passes at first backwards and inwards, then forwards along the lateral surface of the rectum and cloaca, and opening finally into a posterior prolongation of the cardinal sinus : the second of the two veins into which the femoral divides is the epigastric vein $(e p g)$; it passes almost directly forward along the lateral wall of the abdominal cavity, receiving veins from the abdominal walls $(a b)$, and finally unites with the brachial vein $(b r)$.

It will be seen from Fig. I7, that the brachial, epigastric and iliohæmorrhoidal veins really form one continuous trunk, opening anteriorly into the precaval, posteriorly into the cardinal sinus, and receiving veins from the pectoral and pelvic fins, the abdominal walls, and the rectum and cloaca. The anterior portion of the epigastric should probably be considered as representing the mammary vein of mammals, the two being continuous instead of merely anastomosing.

160. The common carotid artery (Fig. 20, c.c), a small vessel springing from the efferent branchial vessel of the hyoid arch : it first passes somewhat inwards, and then directly forwards near the outer edge of the base of the skull, sending a branch inwards-the internal carotid artery (i.c) - which unites with its fellow of the opposite sicle to form an azygos trunk which perforates the base of the skull and is distributed to the brain, especially to the pituitary body and saccus vasculosus ( $\$$ I 76 and 177 ) : the external carotid (e.c) then passes forwards and slightly outwards, and sends branches to the jaws, snout, \&c.

161. The vertebral artery (Fig. 20, vr), a small trunk arising from the first of the three efferent branchial trunks : it passes inwards and slightly forwards, perforates the base of the anterior vertebral plate ( $\S$ ), and is distributed to the brain and spinal cord, on the ventral surface of which it forms, with its fellow, a plexus.

162. A small hyoidean artery (Fig. 20, hy) given off from the first efferent branchial : it passes to the dorsal side of the hyomandibular and sends a branch to the pseudobranchia.

XXXIX. Remove two of the gills and make out, in one by dissection, in the other by transverse sections

163. The cartilaginous branchial arch which supports the inner edge of the gill. 
164. The fibrous partition continued from the outer face of the branchial arch, and supported by the cartilaginous branchial rays.

I65. The soft vascular branchial filaments covering both sides of the partition, to which they are firmly connected along almost their whole length, their outer-extremities only being free.

166. The afferent branchial artery, running along the outer face of the branchial arch, in the middle line: it is of considerable size at the ventral end of the gill, but gradually diminishes towards its dorsal end.

167. The efferent branchial arteries, two trunks smaller than the afferent vessel, and running along the lateral edges of the arch, at the bases of the rows of filaments. They are of considerable size at the dorsal end of the arch, and diminish sumewhat towards its ventral end.

168. The thymus glands are exposed by the removal of the gills: each is a whitish body, about an inch long, lying immediately dorsal to the pharyngo-branchials.

\section{C.-SPECIAL DISSECTION OF THE NERVOUS SYSTEM AND SENSE ORGANS.}

$\mathrm{XL}$. It is advisable to take a fresh skate for the nervous system, at least unless the subject used for the foregoing work has been well preserved in strong spirit. The viscera, with the exception of the kidneys and vasa deferentia, may be removed at once.

Expose the brain and anterior part of the spinal cord, as directed in $\S \mathrm{XV}$., p. 46 . If a preserved brain is not available, the verification of the description of the ventral surface must be left until the origins of the nerves have been made out ( $\$ \$$ I $79-$ r 89 ) when the brain may be removed. Observe the following:- 
I69. The spinal cord (Fig. 22, my) lying in the neural canal of the vertebral column, and, at the junction of the latter with the skull, passing insensibly into the brain. It is divided into a pair of dorsal and a pair of ventral columns by deep dorsal and ventral fissures, and is traversed through its centre by the minute central canal.

I 70. The medulla oblongata or myelencephalon (Figs. $22-25, m .0$ ), the hindmost division of the brain : passing forwards from its junction with the spinal cord, it undergoes a gradual increase in diameter, and is produced dorsally, on each side, into a greatly convoluted body, the corpus restiforme (Fig. 22, c.r). Both dorsal and ventral surfaces of the medulla are marked with obscure median grooves, continuous respectively with the dorsal and ventral fissures of the spinal cord : the dorsal groove separates the dorsal pyramids, the ventral groove the ventral pyramids of the medulla.

I 7 I. The cerebellum (Figs. 22, 24, 25, cb), a median structure, situated to the dorsal side of the myelencephalon, and composed of two distinct lobes, a posterior, having the form of an isosceles triangle with the apex directed backwards, situated between the restiform bodies, and a squarish anterior, partly overlapping the optic lobes (§ I 73). Both lobes are marked on the surface by ridges (gyri) with interrening depressions (sulci).

I72. A small transparent area behind the cerebellum (Fig. 22, $z^{\prime} .4$ ), roofing over the fourth ventricle ( $\$$ r 80).

I 73. The mesencephalon (Figs. $22-25$, o.l, c.c) consisting on the upper surface of the two optic lobes (o.l), ovoidal bodies, lying just in front of, and partly overlapped by the cerebellum. The under surface of the mesencephalon is formed by the crura cerebri (c.c), 


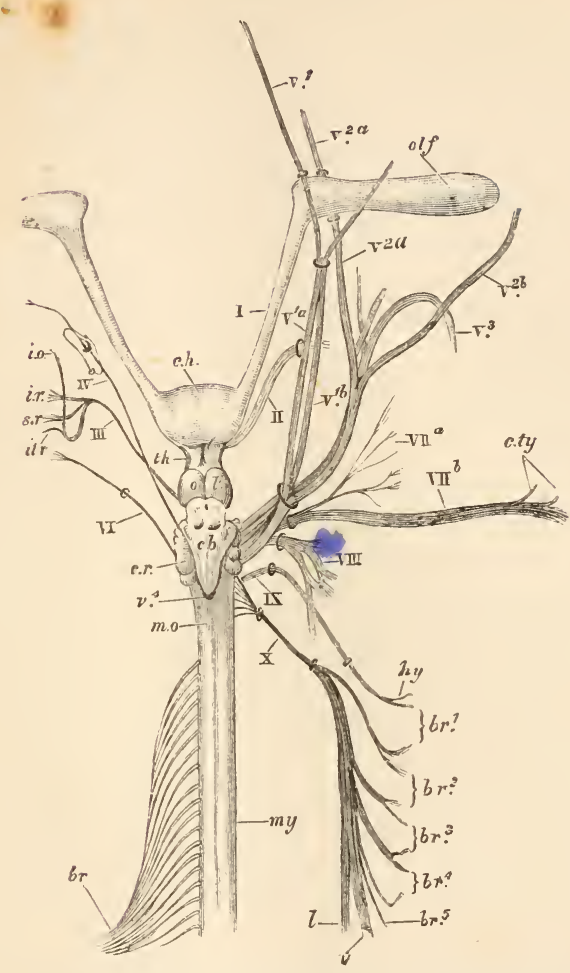

FIG. 22.-Raja nasuta. Dorsal view of the brain and anterior part of the spinal cord, with the cerebral and anterior spinal nerves (half nat. size).

On the left side the 2 nd, 5 th, and 7 th-Ioth cerebral nerves are removed, and on the right side the 3 rd, $4^{\text {th }}$, and 6 th. The spinal nerves are shown only on the left side: the distal end of the left olfactory lobe is not shown.

Central Nervous System. my, spinal cord: m.o, medulla oblongata: $v$. 4 , fourth ventricle : $c . r$, curpora restiformia : $c b$, cerebellum : $0 . l$, optic lobes : th, thalamencephalon : c.h, prosencephalon : olf; olfactory lobes.

Nerves. $I$, olfactory : II. optic : III, oculomotor: i.r, branch of oculomotor to internal rectus: s.r, to superior rectus: it.r, to inferior rectus : $i .0$, to inferior oblique: $I V$, patheticus : $V^{\text {ra }}$, dorsal, 
and $V^{\mathrm{rb}}$, ventral ramus of $V^{\mathrm{r}}$ orbito-nasal nerve : $V^{\text {2a }}$, palato-nasal nerve : $V^{2 \mathrm{~b}}$, maxillary nerve $: V^{3}$, mandibular nerve $: V^{1} I^{\mathrm{a}}$, palatine branch of portio dura: $V I I^{\mathrm{b}}$, hyomandibular branch : c.ty, chorda tympani : $V I I I$, auditory nerve : $I X$, glossopharyngeal nerve : $X$, vagus : $h y$, branch of glossopharyngeal to hyoid arch: $b r$. I, branches of glossopharyngeal and vagus to Ist branchial arch: $b r .2, b r .3, b r .4$, branches of vagus to 2 nd, 3 rd, and 4 th branchial arches: $v$, ventral (gastric and cardiac) branch of vagus : $l$, lateral branch of vagus : $b r$, brachial plexus.

forward continuations of the ventral pyramids of the medulla oblongata.

174. The thalamencephalon (Figs. 22, 24, 25, th), a small division of the brain just anterior to the optic lobes: it consists of two lateral masses of nervous matter, the thalami optici, between which is a space, covered only by pia mater, the thalamocœle or third ventricle $(v \cdot 3)$.

175. The lobi inferiores (Figs. 23-25, l.i) paired ovoidal bodies on the under surface of the thalamencephalon.

I76. The pituitary body or hypophysis cerebri (Figs. 23-25, $p t$ ), a rounded structure on the ventral surface of the brain, behind the lobi inferiores and attached to a backward prolongation of the thalamencephalon called the infundibulum; with it is connected anteriorly the median artery formed by the union of the two internal carotids ( $\$$ I60).

I77. The saccus vasculosus (Figs. $23-25$, s.v, s. $v^{\prime}$ ). a thin-walled hollow body, consisting of three lobes, two large and paired, situated one on either side between the pituitary body and the lobi inferiores, and a median azygos lobe lying in the groove between the lobi inferiores.

I73. The prosencephalon (Figs. 22-25, c.h), a large transversely elongated mass, in front of the thalamencephalon and representing the fused cerebral hemispheres.

I 79. The olfactory lobes (Figs. 22-25, I), two long, nerve-like bodies, given off from the antero-lateral angles 
of the prosencephalon, and passing forwards and slightly outwards to the olfactory foramina $(\$ 32)$. Their further course will be seen at a later stage ( $\$ 206)$.

I80. By lifting up or removing the cerebellum, the whole extent of the fourth ventricle or myelocœle (Figs. 22 and $24, v .4$ ) is seen : viewed from above it has a triangular shape, the base being forwards, and the apex (calamus scriptorius) backwards, as already seen.

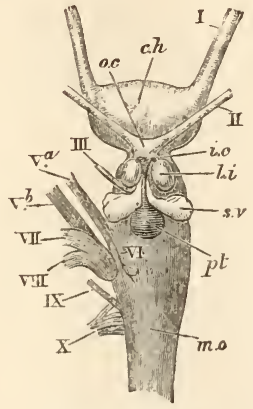

FIG. 23. - Raja batis. The brain from beneath (half nat. size).

Only the proximal portions of the olfactory lobes are shown. m.o, medulla oblongata : $p t$, pituitary body: s.v, saccus vasculosus : $l . i$, lobi inferiores : i.c, internal carotid artery : o.c, optic chiasma : c.h, prosencephalon: $I-X$, cerebral nerves.

I \& I. By carefully cutting away the optic lobes, the fourth ventricle is seen to be continuous with a cavity in the mesencephalon, the aqueduct of Sylvius or mesocœle (Fig. 24, aq. s), which sends lateral prolongations into the optic lobes. Anteriorly the mesocoele is seen to be continuous with the thalamocœle $(i, 3)$.

I 82. By cutting into the prosencephalon, it is seen to be solid, lateral ventricles (prosocœles) being absent.

183. From the under surface of the thalamencephalon arise the two large optic (second cerebral) nerves (Figs. 22. 
23, and 25, II) : their proximal ends are fused together to form an optic chiasma (Fig. 23, o.c). Each nerve passes outwards and forwards to the corresponding optic foramen $(\$ 30)$.

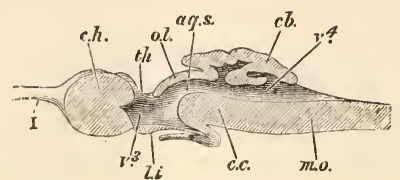

FIG. 24.-Raja batis. Longitudinal vertical section of the brain (half nat. size).

m.o, medulla oblongata : $v .4$, fourth ventricle : $c b$, cerebellum: c.c, crus cerebri : o.l, optic lobe: aq.s, aqueduct of Sylvius : th, thalamencephalon: $v$. 3 , third ventricle: $l . i$, lobus inferior: $c . h$, prosencephalon.

I 84. The third pair of nerves (oculomotores, Figs 22 , 23,25 , III), arising one on either side of the base of the brain from the crura cerebri, a little behind the lobi inferiores, and passing outwards and forwards to their foramina in the side walls of the skull ( $\$ 30$ ).

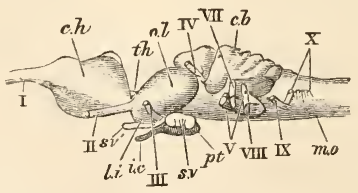

FIG. 25.-Raja batis. Side view of the brain (half nat. size). m.c, medulla oblongata : $c b$, cerebellum: i.c, internal carotid artery: $o . l$, optic lobe: $p t$, pituitary body: $s v, s . v^{\prime}$, saccus vasculosus : th, thalamencephalon : $c, h$, prosencephalon: $I-X$, cerebral nerves.

I 85. The fourth pair of nerves (pathetici, Figs. 22, 23, $25, I V$ ), delicate threads which proceed from beneath the antero-lateral regions of the cerebellum, outwards and forwards to their foramina in the side walls of the skull (\$30). 
186. Two large bundles of nerves proceeding one from either side of the medulla oblongata, beneath the restiform

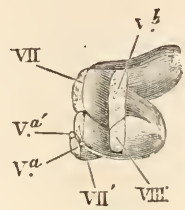

Fig. 26.-Raja nasuta. External view of the roots of the $5^{\text {th, }} 7^{\text {th }}$, and 8th cerebral nerves of the left side (nat. size).

$V^{\mathrm{a}}, V^{\mathrm{a}}$, ventral or anterior roots of the trigeminal: $V^{\mathrm{b}}$, dorsal or posterior root of the same : VII, VII', roots of the portio dura: $V I I I$, root of the allditory nerve.

bodies. Each bundle is seen to consist of an anterior smaller and a posterior larger fasciculus. The former (Figs. 23,26 , and $27 V^{a}$ ) goes to form the inferior ramus of the

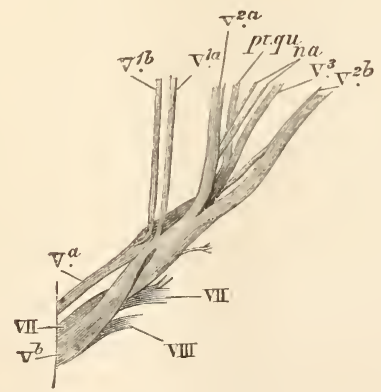

FIG. 27.-Raja nasuta. The principal branches of the 5 th, 7 th, and 8th cerebral nerves (nat. size).

$V^{\mathrm{a}}$, ventral and $V^{\mathrm{b}}$, dorsal root of the fifth: $V^{\mathrm{ra}}$, dorsal, and $V^{\text {ib }}$, ventral ramus of the orbito-nasal nerve: $V^{\text {2a }}$, palato-nasal nerve : $V^{2 b}$, maxillary nerve : $V^{\gamma}$, mandibular nerve : $p t . q u$, nerve to upper jaw . $n a$, nerves to the region of the nostrils: VII, portio dura: VII $i$, auditory nerve.

first division of the fifth nerve, and the main part of its third division, besides contributing fibres to the second 
division: the latter $\left(V^{\mathrm{b}}, V I I, V I I I\right)$ contains the remainder of the fifth, the seventh, and the eighth nerves.

The fifth, seventh, and eighth nerves arise altogether by seven roots. Two of these, a smaller dorsal (Fig. 26, $V^{a^{\prime}}$ ) and a larger ventral $\left(V^{a}\right)$ go to form the anterior fasciculus already mentioned, both arising ventrolaterally from the medulla. The five roots forming the posterior fasciculus divide naturally into an anterior and a posterior set. The anterior set consists of two roots, a dorsal $(V I I)$ and a ventral ( $\left.V^{\prime} I I^{\prime}\right)$, together forming the seventh nerve : the posterior set consists of three roots, a dorsal $\left(V^{\mathrm{b}}\right)$, arising along with the dorsal root of the seventh, and two ventral, arising close together: the uppermost ventral root unites immediately with the dorsal root to form the remainder of the fifth nerve $\left(V^{\mathrm{b}}\right)$ : the lowermost ventral root $(V I I I)$ constitutes the eighth nerve.

187. The sixth pair (abducentes, Figs. 22 and $23, V I$ ) arise from the under surface of the medulla oblongata, internal to the eighth. They are very fine nerves, and are easily detached with the pia mater.

I88. The ninth pair (glossopharyngei, Figs. 22, 23 , $25, I X)$, a small pair arising from the sides of the medulla oblongata, and passing outwards and forwards to enter the auditory capsule at about the centre of its inner surface $(\S 33)$.

189. The tenth pair (vagi or pneumogastrici, Figs. 22, $23,25, X)$, arise by several roots from the lateral regions of the hinder part of the medulla oblongata, and pass outwards and backwards to leave the cranial cavity by a foramen in the posterior part of the inner surface of the auditory capsule (\$33).

XLI. Remove the skin from the dorsal surface of the head, on both sides of the cranial cavity, and by carefully dissecting away the connective tissue from the eye and surrounding parts, make out

190. The dorsal ramus of the orbito-nasal nerve (first division of the fifth, Figs. 22 and $\left.27, V^{1 a}\right)$ lying close 
against the outer side of the skull wall, and springing from the posterior dorsal root mentioned in $\S \mathrm{I} 86$.

I9I. The superior oblique muscle of the eye, passing from its origin at the anterior part of the skull wall to its insertion in the antero-superior region of the eye-ball.

I92. The superior rectus muscle, arising from the skull wall, a little in front of the auditory capsule, and passing to its insertion on the postero-superior region of the eye-ball.

I93. The internal rectus, arising immediately in front of the superior rectus and passing to the front part of the eye-ball, beneath the superior oblique.

I94. The external rectus, arising just behind the superior rectus and passing almost directly outwards to the posterior region of the eye-ball.

I95. The fourth nerve (Fig. 22, IV), leaving the skull by its numerous foramina, and spreading out in a fan-like manner on the dorsal surface of the superior oblique muscle.

I96. The third nerve (Fig. 22, III), which, after leaving the skull, sends branches to the superior (s.r) and internal (i.r) recti, and then curves round the posterior edge of the former: its further course will be seen at a later stage ( $\$ 203$ ).

197. The sixth nerve (Fig. 22, VI), leaving the skull along with the fifth and seventh, and being distributed to the external rectus muscle.

XLII. Remove the superior oblique and the superior and external recti. Make out

198. The ventral ramus of the orbito-nasal nerve (Figs. 22 and $27, V^{\mathrm{r}}$ ) springing from the anterior fasciculus mentioned in $\S \mathrm{I} 86$ : after leaving the skull by the trigeminal foramen, it passes over the external rectus, under the 
superior and internal recti, over the peduncle of the eye ( $\$ 200$ ) and the optic nerve (\$ 199), under the superior oblique, and finally unites with the dorsal ramus as it perforates the nasal capsule ( $\$ 205)$.

199. The optic nerve (Fig. 22, II), which, after leaving the skull, passes almost directly outwards to the hinder region of the eye-ball. Its extra-cranial portion is invested with a strongly pigmented sheath.

200. The ophthalmic peduncle, an irregular stalk of cartilage, articulated at its proximal end with the skull wall behind the optic foramen, and at its distal end with a knob on the sclerotic coat of the eye.

201. The inferior oblique muscle of the eye, running parallel with the superior oblique, to the antero-inferior region of the eye.

202. The inferior rectus, arising with the other recti, and passing parallel with the superior rectus to its insertion on the inferior region of the sclerotic.

203. The remaining course of the third nerve (Fig. 22, III): after curving round the superior rectus, it passes forwards and sends branches to the inferior rectus (it.r) and inferior oblique $(i .0)$.

XLIII. Remove successive slices from the nasal capsule, until the following structures are brought into view.

204. The strongly pigmented nasal sac, filling up the cavity of the nasal capsule.

205. The orbitc-nasal nerve (Fig. 22, $V^{1}$ ) which, just before the junction of its two rami, enters the nasal capsule, and divides into two branches, a large one which passes along the outer edge of the rostrum, and a small one, which passes outwards and forwards over the roof of the nasal sac. 
206. The distal ends of the olfactory lobes (Fig. 22, olf), each forming a slightly convex white band on the dorsal surface of the corresponding nasal sac.

XLIV. Remove one of the nasal sacs, and note 207. Its cup-like form, the cavity being open below.

208. The ridges into which its lining of mucous membrane is raised: they are arranged in two rows at right angles to a central ridge.

XLV. Remove the eye with its remaining muscles, and set it aside for future examination. ${ }^{1}$ Dissect out

209. The second division of the fifth nerve (Figs. 22 and 27 , $\left.V^{2}\right)$, arising mainly from the posterior fasciculus mentioned in $\S \mathbf{I} 86$, but also receiving fibres from the anterior fasciculus: it passes forwards and slightly outwards, and divides into two chief branches, an internal, the palato-nasal nerve $\left(V^{2 \mathrm{a}}\right)$ and an external, the maxillary nerve $\left(V^{2 \mathrm{~b}}\right)$ the former passing directly forwards alongside the cranial wall and beneath the eye muscles, and the latter passing outwards and forwards towards the antorbital cartilage (§35).

2 I0. The third division of the fifth, or mandibular nerve $\left(V^{3}\right)$, arising mainly from the anterior, but receiving fibres from the posterior fasciculus: it runs parallel with the common trunk of the second division as far as the bifurcation of the latter, and then takes a course internal to and nearly parallel with the maxillary nerve.

The three divisions of the fifth nerve supply between them all the anterior part of the head : the orbito-nasal goes to the dorsal region of the rostrum and nasal capsule, and to the gelatinous tissue in their neighbourhood: the palato-nasal to the ventral region of the same parts, as well as to the fronto-nasal process and the nostrils; it also sends

1 The eye must be dissected in the fresh condition : for directions see § LII., p. 84 . 
fibres to the upper jaw : the main part of the mandibular nerve goes to the sensory tubes in front of the antorbital cartilage: the mandibular goes to the lower jaw (outer side), but also sends a large branch to the upper jaw. There is, however, endless variation in the distribution of these nerves.

XLVI. Carefully slice away the cartilage forming the roof of the auditory capsule, taking care not to injure the membranous labyrinth ( $\left(2 \mathrm{I}_{3}\right)$; dissect away the connective tissue, \&c., from the hyomandibular, and the muscles on the floor of the orbit, making out

2 II. The hyomandibular nerve, or posterior branch of the seventh (Fig. 22, $V I I^{\mathrm{b}}$ ) : after leaving the cranial cavity it curves round the anterior border of the auditory capsule, and passes almost directly outwards, over the hyomandibular cartilage and behind the jaw muscles, breaking up at last into a number of fibres which are distributed to the large sensory tubes situated in front of the gills. As the hyomandibular nerve passes the jaw muscles, it gives off two small branches (c.ty), which represent the chorda tympani of the higher animals, and are distributed to the inner surface of the lower jaw.

212. The palatine nerve (Fig. 22, $V I I^{2}$ ), or anterior division of the seventh, which separates from the common root of the seventh soon after its exit from the skull, passes in front of the spiracle, and breaks up into a number of branches, some of which are distributed to the mucous membrane of the mouth and others to the pseudobranchia.

2r3. The membranous labyrinth (Fig. 28) or internal ear, a delicate apparatus enclosed within the auditory capsule, and consisting of a large sac or vestibule $(v)$, and three semicircular canals, one of which is anterior and vertical (a.s.c), another posterior and vertical (p.s.c), and the third horizontal (h.s.c). 
The vestibule is a rounded sac with two small diverticula, containing an otolithic mass which consists of minute calcareous particles bound together by gelatinous connective tissue : from its dorsal surface proceeds a canal, the aqueductus vestibuli $(a q . f)$, which passes through the roof of the auditory capsule, and dilates between the latter and the external integument into a small sac, lined with strongly pigmented epithelium ; this opens on the surface of the head by a minute aperture. The canals form nearly complete circles, the posterior being the largest of the three. Each is dilated at one part of its course into an ampulla (a). The posterior canal communicates with the vestibule in an unusual manner, namely by a short distinct tube $(x)$.

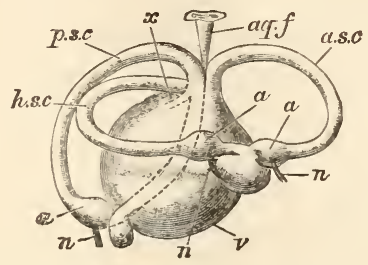

FIG. 28.-Raja batis. The right membranous labyrinth, seen from the outer side (nat. size).

$v$, vestibule: aq.f, aqueductus vestibulit: a.s.c, anterior, p.s.c, posterior, and h.s.c, horizontal semicircular canals : $a, a, a$, ampullæ : $x$, side tubulure by which the posterior canal communicates with the vestibule : $n, n, n$, branches of auditory nerve.

XLVII. Clear away the skin and muscles from the dorsal surface of the anterior vertebral plate and from that of the gills. Dissect out

2 I 4. The vagus or tenth nerve (Fig. 22, $X$ ), which, after leaving the skull by the vagus foramen, passes directly backwards between the inner boundaries of the gills and the lateral ridge of the anterior vertebral plate, giving off the branchial nerves as it goes, to the front edge of the propterygium, where it divides into two chief branches, a ventral branch $(X, v)$, to the stomach and heart, and a lateral branch $(X, l)$, which passes between the peritoneum and dorsal 
muscles, on the dorsal side of the spinal nerves (\$222). The branchial nerves (br. $2-5$ ) are four in number, and are given off opposite the four posterior gill clefts. Each one, soon after leaving the vagus divides into two branches, one of which goes to the posterior face of the gill in front of the cleft to which it belongs, the other to the anterior face of the gill next behind. In this way all the gill filaments except those on the posterior face of the hyoid and those on the anterior face of the first branchial arch (first and second demibranchs) are supplied.

2 I5. The glossopharyngeal or ninth nerve (Fig. 22, $I X)$, seen emerging from its foramen, in front of the anterior branchial branch of the vagus : it divides above the first gill cleft and supplies the hyoid half-gill and the anterior halfgill of the first branchial arch (hy, br. I).

216. The brachial plexus (Fig. 22, br), lying in the depression between the median and lateral ridges of the anterior vertebral plate, and formed by the convergence and subsequent union of the first sixteen or eighteen spinal nerves: the common trunk thus formed turns round the posterior edge of the lateral ridge, and passes outwards behind the propterygium to the pectoral fin. Each spinal nerve arises by two roots, a dorsal (Fig. I I, d.r) and a ventral $(v \cdot r)$, the former having a ganglionic enlargement : the two pass separately through the wall of the neural canal (see $\S \S 8$, Io) and unite outside it in the trunk $(t r)$ of the nerve.

XLVIII. Remove carefully the greater part of the membranous labyrinth, cut away the walls of the auditory capsule, and make out

217. The auditory or eighth nerve (Fig. 22, VIII), entering the capsule at its anterior end through the internal auditory meatus, and passing backwards, sends off 
brarches to the vestibule and ampullæ of the semicircular canals (Fig. $28, n$ ).

218. The course of the ninth nerve through the capsule (Fig. 22, $I X$ ) : it enters the latter by an aperture in about the middle of its anterior wall, passes backwards and outwards nearly parallel with the eighth and leaves the capsule in its postero-external region by an aperture already noticed in the skull (\$25).

219. The course of the vagus through the auditory capsule (Fig. 22, X) : leaving the cranial cavity it passes through a canal excavated in the postero-internal wall of the capsule, the outer end of the canal being the vagus foramen (see $\$$ 24, 33).

XLIX. Place the fish in the supine position, find the large sympathetic ganglion mentioned in $\S$ I50, and trace back

220. The sympathetic nerve, which consists of a longitudinal cord on each side of the vertebral column, presenting ganglia at intervals, and connected by rami communicantes to the spinal nerves. As already mentioned, the adrenals or supra-renal bodies are in intimate connection with the sympathetic ganglia.

L. Dissect away the kidneys and the peritoneum from the dorsal wall of the abdominal cavity, and note

$22 \mathrm{I}$. The spinal nerves, passing to the body walls and fins : many of them converge, and exhibit a tendency to the formation of plexuses.

222. The lateral branch of the pneumogastric (Fig. 22, $X, l)$, a longitudinal nerve, lying to the dorsal side of the spinal nerves.

LI. Make a longitudinal vertical section of a brain hardened in spirit, and note

223. The relations of the various divisions of the brain aiready seen, and of the cavities they contain (Fig. 24): the 
latter are seen to form a continuous series, from the myelocœle $(v \cdot 4)$, which is continued behind into the central canal of the spinal cord, through the mesocœle (aq.s) to the thalamocole $(v \cdot 3)$. The latter is further seen to send a small prolongation forwards into the otherwise solid prosencephalon, and another downwards and backwards into the infundibulum.

LII. Make out the chief structures in the eye, first viewing from the outside, then removing successively the sclerotic ( $\$ 224)$, choroid ( $\$ 225)$ and retina (\$2 27) from the flattened dorsal surface.

224. The cartilaginous sclerotic or outer covering of the eye-ball: externally it passes into the transparent fibrous cornea, which is the part exposed to view in the entire animal : internally, the sclerotic is pierced by a small aperture for the passage of the optic nerve, and is raised into a knoblike prominence for articulation with the ophthalmic peduncle $(\$ 200)$.

225. The black choroid lining the sclerotic: at the junction of the latter with the cornea, it passes into the iris, the coloured (yellow and black) part of the eye, seen from the outside through the transparent cornea. The iris is pierced by a central aperture, the pupil, the upper margin of which is produced into a sort of curtain, divided at its free edge into several processes. Between the iris and the cornea is a space, the anterior chamber of the eye, filled with a watery fluid, the aqueous humour.

226 . The crystalline lens, a nearly globular transparent body, situated immediately behind the iris.

227. The retina, a delicate, greyish-white membrane, lying immediately within the choroid, from which it is readily detached except at the place of entrance of the optic 
nerve. Externally (i.e. towards the cornea) it ends at the junction of the choroid and iris.

228. The vitreous humour, a gelatinous substance, fill= ing the whole posterior chamber of the eye, or space enclosed between the retina and the inner surface of the lens and iris. 


\section{THE COD.}

The Codfish (Gadus morrhua).

The Whiting (G. merlangus).

The Haddock (G. aglefinus). ${ }^{1}$

\section{A.-THE SKELETON.}

I. A cod's skeleton is readily prepared by placing the fish, after removal of the viscera, into boiling water for a few minutes, and thus stripping off the flesh and other soft parts. By this process the bones are obtained separate from one another, with the exception of those forming the brain-case, the high temperature causing gelatinization of the connective tissue which unites them. The bones should be removed one by one and placed out in order, to dry, each being as far as possible identified and its relations to surrounding bones determined before removal. As this is a matter of considerable difficulty in the case of the bones of the head, it is advisable to prepare a second skull

1 These are the three commonest species of the genus Gadus brought to the English markets : the differences between them are comparatively unimportant (see $\S 8,1$ \&, 19, 72, 80, 85). 
by carefully dissecting away the muscles, \&c., while fresh, without either boiling or maceration, the bones being kept together in their natural position by their ligaments. Of this skull it is as well to make a longitudinal vertical section by sawing through the brain-case a little to one side of the middle line and cutting through the mandibular and hyoidean symphyses ( $\$ 55_{1}$ and $5_{2}$ ): from the worst side, the jaw apparatus should be removed from the brain-case by disarticulating the palatine ( $\$ 46$, Fig. 29, $p a$ ) and the hyomandibular ( $\S 40$, Fig. 29, $/ 2 m)$. In the first skull the bones of the brain-case may be separated from one another by boiling for a considerable time and then gently puliing them asunder.

It is advisable to examine the second or entire skull before drying, so as to see its cartilaginous portions (\$\$ 47, 49, \&c.). ${ }^{1}$

II. In the skeleton as a whole note the following regions :-

I. The vertebral column, consisting of $(a)$ truni vertebræ, bearing movable ribs which do not unite

${ }^{1}$ Owing to the small amount of cartilage left in the adult cod's skull, the beginner will find some difficulty in seeing the relation between it and that of the skate. A useful intermediate type is furnished by the salmon or trout, in which there is a cartilaginous brain-case, quite readily comparable with that of the skate but containing the occipital and otic bones, basi- and pre-sphenoid, as endogenous ossifications : the remaining homologues of the bones of the cod's brain-case (parietals, frontals, parasphenoid, vomer, \&c.) are seen to be membrane-bones, easily detachable without injury to the cartilage (see Parker and Bettany, Morphology of the Skull, p. 66). The skull of the salmon or trout should be prepared like that of the cod, by b iling ; the braincase, Meckel's cartilages, \&c., may be preserved by the glycerine jelly process, described on p. 2. 
below, and (b) caudal vertebræ, with complete inferior arches.

2. The skull, consisting of $(a)$ the brain-case, formed of a number of bones firmly united by suture ; $(b)$ a number of more or less loosely attached bones, in relation with the brain-case, and constituting the skeleton of the upper and lower jaws and suspensorium, the hyoidean apparatus or tongue-bones, and the gill-covers; and (c) the branchial arches or bony framework which supports the gills.

3. The bones of the median fins, namely, the three dorsal fins, the two anal fins, and the caudal fin (see § 94).

4. The bones of the pectoral fins, or fore-limbs, and of the shoulder girdle to which they are attached (§ 95).

5. The bones of the pelvic fins, or hind-limbs, and of the hip-girdle to which they are attached $(\S 96)$.

III. Examine a vertebra from about the middle of the trunk region, and make out

6. The centrum or body of the vertebra, a short bony cylinder with deeply concave anterior and posterior ends; the bi-convex spaces between adjacent centra are filled in the recent state by a gelatinous substance, the remains of the embryonic notochord.

7. The neural processes, two plates arising vertically one from each side of the upper surface of the centrum, near its anterior end, and uniting with one another to form the neural arch, from the vertex of which the long slender neural spine springs and takes a direction upwards and slightly backwards. From the anterior edge of each neural process a somewhat triangular projection is continued forwards, and answers to the anterior zygapophysis of 
the higher vertebrata : there is no true posterior zygapophysis, but from the hinder end of the centrum arises on each side a small vertical process, which fits outside the anterior zygapophysis of the vertebra next behind.

8. The transverse processes, large outstanding plates of bone, which spring one from each side of the centrum, and pass outwards, downwards, and backwards.

The transverse processes are proportionally much larger in the Haddock than in either of the other species.

IV. In the rest of the trunk region, the following are the chief points to be verified :-

9. In the first vertebra or atlas the centrum is very short from before backwards, the neural spine is vertical, the transverse processes are absent, and the anterior zygapophyses are very large and come into relation with the posterior part of the skull (exoccipital bone, § 22); immediately beneath each zygapophysis is a small articular facet for articulation with a corresponding facet on the exoccipital. The union between the skull and the atlas is thus much more intimate than that between any two vertebræ, and in consequence the atlas is, in preparation, often left attached to the skull.

Io. In the next three or four vertebræ a gradual transition is seen between the characters of the atlas and those of the typical trunk vertebra described in $\S \S 6-8$.

II. The transverse processes in the anterior part of the trunk region look almost directly outwards; proceeding towards the caudal region, they gradually increase in size and come to look more and more downwards and backwards, until in the last trunk vertebra they nearly meet.

I 2. Between the ventral or proximal ends of contiguous neural arches, spaces are left : these are the in tervertebral 
foramina; they serve for the transmission of the spinal nerves.

I3. The ribs, slender, flattened bones, articulated to the distal ends of the transverse processes and curving outwards and downwards.

14. The inter-muscular bones, shorter and slenderer than the ribs, but otherwise resembling them : they are articulated one to each transverse process on its dorsal side and a short distance from its distal end, and curve upwards and outwards.

V. In the caudal vertebræ note .

15. The hæmal arch, formed by the union in the middle ventral line of two hæmal processes, springing one from each side of the ventral surface of the centrum near its anterior end ; from the point of union a hæmal spine is given off and passes clownwards and backwards. The hæmal processes give rise to anterior zygapophyses like those on the neural arches, and similarly articulating with small processes from the centrum of the vertebra next in front.

I6. In the anterior caudal region the hæmal are very much larger than the neural arches, but passing back the former diminish progressively until there is no difference of importance, either in the size of the arches or the length of the spines, between the neural and hæmal aspects of the vertebræ.

I 7. Following the last undoubted caudal vertebra is the small fan-shaped hypural bone, which together with the somewhat flattened posterior neural and hæmal spines supports the tail-fin.

VI. In the median fins make out

I8. The interspinous bones of the dorsal and anal fins, alternating with the neural and hæmal spines respectively, and attached to them by fibrous tissue. 
In the Whiting the anterior anal fin extends in front of the caudal region, so that most of its interspinous bones are unconnected with the vertebræ and lie free amongst the muscles.

I9. The fin-rays, attached, in the case of the dorsal and anal fins, at their proximal ends to the interspinous bones, and forming the actual skeleton of the fins; each is a delicate, rod-like bone, transversely jointed and flexible at its distal end ("soft fin-rays"). In the caudal fin the fin-rays come into direct relation with the hypural bone and the posterior neural and hæmal spines.

In the Codfish there are 13 rays to the first dorsal fin, 16 to 19 to the second, $17-19$ to the third, $18-19$ to the first anal, and $17-18$ to the second. The formula for the fin rays is therefore, D. I3 $\mid$ r6-19| I7-19. A. 18-19/17-19. In the Haddock the formula is D. I4-I6 $|20-24| 19-2$ I. A. 24-25|2I-2 2; and in the Whiting D. $13-14|20-23| 20-2$ r. A. $33-35 \mid 22-24$.

VII. In the brain-case make out the following points :20. The single concave occipital condyle, for articulation with the centrum of the atlas: it is the hinder face of the basi-occipital bone (Fig. 29, 6.0), which forms the posterior part of the base of the skull, ending in front by a thin jagged edge.

$2 \mathrm{I}$. The foramen magnum or occipital foramen, a large aperture immediately above the occipital condyle, for the passage of the spinal cord into the brain.

22. The ex-occipitals (e.o), two irregular bones forming the lateral boundaries of the foramen magnum and articulating below with the dorso-lateral edges of the basi-occipital. Each ex-occipital is perforated by an aperture for the exit of the vagus nerve, and presents on its posterior border an articular facet for the corresponding surface already seen on the neural arch of the atlas ( $§ 9)$.

23. The supra-occipital (s.o), a large bone bounding 
the foramen magnum above, and produced dorsally into a median, laterally-compressed crest, the occipital spine. The basi-, ex-, and supra-occipitals together form the occipital segment of the skull.

24. Externally to the occipital region, the brain-case is produced on either side into a large out-standing mass, the auditory capsule, which presents above two well-marked processes-an inner, the epiotic process, short and subtetrahedral in form; and an outer, the parotic process, which is long, and projects backwards and outwards. The median occipital spine and the paired epiotic and parotic processes form the five projections which are so marked a feature in the postero-dorsal region of the skull.

25. The epiotic process forms nearly the whole of the small epiotic bone (ep.o), the postero-internal ossification of the auditory capsule.

26. The parotic process is constituted by two bones, which are seen to meet in an irregular suture on its outer surface. The uppermost of these is the pterotic (pt.o), or postero-dorsal ossification of the auditory capsule, the lower the opisthotic (op.o), or postero-ventral ossification : the latter articulates behind with the ex-occipital, and below with the basi-occipital, and is perforated near its posterior border by a foramen for the exit of the glossopharyngeal nerve.

27. The prootic (pr.o), a large bone, articulating behind with the opisthotic and forming the antero-ventral ossification of the auditory capsule: its anterior border is deeply notched for the passage of the fifth and seventh cerebral nerves.

28. The sphenotic (sp.o), the antero-dorsal ossification of the auditory capsule, a large, irregular bone, articulating behind with the pterotic and below with the prootic. 
29. A large articular surface ( $y$ ) on the side wall of the auditory capsule, formed in front by the sphenotic, behind by the pterotic; it is covered with cartilage in the recent state and gives articulation to the hyomandibular (§ 40).

30. The frontals $(f r)$ united with one another in the middle line into a single large shield-shaped bone which forms the greater part of the roof of the brain-case, articulating behind with the supra-occipital in the middle line, and with the sphenotics externally.

3 r. The parietals $(p)$, two flat bones, completing the roofing-in of the brain-case, being wedged in, one on either side, between the supra-occipital internally, the sphenotic and pterotic externally, the frontal in front and the epiotic behind. On the dorsal surface of the parietal is a spine-like process, beneath which is a foramen for the exit of the cutaneous branch of the fifth nerve $(\S 98)$.

32. The alisphenoids (al.s), two small bones, situated in the side walls of the brain-case, and articulating with the prootic behind and below, and the frontal and sphenotic above.

33. The parasphenoid ( $p a . s$ ), a iong, stout, median bone, forming the greater part of the base of the skull: posteriorly it widens out, underlaps the basi-occipital and articulates with the prootics: both in front and behind it thins out considerably and ends in a jagged edge.

34. The vomer $\left(z^{\prime}\right)$, a median bone, forming the anterior part of the base of the skull : it ends in front in a strong transversely curved edge, beset with teeth on its lower surface $(\S \mathrm{I} 6 \mathrm{I})$, posteriorly it is pointed and underlaps the parasphenoid.

35. The mesethmoid (m.e), a median bone situated immediately above the vomer: it is truncated in front, and 
sends off backwards a long process which fits into a deep notch in the frontals.

36. The parethmoids $(p . e)$, irregular paired bones forming large lateral projections at the anterior end of the brain-case, representing ossifications of the olfactory capsules. Each presents on its outer surface an articular cavity $(x)$ for the palatine ( $\$ 46)$.

37. A vacuity on each side between the mesethmoid, the parethmoid, and the frontal, and serving to lodge the nasal sac (\$ I 80$)$.

38. The absence of side walls to the skull except in the olfactory and auditory regions.

39. A longitudinal section shows that in the dried skull there is free communication between the cavity for the brain and those for the auditory organs.

VIII. In each side of the upper jaw and suspensorium make out

40. The hyomandibular $(\mathrm{hm})$, a large bone articulating with the facet $(y)$ afforded by the sphenotic and pterotic by a prominent rounded head $\left(y^{\prime}\right)$ : it sends off a long backward process for articulation with the opercular, and presents on its outer surface a prominent, downwardly directed ridge serving for muscular attachments, and perforated anteriorly by two foramina for the seventh nerve. At its lower end the hyomandibular ends in front in a jagged triangular process, behind in a straight edge.

4I. The symplectic (sy), a long, somewhat triangular bone, having a short dorsal edge united by synchondrosis with the straight lower border of the hyomandibular, and a pointed ventral process which fits into a groove on the inner face of the quadrate $(\S 42)$. In the natural position of the bones, the symplectic, as well as a considerable portion of 
the hyomandibular and quadrate, is hidden by the preopercular (\$ 64).

42. The quadrate $(q u)$ a bone of very irregular form, presenting on its inner surface a deep groove for the reception of the symplectic, and ending below in a saddleshaped articulation for the lower jaw.

43. The meta-pterygoid (m.pt), a thin lamina of bone, united by synchondrosis with the straight part of the dorsal border of the quadrate, and fitting closely against the anterior border of the hyomandibular and symplectic.

44. The pterygoid ( pt), a long, flat, irregular bone, with a thickened ventral border: it articulates by suture with the anterior edge of the quadrate and meta-pterygoid, and takes a direction forwards, and slightly upwards and inwards.

45. The meso-pterygoid (ms.pt), a very thin bony lamina, fitting against the upper edge of the pterygoid.

46. The palatine $(p a)$, an irregular bone, consisting of a flattened posterior portion, and of a stout, rod-like, incurved anterior portion; at the junction of its two parts, the bone presents on its upper surface an articular facet $\left(x^{\prime}\right)$ for articulation with the parethmoid ( $\left.\$ 36\right)$. Posteriorly the palatine ends above in a straight edge, and below in a long jagged process which articulates with the pterygoid.

47. The premaxilla ( $p . m x)$, a stout, curved, rod-like bone forming the gape of the upper jaw, produced at its upper or inner end into a strong upward process, and covered on its oral surface with close-set curved teeth.

The two premaxillæ abut at their dorsal (inner) ends against a large nodule of cartilage, which is not seen in the dried skull.

48. The maxilla ( $m x$ ), lying behind and parallel with the premaxilla: its anterior (dorsal) somewhat expanded 
end articulates with the vomer: its posterior (or ventrai) flattened end reaches nearly as far back as the quadrate. It does not enter into the gape.

IX. Note the following bones in each ramus of the lower jaw :-

49. The articular (ar), a large bone articulating by a saddle-shaped surface with the quadrate: it is thick and strong posteriorly, and produced into a long pointed process anteriorly. On its inner surface is a groove in which, in the recent condition, Meckel's cartilage lies: this latter is rod-like, about $2-5 \mathrm{~mm}$. in diameter, and pointed at its anterior end, which projects beyond that of the articular.

50. The angular (an), a small stout bone attached to the postero-inferior angle of the articular.

-5 $\mathrm{I}$. The dentary $(d)$, a large bone forming the main part of the lower jaw, and curving inwards to join its fellow of the opposite side, to which it is united by synchondrosis. Its posterior edge is deeply notched for the reception of the articular, and it contains a cavity open behind, in which Meckel's cartilage and the mandibular nerves run.

$\mathrm{X}$. Note in the hyoidean apparatus

52. The two paired halves or cornua of the apparatus, large, laterally-compressed bones, lying, in the natural position of the parts, within the rami of the mandible, and uniting with one another by a median symphysis a short distance behind the mandibular symphysis.

53. The connection of each hyoidean cornu with the corresponding suspensorium by a short rod of bone, the interhyal (i.hy), which articulates on the one end with the dorsal extremity of the cornu, and on the other with the cartilaginous interval between the hyomandibular and symplectic. 
54. The composition of the hyoidean cornua: each consists of a dorsal, somewhat triangular bone, the epi-hyal (ep.hy), articulating at its apex with the inter-hyal, and at its base united by suture to a second bone, the cerato-hyal (c.hy), which forms the main part of the cornu, and is connected ventrally with two small ossifications, the hypohyals $(h . / h y)$.

55. The branchiostegal rays (br.r), seven curved rods of bone, articulated to about the upper three-fourths of the posterior edge of the cerato-hyal : the four superior rays are connected with the outer face, the three inferior with the inner face of the cerato-hyal.

56. The uro-hyal or basi-branchiostegal (u.hy), a median vertical plate of somewhat triangular form, interposed between the hypo-hyals of opposite sides.

XI. In the branchial arches, make out

57. The superior pharyngeal bones, two large irregular bones, covered inferiorly with teeth, which lie, in the entire fish, in the roof of the pharynx. These represent the coalesced dorsal elements or pharyngo-branchials of the four anterior branchial arches.

58. The epi-branchials, four slender bones standing out horizontally from the outer edge of each superior pharyngeal bone.

59. The cerato-branchials, four larger bones on each side, forming the main part of the four anterior branchial arches: each is articulated at its dorsal end with the outer (ventral) extremity of the corresponding epi-branchial, and takes a direction forwards, downwards, and slightly inwards.

6o. Each cerato-branchial is articulated below with a hypo-branchial ossification, which passes forwards and inwards.

6r. The basi-branchial, a median ventral ossification, 
common to the first three arches, the hypo-branchials of which articulate with it laterally : the hypo-branchials of the fourth arch are united with one another in the middle ventral line by cartilage.

62. The rudimentary fifth branchial arches, each consisting of a single bone-the inferior pharyngeal bone - which is beset on its dorsal face with teeth, and in the entire fish, bites against the superior pharyngeal (\$§ I62, I63).

XII. In the skeleton of the gill-cover or operculum, note the following four bones on each side :-

63. The opercular (Fig. 29, $o p$ ), a flat bone, bifid posteriorly and having on its anterior border a facet for articulation with the posterior or opercular process of the hyomandibular.

64. The pre-opercular $(p .0 p)$, a large bone with an evenly curved anterior border produced at one place into an irregular process, and a jagged posterior border; its outer face is produced into a prominent ridge for attachment of muscles. In the natural position of the parts it fits closely against the outer face of the hyomandibular, symplectic, and quadrate.

65. The sub-opercular (s.op), a flat plate, somewhat rounded below and pointed above: and lies immediately beneath the opercular.

66. The inter-opercular (i.op), an irregular bone, articulated at its upper and posterior end with the subopercular, and at its opposite extremity connected by ligament to the angle of the mandible.

XIII. There still remain a few loosely attached bones in connection with the brain-case, chiefly in the region of the nose and eyes; these are

67. The so-called nasals (na), delicate scale-like bones, 
grooved for sensory tubes, situated one on either side of the nasal region, to the dorsal side of the parethmoids.

68. The sub-orbitals (s.or), a semicircular chain of bones, lying beneath the eye-ball: the most anterior of the series is large, thin, and triangular in shape, and is the socalled lacrymal (lc); the others are small and five to seven in number. All are grooved for sensory tubes.

69. Two or three small grooved bones of a similar nature occur in relation to the parotic processes.

$\mathrm{XIV}$. In the shoulder-girdle and pectoral fin note on each side

70. The post-temporal, a forked bone, articulating by the inner and larger of its limbs with the epiotic, by the outer and smaller with the parotic process of the skull.

7 I. The supra-clavicle, a stout rod, articulating above with the post-temporal, and fitting below by its bevelled inner surface against the dorsal extremity of the clavicle.

72. The clavicle, a large curved bone, with a thick anterior and a thin posterior border, attached above to the supra-clavicle, and taking a direction at first downwards, then forwards and inwards, so as nearly to meet its fellow of the opposite side in the middle ventral line of the throat, a short distance beyond the uro-hyal.

In the Haddock the anterior (ventral) portion of the clavicle is greatly thickened.

73. The post-clavicle, a slender bony rod, connected with the inner face of the clavicle near its dorsal end, and passing backwards and downwards.

74. The scapula and coracoid, two delicate laminæ attached to the inner face of the clavicle: the upper and smaller of the two is the scapula : the lower, of an irregularly triangular form, is the coracoid. 
75. The brachial ossicles, four small, dice-box shaped bones, articulating with the posterior border of the scapula and coracoid.

76. The fin-rays, attached to the distal ends of the brachial ossicles and having essentially the same character as those of the median fins; they diminish gradually in size from the pre-axial to the post-axial border of the fin (see \$ 95) overlap one another at their proximal ends, and are much frayed out at their distal extremities.

$\mathrm{XV}$. In the hip-girdle and pelvic fins note

77. The pelvis, consisting of the two thin, irregular innominate bones, ${ }^{1}$ united with one another in the middle ventral line, but showing no distinction into the three ossifications of the typical vertebrate os innominatum.

78. The fin-rays, articulating with the postero-external borders of the innominate bones. Owing to the position of the pelvic girdle beneath the throat, the pelvic fins come to be situated anterior to the pectorals.

\section{B.-DIRECTIONS FOR DISSECTION.}

XVI. Make out the following external characters :-

79. The elongated body (Fig. 30), slightly compressed from side to side; the head passing insensibly into the trunk, and the trunk into the tail.

So. The integument, containing numerous small imbricating scales, covered with a layer of thin, slimy, pigmented epiderm, the colour of which varies in different parts of the body, being white below, and greyish or olive, mottled with golden yeliow, above.

${ }^{1}$ It is possible that the so-called innominate bone may represent the basale metapterygii or basipterygium of the Elasmobranch fin ( $\mathrm{p} .4 \mathrm{O}_{8}$ $\S 52$ ), and not a true pelvic girdle. 
In the Haddock the lateral line (see $\S 82$ ), is black, and there is also a blackish blotch below the lateral line, between the pectoral and the first dorsal fins : in the Whiting there is a black spot on the axil of the pectoral fin.

8r. Remove a few scales and examine under a low magnifying power; each is seen to be a flat, rounded plate, composed of concentric laminæ of calcific matter, devoid of bone-cells; its anterior end is embedded in the derm and overlapped by other scales, its posterior end is covered only by epiderm, and presents an even free border ("cycloid" scaies).

82. The lateral line, a horizontal row of peculiarly modified scales, lodging sensory tubes; it passes from the tail forwards, a little above the middle line of the body, and becomes indistinct on the head.

83. The large mouth, at the extreme anterior end of the body, supported below by the mandible (Fig. 29, d) and above by the premaxillæ $(p \cdot m x)$ and maxillæ $(m x)$, of which the latter are behind the former and do not actually enter into the gape. The skin in which the maxilla and premaxilla are contained is so loose as to render the upper jaw slightly protrusible.

84. The teeth are best seen at a later stage ( $\$$ I59-I 63 , p. I 20 ).

85. The barbule (Fig. 30, b), a median filamentous process, hanging from the under side of the lower jaw near the symphysis.

In the Cod the barbule is as long as or longer than the eye : in the IIaddock it is very short : in the Whiting absent.

86. The nostrils (anterior nares) (Fig. $30, n . a$ ), situated a short distance behind the blunt anterior extremity of the snout; these are two small apertures on each side, of which 
the posterior and outer is circular and open, while the anterior and inner is guarded by a flap-like valve.

87. The large eyes $(e)$, devoid of eyelids and covered with a continuous layer of transparent integument.

88. The gill-opening, a large crescentic aperture on each side, extending from under the throat upwards and backwards along the side of the head; it is bounded behind by the shoulder-girdle $(\$ 95)$ and in front by the gillcover or operculum, which is easily distinguishable into two parts. the operculum proper (Fig. $30, o p$ ), supported by the opercular bones ( $\left.\S 6_{3}-56\right)$, and the branchiostegal membrane $(b r . m)$, supported by the branchiostegal rays of the hyoid bone ( $\S 55)$.

89. The gills, seen by lifting up the operculum : they are four in number, and consist of rows of deep red branchial filaments, supported on the four anterior branchial arches $(\$ \$ 57-62)$ : between the gills, as well as in front of the first and behind the last gill, are the branchial clefts, five in number, leading into the cavity of the mouth.

90. The pseudobranchia (rudimentary hyoidean gill). seen as a red patch covered by semi-transparent mucous membrane, on the inner surface of the gill-cover, a little anterior to the dorsal end of the first branchial arch.

9r. The absence of spiracles (p. $43, \S ; 0$ ) and of external anditory apertures.

92. The anus (Fig. $3^{\circ}, a$ ), a somewhat prominent aperture, situated in the middle ventral line, about half-way between the snout and the end of the tail.

93. The genital $(g)$ and urinary (u) apertures, situated on a common elevation of integument, immediately posterior to the anus; they are small apertures, the genital being the more anterior of the two. 


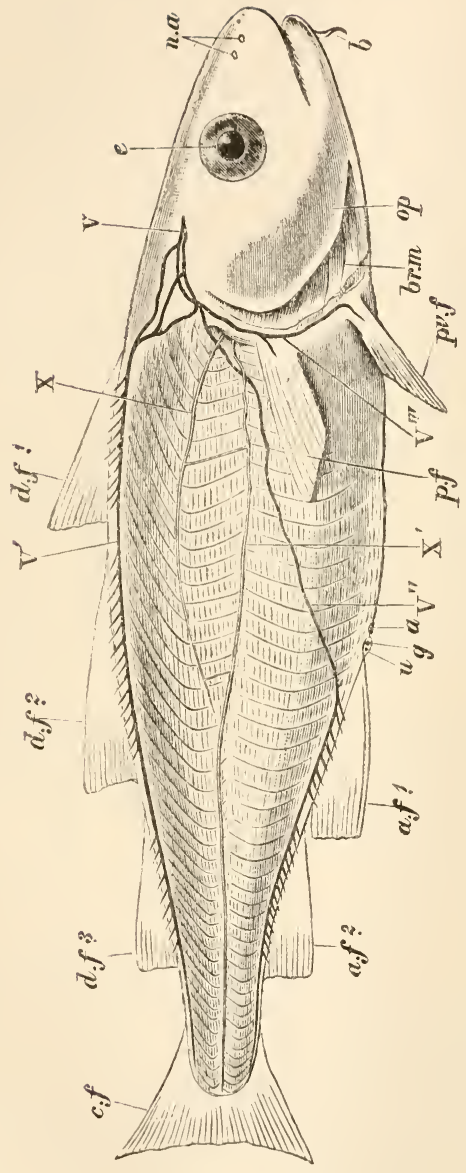

نัष

केष氖芯

ㅈ.

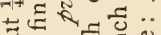

ले

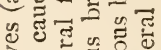

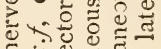

…

z $x-\tilde{z}$

ญี ผ

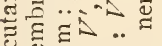

こ悎.

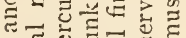

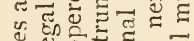

प्ष

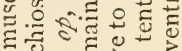

त

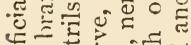

势

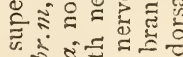

कर 0 ह

Ð

फั0

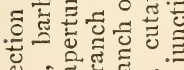

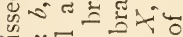

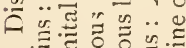

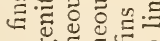

ณं สํ

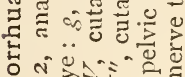

घ

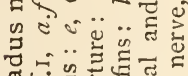

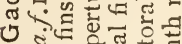

|..7 ส

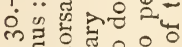

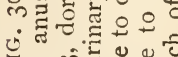

¿ ชิ 
94. The median fins, thin vertical folds of integument supported by bony fin-rays ( $\$$ I9) ; three of them are dorsal in position (dorsal fins, Fig. 3o, d.f. r, d.f. 2, d.f. 3), extending from immediately behind the head to the tail; two are ventral (anal fins, a.f. I, a.f. 2), extending between the urinary aperture and the tail; and one is posterior, the caudal fin $(c, f)$; the latter is apparently quite symmetrical (homocercal), its rays being equally distributed above and below the fleshy lobe in which the tail ends.

95. The pectoral fins (fore-limbs) (Fig. $2, p . f$ ), situated on each side of the anterior region of the trunk, close behind the gill-slits, and about midway between the dorsal and ventral contours. The shoulder girdle to which they are attached (\$\$ 70-74) can be felt immediately behind the gill-siit. If the pectoral fin be made to stand out at right angles to the body, it will be seen to have a dorsal surface looking upwards and backwards, a ventral surface, downwards and forwards, a strong, straight pre-axial border towards the head, and a thin curved post-axial border towards the tail. When left to itself, it lies with its dorsal surface against the side of the body and its pre-axial border looking upwards. 96. The pelvic or so-called ventral fins (hind-limbs) ( $p v \cdot f)$, small fins situated below and slightly in front of the pectorals : the pelvic girdle $(\$ 77)$ to which they are attached can be felt in the angle between the ventral ends of the shoulder girdles. The surfaces and borders of the pelvic fins have the same names as those of the pectorals, and are easily identified.

XVII. ${ }^{1}$ Carefully dissect away the skin from the left side of the body, observing the following :-

1 The following sections ( $\$$ XVII-99) may be omitted until after the dissection of the viscera, and then worked out on the right side of the body. 
97. The body muscles (Fig. 30), distinctly divided into vertical segments or myotomes, separated from one another by septa of connective tissue : each myotome takes a zigzag course, passing, from the middle dorsal line, at first sharply backwards, then gently forwards, then gently backwards, and finally sharply forwards to the middle ventral line. The myotomes are also more or less distinctly divided into dorsal and ventral portions: the dorsal muscles on nearing the head, turn forwards, and are inserted into the frontals; the ventral muscles being inserted into the clavicles.

98. The cutaneus quinti $\left({ }^{*}\right)$, or cutaneous branch of the trigeminal nerve (§ I93), seen emerging from between the muscles on the dorsal surface of the head, and passing backwards and slightly outwards immediately beneath the skin. Soon after its origin it gives off two or three small nerves which pass backwards and upwards, and, uniting with one another form a trunk $\left(V^{\prime}\right)$ which runs along the bases of the dorsal fins, as far as to the caudal. The main trunk of the cutaneus quinti divides into two branches, one of which $\left(V^{\prime \prime}\right)$ passes downwards and backwards to the anal fins, supplying them in the same way as the dorsal branch supplies the dorsal fins : the other $\left(V^{\prime \prime \prime}\right)$ passes along the outer face of the clavicle and sends branches to the pectoral and pelvic fins. Traced forwards, the cutaneus quinti is seen to make its exit from the skull by a foramen in the parietal ( $\$ 3 \mathrm{I}$ ).

99. The cutaneus vagi, or cutaneous branch of the pneumogastric nerve ( $\$ 196)$, emerging from beneath the operculum near its dorsal end, having already divided into two trunks, which pass almost directly backwards, the first $(X)$ immediately beneath the skin of the lateral line, the other $\left(X^{\prime}\right)$ along the line of junction of the dorsal and ventral muscles; at about the level of the second dorsal fin these nerves begin gradually to approach one another, and the upper one soon farles off, the lower one then passing along the lateral line to the tail. The two trunks are united by one or two commissures.

XVIII. Keeping the fish with its left side uppermost, make a median ventral incision from the hipgirdle backwards to within a quarter of an inch of 
the anus. Take a second incision passing vertically dorsalwards from the anterior end of the first, and a third sloping obliquely backwards and upwards from its posterior end: carefully lift up the flap of muscle as you make these cuts so as to be sure that none of the internal organs are injured. Extend both cuts upwards until the body cavity is well exposed: then fasten back or remove the flap of muscle. Observe the following points without further dissection :-

I00. The abdominal cavity, enclosed by the muscular walls of the body, ending in front at about the level of the shoulder girdle, and extending posteriorly for a short distance behind the anus.

IоI. The peritoneum (parietal layer), a pigmented membrane lining the abdominal cavity. The visceral layer of the peritoneum is reflected over the viscera (see $\S$ I Io).

102. The liver (Fig. 3I, Ir), a large brown-coloured organ, with its broad attached end at the anterior boundary of the abdomen and its long left $\left(l r^{-3}\right)$ and short right $\left(l r^{-}\right)$ and middle $\left(l r^{-2}\right)$ lobes extending backwards towards the posterior end of the cavity.

Io3. The stomach (Fig. 3 I, st), a wide thick-walled tube, passing from the front wall of the abdomen, backwards to within a short distance of the anus, where it becomes bent forwards upon itself: in the undisturbed position of the parts it is largely covered by the liver.

104. The intestine (Fig. $3^{1}$, dm., il., r(t), a coiled tube, connected anteriorly with the recurved portion of the stomach, and ending behind in the anus (see § I I 9 ).

105. The pyloric cæca (Fig. 3I, py.c) numerous small blind tubes, arranged in bunches around the small intestine at its junction with the stomach. 
106. The spleen, a smooth, dark red body, of elongated form, situated somewhat dorsal to, but not directly connected with the stomach.

I 07. The gall-bladder (Fig. 3 I, g.b), a large ovoid sac, filled with bright green bile, situated about the middle of the abdominal cavity towards the right side.

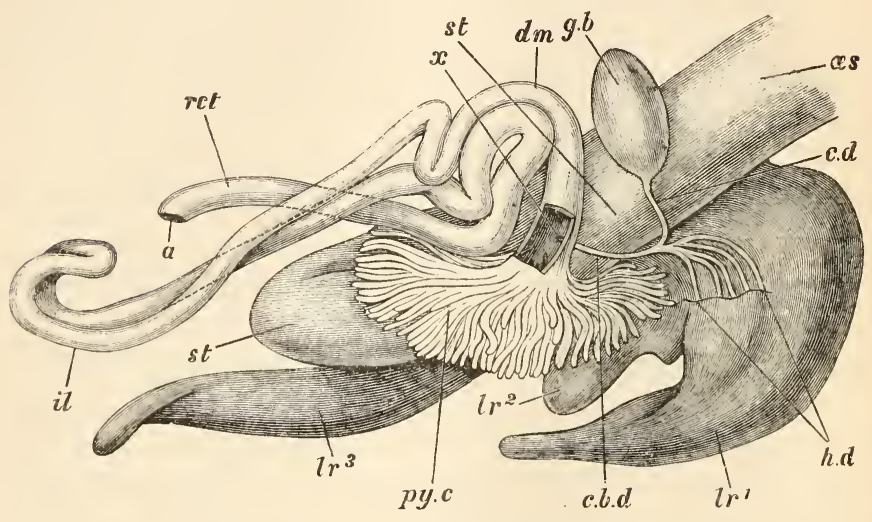

FiG. 3I.-Gadus morrhua. Dissection of the alimentary canal and its glands, from the right side ( $\left(\frac{1}{2}\right.$ nat. size).

$a$, anus : c.b.d, common bile duct : c.d, cystic duct : $d m$, c̀uodenum : $g . b$, gall-bladder : h. $d$, hepatic duct $: i l$, ileum $: l r^{1}$, right lobe of liver : $l r^{2}$, middle lobe of liver: $l r^{3}$, left lobe of liver: a's, œsophagus: fy.cc, pyloric cæca: rct, rectum: st, stomach: $x$, aperture of bile duct, below it apertures of pyloric cæca.

I08. In the male, the testes, long lobulated glands, having much the appearance of fat, and extending horizontally along a considerable proportion of the length of the abdominal cavity: their size varies greatly according to the season and the age of the fish.

ro9. In the female, the ovaries, conical sacs, of a pink 
colour, situated in the posterior part of the abdominal cavity, above the termination of the intestine.

I 10 . The visceral layer of the peritoneum, a thin transparent membrane, enveloping, somewhat loosely, the foregoing viscera.

I I I. The air-bladder or swim-bladder, corering the whole of the dorsal wall of the abdomen, the peritoneal lining of which is continued over its ventral surface: if in the distended condition, its smooth median and sacculated lateral regions render it readily distinguishable; if collapsed, it is less obvious.

I 2. The ureter, a thin-walled tube, passing upwards from the urinary aperture to the posterior end of the airbladder to join the kidney, which is at present concealed : near its ventral end it gives off a bilobed diverticulum, the urinary bladder.

XIX. Continue forwards the median rentral incision already made, by cutting through the hip-girdle and between the right and left halves of the shoulder-girdle: dissect away the left half of both pectoral and pelvic girdles, working from below upwards, and taking especial care not to cut too deeply and so run the risk of injuring the hepatic (§ I 25) and precaval ( $\$$ I 4 I) veins. Note

I 3 . The gullet (Fig. 3 I, ces), a tube of about the same diameter as the stomach, with which it is continuous posteriorly, while in front it passes into the pharynx (§ XXX.).

II4. The pericardial cavity; a small chamber containing the heart (§ I I5) and separated by a strong fibrous partition - the pericardio-peritoneal septum-from the cavity of the abdomen : it is bounded above by the floor of the mouth and bases of the gills, and below by the 
ventral ends of the clavicles and the muscies in connection with them.

I I5. The heart (Fig. 32), consisting of (a) the small, thin-walled sinus venosus $(s . v)$, attached by the hepatic veins ( $§$ I25) to the pericardio-peritoneal septum; $(b)$ the large, irregular thin-walled auricle (cuı), situated in the dorsal region of the cavity, and largely concealing $(c)$ the firm, prismoidal ventricle $(v)$, which lies in the ventral region of the pericardial cavity and passes in front into $(d)$ the white, rounded bulbus arteriosus (b.a).

I 6. The pericardium, a thin membrane lining the pericardial cavity and reflected over the heart in the same manner as the peritoneum over the abdominal viscera.

$\mathrm{XX}$. Skin the top of the head and clear away the muscles until the roof of the skull is exposed: break away the latter, bit by bit, with bone forceps or strong scissors, and observe

I 7. The brain, lying loosely in the large cranial cavity, and continuous posteriorly with the spinal cord, which passes through the neural canal of the vertebral column to the tail.

I 8 . The arachnoid fluid filling the space between the brain and the walls of the cranial cavity.

Large cod are often killed by a blow on the head; this gives rise to extensive extravasation, and the cranial cavity is found to be full of clotted blood, which adheres so closely to the brain as to require care in its removal.

XXI. If no second specimen is to be dissected, go over $\S \S \times X X V I-196$ : then cut through the spinal cord about half an inch from its junction with the brain, sever the various cranial nerves, carefully detach the olfactory lobes from their attachments (§ I 89), remove the brain, and place it in alcohol 
of about 90 per cent. If a second specimen is to be dissected, the examination of the external features of the brain may be postponed, and the organ placed in alcohol at once.

XXII. Place the fish once more on its right side, and by turning forward the lobes of the liver, and, when necessary, dissecting away the peritoneum from the viscera, blood-vessels, \&c., make out the following : _ I

I I 9. The divisions of the alimentary canal (Fig. 3 I) : the œsophagus passes insensibly into the stomach, the cardia or aperture of communication between the two being marked only by a difference of colour and texture; the stomach passes back to the hinder end of the abdomen, becomes bent upon itself towards the right side, and narrowing, becomes continuous with the small intestine, the pylorus or aperture of communication between the two being marked by the attachment of the pyloric cæca ( $p y . c)$; the first loop of the intestine is $U$-shaped and represents the duodenum $(\mathrm{dm})$; this is followed by the ileum (il), which after a

1 The injection of the blood-vessels, which is necessary for their satisfactory examination, is best performed in the following way: $(a)$ Make a small incision into the bulbus arteriosus, and insert a cannula directed forwards; from this the ventral aorta and afferent branchial arteries are injected : $(b)$ insert a second cannula into one of the branches of the me:enteric artery, e.g. that supplying the pyloric cxca, directing it forwards, or towards the proximal end of the artery; from this the dorsal aorta and efferent branchial arteries are filled : $(c)$ a third cannula is inserted into one of the factors of the portal vein, e.g. that coming from the pyloric cæca, its point being directed forwards or towards the main portal vein, which is in this way thoroughly injected : (d) a fourth canuula is inserted, pointing forwards, into the spermatic vein (\$ I 26); from this the main systemic veins are easily filled. Plaster of Paris, or some other cold injecting material, must be used; as a very slight heat causes gelatinization of the connective tissue of the bloodvessels and their consequent rupture. 
minor turn, forms a long loop enclosed in a special investment of peritoneum and extending backwards posterior to the anus; passing forwards again, the ileum forms a loop within and concentric to that of the duodenum, and is then continued insensibly into the rectum $(r c t)$, which is only marked by its somewhat greater diameter and by the dark colour of its contained frecs; the rectum passes directly backwards to the anus (a), between the ileum on the right and the stomach on the left.

I 20. The way in which the viscera are supported by folds of peritoneum: the stomach is suspended to the dorsal wall of the abdomen by a vertical fold, the mesogaster: a similar fold supporting the small intestine is the mesentery: one suspending the rectum is the mesorectum: the testes are kept in position by the mesorchium, the ovaries by the mesoarium: the anterior border of the liver is connected to the anterior wall of the abdomen by the coronary ligament and its dorsal surface is connected with the stomach by the gastro-hepatic omentum.

I 2 I. The cystic duct (Fig. 3 I, c.d) from the gall-bladder $(g . b)$, joined by several hepatic ducts $(h . d)$ from the liver : from the point of junction the common bile duct (c.b.d) passes backwards and opens into the small intestine just beyond the pylorus.

122. The portal vein (Fig. 32, p), taking blood from the other abdominal vi-cera to the liver : it is a large vein receiving factors from the stomach, intestines, spleen, and pyloric cæca, as well as a branch from the air-bladder, leaving the wall of the latter at about the junction of its anterior and middle thirds, and bringing blood from the rete mirabile $(x)$ (vide infra $\S$ 139). All these tributaries unite to form a plexus of large veins in the gastro-hepatic omentum, from which several veins go off into the substance of the liver $\left(p^{\prime}\right)$.

123. The gastric branch of the vagus (tenth cerebral) nerve, a 
large nerve entering the abdomen alongside the gullet and spreading out into a number of fine branches on the surface of the stomach.

124. The cœliac and mesenteric arteries (Fig. $32, c a, m$ ), entering the abdomen above and to the right of the gullet: the former goes almost exclusively to the pyloric cæca : the mesenteric artery supplies the stomach $(g)$, intestines $(i)$, spleen $(s p l)$, and rete mirabile $(x)$.

125. The two hepatic veins (Fig. 32, $h p$ ) proceeding, one on each side, from the anterior border of the liver, through the pericardio-peritoneal septum to the sinus venosus.

126. The spermatic vein (Fig. 32, sp.v) bringing blood from the ovary or testis, from the anterior end of which it passes directly forwards to join the precaval vein $(\S \mathrm{I} 4 \mathrm{I})$ : the spermatic artery $(s p . a)$ arises from one of the gastric branches of the mesenteric.

XXIII. Remove the liver, stomach, intestine and spleen: cut open the stomach and duodenum, wash out their contents and note

I27. The rugœ of the stomach, prominent longitudinal folds into which its mucous membrane is thrown.

I28. The pyloric valve, a circular ridge of mucous membrane forming a constriction between the stomach and intestine.

I29. 'The aperture of the bile-duct (Fig. $3 \mathrm{I}, x$ ) in the duodenum, near the pyloric valve: a bristle should be passed from it into the duct.

I30. The three or four openings of the pyloric cæca (Fig. $3 \mathbf{I}$, below $x$ ), situated in the duodenum between the pylorus and the bilary aperture.

XXIV. Make out the following points in the reproductive organs, still left in situ :-

In the Male.

I3r. The testes, two elongated bodies, each consisting of a straight tubular axis, the vas deferens, with a soft greatly lobulated body, the testis proper, attached to the whole length of one side. At about one fourth of their length from the posterior end, the two testes unite with 
one another in the middle line, and send off a short common duct, which passes, closely attached to the front wall of the ureter, to the genital aperture.

\section{In the Finale.}

I32. The ovaries, two conical bodies, united with one another posteriorly and sending off from the point of junction a short oviduct which passes downwards between the ureter and the rectum to the genital aperture.

$\mathrm{XXV}$. Remove the reproductive organs and the ureter from the body, along with a small portion of the body wall containing the urinary and genital apertures: in cutting through the ureter leave a recognisable piece of its dorsal end, as it will have to be traced to the kidney ( $§$ I 43): pin out these organs under water, and make longitudinal incisions though the ureter and the vas deferens or oviduct and ovary; note

133. A median partition dividing the dorsal end of the ureter into two tubes.

In the Male.

I34. The common genital canal, receiving the vasa deferentia, opening externally by the genital aperture, and separated by a thin partition from the ureter.

135. The numerous small apertures in the vas deferens by which it receives the secretion from the lobes of the testis.

In the Female.

I36. The large central cavity of the ovary, encroached on by irregular processes of the substance of the organ, on which the ova are developed.

I37. The cavity of the oviduct, continuous with those of the two ovaries, and opening externally by the genital aperture. 
XXVI. Open the air-bladder by a longitudinal incision along its left side, and dissect away the muscles, $\& c$., which still obscure its anterior end, taking care not to injure the precaval vein (\$ I $4 \mathrm{I}$ ); note

I38. The characters of the air-bladder: its lateral and ventral walls are very thick and tough, its dorsal wall so thin that the vertebral column, aorta ( $\$$ I 42 ), and kidneys ( $§$ I 43) can be readily seen through it; its ventro-lateral portions are deeply sacculated, and its antero-lateral angles produced each into a long coiled tube, blind at its free end ; of these the left only is seen at present, imbedded among the muscles at the anterior end of the air-bladder.

I39. The rete mirabile of the air-bladder, a large, soft, cake-like mass of rounded outline and red colour, situated within the air-bladder in contact with its ventral wall: as already seen, it receives a branch from the mesenteric artery and pours its blood into the portal vein.

I40. The anterior portion of the kidney-usually called the head-kidney - a dark-brownish red mass, lying above and in front of the anterior end of the air-bladder.

I4I. The (left) precaval vein (ductus Cuzierii) (Fig. 32, $p c)$, a large vein passing from the sinus venosus upwards to the anterior part of the kidney, crossing the gullet as it goes, and lying immediately behind the last branchial arch ; it receives the corresponding spermatic vein, and at its dorsal end is formed by the confluence of two veins, the left jugular bringing the blood from the head, and the left cardinal returning that from the trunk generally.

I 42. The dorsal aorta (Fig. 32, d.ao), a large median artery, seen through the thin dorsal wall of the air-bladder; to show it plainly, the latter should be dissected away.

The dorsal aorta is continued posteriorly as the caudal artery (c.a), which passes through the hæmal arches of the tail; before doing so it 
gives off an artery $(y)$ which passes downwards aiongside the ureter (\$ I I 2 ) to supply the bladder, anterior anal fin and body muscles of that region, and also anastomoses with the intestinal artery $(i)$ by a small branch.

143. The kidneys, consisting of two irregular longitudinal bands of soft reddish-brown tissue, lying one on eacl side of the aorta; to see them satisfactorily, the dorsal wall of the air-bladder must be dissected away; anteriorly they pass each into the so-called head-kidney already seen ( $\$$ r 40); posteriorly they unite and form a large median mass, lying immediately behind the air-bladder and enclosed in the hæmal canal of the anterior caudal vertebræ, which must be cut away to display it; it is from this hinder part of the kidney that the unpaired ureter springs.

144. The cardinal vein (Fig. 32, l.cd, r.cd), a somewhat irregular trunk, running through the substance of each kidney.

The middle part of the left cardinal is aborted for some distance, the veins from the left kidney in this region passing into the right cardinal. ${ }^{1}$ Posteriorly, the two become continuous with the caudal vein $(c . v)$, which passes through the hæmal canal, ventıal to the caudal artery. A vein $(y)$ bringing back the blood from the posterior part of the air bladder, urinary bladder, anterior anal fin and body-wall, and anastomosing with the portal, runs parallel to the corresponding artery (\$ I 42 ), ard enters the caudal vein just as it emerges from the hæmal canal.

145. The sympathetic nerve is also imbedded in the kidney.

XXVII. Dissect away the muscles, \&c. lying in front of the heart and at the bases of the gills, and bring into view

I 46. The ventral aorta (Fig. 32, v.ao), passing forwards from the bulbus arteriosus and ending abruptly at about the level of the ventral end of the first gill.

${ }^{1}$ Its anterior part is sometimes connected with the right cardinal by a transverse anastomosis in this region. 
THE COD.

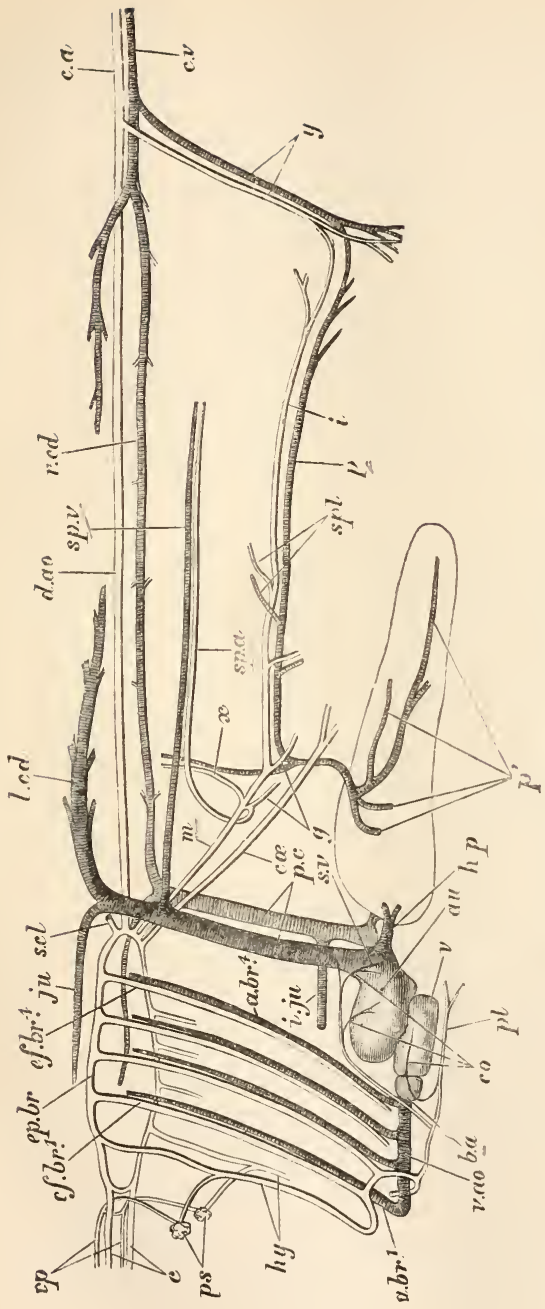

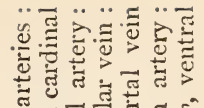
こ

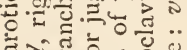
ฮี่

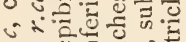

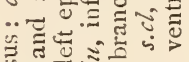

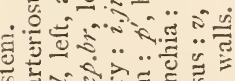

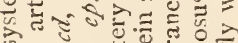

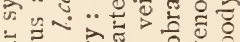

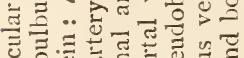

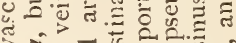
उ

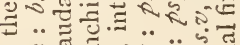
o응 ฮี

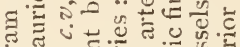
है

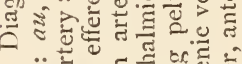

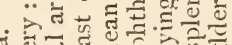

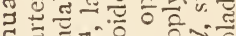
दี

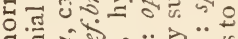
घㄷํㅇ क

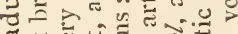

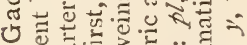
1 $\pi=>$ in ง ن 峁

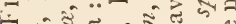
ริ

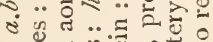

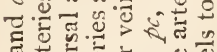
๘

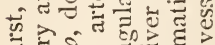

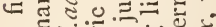
इे

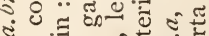
की 
The hyoidean (§ I 75) and two first efferent branchial arteries (\$ $15 \$)$ anastomose at their ventral ends, and from the junction of the two latter an artery is given off on each side, which meets with its fellow below the ventral aorta to form an azygous vessel, which is best seen at this stage. This soon divides into two branches, one of which passes above, the other below, the pericardium, supplying the muscles in these regions, and giving off several very small coronary vessels to the heart (Fig. 32, co). The ventral branch eventually divides into two, which supply the pelvic fins $(p l)$.

I 47. The afferent branchial arteries (Fig. 32, a.bri $\left.a . b r^{4}\right)$, four on each side, given off laterally from the ventral aorta to the four pairs of gills, and passing dorsalwards along the posterior face of the corresponding branchial arches.

148. The inferior jugular vein (Fig. 32, i.juc), an unpaired vein bringing the blood from the lower parts of the head : it passes through the pericardial cavity to the dorsal side of the heart, and opens into the right precaval.

XXVIII. Remove the heart and observe again the relations of its parts ( $\$$ II5); then open its various chambers by the removal of the left wall of each. This is best done under water. Observe

I49. The thin smooth walls of the sinus venosus.

I50. The sinu-auricular valve, between the sinus venosus and the auricle; it consists of two membranous flaps, respectively dorsal and ventral in position.

I5I. The thin walls of the auricle, strengthened by a network of interlacing muscular bands, the musculi pectinati.

I52. The auriculo-ventricular valve, guarding the round aperture between the auricle and the ventricle; it consists of two flaps, respectively anterior and posterior in position: 
153. The thick muscular walls of the ventricle, raised internally into a network of ridges, the columnæ carneæ.

I54. The aortic valves, consisting of two semilunar flaps situated right and left, at the junction between the ventricle and the bulbus arteriosus.

I 55. The thick fibrous walls of the bulbus arteriosus.

XXIX. Draw the gills of the left side downwards, so as to feel the muscles (levatores arcuum branchialium) connecting them with the skull on the stretch : dissect away these muscles, as well as the anterior part of the air-bladder, make out

156. The branchial branches of the vagus, distributed to the branchial arches in the same manner as in the Skate (p. $82, \S 214$ ). The origin of the gastric, cardiac, and cutaneous branches of the vagus may Le seen at the same time, as well as its exit from the skull (see $\$ 196$ ).

I57. The glossopharyngeal, dividing, soon after its exit from the skull, into two branches, a posterior going to the anterior face of the first branchial arch, and an anterior passing at first forwards, and then downwards, along the inner face of the hyomandibular to the pseudobranch (\$ I 96).

I58. The left epibranchial artery (Fig. 32, ep.br), lying longitudinally along the dorsal ends of the gill arches, and receiving the four efferent branchial arteries $\left(e f . b r .^{1}, e f . b r .{ }^{4}\right)$, one from each arch. After receiving the last efferent artery the epibranchial trunk passes inwards and backwards, and unites with its fellow of the opposite side to form the dorsal aorta, but just before doing so, it gives off a subclavian artery (s.cl) on each side, which supplies the pectoral fin. The cœliac $(c \infty)$ and mesenteric ( $m$ ) arteries ( $\mathrm{I}_{24}$ ) arise from the right epibranchial just anterior to the subclavian.

XXX. Cut through the lower jaw, the hyoid, and the branchial arches, a little to the left of the middle ventral line, so as to leave the tongue intact on 
the right side; place the fish in the supine position (dorsal surface downwards) and fasten out the mandible, hyoid, and gill arches right and left, so as to get a good view of the interior of the mouth. Observe the following:-

I 59. The numerous small premaxillary teeth arranged in two symmetrical sets on the premaxillæ.

I60. The larger mandibular teeth on the lower jaw.

I6I. The median crescentic group of vomerine teeth, a short distance behind the premaxillary teeth.

162. The superior pharyngeal teeth, arranged in four groups, two on each side, on the ankylosed pharyngobranchials ( $\$ 57)$.

163. The inferior pharyngeal teeth, one group on each of the small fifth branchial arches (\$ 62).

I 64. The small, blunt, non-protrusible tongue, supported by the median ventral portion of the hyoid arch.

I65. The absence of posterior nares or spiracles.

166. The branchial clefts, vertical fissures between the gill arches, five in number, the first being between the hyoid and first branchial arches, the last between the fourth and fifth branchials.

I67. The gill-rakers, horny filaments acting as strainers, attached to the branchial arches, and bounding the margins of the clefts.

I 68. The greatly constricted aperture of the œsophagus.

I69. The pseudobranchia has been already noticed ( $\$ 90)$ : on removing the mucous membrane, it is seen to be a rounded vascular body, resembling the rete mirabile in the air-bladder.

XXXI. Remove a portion of one of the gills on the left side, and make out by dissection and transverse sections 
I 7 O. The bony branchial arch, supporting the gill along its whole length, and having a crescentic transverse section.

I 7 I. The form and arrangement of the branchial filaments: each has the form of a right-angled triangle with a very narrow base attached to the branchial arch, and with the apex free: the hypotenuses face opposite ways in successive filaments, so that while the bases of the filaments form a single row, their free ends form a double row, each row corresponding with a demibranch in the skate (p. $46, \S 84$ ).

I72. The complete freedom of the branchial filaments owing to the absence of partitions, such as are found in the Skate (p. $45, \S 83$, and p. $69, \S \S 164,165$ ).

I 73. The afferent and efferent branchial arteries, running parallel with one another along the outer side of the branchial arch, the afferent artery being external: the branches of the afferent artery go to the inner sides (hypotenuses, $\S$ I 7 I ) of the gill filaments, the feeders of the efferent artery lie along their outer sides.

XXXII. Keeping the fish in the supine position, dissect away the pharyngobranchials of both sides so as to expose both epibranchial arteries; trace forward the latter, and dissect away enough of the mucous membrane to make out the following :

174. The carotid arteries (Fig. 32, c), continuing forwards the epibranchial trunks, and uniting with one another above the parasphenoid by a short, slender, transverse trunk, thus completing the circulus cephalicus.

I75. The hyoidean artery (Fig. 32, hy), springing from the ventral end of the first afferent branchial, and dividing into two branches, one of which forms the afferent artery of the pseudobranch $(p s)$, while the other anastomoses with the epibranchial (ep.br).

The blood is collected from the pseudobranch by an efferent (ophthalmic) artery $(o p)$, which, after curving over the corresponding carotid, and anastomosing with its fellow of the opposite side by a short transverse trunk immediately in front of the carotid anastomosis, passes to the choroid gland of the eye ( $\$ 78)$. 
176. A nerve rumning alongside the hyoidean artery is the posterior or hyomandibular division of the seventh nerve or portio dura. (See § 193.)

XXXIII. Remove the layer of transparent integument covering the exposed surface of the eye; cut away the supraorbital process of the frontal with bone forceps, and, if time permit, dissect out the ocular muscles and their nerves, which have the same essential disposition as in the skate (pp. 77,78 , §§ 191 -203) ; then remove the eye, dissect away the muscles, \&c. still attached to it, and divide it into inner and outer hemispheres by an equatorial incision, passing midway between the pupil and the entrance of the optic nerve. Note the following :-

177. The cornea, iris, lens, sclerotic, retina, and aqueous and vitreous humours, have the usual relations (pp. $8_{4}, 8_{5}, \$ \S 224-228$ ).

178. The choroid, consisting of three distinct layers; a black pig. mentary layer next the retina, a shining silvery layer next the sclerotic and between these a red vascular layer, which undergoes a great thickening around the entrance of the optic nerve, forming a sort of annular cushion; this thickening is the so-called choroid gland.

179. A slit in the retina extending from the blind sfot or entrance of the optic nerve, along the posterior (outer) side of the eyeball to the ora serrata or outer boundary of the retina; through it extend a delicate grey fold of the choroid, the processus falciformis, which ends against the posterior side of the lens in a pyriform enlargement, the campanula Halleri.

XXXIV. The fifth nerve may now conveniently be dissected (\$ I93); afterwards remove the skin in the neighbourhood of the nostrils, and observe

I8o. The small nasal sac, communicating with the exterior by the two apertures already seen $(\$ 86)$, but having no connection with the mouth: its wall is formed by the delicate, plaited Schneiderian membrane.

XXXV. Carefully break away the roof of the auditory capsule so as to expose the oryan of hearing; or, better, remove the entire auditory capsule, fasten it out firmly under water in a dissecting-dish and dissect it from the inner or cranial side. Note

I 8 I. The irregular system of cavities excavated in the bone and cartilage of the auditory capsule for the membranous labyrinth; the separation of these cavities from the brain cavity by membrane only.

182. The membranous labyrinth (Fig. 33), consisting of the ovoidal 
vestibule $(\tau)$ and of the three semicircular canals : the latter have the usual arrangement; the anterior (a.s.c) and posterior (p.s.c) canals have their adjacent limbs confluent, so that the two canals open by three apertures into the restibule; the horizontal canal (h.s.c) lies entirely to the outer side of the vestibule, into which it opens by two separate aper. tures, and the ampulles $(a)$ are situated at the actual extremities of the canals, those of the anterior and horizontal canals being anterior, that of the posterior canal posterior.

I 83 . The two otoliths, one large $(s)$ and shaped something like a shell (sagitta), and the other very smail (as) (asteriscus) lying in a small diverticulum of the vestibule, just beneath the ampulla of the posterior canal.

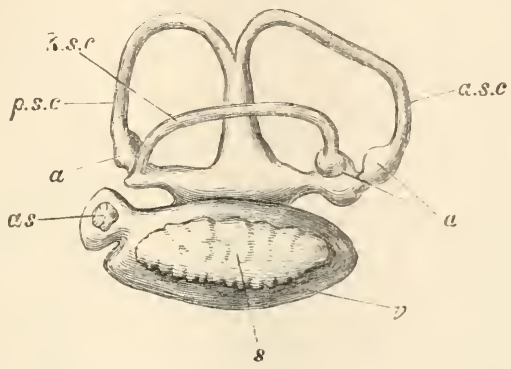

FIG. 33.-Gadus morrhua. The organ of hearing. (From a slietch by Mr. G. B. Howes.)

$v$, vestibule: a.s.c, anterior, f.s.c, posterior, and h s.c, horizontal, semicircular canals: $a, a, a$, ampullae : $s$, sagitta : as, asteriscus.

XXXVI. Examine the brain: if a fresh fish is used for the purpose and no spirit-specimen is available, the verification of the characters of the ventral surface must be left until the origins of the nerves have been made out, when the brain may be removed : note the following :-

I84. The medulla oblongata (Fig. 34, m.o), or hindmost division of the brain, continuous with, and of scarcely greater diameter than, the spinal cord. 
Its dorsal surface is marked with an obscure longitudinal furrow, continuous with the dorsal fissure of the cord, and separating two longitudinal elevations, the posterior pyramids, external to which are two sinilar elevations, the restiform bodies.

I 85 . The cerebellum $(c r b)$, a large tongue-shaped body, lying on the dorsal side of the medulla oblongata, and largely concealing it in a view from above. 'The medulla and cerebellum together form the hind-brain.

I 86. The mid-brain, consisting on the dorsal side of two considerable ovoidal masses, the optic lobes (o.l), and below of a mass corresponding to the crura cerebri (c.c), of other vertebrate brains.

I87. The fore-brain, consisting mainly of the two cerebral hemispheres $(c . h)$, irregular rounded masses, situated just anterior to the optic lobes, and of considerably smaller size than the latter: each hemisphere is marked dorsally with a furrow or sulcus parallel to its inner edge. A small somewhat rhomboidal area in the middle line, between the hemispheres and optic lobes, is all that appears externally of the 'twixt-brain or thalamencephalon, above; below it is constituted by two somewhat bean-shaped bodies, the lobi inferiores (l.i), and the infundibulum (in), lying between them (see $\$ 205$ ). On slightly separating the hemispheres they are seen to be united by a delicate transverse commissure $(\mathrm{cm})$ passing between their inner faces.

I 88. The pituitary body $(p t)$, a rounded vascular mass, of deep red colour, situated in the middle ventral line immediately beneath the thalamencephalon.

I 89. The olfactory lobes (olf), two rounded bodies, each about two-thirds the diameter of one of the cerebral hemispheres, situated in close contact with the hinder walls of the nasal sacs, and connected by delicate nerve-like cords (I) with the corresponding hemispheres: the connecting 


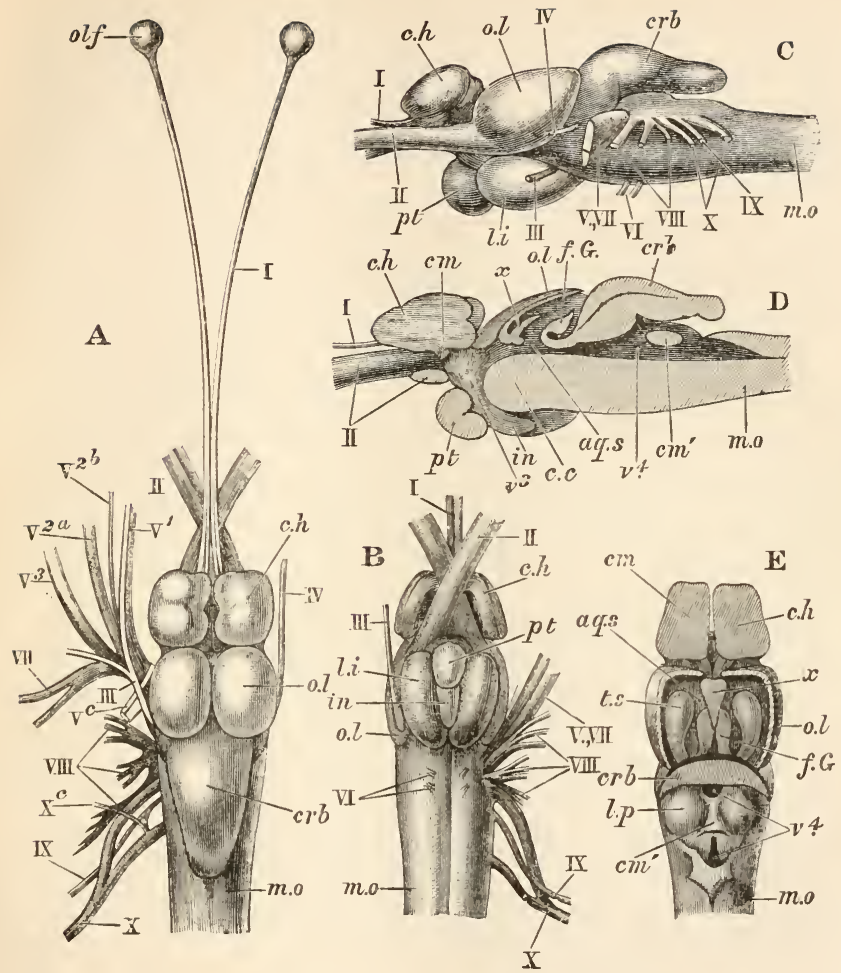

FIG. 34.-Gadus morrhua. The brain, $A$, from above ; $B$, from beneath; $C$, from the left side; $D$, longitudinal vertical section; $E$, with the ventricles laid open from above, by the removal of the greater part of the cerebellum $(c r b)$ and of the optic lobes $(0 . l)$; the cerebral hemispheres are also cut through horizontally to show the absence of lateral ventricles, and the commissure $(\mathrm{cm})$ by which the hemispheres are united (nat. size).

$a q . s$, aqueduct of Sylvius; c.c, crura cerebri : c.h, cerebral hemispheres: $\mathrm{cm}$, commissure between hemispheres: $\mathrm{cm}^{\prime}$, commissure between lobi posteriores : $c r b$, cer ebellum: f.G, fornix of Gottsche : in, infundibulum : l.i, lobi inferiores: l.p, lobi posteriores: $m . o$, medulla oblongata : 0.1 , optic lobes : $c l f$, olfactory lobes : $p t$, pituitary body: 
t.s, tori semicirculares: $v .3$, third ventricle: $v .4$, fourth ventricle : $x$, valve-like flap covering anterior part of aqueduct of Sylvius: $I$, peduncles of olfactory lobes: $I I$, optic nerve : $I I I$, third nerve: $I V$, fourth nerve: $V$, fifth nerve: $V^{1}$, orbitonasal nerve: $V^{2 a}$, maxillary nerve: $V^{2 \mathrm{~b}}$, palatonasal nerve: $V^{3}$, mandibular nerve : $V^{\mathrm{c}}$, cutaneus quinti : $V I$, sixth nerve : $V I I$, seventh nerve: $V I I I$, auditory nerve : $I X$, glossopharyngeal ; $X$, vagus: $X^{c}$, commissure between tifth and tenth nerves.

cords are single in the anterior, double in the posterior part of their course.

I90. The large strap-like optic nerves (II), arising from the ventral surface of the brain, immediately in front of the optic lobes : they pass at first directly forwards, then cross one another, the right nerve going to the left eye, and vice vers $\hat{a}$ : they make their exit from the cranial cavity through foramina in the membranous side-walls of the skull.

I9r. The third pair of nerves (oculomotor, III), arising from the ventral surface of the mid-brain, external to the lobi inferiores and passing to their foramina in the membranous wall of the skull some distance posterior to the optic foramina.

192. The delicate fourth pair of nerves (pathetic, IV), arising from the dorsal surface of the brain, between the optic lobes and the cerebellum, and leaving the skull a little above the optic foramen.

193. 'The fifth (trigeminal, V) and seventh (facial or portio dura, VII) nerves, arising together from the anterior part of each side of the medulla oblongata, and leaving the cranial cavity by the trigeminal notch in the prootic $(\S 27)$.

The fifth nerve early divides into the three characteristic divisions : the first of these (orbitonasal, $V^{1}$ ) passes forwards and upwards along the inner wall of the orbit, over the optic nerve, and supplies the parts about the snout : the second division (maxillary, $\mathrm{V}^{2 a}$ ) passes outwards 
and forwards, the greater part of it going to the upper jaw ; a branch given off near its origin (palatonasal, V-b) goes directly forwards, parallel with and close to the parasphenoid : the third division (mandibular, V3), passes forwards, downwards, and outwards to the lower jaw, upon reaching which it divides into two chief branches, one passing above, the other below Meckel's cartilage. The cutaneus quinti (Vc) arises from the main trunk. The seventh nerve takes a direction outwards, downwards, and backwards, and soon divides into two branches which pass through the two foramina in the hyomandibular (\$ 40): the anterior of these divisions soon divides again, the hindermost branch going to the operculum, the other (chorda tympani) to the lower jaw : the posterior division takes a curve inwards, backwards, and downwards, reaches the inner side of the epihyal, and passes along the inner face of the hyoidean arch.

194. The sixth pair of nerves (abducent, VI), each arising by two delicate roots from the ventral surface of the medulla oblongata.

195. The eighth pair of nerves (auditory, VIII), each arising by three large roots from the lateral surface of the medulla ; these pass directly outwards to the auditory organ.

196. The ninth (glossopharyngeal, IX) and tenth (vagus or pneumogastric, $\mathrm{X}$ ) nerves, arising together from the lateral surface of the medulla; the vagus arises by two large roots which pass outwards and backwards and join one another just before leaving the cranial cavity; the glossopharyngeal arises by one main root, a little posterior to the anterior root of the tenth, to the ventral side of which it passes to make its exit from the skull.

On leaving the skull, the glossopharyngeal divides into two branches, the anterior and smaller of which goes to the pseudobranchia, the posterior to the anterior face of the first branchial arch.

The vagus, on leaving the sliull, sends off branchial nerves which supply the branchial arches in the same manner as in the skate (p. $8 \mathrm{I}$, $\S 2$ I4), it then divides into two trunks, one passing ventralwards and 
supplying the heart and stomach, the other becoming the lateral or cutaneous nerve, the distribution of $\mathrm{w}$ hich has been already seen ( $\$ 99)$. The anterior root of the vagus gives off a small nerve which joins the cutaneus quinti $\left(\mathrm{X}^{\mathrm{c}}\right)$.

XXXVII. Lift up the cerebellum and cut it away close to its attachment; note

I97. The dorsal surface of the medulla oblongata, and the cavity (fourth ventricle, $v .4$ ) inclosed between it and the cerebellum.

Ig. The lobi posteriores (l.p), a pair of rounded elevations on the anterior part of the floor of the fourth ventricle, and between them a large transverse commissure $\left(\mathrm{cm}^{\prime}\right)$ passing from one side of the medulla to the other, and in a dorsal view giving the fourth ventricle the appearance of two distinct cavities; that these are continuous may be seen by passing a guarded bristle beneath the commissure.

XXXVIII. Carefully lift up the posterior edges of the optic lobes and remove enough of them to display thoroughly the cavity thus laid bare : observe

199. The optic ventricles, large cavities in the optir. lobes, continuous with one another in the middle line so as to form in reality a single cavity: the roof of this, just removed, is very thin, its floor extremely thick.

200. The tori semicirculares (t.s), two somewhat kidney-shaped elevations, situated, one on each side on the floor (crus cerebri) (c.c) of the optic ventricle.

201. The fornix of Gottsche $(f . G)$, a somewhat shieldshaped mass, situated between the tori semicirculares, and formed as an infolding of the posterior wall of the optic lobes.

202. A valve-like plate of nervous matter $(x)$, formed as an infolding of the anterior wall of the optic lobes, and overlapping the anterior end of the fornix of Gottsche. 
203. The aqueduct of Sylvius (aq.s) a median passage continuous behind with the fourth ventricle and covered by the furnix of Gottsche; it is best made out by passing a guarded bristle forwards from the fourth ventricle and then removing the fornix of Gottsche; it is in free communication with the optic ventricle. The anterior end of the aqueduct of Sylvius is all that represents the third ventricle $(\tau, 3)$.

XXXIX. Make a longitudinal vertical section of another brain, and observe

204. The relations of the parts already seen, particularly those of the fourth ventricle, aqueduct of Sylvius, and optic ventricle.

205. The downward continuation of the rudimentary third ventricle in front of the anterior truncated termination of the crura cerebri ; in this way the infundibulum (in) is formed ; it extends backwards and downwards between the lobi interiores, and to its anterior and inferior wall the pituitary body is attached. 
THE LIZARD. ${ }^{1}$

\section{The Green Lizard (Lacerta viridis). \\ The Sand Lizard (L. agilis). \\ The Scaly Lizard (Zootoca vivipara).}

\section{(A).-THE SKELETON.}

I. The skeleton of the lizard may be prepared either by maceration, i.e. by allowing the roughly cleaned bones to soak in water until the remaining tissues are decayed, or by plunging for a few seconds into boiling water: in either case, the muscle, connective tissue, \&c., still adhering, is afterwards dissected off until the bones are clean. It is advantageous to have two skeletons: in one the bones, with the exception of the skull, are not separated from one another, and the whole skeleton is set out and dried in the natural position: the skull should be removable, and it is as

1 The following description applies strictly to the Green Lizard, which although not an actual British species, is very common in Jersey; it is larger than the indigenous forms, and is readily obtained of the dealers in natural history objects. The distinguishing characters of the three lizards will be found on p. $156, \S 124$. 
well to make a longitudinal vertical section of it with a fret-saw. In the other skeleton the bones should be disarticulated, the vertebræ being strung on a string or wire, as separated, so as not to disturb their order, and the other bones placed out in proper position on a card: the skull should be boiled for $\frac{1}{4}$ to $\frac{1}{2}$ an hour, and its constituent bones gently pulled apart. This second skeleton will of course bear more prolonged maceration or boiling than the first or " natural " skeleton. In both cases the hyoidean apparatus ( $\$ 79-8_{3}$ Figs. 39-40), must be dissected out with great care, as it partly consists of delicate cartilages: considerable care is also required in cleaning the sternal ribs $(\$ 23)$. For the study of the chondrocranium a specially prepared skull is necessary: (see $\$$ VI. p. I 43.)

II. Observe the general composition of the skeleton as follows :

I. The vertebral column, consisting of numerous separate vertebræ, divisible into $(a)$ a cervical region of eight vertebræ, forming the skeleton of the neck; $(b)$ a thoracico-lumbar region of twenty-two vertebræ, forming, with the ribs and sternum, the skeleton of the trunk: (c) a sacral region of two vertebræ, giving attachment to the pelvis : and (d) a caudal region, consisting of a variable number of vertebræ supporting the tail.

2. The skull, articulated to the first cervical vertebra, and consisting of $(a)$ the brain-case and upper-jaw: (b) lower-jaw, composed of two separate rami : and (c) the hyoidean apparatus supporting the tongue.

3. The ribs, springing in pairs from many of the vertebræ: they become marked in the posterior cervical region, attain 
their maximum size in the anterior thoracic region, where they are united to the sternum $(\$ 4)$, and undergo a gradual reduction in size in the posterior thoracico-lumbar region.

4. The sternum and shoulder-girdle, together forming an inverted arch of mingled bone and cartilage in the anterior thoracic region, to the vertebræ of which the sternum is united by ribs.

5. The fore-limb, articulated to the shoulder-girdle.

6. The pelvis or hip-girdle, attached to the sacral vertebræ.

7. The hind-limbs, articulated to the pelvis.

III. Observe the following points in the vertebral column :

8. The characters of one of the anterior thoracic vertebræ : it consists of a ventral cylindrical portion, the centrum, concave in front, convex behind (procœlous), from which rises up on each side a vertical plate, the neural process or neurapophysis: this, uniting with its fellow in the middle dorsal line, incloses the neural arch, which is produced at its apex into a short, backwardly directed neural spine.

9. The zygapophyses, horizontal processes, two anterior, given off one from each side of the anterior border of the neural arch, and two posterior, similarly related to its posterior border: the anterior pair bear smooth articular facets on their dorsal surfaces, the posterior pair on their ventral surfaces.

Io. The capitular facets, one on each side of the vertebræ, at the junction of the centrum and neural arch and close to the anterior border of the latter : they serve for the articulation of ribs.

II. The mode of articulation of successive vertebræ: each fits by the concave anterior face of its centrum against 
the convex posterior face of the centrum next in front, while its anterior zygapophyses are overlapped by the posterior zygapophyses of the preceding vertebra.

I 2. The intervertebral foramina, spaces between the neural arches of successive vertebræ, due to the fact that each neurapophysis is notched, slightly in front, more deeply behind.

I3. The cervical vertebræ, with the exception of the first two, resemble the thoracic in essential respects, but are proportionally shorter from before backwards.

I4. A sub-vertebral wedge-bone (autogenous hypapophysis) is developed in connection with the ventral face of the centrum of the third cervical vertebra at its anterior end : it forms a small downwardly directed process, its anterior face furnishing part of the concave anterior surface of the vertebra.

15. The second cervical vertebra or axis, distinguished by the short conical odontoid process projecting forwards from the anterior face of its centrum, and constituting a separate ossification or os odontoideum. The axis, like the succeeding vertebra, has a sub-vertebral wedge-bone.

I6. The atlas, or first cervical vertebra, a ring-like bone, consisting of three separate ossifications, one ventral, representing part of the centrum, the others dorso-lateral. representing separate neurapophyses: between the latter stretches, in the fresh state, a transverse horizontal ligament, the space above which represents the neural arch of the atlas, while between it and the rentral ossification fits the odontoid bone, representing the dorsal portion of the centrum of the atlas. The anterior face of the atlas presents, ventrally, a smooth articular facet for the occipital condyle of the skull $(\S 3 \mathrm{I})$.

I7. The posterior thoracico-lumbar vertebræ differ but 
little from the anterior or true thoracic, save for the fact that their centra are somewhat shorter.

18. The sacral vertebræ, distinguished by their short centra, and by the presence of large, expanded, outwardly directed transverse processes, against which the ilia ( $\S$ IOI) abut: these processes are formed as distinct ossifications, and represent sacral ribs. The curvature of the posterior face of the centrum of the first, and of the anterior face of the centrum of the second sacral vertebræ, is somewhat less marked than in the other vertebræ: the posterior convexity of the second sacral is more than usually pronounced.

I9. The anterior caudal vertebræ resemble the sacral, but have longer centra, slenderer transverse processes, and longer neural spines.

20. The chevron bones, attached to the ventral faces of the centra of many of the anterior caudal vertebræ: each is Y-shaped, the paired upper limbs of the $\mathrm{Y}$ articulating with facets immediately beneath the posterior convexity of the centrum, while the stem or lower-limb is free and takes a direction downwards and backwards.

$2 \mathrm{x}$. The posterior caudal vertebræ undergo a gradua! simplification of structure towards the distal end of the tail, being finally reduced to small rod-like centra.

In all the caudal vertebra but a few of the anterior ones, there is a transverse vertical zone of the centrum which remains unossified, and along which the vertebra readily separates: many lizards lose their tails by the parting of the tissues across one of these weak places, and a new tail is formed, the supporting axis of which is not divided into vertebræ, but consists of an unjointed calcified rod.

IV. Make out the characters of the ribs and sternum: as follows:-

22. The sternum, (Fig. 39, p. 157, st), a rhomboidal plate 
of cartilage, with a small central fontanelle, and produced posteriorly into two slender flattened cornua $\left(s t^{\prime}\right)$ : with the antero-lateral edges of the sternum the shoulder-girdle articulates ( $(84)$, to its postero-lateral edges and cornua the sternal ribs are united ( $\$ 23)$.

23. The five anterior thoracic ribs : each consists of a dorsal bony portion (vertebral rib) articulated to the corresponding vertebra by an undivided head, and of a ventral cartilaginous portion (sternal rib) attached to the sternum. The first three sternal ribs are united with the postero-lateral edges of the sternum, the fourth and fifth with its cornua (see Fig. 39).

24. The posterior thoracic ribs do not meet the sternum, and undergo a gradual diminution in size from before backwards, the sternal rib being finally reduced to a small cartilaginous tip to the vertebral rib (see Fig. 39).

25. The cervical ribs, occurring in connection with all but the first three cervical vertebræ: those belonging to the fourth and fifth vertebræ are short and flattened, the othcrs resemble the thoracic ribs, but do not reach the sternum.

V. In the skull, note-

26. Its general shape : it has a somewhat pyramidal form, the base of the pyramid being represented by the hinder surface, the apex by the anterior extremity.

27. Its dorsal surface is formed by a large number of roofing bones, in which it is often difficult to distinguish the sutures or lines of junction between the bones from the depressions marking the insertions of the overlying epidermic scales.

28. The roofing bones are continued over the lateral surfaces of the skull, bounding the orbits or cavities for the eyes, and joining with the bones which constitute the alveolar margin along which the teeth are ranged. 
29. On either side of the anterior extremity of the skull is an aperture, the anterior nasal fossa, in which, in the undried skull, are seen the cartilages bounding the external nares (\$70, Fig. 37).

30. The foramen magnum (Fig. 37, f.m), a median rounded aperture on the hinder surface of the skull, leading into the brain cavity.

3r. The occipital condyle (Figs. 35 and 37, o.c), a rounded surface beneath the foramen magnum, articulating with the atlas.

32. The posterior temporal fossæ, two large vacuities, one on either side of and above the foramen magnum : they are bounded above and on the outside by the roofing bones, and on the inner side by the bones which limit the foramen magnum and form the actual roof of the brain cavity.

33. The parotic process (Fig. 37, p.oc), a horizontal bar of bone, extending on each side directly outwards from the side wall of the brain-case and bounding the posterior temporal fossa below : the part of the wall of the brain-case from which it springs lodges the organ of hearing, and is the auditory capsule.

34. The posterior nasal fossæ, small paired apertures on the ventral surface of the snout, at about the level of the front edge of the orbit.

35. The palatine foramen, a large oral aperture, on each side, just behind the posterior nasal fossa, and internal to the hindermost teeth.

36. The inferior temporal fossa, a large vacuity, separated by a bony bar (the transpalatine, $\S 6$ I) from the palatine foramen, and bounded externally by a number of small scale-like bones which continue backwards the alveolar margin.

37. The glenoid surface, for the articulation of the 
lower jaw; a pulley-shaped surface, forming the hinder angle of the inferior temporal fossa, and borne by a curved bone, the quadrate ( $\$ 53)$.

38. The basis cranii, a broad plate forming the floor of the brain-case, and continued forwards in the perfect skull by the interorbital septum (Figs. 35 and $3 i$, i.o.s), a plate of cartilage forming a median vertical partition between the orbits $(\$ 69)$.

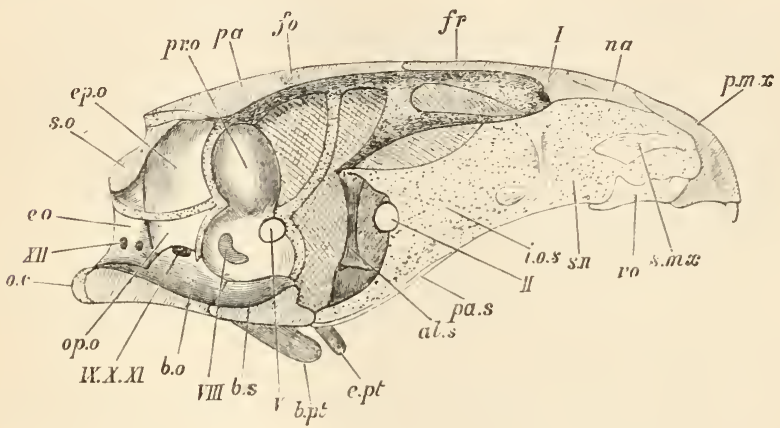

Fif. 35.-Lacerta agilis. Longitudinal ve tical section of the skull (after W. K. Parker, slightly altered) $\times 4$. The cartilaginous parts are distinguished by dotting, the membranous spaces hy crosshatching.

als, alisphenoid : b.o, basinccipital : b.pt, basipterygoid : b.s, basisphenoid : e.o, exoccipital : ep.o, epiotic ; e.pt, epipterygoid : fo, fontanelle : fr. frontal : i.o.s., interorbital septum : na, nasal : o.c, occipital condyle : $o p . o$, opisthotic : $p a$, parietal : pa.s, parasphenoid : $\hat{f} \cdot m x$, p-emaxilla : pr.o, prootic: s.mx, septo-maxillary: s.n, septum nasi : s.o, supraoccipital: vo, vomer. I-XII, foramina for exit of the cerebral nerves.

39. The tympano-eustachian fossa, a depression just external to the basis cranii and bounded by the quadrate: against its dorsal wall lies a small rod of bone, the columella auris $\left(\S 6_{5}\right.$, Fig. 36$)$. 
40. The parietals (Fig. $35, p a$ ), flat paired bones, fused together in the middle line, and forming the posterior median portion of the skull roof: they are perforated in the middle line by a small aperture or fontanelle $\left(f_{0}\right)$ : each parietal sends off from its postero-external angle a process which passes outwards, downwards, backwards, and comes in contact with the outer end of the parotic process.

4I. The frontals $(f r)$, immediately in front of the parietals, to which they are articulated by a straight transverse coronal suture: anteriorly they terminate at about the level of the front border of the orbit: they are separated from one another by the delicate median frontal suture : in front each frontal gives off a descending process, which, passing vertically downwards, forms part of the anterior wall of the orbit.

42. The nasals (na), paired bones extending from the anterior border of the frontals to the posterior border of the anterior nasal fossa.

43. The premaxilla $(p \cdot m x)$, a median bone forming the extremity of the snout : it consists of an alveolar portion bearing the four anterior teeth of each side, and of a nasal process, which extends upwards, between the anterior nasal fossæ, to meet the nasals.

44. 'The maxillæ, paired bones, continued backwards from the outer ends of the premaxilla along the sides of the skull : each consists of an alveolar portion bearing the remainder of the teeth, a narrow palatine plate, extending horizontally inwards from the bases of the teeth, and an ascending process, which extends upwards between the orbit and the anterior nasal fossa, articulating with the nasal and prefrontal $(\$ 48)$, and forming the lateral wall of the snout.

45. The jugals, paired bones, each articulated to the 
corresponding maxilla, and forming the posterior half of the ventral boundary of the orbit, and the ventral half of its posterior boundary : a process of it extends forwards, within the maxilla, almost to the anterior boundary of the orbit: its posterior inferior region is produced into a blunt, backwardly-directed spur (see $\$ 47$ ).

46. The postorbitals, roofing bones, articulating one with the outer border of each parietal.

47. Between the outer border of the postorbital, the anterior border of the quadrate, and the posterior border of the jugal, lie a number of small, irregular, scale-like bones, which form a continuous bony sheet behind the orbit, covering the posterior portion of the jugal, to see which they must be removed.

48. The prefrontal, a small bone, intercalated on each side between the anterior lateral region of the frontal and the ascending process of the inaxilla, and extending downwards to the anterior border of the orbit.

49. The supraorbitals, two rows of small bones forming the upper boundary of each orbit: the inner row, articulating with the outer border of the frontal, and extending between the prefrontal in front and the postorbital behind, consists of four bones, of which the foremost and the hindmost are considerably smaller than the intermediate ones: the outer row consists of five small bones, the hindmost of which forms the upper part of the posterior border of the orbit, while the others bound its dorsal border.

50. The lacrymal, a small bone perforated by an aperture for the lacrymal duct, situated just within the anterior boundary of the orbit, and articulating externally with the ascending process of the maxilla.

51. The supratemporals, two bones on each side, of which the first overlaps the posterior edge of the postorbital, 
while the second is a small sickle-shaped bone, closely applied to the outer surface of the external process of the parietal.

52. 'The squamosal, a similar bone to the second or hinder post-temporal: it lies external to the latter and beneath the first post-temporal, which must be removed to show it.

53. The quadrate, a stout bone articulating dorsally with the parotic process, and furnishing at its distal end the glenoid surface for the articulation of the mandible: it is strongly curved, convex anteriorly, and hollowed out behind.

54. The basioccipital (Fig. 35, b.o), a median bone forming the posterior part of the basis cranii, and furnishing the middle third of the occipital condyle (o.c).

55. Large paired bones forming the lateral boundaries of the foramen magnum, furnishing the outer thirds of the occipital condyle, and continued outwards as the parotic processes : each consists of the ankylosed exoccipital, (e.o) (posterior portion), and opisthotic (op.o) (anterior portion).

56. A median bone completing the foramen magnum above, and continued upwards as the vertical-sagittal crest to the parietals : the median portion of this bone is the supraoccipital (s.o), its lateral portions represent the epiotics (ep.o.).

57. The prootics ( $p r . o)$, paired bones continuing forwards the combined exoccipitals and opisthotics with which they articulate posteriorly, thus forming the anterior half of the side walls of the brain-case: above they articulate with the epiotics.

58. The pro-, epi-, and opisthotics together form the auditory capsule: the cavities for the three semicircular canals can be seen through them (Fig. 37, a.s.c, ps.c. h.s.c). 
59. The basisphenoid (Fig. 35, b.s), continuing forwards the basioccipital, with which it articulates by a straight transverse suture, and thus forms the front half of the basis cranii : its lateral edges give off the outstanding basipterygoid processes $(b . p t)$, its front edge is continued into a delicate bony style, the parasphenoid (pa.s), which underlies the posterior portion of the interorbital septum.

6o. The pterygoids, elongated paired bones on the rentral surfaces of the skull, forming the inner boundaries of the inferior temporal fossæ: each articulates with the corresponding basipterygoid process, is continued backwards and outwards as a process which applies itself against the inner face of the quadrate, and is continued forwards to about the middle of the inner edge of the palatine foramen, sending off at the hinder boundary of that foramen a short, outwardly directed process.

6r. The transpalatine, or os transversum, a stout bone extending between the maxilla externally and the pterygoid internally, and forming, with the process of the latter mentioned at the end of the last paragraph, the postero-external boundary of the palatine foramen.

62. The palatine, a flat bone continuing forwards the anterior process of each pterygoid, and with it completing the inner boundary of the palatine foramen: anteriorly it sends a process outwards to the maxilla, thus completing the palatine foramen in front, and is continued forwards for a short distance, furnishing the hinder boundary of the posterior nasal fossa.

63. The vomers ( $v 0$ ), paired bones lying close to one another in the middle line, in front of the palatines, by which they are embraced posteriorly, while in front they articulate with the premaxilla and maxilla; they are strongly convex below, concave above. 
64. The epipterygoid (e.pt.) (so-called columella), a slender rod of bone, lying just in front of and external to the anterior edge of the prootic: below it articulates with the pterygoid, above with the prootic.

65. The columella auris (Fig. 36), a small rou of combined bone and cartilage, lying in the dorsal wall of the tympanic recess : its inner end is inserted into the fenestra ovalis, a small aperture between the prootic and opisthotic, while its outer end is, in the entire head, fixed to the inner surface of the tympanic membrane.

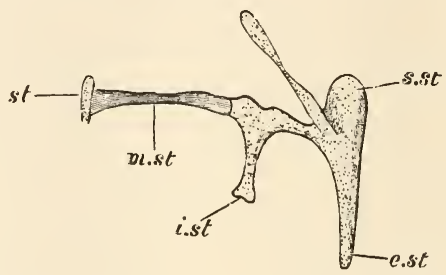

FIG. 36. - Lacerta agilis. The columella auris (after W. K. Parke:-) $\times$ I4. The cartilaginous parts are dotted.

e.st, extra-stapedial: i.st, infra-stapedial : m.st, medio-stapedial: s.st, supra-stapedial : st. stapes.

The columella auris consists of the following distinct parts which are only to be made out by careful dissection of an entire hear: $(a)$ the stapes, $(s t)$, a small cartilaginous nodule in the fenestra ovalis : (b) the medio-stapedial (m.st), a bony bar connected with the stapes, the ossification from it extending into the latter : $(c)$ a cartilaginous rod continuous with the distal end of the medio-stapedial, sending off a downwardly directed process, the infra-stapedial (i.st), and expanding at its outer or free extremity into a bar set transversely to the rest of the columella like the head of a hammer : the lower somewhat pointed end of this bar is the extra-stapedial (e.st) : its dorsal extremity, the suprastapedial (s.st) is blunt and rounded, and gives off a process which becomes connected with the auditory capsule.

66. The vagus foramen (Fig. 35 , IX, X, XI,), a small aperture in the combined exoccipital and opisthotic, behind 
and below the fenestra ovalis, and serving for the passage of the 9 th, Ioth, and IIth cerebral nerves: the I 2 th nerve makes its exit by two small condylar foramina in the posterior part of the exoccipital (XII).

67. A notch $(\mathrm{V})$ in the anterior border of the prootic, converted into a foramen in the complete skull by the membranous side-walls of the anterior moiety of the braincase $(\$ 69)$; it transmits the $5^{\text {th }}$ and 7 th nerves: behind and below it and also in the prootic is the internal auditory meatus for the exit of the 8 th nerve (VIII).

VI. The foregoing sections, with the exception of the part of $\S 65$ in small type, can be made out on the dried skull : for the following sections prepare a second skull with great care, giving special attention to the preservation of the interorbital septum : boil it in water for a few minutes, and then carefully remove the roofing bones, the premaxilla, maxilla, vomers, palatines, transpalatines, and pterygoids : note

68. By the removal of the above-mentioned membrane-bones, the primordial skull or chondrocranium (Fig. 37) is left, and is seen to consist of cartilage containing certain endogenous ossifications or cartilage bones, namely the basi-, ex-, and supraoccipital, the basisphenoid, and the pro-, epi-, and opisthotic.

69. The interorbital septum (Figs. 35, 37. i.o.s), a median vertical plate of cartilage continued forwards from the anterior border of the basisphenoid to the junction of the palatines and vomers, whence it further extends as the septum nasi $(s . n)$ to the end of the snout, forming the partition between the nasal sacs; the dorsal edge of the interorbital septum closely underlies the anterior part of the brain, and gives off, on each side, a partly cartilaginous partly membranous plate, directed upwards and outwards, which, uniting behind with the prootic, above with the skull roof, and in front with the descending processes of the frontals, furnishes a side-wall to the anterior part of the brain-case : the interorbital septum and its wings have in transverse section the form of a Y. Paired ossifications in the posterior part of the membrano-cartilaginous skull walls represent the alisphenoids ( $a l . s$ ).

7o. The nasal capsules (Fig. $37, n a$ ), rounded cartilaginous enclosures, lying one on either side of the septum nasi, of which they are lateral developments; each consists of a roof which springs from the dorsal edge 
of the septum, passes almost directly outwards, and then bending down wards, furnishes the lateral wall of the capsule, finally turning inwards towards the base of the septum to form its floor. Each capsule is per-

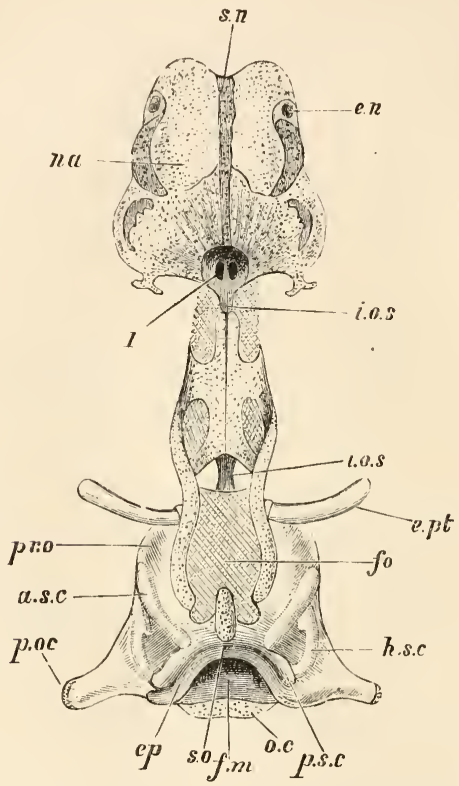

FIG. 37.-Lacerta viridis. The chondrocranium from above (after W. K. Yarker) $\times 4$. The cartilaginous parts are dotted, the membranous spaces cross-hatched.

al.s, alisphenoid : a.s.c, position of anterior semicircular canal : e.n, external nares : $e p$, epiotic : e.pt, epipterygord: $f . m$, foramen magnum: fo, fontanelle: h.s.c, position of horizontal semicircular canal : i.o.s, interorbital septum: na, nasal capsule : o.c, occipital condyle : p.oc, parotic process : pr.o, prootic: p.s.c, position of posterior semicircular canal: s.n, septum nasi : s.o, supraoccipital.

forated by the anterior nares in front, by the posterior nares below, by the foramen for the olfactory nerve behind, and above by a cres. centic slit, the olfactory fenestra. 
71. A cartilaginous process, the inferior turbinal, passes invards from the anterior part of the lateral wall of the olfactory capsule, and serves to increase the surface of the olfactory mucous membrane.

72. The septo-maxillary (Fig. 35, s.mx), a small (paired) nodule of bone, attached to the side of the nasal septum near its anterior termination.

VII. In each ramus of the mandible make out the following bones :-

73. 'The articular, forming the proximal end of the jaw, and furnishing both the cavity for articulation with the quadrate, and the backwardly produced angle or angular process of the mandible : from its anterior or distal end, which is enclosed in a sheath formed by the dentary $(\$ 76)$ the slender Meckel's cartilage is continued forwards to the sympinysis or point of junction of the two rami.

74. The angular, a splint-like bone covering the ventral edge and the lower half of the outer surface of the articular, but leaving the angular process exposed.

75. The supra-angular, similarly related to the dorsal edge and the upper half of the outer surface of the articular.

76. The dentary, forming the main part of the distal portion of the mandible and bearing all the teeth.

77. The splenial, a flat splint applied to the inner face of the dentary, and fitting, at its hinder end, into a notch between the articular and the angular.

78. The coronary, a small somewhat conical bone, forming the upwardly directed coronoid process immediately behind the last tooth: it articulates with the articular, dentary, and splenial.

VIII. The hyoid apparatus (Figs. 39 and 40) consists of the following parts:-

79. The body of the hyoid or basi-hyal (Fig. 40, b.hy'), a median flattened rod of cartilage, pointed in front.

so. The anterior cornua (a.co), paired rods of cartilage, 
extending from the basi-hyal at first outwards and forwards, and then, almost immediately, outwards and backwards, curving round the gullet, and finally coming in close contact with the ventral surface of the auditory capsule. The short ventral portion of the anterior cornu is the hypo-hyal, the next considerably expanded portion is distinguished as the cerato-hyal, the dorsal slender part is the stylo-hyal.

8I. The middle cornua (m.co), paired rods springing from the basi-hyal just posterior to the anterior cornua, to which they run more or less parallel : the proximal ends are ossified. The middle cornua represent the-first branchial arch.

82. The posterior cornua $(p . c o)$, paired cartilaginous rods, springing from the posterior edge of the basi-hyal, and passing backward and slightly outwards : they represent the hypo-branchial region of the second branchial arch (see description of the skate's branchial arches p. $38, \S 4 \mathrm{I}$ ).

83. A small curved cartilaginous rod attached by fibre to the dorsal end of each middle cornu : it probably represents the dorsal or epibranchial region of the second branchial arch.

IX. In the shoulder-girdle note

84. The coracoids (see dotted outline in Fig. 39), flat paired bones articulating with the antero-lateral edge of the sternum: each furnishes the central half of the glenoid cavity for the articulation of the humerus, and is divided by a large fenestra into an anterior bar, the precoracoid, and a posterior portion, the coracoid proper.

85. The scapulæ, articulating each with the outer extremity of the corresponding coracoid, and forming the dorsal half of the glenoid cavity.

86. The suprascapulæ, continuous with the expanded 
dorsal extremities of the scapulæ, and passing inwards towards the vertebral column : they expand gradually to the dorsal edge, and consist, like the sternum, of partly calcified cartilage, the remainder of the shoulder-girdle being formed of true bone.

87. The interclavicle, a cruciform bone, having a long posterior limb directed backwards over the ventral surface of the sternum, a short anterior limb, and long lateral limbs passing directly outward a little anterior to the level of the glenoid cavities.

88. The clavicles, flat curved bones, articulating in the middle central-line with one another, and with the anterior limb of the interclavicle, and passing at first directly outwards, then outwards and upwards to the anterior border of the suprascapula to which they are united by fibrous tissue.

$\mathrm{X}$. In the fore-limb make out the following :-

39. The general composition of the limb : it consists of a proximal division (skeleton of the brachium, $\S$ I I 7 ) containing a single bone, the humerus; a middle division (skeleton of the antebrachium), containing two bones, a smaller, internal, the radius, and a larger, external, the ulna; and a distal division the manus, consisting of a carpus or wrist, and of five digits.

90. The position of these parts : the humerus is directed from its proximal or attached end, backwards, upwards, and outwards; the ulna and radius pass, from their articulation with the humerus, downwards and slightly forwards; the manus has its digits directed forwards and outwards.

9r. If the limb is extended at right angles to the long axis of the trunk, it presents a dorsal and a ventral surface, an anterior or pre-axial and a posterior or post-axial border; the radius is then seen to be preaxial and the ulna post-axial. In the ordinary position the 
pre-axial border of the humerus is external, and in the forearm, the distal end is rotated in such a way that while the pre-axial border looks forwards and outwards at the proximal end of the fore-arm, it looks directly inwards at its distal end: the manus, being rotated with the fore-arm, has its pre-axial border inwards.

92. The humerus, an elongated bone, consisting of a shaft of true bone and of proximal and distal extremities or epiphyses of calcified cartilage. Its proximal end or head is rounded, and articulates with the glenoid cavity: on the pre-axial or radial border the shaft is produced into a large process, the radial tuberosity, on its post-axial or ulnar border into a smaller ulnar tuberosity, the latter being partly formed by the proximal epiphysis. At the distal end of the humerus is the pulley-like trochlea, divided into a smaller radial and a larger ulnar articular surface: the distal end of the shaft has its pre-axial border produced into the external or radial condyle, its post-axial border into the internal or ulnar condyle, both processes immediately proximal to the trochlea.

93. The radius, a slender bone consisting of an ossified shaft and of two calcified epiphyses : the proximal end or head is disc-shaped and slightly excavated for articulation with the humerus, the distal extremity is wide from side to side, concave for articulation with the carpus, and produced pre-axially into the radial styloid process.

94. The ulna, also formed of shaft and epiphyses; its proximal end is produced into a blunt upwardly-directed process, the olecranon, which, along with the actual proximal end of the bone, furnishes the concave sigmoid cavity, or articular surface working over the trochlea of the humerus. The distal end of the ulna is convex and articulates with the carpus. 
95. The carpus, consisting of ten small nodular bones, or rather calcified cartilages; three of these form a proximal row, the radiale, articulating with the radius, the ulnare, with the ulna, and the minute intermedium between them : five others of much smaller size form a distal row and give attachment one to each of the metacarpals $(\$ 96)$; these are called carpalia, and are distinguished by numbers, that on the radial or preaxial side being carpale $\mathbf{I}$ : a ninth bone-the centrale,--forms by itself a middle row, being intercalated between the radiale, ulnare, and second, third, and fourth carpalia : the tenth bone-the pisiform or accessory ossicle-is attached to the post-axial side of the distal epiphysis of the ulna.

96. The bones of the digits: the first or pre-axial digit (pollex) consists of three bones, a metacarpal, articulating with the first carpale, followed by two phalanges; the second of a metacarpal and three phalanges; the third of a metacarpal and four phalanges; the fourth of a metacarpal and five phalanges; and the fifth of a metacarpal and three phalanges.

97. The distal or ungual phalanges have something the form of the horny claws which they support : the remaining phalanges and the metacarpals are rod-like bones, each consisting of a bony shaft and a calcified proximal epiphysis, the distal extremity of the bone being ossified from the shaft.

XI. Observe the characters of the pelvis as follows:98. It consists of two tri-radiate bones, the ossa innominata, the dorsal limbs of which articulate with the sacrum, while the two ventral limbs unite, each with its fellow of the opposite side, in the middle line.

99. The acetabulum, a rounded concave pit, at the junction of the three limbs of the innominate bone, and serving for the articulation of the femur ( $\$$ I ro). 
roo. Each limb of the os innominatum is a separate bone, the three meeting in the acetabulum in the tri-radiate suture.

ror. The ilium or dorsal ossification of the innominate bone: it is an irregular rad, flattened from side to side, and passing from its sacral articulation downwards and forwards to the acetabulum, of which it forms the dorsal third.

ro2. The pubis or antero-ventral pelvic bone: it is somewhat flattened from above downwards, and forms the antero-ventral third of the acetabulum, whence it takes a direction downwards, forwards, and inwards to meet its fellow in the median symphysis pubis (see dotted outline in Fig. 39).

103. The prepubis, a small rhomboidal nodule of calcified cartilage, situated in the middle line between the anterior ends of the pubis.

ro4. 'The ischium, or postero-ventral pelvic bone, an irregular flattened bone, forming the postero-ventral third of the acetabulum, and passing downwards and inwards to meet its fellow of the opposite side in the symphysis ischii, a strip of calcified cartilage being interposed between the two bones.

105. The foramen cordiforme, a heart-shaped space bounded by the pubes in front, and the ischia behind : in the recent state a median ligament divides it into paired obturator foramina.

106. The os cloacæ, a small rod of bone passing horizontally backwards from the symphysis ischii in the ventral wall of the cloaca (\$ 176$)$.

XII. In the hind limb note

ro7. Its general composition : it consists of three divisions; a proximal, consisting of a single bone, the femur; a middle division or crus, consisting of two bones, a larger internal, the tibia, and a smaller, external, the fibula; and a distal 
division or pes, consisting of a tarsus or ankle, and of five digits.

ros. The position of the bones: the femur is directed from its proximal end forwards, upwards, and outwards ; the tibia and fibula pass from their articulation with the femur almost directly downwards; and the digits are directed forwards and outwards.

ro9. If the limb is extended at right angles to the long axis of the trunk, it presents, like the fore-limb ( $\oint_{9 I}$ ), lorsal and ventral surfaces, and pre- and post-axial borders : it is then found that the tibia is pre-axial, and the fibula post-axial, and that, in the ordinary position of the parts, the pre-axial border, in all three divisions of the limb, is internal.

I Io. The femur, a stout, slightly-curved bone, consisting of shaft and epiphyses, the proximal epiphysis being, however, hardly distinguishable in the adult. The proximal end of the bone is produced into a prominent ovoiàl head for articulation with the acetabulum; below and internal (preaxial) to the head is a large process representing the lesser trochanter; the post-axial greater trochanter is almost obsolete. The distal end of the bone is pulley-shaped, and forms internal (pre-axial) and external (post-axial) condyles for articulation with the tibia. Immediately above (proximal to) the external condyle is a process serving for the articulation of the fibula, and answering to the external tuberosity.

I II. The tibia, a stout, greatly-curved bone, consisting of shaft and epiphyses: along its anterior (dorsal) face runs the longitudinal cnemial ridge, which is especially pronounced at the proximal end, giving the bone a triangular transverse section: the proximal end presents two surfaces, the internal and external tuberosities, for articulation with the condyles of the femur. 
I I 2. The fibula, a slender bone also consisting of shaft and epiphyses: its proximal end or head is narrow, and articulates with the external tuberosity of the femur: its distal end is widened laterally for articulation with the calcaneum (§ II 4).

I13. Between the femur and the tibia is a thin, cartilaginous plate, incompletely divided into two portions which answer to the internal and external semi-lunar fibro-cartilages of man : each half contains two calcifications or sesamoid bones which, in the dried skeleton, are seen to be interposed two between each femoral condyle and the corresponding tibial tuberosity. A large sesamoid bone is situated to the outer side of this cartilage, and is interposed between the head of the fibula and the external tuberosity of the femur. There is also a small sesamoid attached to the ventral surface of the femur, just above (proximal to) the external condyle.

I 14. The tarsus, consisting of three bones divisible into a proximal and a distal row : the proximal row consists of a single large bone, the tibio-fibulare, representing the ankylosed tibiale or astragalus and fibulare or calcaneum ; it presents two concave articular surfaces on its proximal side for the tibia and fibula; distally it articulates by a somewhat pulley-shaped surface with the distal tarsals. Of these latter-the tarsalia-only two are separate bones in the adult; one of these (tarsale 3) being a small, calcified nodule in contact with the proximal end of the third metatarsal, the other (cuboid or tarsale 4) a larger bone giving attachment to the fourth and fifth metatarsals.

The first and second tarsalia are ankylosed in the adult with the proximal ends of the corresponding metatarsals; the fifth tarsale is absent.

I I 5. The bones of the digits correspond in number and form to those of the manus, each consisting of a metatarsal with two, three, four, five, and three phalanges respectively: 
the metatarsal of the first or pre-axial digit (hallux) and that of the second articulates with the tibial side of the tibio-fibulare, the remaining metatarsals with the tarsalia, as described above ( $§$ II 4 ).

\section{B. Directions for Dissection.}

XIII. Make out the following external characters :-

I 6 . The elongated, flat-sided trunk, separated in front by a slight constriction or neck, from the pyramidal, somewhat depressed head, and passing behind into the cylindrical tapering tail, which, in uninjured specimens, may be nearly twice as long as the head and trunk together.

I 7 . The fore-limbs, springing one on each side from the anterior end of the trunk, towards its ventral aspect: when extended at right angles to the trunk, each is seen to present a dorsal and a ventral aspect, an anterior or preaxial, and a posterior or post-axial border ; and to consist of three divisions, a proximal, the upper arm, or brachium, a middle, the fore-arm, or antebrachium, and a distal, the hand, or manus; the latter is terminated by five clawed digits, of which the first, or pre-axial, is the shortest, and is distinguished as the thumb, or pollex ; the fifth, or post-axial digit, is next in size, then comes the second, while the third and fourth are of nearly equal length (cf. $\$ \$ 89-97$ ).

I18. The hind-limbs, springing one on each side from the posterior end of the trunk, towards its ventral aspect: they present the same surfaces and borders as the fore-limbs, and are similarly divisible into three regions: a proximal, the thigh or femur; a middle, the shank or crus; and a distal the foot, or pes : the latter, like the hand, is terminated 
by five clawed digits; of these the first is the shortest, the second and fifth are of nearly equal length; the fifth, however, being the slenderer, and appearing shorter from the fact that it arises nearer the proximal extremity of the hand ; the third is considerably longer than the second, and the fourth slightly longer than the third (cf. $\$ \$ 107$ - I I 5).

I I 9. The large mouth, the gape beginning at the end of the snout and extending backwards nearly to the auditory apertures ( $\$$ I 22 ).

I20. The nostrils, or anterior nares, small, rounded apertures, situated one on each side of the snout, a short distance from its anterior end.

I2I. The eyes, situated one on each side of the head, about midway between the corresponding nostril and auditory aperture ( $\$$ I 22) : each is guarded by a short, thick upper eyelid, and a deep, thin lower eyelid, which, when the eye is closed, covers the greater part of it: within the eyelids is the thin, transparent nictitating membrane, which springs from the anterior corner of the eye and shuts backwards over the eye-ball.

I22. The auditory aperture, situated a little posterior to the extremity of the gape, and nearly as large as the exposed portion of the eye: stretched over it, a little below the general level of the head, is the thin tympanic membrane.

I23. The vent, or cloacal aperture (Fig. 39, $c l)$, a large transverse cleft at the root of the tail.

I24. The exoskeleton, consisting of a closely-fitting armour of epidermic scales : those on the dorsal surface of the trunk are small, hexagonal in outline, and indistinctly keeled; on the ventral surface they are large and are arranged in eight longitudinal series; forming the anterior edge of the 
vent is a large pre-anal plate; the scales on the tail are elongated, keeled, and arranged in definite transverse series, giving the tail a ringed appearance; on the limbs the preaxial scales are for the most part large, the post-axial smail ;
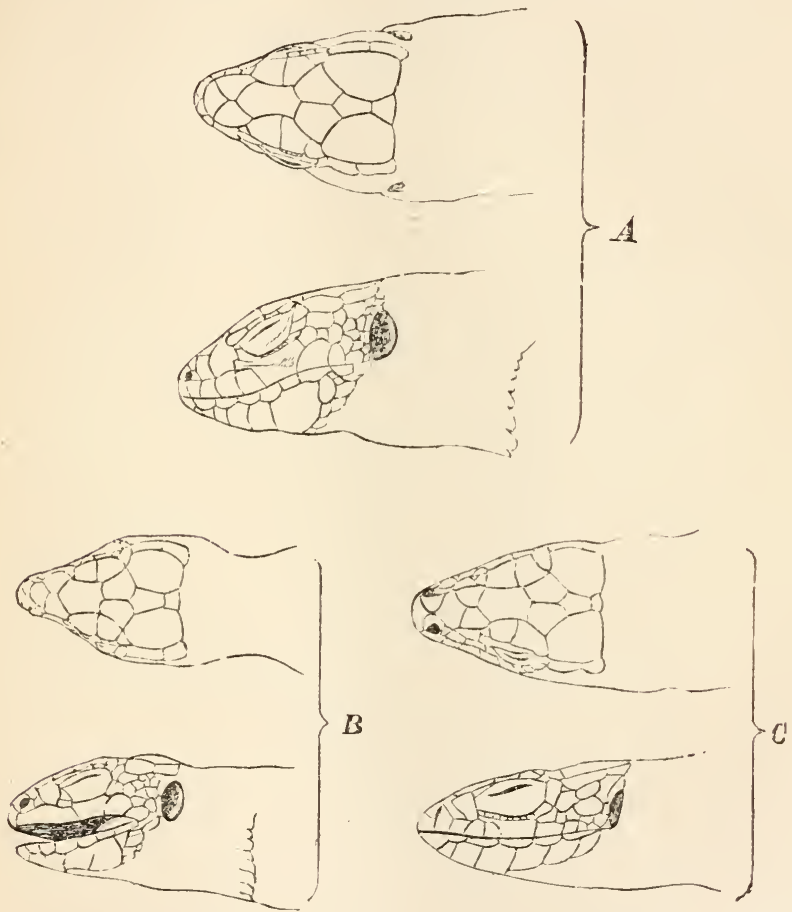

Fig. 3S. - The head from above and frcm the side of $-A$. Lacerta viridis; B. Lacerta agilis; C. Zootoca vivipara (sligbtly eniarged). After Schreiber.

on the neck they are small, except at its junction with the trunk, where there is a transverse fold bounded anteriorly by larger scales; on the head, the scales are for the most part large, and have a very definite arrangement characteristic 
of the species, many of them receiving distinctive names. Along the ventral edge of the thigh is a row of apertures (pori femorales) of a series of cutaneous glands.

The genus Lacerta is distinguished from Zootoca by the following characteristics :-Lacerta is oviparous; it has two small posterior nasal scales bounding the nostril behind, and teeth on the palate. 'Zootoca is viviparous, it has only one posterior nasal scale, and no palatine teeth.

The colouring of the three species is also characteristic. Lacerta viridis is either green or bluish-green, lighter beneath, more or less extensively marhled with black, and sometimes having either white spots, or one or two white streals on each side. Lacerta agilis is brown above with black spots, green on the sides, and white beneath. Zootoca vivipara is olive above, with a white-edged blackish streak on each side and a central black streak; the ventral surface is orange and blue (in summer) spotted with black.

XIV. Place the animal in the supine position (ventral surface upwards), and make a median incision through the skin from the chin to the anterior border of the vent : carefully separate the skin from the underlying muscles and reflect it right and left ; this is a matter requiring considerable care, especially in a fresh specimen, since some of the muscles are inserted into the ventral scales ( $§$ I 24) and are with difficulty separated from them: the rectus lateralis $(\S \mathbf{1} 25)$ is almost certain to be reflected with the skin. Note

I 25. The rectus abdominis (Fig. 39, r.i', r.l, r.i), a longitudinal paired muscle which, with its fellow of the opposite side, forms the median ventral portion of the muscular body wall. It is divided by transverse fibrous bands, or inscriptiones tendineæ, into segments, the longitudinal fibres of which are inserted into the anterior edges of the median ventral scales.

The rectus abdominis consists of three portions : a ventral portion (rectus ventralis, r.v) arising from the last sternal rib and inserted into the ischium : an external portion (rectus lateralis, $r . l$ ) beginning as a thin sheet of fibrez between the skin and the pectoral muscle (\$ 127) 


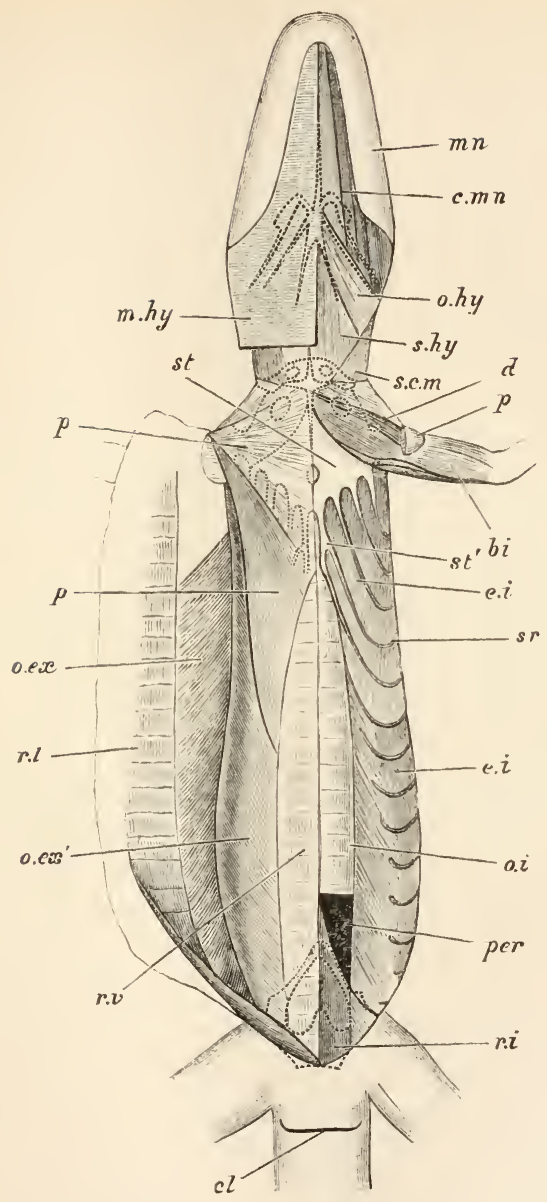

FIG. 39.-Lacerta viridis. The chief muscles of the ventral aspect of the body (nat. size). On the right side the skin is reflected outwards along with the rectus lateralis $(r . l)$ and superficial layer of the external oblique $(0 . e x)$. 
The position of the hyoidean apparatus, clavicles, interclavicle, coracoid, and right half of the sternum, and of the pubes and ischia, are indicated by dotted outlines. On the left side (right in the figure), the mylohyoid $(x . h y)$, pectoral $(p)$, and external oblique $(o . e x)$ muscles are removed, as well as the posterior part of the rectus ventralis $(r . v)$ : by the removal of the pectoral the left half of the sterum $(s t)$ is exposed.

$b i$, biceps : $c l$, cloacal aperture : $c . m n$, cerato-mandibularis : $d$, deltoid: e.i, external intercostal: $m . h y$, mylo-hyoid: $m n$, mandible : $0 . e x$, obliquus externus, superficial portion: $0 . e x^{\prime}$, deep portion of the same : $0 . h y$, omo-hyoid : $0 . i$, obliquus internus: $p$, pectoralis: per, peritoneum : $r_{.} i$, rectus internus : $r . l$, rectus lateralis : $r . v$, rectus ventralis : s.c.m, sterno-cleido-mastoideus: s.hy, sterno-hyoideus : sr, serratus : st, sternum : $s t^{\prime}$, its posterior prolongations.

and joining the preceding portion at its insertion : and an internal and posterior portion (rectus internus, r.i) of triangular form, lying between the ventral portion and the peritoneum, attached behind to the puboischiatic ligament and gradually thinning out in front.

I26. The obliquus externus $\left(0 . e x, o . e x^{\prime}\right)$, a thin sheet of muscle lying external to the rectus, and with its fibres taking a direction downwards and backwards (the animal being supposed to be in the normal attitude).

It consists of two layers, a superficial one (o.ex) arising by slips from the vertebral ribs, and with its fibres ending against the outer border of the rectus lateralis, with which it becomes indistinguishable posteriorly ; and a deep portion (o.ex') lying within (dorsal to) the pectoral, and similarly related to the rectus ventralis.

I 27. The pectoralis $(p)$, a large paired muscle arising from the sternum and sternal ribs, which it largely covers: the fibres of its anterior part pass outwards and backwards, those of its posterior portion outwards and forwards, those of its middle region directly outwards, and, converging, are inserted into the ventral aspect of the humerus, thus forming the chief depressor of the arm.

I 28. The mylo-hyoid ( $m . h y)$, a strong sheet of transverse muscular fibres passing from ramus to ramus of the lower jaw. 
XV. Cut through the origins of both pectoral muscles and reflect them outwards: dissect away the external oblique and note

I29. The ribs and sternum, and their relation to the body walls (see $\S \S 22-25$ and Fig. 39).

I30. The external intercostal muscles (e.i), filling up the interspaces between the ribs: their fibres run from any given rib backwards and slightly downwards to its successor.

I3I. The obliquus internus $(0 . i)$, a thin sheet of muscle lying between the intercostals externally and the rectus ventralis internally, and exposed by the removal of the external oblique : its fibres pass downwards and forwards.

XVI. Make a longitudinal incision through the rectus ventralis, a litile to one side of the median line, taking care not to injure the epigastric vein (§ 138$)$ : continue the incision backwards to the pubes, and forwards by cutting through the sternum and interclavicle: carefully separate the epigastric vein from the surrounding tissues and reflect the body walls. Note

I32. The cœlome or body cavity, containing the viscera, extending in front to about the level of the interclavicle, and behind continued beyond the anterior boundary of the pubes as the pelvic cavity.

I33. The peritoneum (parietal layer), a thin membrane lining the colome: in the posterior half of the cavity it is deeply pigmented, in the anterior half colourless, the boundary line between the black and colourless portions being perfectly sharp.

I34. The liver (Fig. 40, Ir), a large, brownish organ, occupying about the middle third of the body cavity, and imperfectly divided into right and left lobes. 


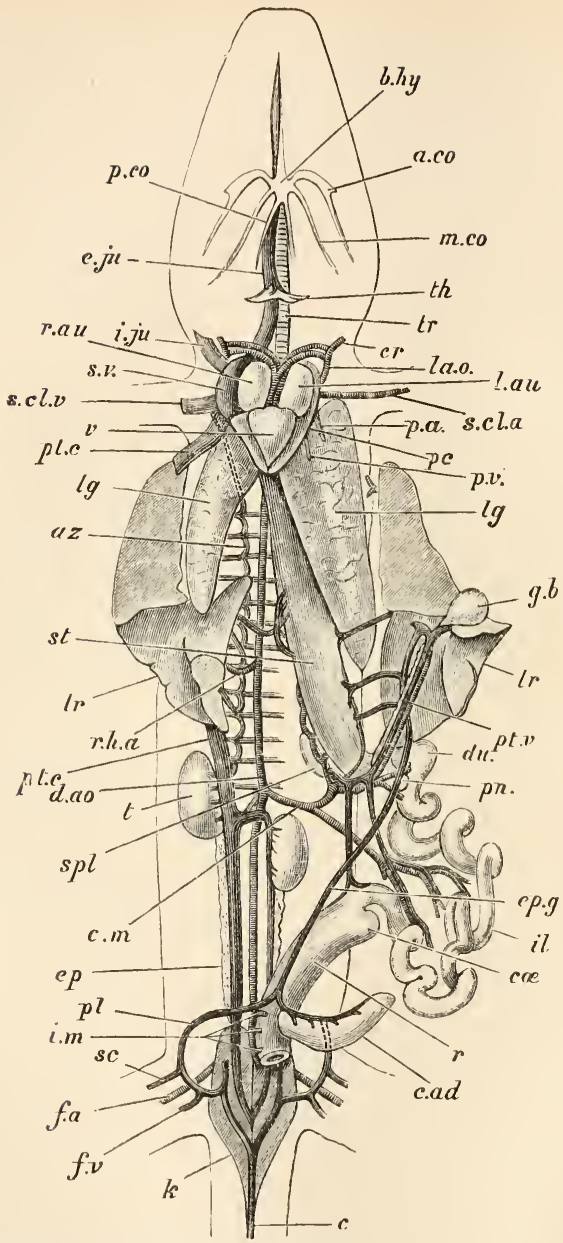

FIG. 40.-Lacerta viridis. $\Lambda$ dissection from the ventral aspect, showing the alimentary, circulatory, respiratory, and urinogenital organs (nat. size). The liver ( $\left(r^{\circ}\right)$ is divided longitudina'ly (see $§$ XVIII.), and 
its two halves displaced outwards: the alimentary canal is drawn out to the animal's left ; the cloaca, with the urinary bladder and posterior ends of the vasa deferentia, is removed, as also is the right corpus adiposum.

a.co, anterior cornu of hyoid : $a z$, azygos, or cardinal vein : $b . h y$, bcdy of hyoid : $c$, caudal vein : $c . a d$, corpus adiposum : $c . m$, cœliacomesenteric artery, the branches of which have no reference letters, to avoid confusing the figure, (: ee § I59) : $c c$, cæcum : $c r$, carotid artery : $d . a o$, dorsal aorta: $d u$, duodenum: e.ju, external jugular vein : $e p$, epididymis : efg, epigastric vein : f.a, femoral artery $: f . v$, femoral vein : $g . b$, gall-bladder $: i . j u$, internal jugular vein : $i l$, ileum $: i . m$, inferior mesenteric arteries $: k$, kidney $:$ l.ao, left aortic arch : l.au, left auricle : $l g$, lur.gs : $l r$, liver : $m . c o$, middle cornu of hyoid : $p . a$, pulmonary artery : $p c$, pericardium : $p . c o$, posterior cornu of hyoid : $p n$, pancreas : $p l$, pelvic vein : $p t . c$, postcaval rein : $p t . v$, portal vein : $p . v$, pulmonary vein : $r$, rectum : r.au, right auricle : $r . h . a$, right hepatic artery : sr, sciatic vein : scl.a, sul clavi:n artery : scl.v, subclavian vein : $s p l$, spleen : $s t$, stomach : s. $v$, sinus venosus : th, thyroid gland : $t r$, trachea : $t s$, testis : $v$, ventricle.

135. The gall-bladder $(g, b)$, an ovoiclal sac, situated at the lower margin of the right lobe of the liver, and having, in the fresh state, a green colour, due to the contained bile.

I36. The intestine $(d u$, il), forming several coils in the posterior third of the cœlome.

137. The corpora adiposa (c.ad), paired masses of fat, of somewhat semi-lunar shape, lying immediately in front of the anterior boundary of the pelvis, between the peritoneal and muscular layers of the body-wall.

I38. The epigastric or anterior abdominal vein $(e p s)$, a median ventral vessel, running along the inner face of the body wall, between the peritoneum and the rectus abdominis: posteriorly it is formed by the union of two pelvic veins $(p l)$, which are attached to the inner borders of the corpora adiposa and receive veins from them: anteriorly the epigastric vein enters the left lobe of the liver on its posterior face : two other abdominal veins bring blood from the ventral body wall, and enter the liver on its ventral face at about the junction of its right and left lobes. 
I39. The heart $(v$, r.au, l.au), situated a short distance in front of the liver and enclosed in a delicate membrane, the pericardium. (For the characters of the heart see $\$ \$ 197$ -200.)

I 40. The postcaval vein (pt.c), a large vessel, usually gorged with blood, passing from the anterior border of the liver forwards to the right side of the heart. ${ }^{1}$

I $4 \mathrm{I}$. The lungs $(l g)$, two transparent, thin-walled sacs lying one on each side of the postcaval vein between the heart and the liver (see $\S 2$ I 3 ).

I42. More or less of the reproductive organs can usually be seen even without moving the intestines: by turning the latter aside the testes of the male $(\$$ I 66) and the ovaries ( $(169)$ and oviducts ( $(70)$ of the female, are displayed.

143. A large vein is also seen, by turning aside the intestines, lying between the testes or ovaries, and passing forwards to the postero-dorsal region of the liver; this is the hinder part of the postcaval (pt.c).

XVII. Dissect away the mylo-hyoid (m.hy) and underlying muscles (c.mn, o.hy, s.hy, $\mathcal{S}_{0 .}$ ) until the following structures are laid bare:

I 44. The trachea $(t r)$, an annulated tube lying in the middle ventral line of the throat.

1 The injection of the lizard's vascular system is conveniently done as follows : Kender the animal insensible with chloroform : lay bare the heart as directed ( $\$ \mathrm{XVI}$ ), taking care not to injure the epigastric vein : slit open the pericardium, and cut off the apex of the ventricle ( $\$$ 197). When the bleeding hasstopped, $\mathrm{pu} \mathrm{h}$ a cannula through the wound into the cavity of the ventricle and thence into the right aorta ( $\$ 199)$, and tie it in place by a ligature round the base of the ventricle. A warm solution of gelatine coloured with carnine, vermilion, or French blue (ultramarine) is the best injecting medium; it is firm enough to pass through capillaries, so that the whole vasiular system, with the exception of the pulmonary vessels, can be injected at one operation. 
I 45. The thyroid gland (th), a whitish, transversely elongated body on the ventral wall of the trachea, a short distance in front of the heart.

I46. The right precaval vein (Fig. 43, p. I72, pr.c), situated in the lateral region of the neck, and passing backwards towards the right side of the heart (see $\S 200$ ): it is formed by the union of three veins; the internal jugular (Figs. 40 and $43 i . j u$ ), coming from the lateral region of the head and bringing blood from the brain, \&c. ; the external jugular $(e . j u)$, situated in the middle ventral line of the throat, and at its hinder end turning to the right to join the internal jugular; and the subclavian $\left(s c l . \tau^{\prime}\right)$, bringing blood from the right fore-limb.

I47. The left precaval, formed by the union of the left internal jugular and left subclavian veins: the left external jugular is absent.

I 48. The position and relation of the hyoidean apparatus (\$ $\$ 9-83)$.

XVIII. Make a longitudinal vertical incision through the liver, beginning at its anterior border, a little to the left (the animal's left, your right) of the postcaval, and passing backwards to the right of the epigastric vein and gall-bladder: in this way the whole postcaval is left uninjured on the right side, and the gall-bladder, with its duct ( $\left(\mathbf{1}_{65}\right)$, and portal

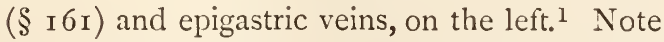

149. The stomach (Fig. 40, st), hitherto concealed beneath the liver: it is a tubular organ, of slightly greater diameter than the intestine, and with thick, muscular walls.

1 This method of dissection is adopted because of the connection of the liver to the dorsal body-wall by blood-vessels (\$§ 163, 164), which are ruptured if the liver is turned forwards in the ordinary way : in fresh, uninjected specimens there will of course be a good deal of bleeding, but this objection does not apply either to injected or to spirit specimens. 
I50. The divisions of the intestines: there is no distinction between the duodenum $(d u)$ or section immediately succeeding the stomach and the ileum (il), or remainder of the small intestine : the latter, after one or two turns, passes into the large intestine, or rectum $(r)$, distinguished by its greater diameter and by having its anterior end produced on the left side into a small blind pouch or cæcum.

I51. The pancreas $(p n)$, an elongated, whitish gland, situated in the loop between the stomach and duodenum, and continued forwards to the posterior face of the left lobe of the liver.

I52. The gastro-hepatic omentum, a thin sheet of peritoneum extending from the dorsal surface of the liver to the ventral surface of the stomach, and continued backwards by the duodeno-hepatic omentum, which similarly connects the liver with the first loop of the intestine.

I 53. The mesogaster, a fold of peritoneum connecting the stomach with the dorsal body-wall.

I 54. The mesentery, a similar fold suspending the small intestine.

I55. The mesorectum, a backward continuation of the mesentery suspending the rectum.

156. A similar fold attached to the free or dorsal edge of the right lobe of the liver.

I57. The spleen ( $s p l)$, a small, red body, lying in the mesogaster, near the posterior end of the stomach.

I58. The dorsal aorta (d.ao), an unpaired longitudinal artery lying in the middle dorsal line.

159. The cœliaco-mesenteric artery $(\mathrm{c} . \mathrm{m})$, given off from the dorsal aorta at about the level of the anterior end of the right testis, or ovary, and soon dividing into two trunks, an anterior, the cœliac artery, supplying the 
stomach, spleen, pancreas, duodenum, and left iobe of the liver; and a posterior, the superior mesenteric artery, which supplies the ileum.

160. The inferior mesenteric arteries $(i . m)$, three small vessels arising from the aorta at about the level of the pubes and supplying the rectum.

I61. The portal vein $\left(p t \cdot v^{\prime}\right)$, a large vessel lying in the duodeno-hepatic omentum, parallel to the pancreas: it receives gastric veins from the stomach, and small pancreatic veins from the pancreas, and at its distal end is formed by the union of splenic and mesenteric veins from the spleen and intestine respectively.

162. The separate gastric portal veins, two or three small vessels taking blood directly from the stomach to the liver.

163. Two small veins taking blood from the dorsal body-wall to the right lobe of the liver : by dissecting off the peritoneum they are seen to spring from the azygos (cardinal) vein (az), a longitudinal trunk lying on the right side of, and close to the vertebral column, which receives blood from the dorsal region of the trunk, intercostal spaces, \&c., and joins the precaval close to the entrance of the subclavian.

164. The right hepatic artery $(r . h . a)$, a small vessel springing from the dorsal aorta, and passing parallel to the veins mentioned in $\S 16_{3}$ to the right lobe of the liver.

165. The common bile-duct, running parallel to the portal vein and opening posteriorly into the duodenum : at its anterior end it is formed by the union of the cystic duct from the gall-bladder and the hepatic duct from the liver itself.

\section{In the male:-}

I66. The testes (Figs. 40 and $4 \mathrm{I}, t$ ), two white ovoidal bodies, that on the right side situated just posterior to the right lobe of the liver, that on the left somewhat further back.

r67. The epididymis (ep), a whitish, elongated body attached to the inner face of each testis, and extending backwards into the pelvic cavity (see $\$$ I90). 
I68. The mesorchium (Fig. 4I, ms.o), a fold of peritoneum suspending each testis to the dorsal body-wall : a similar fold $(b . \lg )$ supports the epididymis, and is attached in front along the line of junction of the colourless and pigmented regions of peritoneum (\$ I 33).

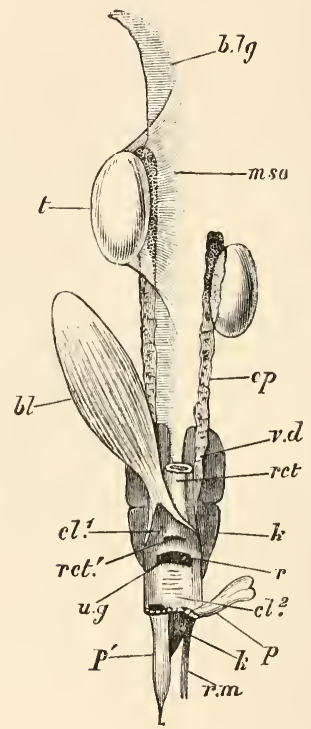

FIG. 41.-Lacerta viridis. The male urinogenital organs (nat. size). The ventral wall of the cloaca is removed, the bladder is turned to the animal's right, and the peritoneal covering of the left testis and epididymis is dissected away.

$b l$, urinary bladder $: b l g$, fold of peritoneum supporting epididymis : $c l^{t}$, anterior, and $c l^{2}$, posterior, division of cloaca : $s p$, epididymis : $k$, kidney: $m s . o$, mesorchium: $p$, copulatory organs, of which the right is shown retracted $\left(p^{\prime}\right)$ and the left everted $(p): r . m$, retractor muscle of latter : $r$, ridge separating anterior and posterior divisions of cloaca $: r c t$, rectum : $r t^{\prime}$, its opening into the cloaca : $t$, testis: $u \cdot g$, urinogenital papilla and aperture : $v . d$, vas deferens. 
In the female:-

I69. The ovaries (Fig. 42, ov) situated further back than the testes, the left being partly within the pelvic cavity: their surface is raised into hemispherical elevations, due to the ova.

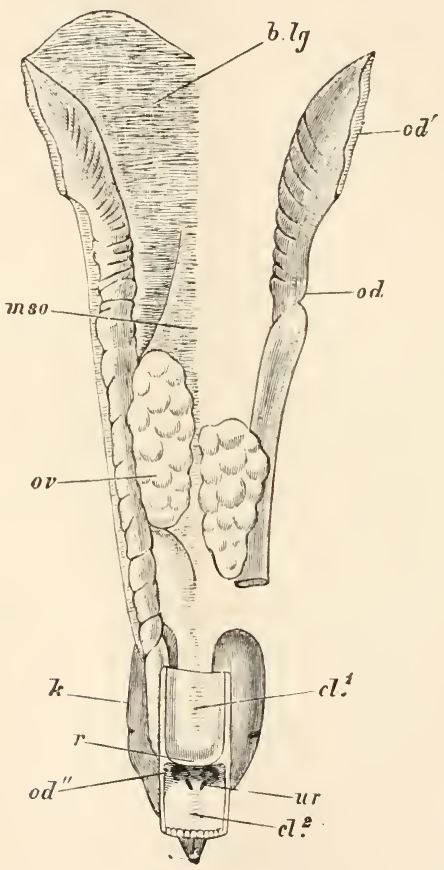

FIG. 42.-Lacerta viridis. The female urinogenital organs (nat. size). The ventral wall of the cloaca, the urinary bladder, the posterior end of the left oviduct, and the peritoneal investment of the left ovary and oviduct are removed.

b.lg, broad ligament: $c l^{\mathrm{x}}$, an'erior, and $\mathrm{cl}^{2}$, posterior, division of cloaca : $k$, kidney : $m$ s.o, mesoarium $: o d$, left oviduct $: o d$, its peritoneal aperture : $o d^{\prime \prime}$, aperture of right oviduct into the cloaca : ov, ovary: ur, aperture of ureter. 
I 70. The oviducts (od), delicate, plaited tubes, lying dorsal and external to the corresponding ovaries, and extending backwards into the pelvic cavity : for their further characters see $\S$ I 94 .

I 7 I. The mesoarium (ms.o), a fold of peritoneum suspending each ovary to the dorsal body-wall.

I72. The broad ligament (b.lg), a fold similarly suspending the oviduct: its anterior line of attachment corresponds with the junction between the colourless and pigmented regions of the peritoneum ( $\$$ I33).

\section{In both sexes:-}

I73. The postcaval vein can now be seen to be formed by the union of two veins lying within and parallel to the epididymes or oviducts, and of which the right is considerably larger than the left (Fig. 40).

i 74. The whole extent of the lungs can now be seen : they extend backwards to about the level of the gall-bladder, the right fitting into a notch on the dorsal face of the right lobe of the liver.

175. The pleura, a fold of peritoneum connecting each lung with the dorsal body-wall.

XIX. Dissect off the muscles covering the ventral surface of the pelvis, and cut away the median portion of the pubes and ischia : also dissect away the muscles attached to the inner surfaces of these bones, portions of which will still be left obscuring the contents of the pelvic cavity. Note

176. The cloaca (Fig. 4I, $c l^{1}, c l^{2}$ ) or posterior division of the alimentary canal, passing insensibly into the rectum in front, and opening behind by the vent.

177. The urinary bladder $(b l)$, a thin-walled sac attached by its posterior extremity or neck to the ventral 
wall of the cloaca, and having a bilobed free anterior extremity or fundus.

I 78. The kidneys (Figs. 40-42, k), two dark, reddishbrown bodies, situated between the dorsal wall of the pelvic cavity and the rectum : their anterior ends are about on a level with the corpora adiposa, their posterior ends reach back a little beyond the level of the vent : they are irregular in shape, adapting themselves to the form of the cavity in which they lie, and their tapering posterior ends are closely applied to one another: they are covered by peritoneum on the ventral face only: each consists of two lobes, an anterior and a posterior.

I79. The pelvic veins (Fig. $40, p l$ ) have already been seen $\left(\$ \mathrm{I}_{3} 8\right)$ attached to the edges of the corpora adiposa : traced backwards, each is szen to pass across the ventral face of the corresponding kidney, and to be united with its fellow to form a single trunk: this latter, which by its bifurcation forms the pelvic veins, is the caudal vein $(c)$; it lies along the ventral aspect of the caudal vertebræ and brings back blood from the various tissues of the tail.

I80. The principal renal portal vein, arising from the pelvic vein at the point where it turns outwards across the ventral face of the kidney: it enters the anterior lobe of the kidney, supplying it with venous blood: the posterior lobe is supplied by smaller renal portal veins springing from the hinder part of the pelvic veins.

I8r. The femoral $\left(f, v^{\prime}\right)$ and sciatic (sc) veins, two trunks, the former from the anterior, the latter from the posterior region of the leg, and opening into the corres. ponding pelvic vein.

I82. The two trunks which by their union form the postcaval vein ( $(173)$ : the right is considerably the larger and is directly continued into the postcaval, while 
the left crosses over to the right side to join its fellow at about the level of the origin of the cœliaco-mesenteric artery: each trunk receives renal veins from the corresponding kidney, and unites with its fellow just anterior to the bifurcation of the caudal vein.

I83. The femoral artery ( $f . a)$, passing outwards to the hind limbs between the femoral and sciatic veins : its origin from the dorsal aorta is best seen at a later stage.

$\mathrm{XX}$. Cut through the stomach a short distance behind the heart, and through the rectum a little anterior to its junction with the cloaca, and remove the alimentary canal between these two points: open the various parts of it by longitudinal incisions, and note

I 84. The rugæ of the stomach, strong longitudinal ridges into which its mucous membrane is produced, and the pyloric valve, an annular ridge of mucous membrane narrowing the aperture between the stomach and duodenum.

I85. The valvulæ conniventes, delicate longitudinal ridges on the mucous membrane of the small intestine.

I86. The ileo-cæcal valve, an annular ridge of mucous membrane narrowing the aperture between the small and large intestines, and projecting into the latter.

I 87. The aperture of the bile-duct in the duodenum.

XXI. Dissect away the peritoneum from the kidneys and reproductive organs, and open the cloaca by a ventral longitudinal incision a little to one side of the middle line, so as not to injure the bladder. Make out

I88. The division of the cloaca by an incomplete ridge of mucous membrane (Figs. $4 \mathrm{I}$ and $42, r$ ) into two sections, an anterior $\left(c l^{1}\right)$ receiving the rectum and a posterior $\left(c l^{2}\right)$ opening externally by the vent. 
I89. The aperture of the urinary bladder in the ventral wall of the anterior division of the cloaca.

In the male:-

I90. The vasa deferentia (Fig. 4r, $v \cdot d$ ), convoluted tubes, continuous with the epididymes in front, and passing backwards to the dorsal wall of the cloaca.

I9I. The urinogenital apertures $(u \cdot g)$, minute apertures each on the apex of a small papilla on the dorsal wall of the posterior division of the cloaca.

I92. By turning aside the cloaca the ureters may be seen as delicate tubes situated between the pelvic and renal veins, and uniting posteriorly with the vasa deferentia.

I93. The copulatory organs $(p)$, vascular eversible sacs, lying on the ventral side of the tail, immediately beneath the skin, and opening into the posterior part of the cloaca. When everted each is seen to be a somewhat cylindrical body, with a dilated and bifid apex.

In the female :-

I94. The paired apertures of the oviducts (Fig. 42, od") in the dorsal wall of the hinder division of the cloaca, just posterior to the dividing ridge: anteriorly the oviducts open into the colome by wide apertures $\left(o d^{\prime}\right)$.

I95. The apertures of the ureters (ur), small oblique slits in the dorsal wall of the cloaca, a little posterior to the oviducal apertures.

196. The ureters themselves are situated as in the male (\$ I 92$)$.

XXII. Lay open the pericardium and observe

I97. The ventricle (Figs. 40 and $43, v$ ), a fleshy conical structure, having its pointed apex directed backwards and connected to the pericardium by a fine ligament.

I98. The right (r.au) and left (l.au) auricles, thin- 
walled chambers, situated anterior to the ventricle, to the base of which they are united.

I99. The three great arteries, springing from the base of the ventricle, and passing forwards between the auricles, closely bound together with connective tissue: when the latter is dissected away they are seen to have a twist to the left. Of the three, the pulmonary artery (Figs. 43 and 44, p.a), lying to the animal's left, and the left aorta (l.ao) to the right, are situated ventrally at their origin, while the right aorta $(r . a o)$ is dorsal to the other

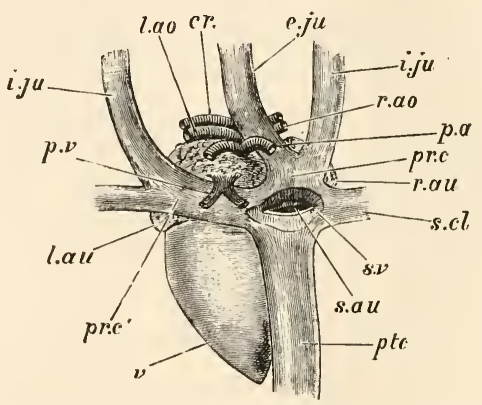

FIG. 43.-Lacerta viridis. The heart, from the dorsal side $\left(X_{3}\right)$. $c r$, carotid artery: e.ju, external, and $i . j u$, internal jugular vein : l.ao, left aorta : l.au, left auricle : p.a, pulmonary artery : pr.c, right, and $p r . c^{\prime}$, left, precaval vein : $p t . c$, postcaval vein : $p . v$, pulmonary vein : $r . a o$, right aorta $: r . a u$, right auricle : s.au, sinu-auricular aperture $: s . c l$, subclavian vein $: s . v$, sinus venosus, cut open to show the sinu-auricular aperture : $v$, ventricle.

two ; traced forwards the pulmonary artery is seen gradually to assume a dorsal position, the left aorta becoming ventral and to the left, the right aorta ventral and to the right.

200. The sinus venosus (s. $v)$, a chamber situated to the dorsal side of the right auricle, and seen by turning the heart aside; it receives the three great veins; the right 
precaval (pr.c) passing directly backwards, the postcaval ( $p$ t.c) directly forwards into it, while the left precaval ( $\left.p r . c^{\prime}\right)$, on reaching the neck, turns medianwards and passes across the dorsal wall of the left auricle.

20x. The division of the pulmonary artery as it pierces the front wall of the pericardium into a right and left trunk, each of which passes at first outwards and then backwards to the corresponding lung.

202. The pulmonary veins $(p . v)$, bringing the blood from the lungs, and uniting into a short common trunk which enters the left auricle on its dorsal wall.

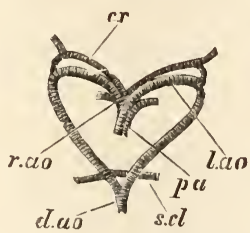

FIG. 44.-Lacerta viridis. The aortic arches, supposed to te removed close to their oris in from the heart (nat. size).

$c r$, carotid artery : d.al, dorsal aorta : l.ao, left aortic arch : p.a, pulmonary artery $: r . a o$, right aortic arch : $s . c l$, subclavian artery.

203. Leaving the pericardium, the two aortic arches (Fig. 44) separate from one another, the right arch turning to the right side, the left to the left: traced outwards they are seen to bend round the œsophagus and to unite with one another on the ventral face of the vertebral column to form the dorsal aorta (d.ao).

204. The carotid arteries ( $c r$ ), arising together from the right aorta just where it diverges from the left: each carotid passes outwards, parallel with the corresponding aortic arch, with which, at its distal end, it anastomoses 
(ductus Botalli): shortly before this union the carctid artery proper is given off and passes forwards to the head.

205. The subclavian arteries (s.cl), arising from the right aortic arch just before its junction with the left, and passing directly outwards to the fore-limbs.

XXIII. Remove the heart, and open its various cavities under water : first placing it on its ventral face and opening the sinus venosus from the dorsal side, and afterwards placing it on its dorsal face and opening the auricle and ventricle from the ventral side. Note

206. The thin, smooth walls of the sinus venosus.

207. The sinu-auricular aperture (Fig. 43, s.au), a transverse slit placing the sinus venosus in communication with the right auricle, and guarded by the two-lipped sinuauricular valve.

208. The septum auricularum, a vertical partition separating the auricles from one another.

209. The musculi pectinati, forming a network on the inner surface of the walls of the auricles.

2 ro. The auriculo-ventricular aperture, placing both auricles in communication with the ventricle, and divided into two by the free edge of the septum auricularum : guarding the aperture is the auriculo-ventricular valve, consisting of two semi-lunar flaps, a right and a left, placed back to back in such a way that each shuts across one half of the auriculo-ventricular aperture.

2 II. The thick, spongy walls and small cavity of the ventricle.

2 I2. The origins of the great arteries: the pulmonary artery arises to the left of the two aortæ, and its aperture is separated from theirs by a muscular partition: the apertures of the aortic arches are in the same vertical plane, that of 
the left being ventral to that of the right: all three arterial apertures lie to the left of the auriculo-ventricular aperture.

XXIV. By the removal of the heart, the lungs are thoroughly exposed; note in them the following points :-

2 I3. Each lung is a fusiform sac, very thin in its posterior half, much thicker anteriorly: on cutting it open, the wall is seen to be raised into a network of delicate ridges, producing a honey-combed appearance: the network is closer and the ridges more marked at the anterior than at the posterior end of the lung.

2I4. The bifurcation of the trachea into two very short tubes, the bronchi, which communicates each with the corresponding lung by a small aperture a short distance from its apiculate anterior end.

2 I 5 . The complete cartilaginous rings of the trachea and bronchi.

$X X V$. Slit up what remains of the gullet along one side, and continue the incision forwards, through the floor of the mouth, close alongside the inner edge of the mandible; turn aside the floor of the mouth so as to expose the oral cavity: if the latter is not sufficiently displayed, part of the mandible may be cut away with bone forceps.

Observe

2r6. The tongue, a narrow, elongated, fleshy organ on the floor of the mouth, bifid in front.

217. The glottis, or aperture of the trachea into the mouth, a small longitudinal slit, placed at the summit of a rounded prominence in which are contained the cartilages of the larynx.

2 r8. The small, conical teeth, arranged in a single series round the upper and lower jaws (premaxillary, maxillary, 
and mandibular teeth): there is also a small patch of teeth on each side of the roof of the mouth (palatine teeth).

2in. The posterior nares, considerable apertures situated a little behind the end of the snout and separated from one another by a vertical partition supported by the vomers ( $\left.\$ 6_{3}\right)$.

Slightly in front of the posterior nares are two minute slit-like apertures, each leading into a small pigmented sac (Jacobson's organ) lying under the nasal sac. The relations of these parts are best seen in a transverse section of the snout.

220. The Eustachian pits; large paired diverticula of the dorsal wall of the plarynx, continuous with the tympanic cavities, which are bounded externally by the tympanic membranes.

221. The position of the columella auris in the tympanic cavity, and its relations to the fenestra ovalis and tympanic membrane, may now be made out (see $\S 65, p$. I42). Also remove an eye aı d note a series of thin scale-like bones (sclerotic plates) surrounding the border of the sclerotic.

XXVI. Break away the roof of the skull with bone forceps so as to expose the brain: this may be done without first dissecting off the skin which adheres very closely to the subjacent bones. Remove the brain, taking especial care not to injure the olfactory lobes (\$227, Fig. 45, olf), and, after preservation in spirit, place it under water in a small dissecting dish, and make out its parts as follows :-

222. The medulla oblongata (Fig. 45, m.o), or postericr division of the brain, continued insensibly behind into the spinal cord: it is about double the diameter of the cord, and exhibits a strong ventral flexure. 

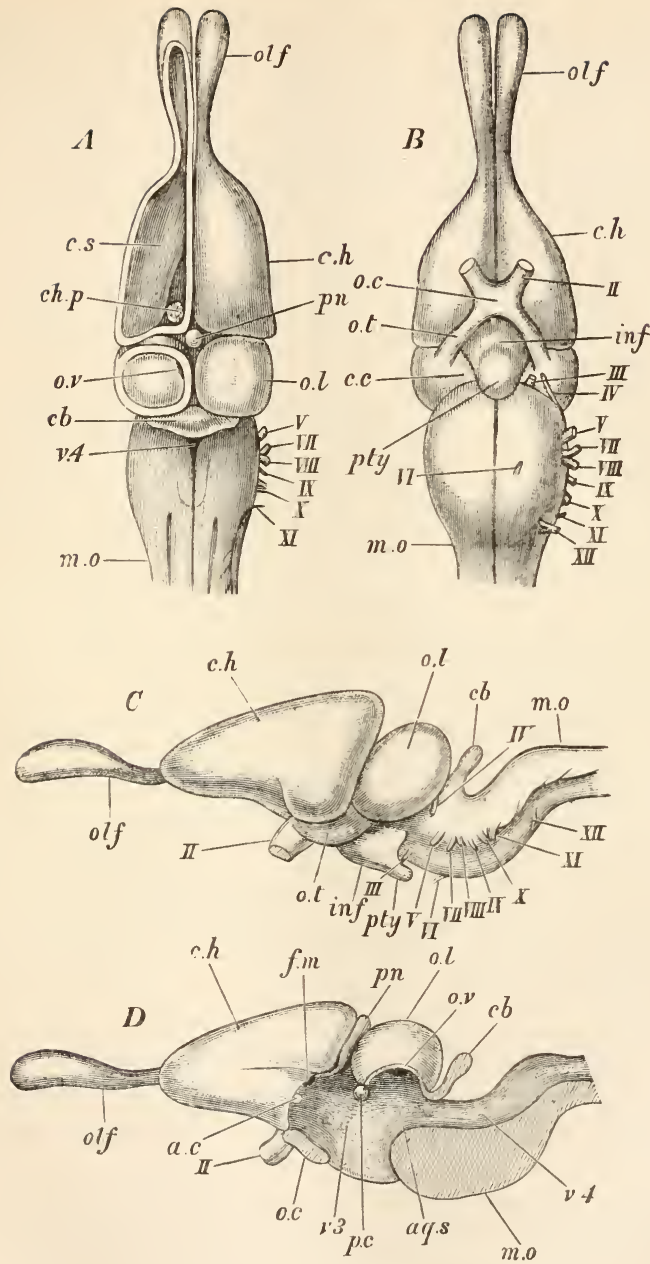

FIG. 45.-Lacerta viridis. The brain; A, from above, with the left cerebral hemisphere $(c . h)$ and optic lobe $(o . l)$ opened; $\mathrm{B}$, from beneath; $c$, from the left side; $D$, in longitudinal vertical section. 
a.c, anterior commissure : aq.s, aqueduct of Sylvius : $c b$, cerebellum : c.c, crura cerebri : c.h, cerebral hemispheres : ch.p, choroid plexus : c.s, corpus striatum : $f . m$, foramen of Monro: inf, infundibulum : $m . o$, medulla oblongata : o.c, optic chiasma : o.l, optic lobes : olf, olfactory lobes : o.t, optic tracts: $0 . v$, aperture between aqueduct of Sylvius and optic ventricle : $p . c$, posterior commissure : $p n$, pineal body : $p t y$, pituitary body : $v .3$, third ventricle : v.4, fourth ventricle : $I-X I I$, cerebral nerves.

223. The cerebellum $(c b)$, a small semicircular flap covering the anterior part of the medulla oblongata on the dorsal side.

224. The fourth ventricle ( $(1.4)$, a cavity on the dorsal surface of the medulla, roofed over in front by the cerebellum, but for the rest of its extent covered only by the pia mater, a delicate membrane investing the brain and spinal cord.

225. The optic lobes, or corpora bigemina $(0 . l)$, two large rounded elevations on the dorsal surface of the brain, immediately in front of the cerebellum : they constitute the dorsal portion of the mid-brain, the ventral region of which answers to the crura cerebri (c.c).

226. The cerebral hemispheres $(c . h)$, large, pyramidal paired masses, immediately in front of the optic lobes, against the anterior faces of which they abut closely: they are in close contact with one another by their flat inner faces, and are pointed in front.

227. The olfactory lobes (olf) paired, club-shaped bodies continued forwards from the anterior ends of the cerebral hemispheres: from their dilated anterior ends are given off the olfactory (first cerebral) nerves to the nasal sacs.

228. The pineal body $(p n)$, a small, rounded mass in the middle line of the dorsal surface of the brain, at the junction of the cerebral hemispheres and optic lobes.

229. The pituitary body ( $p t y)$, a rounded mass situated 
in the middle line of the ventral surface of the brain, immediately under the pineal body, and attached to an elevation, the infundibulum.

230. The thalamencephalon, seen by slightly separating the posterior ends of the cerebral hemispheres as a rounded median mass, interposed between the hemispheres and the optic lobes: the infundibulum is a ventral, backwardly-directed prolongation of the thalamencephalon.

23I. The optic chiasma (o.c), an x-shaped body situated immediately anterior to the infundibulum, and consisting of the optic tracts $(0 . t)$, which pass backwards and slightly upwards on either side of the infundibulum to the anterior ventral region of the optic lobes, and of the optic (second) nerves (II), which, becoming free from the brain, pass forwards and outwards to the eyes.

232. The third nerve (III), arising from the ventral surface of the mid-brain.

233. The fourth nerve (IV), arising from the dorsal surface of the medulla oblongata, just in front of the cerebellum.

234. The fifth nerve, or trigeminal (V), arising from the ventrolateral region of the medulla.

235. The sixth nerve (VI), a small nerve arising from the ventral region of the medulla, not far from the middle line.

236. The seventh nerve (VII), arising in common with the eighth from the lateral surface of the medulla, just posterior to the fifth.

237. The eighth, or auditory nerve (VIII), arising in common with the seventh.

238. The ninth nerve, or glossopharyngeal (IX), arising immediately behind the eighth, and at a somewhat lower level.

239. The tenth nerve, or vagus $(\mathrm{X})$, arising just posterior to the ninth, by three or four separate roots.

240. The eleventh nerve, or spinal accessory (XI), arising from the spinal cord, and passing forwards, receiving fibres as it goes, to the medulla oblongata, which it finally leaves immediately behind the vagus.

24I. The twelfth nerve, or hypoglossal (XII), arising a little behind the eleventh from the ventral surface of the medulla. 
XXVII. Carefully cut into the cerebral hemisphere and optic lobe of one (say the left) side, ${ }^{1}$ and remove the roof of the cavities exposed: observe :

242. The optic ventricle (Fig. 45, A), a small cavity in the thick-walled optic lobe: in its inner wall is a small aperture $(0 . v)$, which leads from the aqueduct of Sylvius $(\S 246)$.

243. The lateral ventricle, a considerable cavity in the cerebral hemisphere, extending in front into the olfactory lobe: its dorsal and inner walls are thin ; its ventrolateral wall is greatly thickened so as to form a well-marked eminence, the corpus striatum (c.s).

244. The choroid plexus (ch.p), a plaited fold of connective tissue, abundantly supplied with blood-vessels, lying in the postero-internal angle of the lateral ventricle.

245. Traced downwards, the choroid plexus is seen to pass through the foramen of Monro $(D, f . m)$, a small aperture in the posterior part of the inner wall of the lateral ventricle, and communicating with the third ventricle $(\S 248)$ : to see it the corpus striatum must be drawn aside.

XXVIII. Remove the left half of the cerebellum and enough of the left optic lobe and cerebral hemisphere to bring into view

246. The aqueduct of Sylvius (aq.s), a passage bounded below by the crura cerebri and above by the optic lobes, in free communication with the fourth ventricle posteriorly, and communicating by the small lateral apertures already seen $(\S 242)$ with the optic ventricles, with which it forms the ventricle of the mid-brain, or mesocœle.

247. The optic thalami, paired masses of which the thalamencephalon is composed.

3 If another specimen is to be dissected, this may be done on both sides, and a longitudinal section ( $\$$ XXIX) made of the second brain. 
248. The third ventricle $(\% \cdot 3)$, a narrow vertical slit between the optic thalami, continuing forwards the aqueduct of Sylvius: it communicates in front by the foramen of Monro $(f . m)$, already seen $(\$ 245)$, with the lateral ventricle. 249. The anterior commissure (a.c), a small, transverse band of white nerve fibres, passing transversely between the two optic thalami, below and somewhat in front of the foramen of Monro.

250. The posterior commissure (p.c), a similar transverse band connecting the optic thalami at their junction with the mid-brain.

XXIX. Cut away the remainder of the left side of the brain so as to get a longitudinal vertical section of - the whole organ (Fig. 45, D). Note:

$25 \mathrm{I}$. The continuity between the fourth ventricle, aqueduct of Sylvius, and third ventricle, and the precise position of the apertures leading into the lateral and optic ventricles. 252. The downward continuation of the third ventricle into the infundibulum.

253. The general relations of the parts seen in the previous dissection. 


\section{THE P I G E O N.}

\section{(Columba livia.)}

\section{A. -THE SKELETON.}

I. Two skeletons should be examined, one of an adult bird, the other of a nestling two or three weeks old, the latter being especially useful for the skull, sacrum, pelvis, \&c., which, owing to the early ankylosis of the bones, cannot be satisfactorily made out in the adult. The adult skeleton is best prepared by maceration, that of the nestling may be either macerated for a short time, or subjected to the action of boiling water for a few seconds : it must be kept in alcohol or glycerine and water, or prepared by the glycerine jelly process. In the adult skeleton the cervical vertebræ $(\S \mathrm{I})$ should be separated from one another and strung together in order: the limb bones should be separated on one side, on the other left attached by their ligaments: the ribs $(\$ 25)$ are best separated at the junction of their vertebral and sternal portions, the former being left attached to the vertebræ, the latter to the sternum; a longitudinal vertical section should be made of the skull with a fine fret-saw. 
II. Notice, first of all, the general composition of the skeleton: it consists of:

r. The vertebral column, distinguishable into (a) thirteen or fourteen cervical vertebræ, forming the skeleton of the neck, and the last two of them bearing welldeveloped ribs, which do not unite with the sternum; (b) the four anterior thoracic vertebræ, all united with one another and bearing ribs which articulate with the sternum; (c) the compound sacrum, consisting of several vertebræ ankylosed together, and giving attachment to the pelvis; of these ankylosed vertebræ the first is thoracic, bearing ribs which unite with the sternum, the next two or three are lumbar, the next three or four sacral, and the remaining six caudal; $(d)$ the free caudal vertebræ, about six in number; and $(e)$ the pygostyle or ploughshare bone, a laterally compressed plate, forming the posterior termination of the vertebral column.

2. The skull, consisting of (a) the brain-case and upper jaw ; $(b)$ the lower jaw or mandible; and $(c)$ the hyoid or tongue-bone.

3. The sternum, a broad, keeled plate, giving attachment to the five pairs of thoracic ribs.

4. The skeleton of the wings or fore-limbs, articulating with the shoulder-girdle, which consists of two bones on each side, the ventral one articulating with the sternum, and connected with its fellow by the U-shaped furcula or merrythought.

5. The skeleton of the hind-limbs or legs, articulating with the pelvis or hip-girdle, the two halves of which are, in the adult, firmly united with the sacrum.

III. Examine the last cervical vertebra, and make out the following parts :

6. The centrum or body of the vertebra, elongated 
from before backwards, flattened from above downwards at its anterior end, almost square in section posteriorly: its anterior and posterior faces by which it articulates with the preceding and succeeding vertebræ respectively are cylindroidal, the anterior face being concave from side to side and convex from above downwards, the posterior face convex from side to side and concave from above downwards.

7. The neurapophyses, upwardly directed processes, rising one on each side from the dorsal surface of the centrum, and meeting with one another in the middle line above, forming the neural arch, thus helping to close in the neural canal in which the spinal cord is contained. Each neurapophysis is slightly excavated in front, considerably excavated behind, so that in the articulated vertebral column a series of intervertebral foramina are left between successive neurapophyses and serve for the exit of the spinal nerves.

8. The neural spine, a median vertical plate arising from the dorsal surface of the neural arch.

9. The anterior zygapophysis, a stout forwardly directed process, arising from the front edge of each neurapophysis and bearing a flat articular facet which looks upwards and inwards, and is overlapped by the posterior zygapophysis of the vertebra next in front.

Iо. The posterior zygapophysis, a sinilar, backwardly directec process arising from the hinder edge of each neurapophysis: it bears an articular facet which looks downwards and outwards, and overlaps the anterior zygapophysis of the vertebra next behind.

I I. The transverse process, a stout, outwardly directed rod springing from the outer face of each neurapophysis: it bears on its under surface a small tubercular facet for the tubercle of the rib (\$ I 4 ). 
I2. The capitular facet, a small articular surface on the lateral surface of the centrum near its anterior end, and giving articulation to the head of the rib ( $\$$ I 4 ).

I3. The hypapophysis, a vertical downwardly directed process, proceeding from the middle vertical line of the centrum.

I4. The ribs, small curved bones articulating, one on each side, with the vertebra, and tapering from their proximal to their distal ends. The proximal end is divided into two processes, one ventral, the head or capitulum, which articulates with the capitular facet on the centrum, the other dorsal, the tubercle, which articulates with the tubercular facet of the transverse process.

I5. The uncinate process, a small flat plate of bone given off from the posterior border of each rib, and directed backwards and upwards.

IV. In the remaining cervical vertebræ note:

I6. The gradual diminution from behind forwards of the neural spines, transverse processes, and hypapophyses, and the gradual elongation of the centra as far as about the third or fourth vertebra.

I7. In the penultimate vertebra the rib is much smaller than in the last, has no uncinate process, and its proximal end is indistinctly divided into head or tubercle.

I 8. The absence of free ribs in the remaining vertebræ: the apparent transverse process is, however, perforated by an aperture, the vertebrarterial canal, for the passage of the vertebral artery (Fig. 54, p. 232,vr), and the part of the transverse process external to this aperture represents a cervical rib, the head and tubercle of which have ankylosed with the centrum and transverse process respectively.

I9. The characters of the second vertebra or axis: it is considerably reduced in all dimensions: the anterior zyga- 
pophyses are very small and placed low down on the arch : the anterior face of the centrum is somewhat excavated and gives off dorsally a conical peg, the odontoid process, which bears an oblique articular facet on the ventral surface of its free end, for articulation with the atlas (\$20).

In the young bird the odontoid is a separate bone.

20. The characters of the first vertebra or atlas: it is very small and is reduced to a mere ring, the centrum being no thicker than the arch: the centrum is crescentic, the concavity of the crescent being upwards, and bearing an articular facet for the odontoid process of the axis, the two together forming a concave articular cavity for the occipital condyle $(\$ 35)$ : there are no anterior zygapophyses and the posterior zygapophyses are small.

21. The mode of articulation of contiguous centra: they are united by rings of cartilage, their adjacent faces being separated by a closed space (synovial capsule) : each intervertebral ring is produced inwards into a thin plate of cartilage, the meniscus, which is perforated in the centre, and forms a vertical partition dividing the synovial space into an anterior and a posterior division: a delicate ligamentous cord, the ligamentum suspensorium, passes from centrum to centrum through the aperture in the meniscus.

$\mathrm{V}$. In the thoracic vertebræ note:

22. The fusion of the first four into a single mass by the ankylosis of the centra, zygapophyses, and transverse processes.

23. The fusion of the fifth with the compound sacrum: its large transverse processes abut against the ilia, with which the proximal ends of its ribs are also united.

24. The large hypapophysis of the first thoracic vertebra, dividing below into two divergent laminæ.

25. The thoracic ribs: each is divided into two parts by a transverse articulation,-a dorsal section, the vertebral 
rib, having all the characters of the last cervical rib ( $\$$ I $4_{4}$, I5), a ventral section, the sternal rib, a straight flat bone, making an obtuse angle with the vertebral rib, and articulating at its lower end with a facet on the sternum.

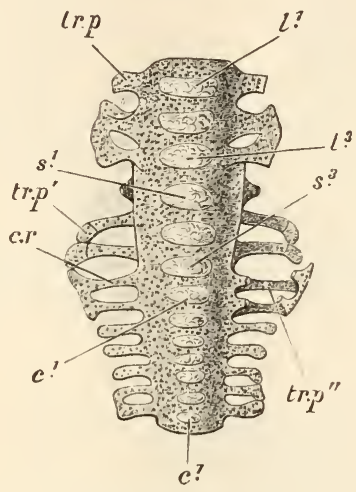

FIG. 46. - Columba livia. The sacrum of a nestling (about fourteen days old), viewed from beneath $(\times 2)$.

On the left side (right of the figure) the first two caudal ribs (c.r) are removed, so as to show the dorsal transverse processes $\left(t r \cdot p^{\prime \prime}\right)$ : the subsequently ossifying membrane which fills up the intervals between the transverse processes is also removed on the same side : the last thoracic vertebra is not shown, not yet having united with the sacrum.

The cartilaginous parts are dotted.

$c . I$, ossified centrum of first, and $c .7$, of seventh caudal vertebra: c.r, first caudal rib: $l .1$, centrum of first, and $l .3$ of third lumbar vertebra: s.I, centrum of first, and s.3 of third sacral vertebra: tr.p, transverse process of first lumbar, tr. $p^{\prime}$, of second sacral, and $t r . p^{\prime \prime}$, of first caudal vertebra.

VI. In the remaining vertebræ entering into the composition of the compound sacrum make out:

26. The two or three lumbar vertebræ (Fig. 46, l. Il. 3), immediately succeeding the fifth thoracic: each has a stout wide body, short strong transverse processes abutting 
against the ilia ( $\$$ I07), rudimentary neural spine, and no hypapophysis.

27. The three or four sacral vertebræ (s.I-s.3), closely resembling the lumbar, but having transverse processes, in the form of thin flat plates passing outwards from the dorsal ends of their neural arches and abutting against the ilia: in the adult, the transverse processes of successive vertebræ are fused together, the original spaces between them being marked only by two rows of small apertures.

28. The first caudal vertebra (c.I), like the sacral, but having, in addition to the lamellar transverse processes springing from its arch, a pair of strong outstanding processes-caudal ribs (c.r)-from its centrum, which also abut against the ilia : a distinct passage is left between the two processes.

29. The remaining ankylosed caudal vertebræ, six in number: in these the centrum becomes progressively smaller, and in all but the first of them (second caudal) there is no distinction between rib and transverse process.

3o. In the young bird the sacrum is a continuous cartilage, in which the constituent vertebræ are only marked by the transverse processes, and by the median ventral ossifications for the centra (Fig. 46).

VII. In the remainder of the vertebral column, note:

3 I. The free caudal vertebræ, each having a short centrum, a well-marked spine, and long, flat, outstanding transverse processes: in the last two there is also a distinct hypapophysis.

32. The pygostyle or ploughshare bone, an irregular vertical plate of bone, with its axis almost at right angles to that of the free caudal vertebræ: it forms the hinder termination of the vertebral column and supports the uropygium ( I 2 I, p. 209). 


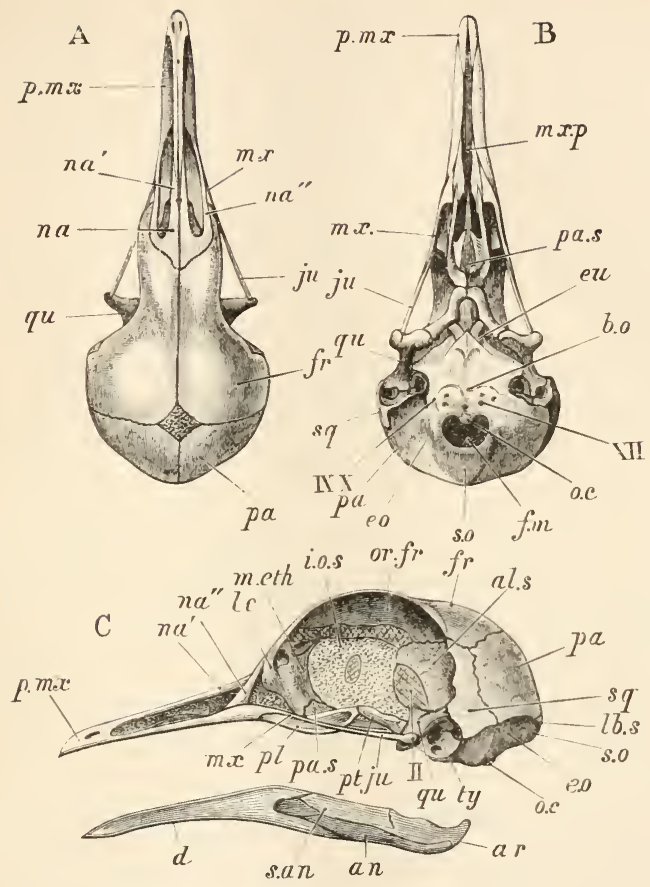

FIG. 47.-Columba livia. The skull of a young bird (about six weeks old). A, from above; $\mathrm{B}$, from beneath; $\mathrm{C}$, from the left side (nat. size).

The cartilaginous parts are distinguished by dotting.

al.s, alisphenoid: an, angular: ar, articular: b.e, basioccipital: $d$, dentary: e.o, exoccipital : eu, aperture of Eustachian tube: f.m, foramen magnum: $f r$, frontal : i.o.s, in erorbital septum : ju, jugal: $l c$, lacrymal : lb.s, lambdoidal suture : m.eth, mesethmoid : $m x$, maxilla : $m x . p$, maxillo-palatine process: $n a, n a^{\prime}, n a^{\prime \prime}$, nasal: o.c, occipital condyle: or $f r$, orbital plate of frontal: $p a$, parietal : pa.s, parasphenoid : $f l$, palatine $: p \cdot m x$, premaxilla: $p t$, pterygoid : $q u$, quadrate : s.an, supra-angular: s.o, supraoccipital : $s q$, squamosal: ty, tympanic cavity : II-XII, foramina for cerebral nerves. 
VIII. In the skull make out :

33. The rounded brain-case, greatly encroached upon in front by the large orbits or cavities for the eyes, and continued in front into the slender tri-radiate beak.

34. The foramen magnum (Fig. 47, f.m) a large rounded aperture in the ventral wall of the hinder end of the brain-case.

35. The occipital condyle (o.c), a small hemispherical elevation on the lowerf or anterior margin of the foramen magnum.

36. The lambdoidal ridge, a transverse ridge, a little above and parallel to the upper border of the foramen magnum : it serves for the attachment of some of the muscles of the neck.

37. In the young bird the foramen magnum is bounded by four distinct bones, one ventral, the basioccipital (b.o), one dorsal, the supraoccipital (s.o), and a pair lateral, the exoccipitals (e.o); the supraoccipital is bounded above by the lambdoidal suture (lb.s), which separates it from the parietals, and is represented in the adult by the lambdoidal ridge.

38. A median longitudinal groove on the dorsal surface of the skull, passing behind into a low ridge which unites with the centre of the lambdoidal ridge : it marks the position of the obliterated suture between the right and left parietals and frontals,

39. In the young bird the parietals $(p a)$ and frontals $(f r)$ form two distinct pairs of bones, the former extending a short distance forward from the lambdoidal suture, while the frontals furnish all the anterior part of the roof of the brain-case, and are continued forwards between the orbits, to each of which the corresponding frontal furnishes a supraorbital ledge. 
40. In the young bird the antero-ventral region of each side of the brain-case is formed by a separate bone, the squamosal $(s q)$, bounded by the frontal and parietal above and behind, by the alisphenoid $(\S 42)$ in front, and by the exoccipital below.

4I. At the posterior margin of each orbit the curved sidewalls of the skull turn sharply inwards, forming a thin plate of bone-the orbital plate-which constitutes at once the anterior wall of the brain-case and the posterior wall of the orbit.

42. In the young bird the orbital plate consists of two parts,-an upper and smaller portion, the orbital plate of the frontal (or.fr), an inturned portion of the latter bone, and a lower and larger portion, forming a distinct bone, the alisphenoid (al.s).

43. The interorbital septum (i.o.s.), a thin vertical plate of bone separating the orbits from one another, and uniting posteriorly with the orbital plates.

44. The anterior part of its interorbital septum consists, in the young bird, of a separate bone, the mesethmoid (m.eth), the posterior part representing the presphenoid.

45. In the dried skull the orbits are placed in communication with one another by two large vacuities, or interorbital fenestræ, situated one above the other, and occurring partly in the orbital plates, partly in the interorbital septum: in the recent state they are filled in by membrane.

46. In the membrane filling the uppermost interorbital fenestra there is a median aperture, the olfactory foramen, for the transmission of the olfactory nerve from the brain : this is continued on each side by a groove, visible in the dried skull, which runs along the dorsal edge of the interorbital septum and, at its anterior end, passes into an 
aperture, which communicates with the nasal cavity, and serves for the passage to that cavity of the olfactory nerve.

47. In the membrane filling the lower interorbital fenestra there is a median aperture, the optic foramen (II), for the transmission of the optic nerves from the brain to the eyes.

48. The tympanic cavity (ty), an irregular depression, just behind the postero-inferior angles of the orbit, its opening looking upwards and forwards: it is bounded by the squamosal above, by the exoccipital behind, and by the basitemporal ( $\$ 50$ ), beneath.

49. The parasphenoid ( $p a . s$ ), or basisphenoidal rostrum, an elongated thickened rod of bone, pointed in front, underlying the interorbital septum with which it is completely ankylosed in the adult: in the young bird it is a separate bone. At its base, it bears, on each side, a small facetted basipterygoid process, for the articulation of the pterygoid ( $\$ 62)$.

50. The basis cranii, or floor of the skull, extending from the lower margin of the foramen magnum to the base of the parasphenoid, and bounded externally by the lower margin of the tympanic cavity: in the young skull the hinder part of the basis cranii is formed, as already seen ( $\$ 37$ ), by the basioccipital ; in front of this is a large median bone, the basisphenoid, underlaid and concealed in the entire skull by the basitemporal, a large flat plate of membrane bone, which extends forwards to the base of the parasphenoid and outwards so as to form the ventral walls of the tympanic cavities.

51. The anterior edge of the basitemporal projects over the base of the rostrum in the form of a delicate lamina of bone, between which and the rostrum is a small space into which the two Eustachian tubes open : a bristle may therefore be passed from this common Eustachian aperture $(\epsilon u)$ into either tympanic cavity ( $\$ 2298,318)$. 
52. The character of the cranial bones as seen in a longitudinal section of the skull : they consist of a dense but very thin outer layer or external table, of a similar inner layer or internal table, and of an intermediate spongy substance or diploë: the latter is especially thick in the anterior part of the frontals and in the basi- and parasphenoid.

53. The hollows on the inner surface of the brain-case for the lodgment of various parts of the brain: the basis cranii is deeply concave from before backwards for the reception of the medulla oblongata (Fig. $58, \mathrm{m.o}$ ) : in the frontal region, or dorsal portion of the brain cavity, is the large cerebral fossa for the reception of the cerebral hemisphere (Fig. $58, c . h$ ); below this and separated from it by a well marked horizontal ridge of bone is the rounded optic fossa for the optic lobe (Fig. $5^{8}, 0 . l$ ); behind and below these two hollows is the cerebellar fossa for the cerebellum (Fig. $5^{8}, c b$ ), limited in front by a ridge-the tentorial ridge-which passes obliquely upwards and backwards from the basis cranii to meet its fellow of the opposite side in the roof of the skull somewhat anterior to the lambdoidal ridge.

54. The auditory capsule, a considerable thickening of the brain-case just external to the foramen magnum, and encroaching upon the optic and cerebellar fossæ: it is limited above by a well marked semicircular ridge, which indicates the position of the anterior semicircular canal, a swelling at its anterior end marking the position of the ampulla of that canal (see figure of cod's auditory organ, p. 123): within the arch of this canal is another ridge, indicating the inner (anterior) portion of the posterior semicircular canal.

The position and relations of the three semicircular canals are best 
seen by cutting away with a penknife the outer table of the cranial bones in the auditory region, and then carefully removing the diploë ; the canals are surrounded by a dense layer of bone, which, isolated in the way described, forms the bony semicircular canals, precisely corresponding in form and position with the contained membranous canals. The anterior canal is considerably larger than the other two; the plane of the posterior canal is at right angles to that of the anterior, and its ampulla is on its lower or outer (= posterior) end; owing to this peculiarity in the position of the posterior canal, it crosses at right angles the horizontal canal, which has the normal position (see description of cod's auditory organ, pp. 122, 123, § I 22 ).

55. In the young bird the main part of the auditory capsule is formed by a single distinct bone, the prootic: two other ossifications are however formed, one upper and posterior, the epiotic, which early fuses with the supraoccipital, another ventral and posterior, the opisthotic, which becomes united with the exoccipital.

56. A little below the swelling for the ampulla of the anterior semicircular canal, on the inner surface of the skull, are two small apertures, for the passage of the seventh (facial) and eighth (auditory) cerebral nerves.

57. Below these apertures, and separated from them by a ridge of bone, is a small oval fossa with an aperture (Ix, $\mathrm{x}$ ) at the bottom of it, which leads directly on to the outer surface of the skull, and serves to transmit the ninth (glossopharyngeal), tenth (vagus), and eleventh (accessory) cerebral nerves.

58. A small aperture within and behind the above is the condyloid foramen (XII); it also leads on to the exterior of the skull and transmits the twelfth (hypoglossal) cerebral nerve.

59. Immediately below the optic fossa are two adjacent apertures which lead on to the anterior surface of the orbital plate, and serve for the passage of the fifth nerve (trigeminal). 
6o. The quadrate (qu), a short, stout, irregular bone, articulating by a double head with two facets in the dorsal region of the tympanic cavity, the upper of the two facets being furnished by the squamosal, the lower by the prootic: from its articulation, the quadrate takes a direction downwards and forwards; its inner border sends off a large pointed ascending process directed upwards, inwards, and forwards, while its distal end forms a transverse cylindroidal articular surface or condyle for the mandible.

6r. The columella (Fig. 48), a small and delicate rod of bone, having its expanded inner end fixed in the fenestra

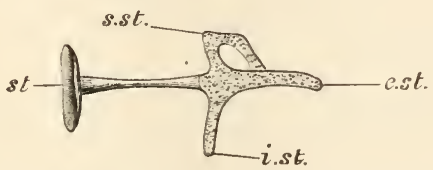

FIG. 48.-Columba livia. The columella auris $(\times 8)$.

The cartilaginous parts are dotted.

e.st, extra-stapedial : i.st, infra-stapedial : s.st, supra-stapedial : st, stapes.

ovalis, a small aperture in the inner wall of the tympanic cavity, while its cartilaginous outer end is fixed, in the entire head, to the inner surface of the tympanic membrane (see § 317, p. 250.)

The columella consists of a slender bony rod, having at its inner end an oval plate of bone, the stapes $(s t)$, and at its outer end a tri-radiate cartilage : the central division of the latter, forming the direct continuation of the stapedial rod, is the extra-stapedial; the second division, situated dorsally, is the supra-stapedial $(s . s t)$; the third, central in position, the infra-stapedial (i.st); the supra- is connected with the extra-stapedial by an oblique bar of cartilage.

62. The pterygoids (Fig. 47, pt), short, stout, paired bones on the base of the skull, each articulating behind 
with a facet on the inner edge of the corresponding quadrate, and passing inwards and forwards to the rostrum, a small facet on its inner surface articulating with the basipterygoid process $(\$ 49)$.

63. The palatines $(p l)$, large paired bones passing forwards from the anterior ends of the pterygoids: their posterior ends are scroll-like, their anterior ends slender and free in the young bird, but ankylosed in the adult with the maxillæ $(\S 67)$.

64. The vomer, a small median bone, lying between the palatines at the anterior end of the parasphenoid.

65. The lacrymal (lc), a considerable bone forming the anterior wall of each orbit, and articulating above with the corresponding frontal.

66. The premaxillæ $(p m x)$, paired bones forming the greater part of the beak, and completely ankylosed with one another in the adult: the anterior or free ends of the united bones form the strong pointed extremity of the beak, from which three processes are given off on each side : the first of these, the nasal process, passes upwards and backwards on the dorsal aspect of the beak, fused with its fellow of the opposite side, to join the anterior edge of the frontals; the palatine process extends backwards and slightly outwards on the ventral aspect of the beak, and is fused in the adult with the corresponding palatine; and the maxillary process passes backwards and outwards, external to the palatine process, forming the edge of the beak, and uniting posteriorly with the maxilla.

67. The maxillæ $(m x)$, continuing backwards the maxillary processes of the premaxillæ, to which they are ankylosed in the adult: anteriorly the inner edge of each is produced into an inwardly-directed, spongy mass of bone, the maxillopalatine process $(m x p)$, which is ankylosed 
in the adult to the maxilla: the remainder of the maxilla forms a very slender rod of bone.

68. The jugal $(j u)$, a slender bone, continued backwards from the hinder end of the maxilla, with which it is ankylosed in the adult.

69. The quadrato-jugal, a similar bone to the jugal, to which it is fused in the adult, and from which it is continued backwards, its posterior end being attached by ligament to the outer edge of the quadrate just above its condyle.

70. The nasal (na), a long, forked bone, fitting against the anterior edge of the frontal, and having a short external process $\left(n a^{\prime \prime}\right)$ which extends obliquely downwards and forwards to the maxilla, and a longer anterior process $\left(n a^{\prime}\right)$ which passes forwards external and parallel to the nasal process of the premaxilla.

$7 \mathrm{I}$. The anterior nasal fossæ, large vacuities one on ach side of the beak, bounded above by the internal processes of the nasals and nasal processes of the premaxillæ, below by the maxillæe, and behind by the external processes of the nasals.

72. In the fresh state, the mesethmoid ( $\$ 44)$ is continued forwards to the end of the beak by a plate of cartilage, the septum nasi, which forms a median vertical partition between the nasal cavities: it is produced on each side into scroll-like masses of cartilage, the turbinals.

IX. In the lower jaw note :

73. The complete fusion, in the adult, of its two halves or rami in a short median symphysis.

74. The posterior half of each ramus can be separated as a distinct bone which fits by a jagged edge into the corresponding anterior portion: in the young bird, this proximal portion of the mandible consists of three separate 
bones; a stout irregular articular (ar), furnishing the articular cavity for the quadrate, and continued forwards into a slender Meckel's cartilage ; a splint-like bone, the angular $(a n)$, covering the ventral edge of the articular and Meckel's cartilage; and the supra-angular (s.an) similarly related to their upper edge.

75. From the inner face of the distal portion of each ramus a thin splint, the splenial, can be separated even in the adult: the remainder of the distal portion is the dentary (d).

$\mathrm{X}$. Observe in the hyoidean apparatus :

76. The body of the hyoid, consisting of three distinct portions: the first or glosso-hyal, is cartilaginous and arrowhead-shaped, the second or basi-hyal, is a short stout bone, articulating in front with the glosso-hyal, and behind separated by a cartilaginous interval from the third portion or basi-branchial, which consists of a slender bony rod with a free cartilaginous extremity.

77. The anterior cornua, short, paired, cartilaginous rods, proceeding backwards and outwards from the posterior end of the glosso-hyal, like the barbs of the arrowhead.

78. The posterior cornua, slender paired rods, springing from the cartilaginous junction between the basi-hyal and basi-branchial, and curving backwards, outwards, and upwards: each consists, first, of a proximal or ventral portion, a slender bony rod, the cerato-branchial; this is connected at its distal end with a similar rod, the epibranchial, which terminates distally in a slender pointed cartilage.

XI. Make out the characters of the sternum as follows :

79. The body or corpus sterni, a horizontal boatshaped plate of bone, its right and left halves inclined to 
one another so as to include an obtuse dihedral angle, the transverse sternal angle.

8o. The middle xiphoid process, a narrow, flat, median plate, forming the hinder end of the corpus sterni.

8r. The internal xiphoid processes, flat paired plates proceeding outwards and backwards from the corpus sterni at the point of origin of the middle xiphoid process, with the posterior border of which they are connected by narrow bridges of bone: two large fenestræ, covered in the recent state by membrane, are thus enclosed between the middle and internal xiphoid processes.

82. The external xiphoid processes, similar to but much larger than the internal xiphoids, and passing backwards and outwards from the lateral borders of the corpus sterni, about half way between its anterior end and the origin of the internal xiphoids.

$8_{3}$. The costal border, or that part of the outer edge of the sternum lying anterior to the external xiphoid process : it is produced in front into a forwardly directed costal process, and presents four facets for the articulation of the sternal ribs.

84. The manubrium or rostrum sterni, a small blunt median prolongation of the anterior border of the corpus sterni.

85. The pneumatic aperture, a small aperture on the dorsal surface of the corpus sterni about a centimetre behind its anterior border: through it the interclavicular air-sac ( $\S \mathrm{r} 64$, p. 218 ) communicates with air spaces in the bone: these spaces can be seen as a delicate transparent network by holding up the sternum to the light.

86. The coracoid grooves, deep paired fossæ on the anterior border of the body of the sternum, extending 
from near the middle line immediately ventral to the rostrum, outwards and slightly backwards to the costal processes.

87. The keel or carina sterni, a median ventral plate of bone, running along the whole middle ventral line of the corpus sterni from the base of the rostrum to the middle xiphoid process : its depth is about equal to the breadth of the body of the sternum.

88. In a bird about three or four weeks old, the sternum is formed of three distinct bones : one median, the lophosteon, ossifying the central part of the body and the keel, and an external pair, the pleurostea, forming the lateral parts. $^{1}$

XII. In the shoulder girdle note :

89. The coracoids, paired rod-like bones, articulating by their laterally expanded ventral ends with the coracoid grooves of the sternum, and passing forwards, upwards, and slightly outwards : each coracoid is somewhat depressed from above downwards, and presents at its upper"end a thickened head : immediately below the head on the dorsal side of the bone are two facets for the articulation of the scapula: on the inner border at about the same level is a facet for the head of the furcula ( $\$ 9 \mathrm{I}$ ), and on the outer border a large demi-facet which forms the anterior half of the glenoid cavity for the articulation of the wing: towards the inner border of the upper end of the coracoid is a groove passing into a canal which opens on the upper surface of the head between the scapular facets, and serves for the passage of the tendon of the subclavius ( $\$ 326$, p. 252): this cavity, being completed by the articulation of the scapula $(\S 90)$ and the furcula ( $(9 \mathrm{I})$ with the coracoid, is known as the foramen triosseum.

1 In the young chick a separate pair of ossifications, the metostea, gives rise to the internal and external xiphoid processes. 
90. The scapula, a sabre-shaped bone, articulated immovably to the corresponding coracoid, so as to make with it a somewhat acute angle-the coraco-scapular angle, and passing backwards over the ribs: its proximal or anterior end is expanded, and presents two facets for articulation with the coracoid, and, externally, a large demifacet which completes the glenoid cavity.

9I. The furcula, or "merrythought," a V-shaped bone, articulating by a facet on the outer surface of each of its extremities with the facet already seen on the corresponding coracoid, and passing backwards and downwards, its angle being connected by ligament with the carina sterni.

92. In the young bird, the furcula consists of separate paired bones, the clavicles, with a small median ossification, the interclavicle.

XIII. Note in the fore-limb :

93. The general composition of the limb : it consists of a proximal division, the brachium, containing a single bone, the humerus; a middle division, the antibrachium, containing two bones, a larger, the ulna, and a smaller, the radius ; and a distal division, the manus.

94. The position of the parts : in the position of rest the three divisions are bent upon one another in the form of a $\mathbf{Z}$; extended for flight, they are almost in the same straight line: in the latter case the limb presents a dorsal and a ventral surface, pre-axial or anterior, and post-axial or posterior, borders; the radius is then seen to be preaxial, and the ulna post-axial. In the position of rest, the humerus extends backwards from its articulation with the shoulder girdle, undergoing at the same time a slight rotation, so that its dorsal surface looks inwards and upwards, and its pre-axial border outwards and upwards; the antibrachium has its dorsal surface directed outward 
and its pre-axial border upwards; in the manus the dorsal surface looks upwards and the pre-axial border downwards and forwards.

95. The humerus, an elongated bone, consisting of a cylindrical shaft and of two extremities: the proximal extremity presents a rounded elevation or head for articulation with the glenoid cavity, a triangular process, the greater or radial tuberosity on the pre-axial side, and an irregular elevation, the lesser or ulnar tuberosity on the postaxial side: on the distal surface of the latter is a deep excavation leading to the pneumatic foramen which communicates with a large air-cavity in the shaft of the bone. The distal end of the humerus has a pulley-like surface or trochlea, with two raised articular surfaces, one - the radial tubercle-pre-axial and set obliquely, the other-the ulnar tubercle-post-axial and nearly at right angles to the first: just external and proximal to the radial tubercle is a small roughened elevation, the radial condyle ; the ulnar condyle is similarly related to the ulnar tubercle.

96. The radius, a straight slender bone, articulating by its discoid concave proximal extremity or head with the radial tubercle of the humerus: its distal end bears an oblique, elongated convexity for articulation with the carpus; it is not pneumatic.

97. The ulna, a curved bone, having its concave side towards the radius which it considerably exceeds in thickness : its proximal end presents a concave surface, the sigmoid cavity, set obliquely to the shaft for articulation with the humerus, and is produced beyond this facet, postaxially, into a short, blunt process, the olecranon; a short process is also given off from the dorsal and pre-axial region which partly embraces the head of the radius. 
98. The carpus, or proximal division of the manus, consisting of two short bones, the smaller (radiale, Fig. 49, $c p . \mathrm{I})$ articulating with the radius, the larger (ulnare, $c p .2$ ) with the ulna.

99. The metacarpus or middle division of the manus, consisting of $(a)$ a large cylindrical bone, the second metacarpal $(m c p .2)$; (b) a slender curved bone, the third metacarpal (mcp.3), on the post-axial side of the second, to which it is ankylosed at its extremities, its shaft remaining

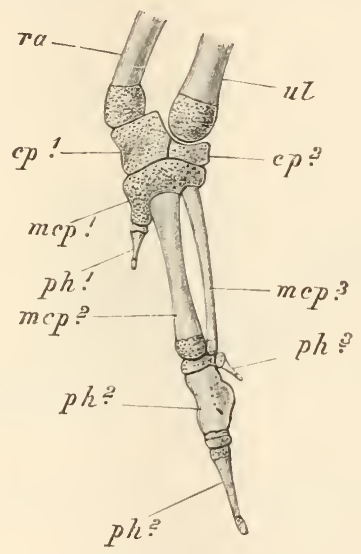

FIG. 49. - Columba livia. The left manus of a nestling (about fourteen days old), viewed from the outer (dorsal) surface $(\times 2)$.

The cartilaginous parts are dotted.

$c p . \mathrm{I}$, radial, and $c p .2$, ulnar carpal : $m c p . \mathrm{I}$, first, $m c p .2$, second, and $m c p .3$, third, metacarpal: ph. I, phalanx of pollex: ph.2, proximal, ancl $p h .2^{\prime}$, distal phalanx of second digit : $p h .3$, phalanx of third digit : $r a$, radius : $u l$, ulna.

free; $(c)$ the first metacarpal (mcp.r), forming merely a projecting process on the pre-axial border of the proximal end of the second.

Iо०. The phalanges, forming the distal division of the manus: one pointed bone (ph.r), attached to the 
rudimentary first metacarpal is the single phalanx of the first digit or pollex: the second digit has two phalanges, the proximal one $(p h .2)$ being flattened and very thin on its post-axial side, the distal one ( $\left.p h .2^{\prime}\right)$ pointed and concave dorsally: the third metacarpal bears a single pointed phalanx (ph.3).

ror. In the young bird the metacarpals are distinct bones with cartilaginous epiphyses.

XIV. Make out the characters of the pelvis or hip girdle as follows :

I02. It consists of two paired bones, the ossa innominata (Fig. 50), more or less extensively ankylosed to

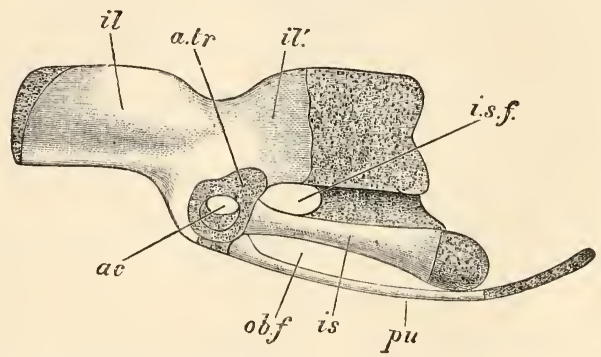

FIG. 50.-Columba livia. Left os innominatum of a nestling (fourteen days), viewed from without $(\times 2)$.

The cartilage is distinguished by dotting.

$a c$, acetabulum: a.tr, anti-trochanter: $i l$, pre-, and $i l^{\prime}$, postacetabular portion of ilium : is, ischium : i.s.f, ilio-sciatic foramen : $o b . f$, obturator fissure : pu, pubis.

the sacrum in the adult, but easily separable in the young bird.

I03. The acetabulum (Fig. 48, ac), a rounded articular cavity on the outer surface of each os innominatum, perforated by an aperture, and serving for the articulation of the leg: its postero-dorsal region is prolonged into a distinct facet, the anti-trochanter (a.tr). 
104. The ilio-sciatic foramen (i.s.f), a large aperture immediately posterior to the acetabulum.

105. The pubis $(p u)$, a slender bony rod, forming the ventral part of the acetabulum and the ventral border of the post-acetabular portion of the innominate bone: it is separated from the rest of the bone by a long cleft, the obturator fissure (ob.f).

106. The ischium (is), consisting of that part of the post-acetabular portion of the innominate bone which lies above the obturator fissure and below and behind the iliosciatic foramen: at the acetabulum it is ankylosed with the anterior end of the pubis.

107. The rest of the os innominatum is formed by the ilium $\left(i l, i l^{\prime}\right)$, which consists of pre- and post-acetabular portions, the former (it) ankylosed at the ventral boundary of the acetabulum with the pubis, the latter $\left(i l^{\prime}\right)$ ankylosed with the ischium in the postero-dorsal region of the acetabulum, as well as behind the ilio-sciatic foramen.

I08. In the young bird the os innominatum consists of a distinct mass of cartilage of the same shape as the adult bone, in which the ilium, ischium, and pubis are formed as separate ossifications, which unite with one another at a later period.

$\mathrm{XV}$. In the leg make out :

ro9. Its general composition: it consists of a proximal division, containing a single bone, the femur; a middle division, the crus or shank, containing a large bone, the tibio-tarsus, and a small and imperfect one, the fibula; and a distal division, the pes, formed of a cylindrical bone, the tarso-metatarsus, to which four digits are attached.

Iro. The position of the parts: the femur is directed downwards and forwards from its articulation with the 
pelvis; the crus is directed downwards and slightly backwards, the tarso-metatarsus downwards and slightly forwards, three of the digits are directed forwards and rest with their whole lower surface on the ground, while the remaining toe -the hallux or first digit-is directed backwards, and slightly raised from the ground at its proximal end.

I I I. If the leg is extended outwards at right angles to the body it is seen to present, like the fore-limb, dorsal and vental surfaces and pre-axial and post-axial borders : in the natural position of the parts the pre-axial border is internal throughout the whole limb; the primitively dorsal surface looks upwards and forwards in the femur, forwards and slightly downwards in the crus, forwards and slightly upwards in the tarso-metatarsus, and upwards in the digits.

I I 2. 'The femur, a cylindrical bone consisting of shaft and extremities : the proximal extremity is produced on the pre-axial (inner) side into a rounded elevation or head, the axis of which is at right angles to that of the shaft, and which serves for articulation with the acetabulum: postaxially is a large irregular elevation, the great trochanter, and between it and the head on the actual proximal end of the bone is a facet which works against the anti-trochanter ( $\S$ I03). The distal end of the femur is pulley-like, and formed of two condyles; of these the outer or postaxial condyle is deeply grooved in its ventral region, thus furnishing the fibular fossa for the articulation of the fibula; the rest of the outer and the whole of the inner condyle articulates with the tibio-tarsus.

II3. The tibio-tarsus, a cylindrical bone about half as long again as the femur: its shaft, together with the proximal extremity, corresponds to the tibia ; its distal extremity represents the proximal portion of the tarsus, and therefore really belongs to the pes. The proximal extremity bears 
two slightly concave articular surfaces for the condyles of the femur, and is produced in front (dorsally) into a prominent cnemial crest divided into two diverging plates. The distal extremity is pulley shaped, and its transverse axis is inclined at an angle to that of the proximal extremity: the two condyles of which the distal pulley is formed are more prominent on the anterior (dorsal) than on the posterior face of the bone, thus differing from the very similar condyles of the femur.

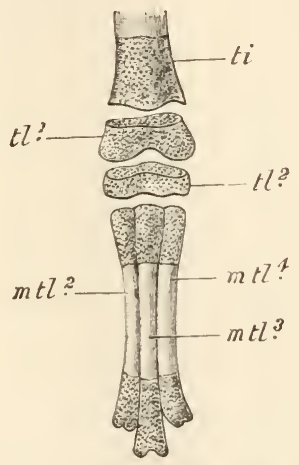

FIG. 51.-Columba livia. Left tarso-metatarsus of an unhatched einbryo, with the distal end of the tibio-tarsus, viewed from the anterior (dorsal) face $(\times 5)$.

The tarsal cartilages $(t l .1, t l .2)$ are represented as separated from the tibia and metatarsus respectively.

The cartilaginous parts are dotted.

$m ! l .2$, second, $m t l .3$, third, and $m t l .4$, fourth metatarsal: $t i$, tibia: tl.I, proximal tarsal cartilage, afterwards fusing with tibia: $t l .2$, distal tarsal cartilage, afterwards fusing with metatarsus.

I 4. In a pigeon embryo towards the end of incubation, the distal end of the tibio-tarsus is a separate cartilage, the proximal tarsal cartilage (Fig. 5 I, tl. I) : its separateness in the cartilaginous state distinguishes it from an epiphysis, which is a distinct ossification in a continuous cartilage. 
II5. The patella, a small nodule of bone in front of the knee-joint, connected by ligament with the femur and tibio-tarsus.

I 6. The fibula, a slender bony rod attached to the outer (post-axial) side of the tibio-tarsus : its proximal end articulates with the fibular fossa in the outer condyle of the femur: distally it undergoes a gradual diminution in diameter and finally fuses with the tibio-tarsus.

I 7 . The tarso-metarsus, a cylindrical bone articulated to the distal end of the tibio-tarsus, its proximal end bearing two concave surfaces for the condyles of the latter bone: behind (ventrally) it is produced at its proximal end into a strong crest, on either side of which is a small foramen passing through to the somewhat concave anterior face of the bone: its distal end is trifurcate, the middle division being the longest, and each bearing a pulley-like surface for the articulation of one of the toes. That part of the tarso-metatarsus which lies above (proximal to) the two foramina represents the distal tarsals : in the remainder of the bone, the two foramina and trifurcate distal ends indicate a division into three longitudinal parts, the second, third, and fourth metatarsals. The joint between the tibiotarsus and tarso-metatarsus is conveniently known as the mesotarsal articulation, being between the proximal and distal tarsals.

I 8 . In an embryo towards the end of incubation, the proximal extremity of the future tarso-metatarsus is a separate cartilage, the distal tarsal cartilage (Fig. 5 I, tl.2) and the remainder is formed of three separate long bones, (metatarsals, $m t l .2, m t l .3, m t l .4$ ), in close contact save at their distal ends.

I 9. The hallux, or first pre-axial digit, consisting of a separate metatarsal, united by ligament to the inner border 
of the tarso-metatarsus near its distal end, and of two phalanges, a proximal and a distal, the latter modified for the support of a claw.

I 20. The three forwardly directed toes, representing the second, third, and fourth digits, and having their metatarsals united to form the tarso-metatarsus: the second digit, or innermost of the three, consists of three phalanges, the next of four, and the fourth or outermost of five, the distal phalanx in each case being modified for the support of a claw.

\section{B.-DIRECTIONS FOR DISSECTION.}

XVI. Either pluck the feathers from one side of the bird, leaving the other side untouched, or better, have two birds for comparison, one entire, the other plucked: note the following external characters :

I 2 I. The division of the body into head, neck, trunk, and limbs: the relatively great length of the neck: the absence of a true tail, the body terminating posteriorly in a short obtusely conical process, the rump or uropygium.

I22. The great influence of the external covering of contour feathers or pennæ in determining the contour of the body, the form of the plucked bird being altogether different from that of the entire one.

I 23. The filoplumes, small, hair-like feathers, situated between the contour feathers, and exposed by the removal of the latter in the plucked bird.

I24. The rounded form of the cranial portion of the head, and the production of the facial portion into a 
beak, devoid of feathers but covered with a strong, horny sheath, except at the base, where there is on each side a tumid naked area of skin, the cere.

125. The mouth, bounded above and below by the upper and lower divisions of the beak (upper and lower mandibles of ornithologists).

126. The nostrils, or external nares, oblique, slitlike, paired apertures immediately in front of the cere.

x 27. The eyes, situated a little posterior to the angle of the mouth: each is provided with an upper and a lower eyelid, both devoid of feathers, and with a nictitating membrane, which is easily found in the anterior angle of the eye, and can be pulled backwards over the eye as a whitish, semi-transparent membrane.

128. The auditory aperture, situated a short distance below and behind the eye, of a rounded form, and leading into a canal, the external auditory meatus : in the entire bird the auditory aperture is quite concealed by the feathers.

I29. The flaccid condition of the skin about the neck, due to the more or less empty condition of the crop (§ $\mathrm{r} 50$ ).

I30. The breast of the bird, formed by the muscles covering the sternum : the keel of the latter $(\$ 87)$ can be felt as a median bony ridge extending from a short distance behind the root of the neck to within $1 \frac{1}{2}$ inches of the posterior extremity of the body.

I3 I. The soft, ventral, abdominal walls behind the posterior edge of the sternum, bounded externally and posteriorly by the pubes ( $(105)$.

132. The vent, or cloacal aperture, a considerable transverse cleft with tumid lips, situated on the ventral surface of the hinder end of the trunk. 
I33. On the dorsal surface of the conical posterior extremity of the trunk is a small papilla on the apex of which opens the duct of the uropygial or oil.gland ( $\$ 296$, p. 247).

134. The division of the fore-limb or wing into a proximal portion, the brachium or upper arm, a middle portion, the antibrachium or fore-arm, and a distal portion, the manus or hand, the three divisions being bent upon one another when at rest in the form of a $\mathbf{Z}:$ the absence of distinct digits, except in the case of the pollex or thumb, which forms a small projection on the pre-axial (\$ 94) border of the manus at its proximal end.

I35. The alar membrane, a fold of skin, extending between the proximal end of the fore-arm and that of the hand on the pre-axial side.

136. The division of the hind-limb into a proximal portion, the thigh or femur, a middle portion, the shank or crus, and a distal division, the foot or pes; the latter consists of a proximai stem, the tarso-metatarsus, and of four digits, the first (pre-axial, $\S$ II I) of which is directed backwards, and all of which terminate in strong claws.

I37. The arrangement of the feathers on the neck and trunk: they are not continuous, but form a number of feather-tracts or pterylæ, separated by featherless spaces or apteria: the former being indicated in the plucked bird by the scars left by the removal of the feathers. ${ }^{1}$ Along the dorsal surface runs the spinal tract, the cervical portion of which is particularly well marked,

1 The pterylosis, or feather arrangement, is better made out in the sparrow than in the pigeon, as in the latter the feather tracts tend to run together: in the sparrow, on the contrary, the spinal tract is almost linear, broadening out only in the lumbar region; the ventral tract consists of two very narrow bands separater by an inferior space occupying nearly the whole of the pectoral region; the lateral spaces are also very large. 
and bifurcated posteriorly: on the veniral surface is the ventral tract, divided in the region of the trunk into two lateral portions separated from one another by the median inferior space : throughout the greater part of its extent the ventral is separated from the spinal tract by the lateral spaces, one on either side of the trunk and neck.

I 38. The arrangement of the tail feathers: attached along the posterior edge of the rump are twelve large rectrices or tail quills, the position of which is indicated by well marked scars in the plucked bird: the proximal ends of the rectrices are covered both above and below by smaller feathers, the tail coverts, attached to the upper and under surface of the rump.

139. The arrangement of the feathers on the wing: along the post-axial edge of the antibrachium and manus is attached a row of about twenty large remiges or wing quills, of which those attached to the manus are the primaries, those attached to the antibrachium the secondaries: their position is clearly indicated in the plucked bird by large scars. Both on the upper and under surface of the wing the remiges are largely covered by the upper and under wing coverts, which also form the entire feathery covering of the upper arm : on the dorsal surface of the latter the feathers are arranged in a well marked humeral tract: the secondary upper wing coverts spring from an alar tract. A special tuft of feathers springs from the pollex, and constitutes the ala spuria or bastard wing.

I 40. The arrangement of the feathers on the leg: there is a well defined femoral tract on the dorsal surface of the thigh, and a weak crural tract on the shank.

I 4I. The absence of feathers on the pes, which is covered instead by horny scales: these are especially large on the 
anterior (dorsal) surface of the tarso-metatarsus and on the dorsal surface of the toes.

XVII. ${ }^{1}$ In one of the large quill feathers make out the following points :

I42. The stem, or scapus, consisting of a proximal portion, the tube or calamus, which is cylindrical and hollow or nearly so, and of a distal portion, the shaft or rachis, which is somewhat quadrate in section, grooved on its ventral ${ }^{2}$ side, and formed internally of a white, pith-like substance.

I43. The barbs, delicate tapering processes, flattened in a direction at right angles to the long axis of the feather and attached in two rows one on each side of the rachis: they constitute, taken together, the vane or vexillum of the feather.

I44. The inferior umbilicus, a small aperture at the proximal end of the calamus, by which the feather-papilla enters the latter.

I45. The superior umbilicus, a small oblique aperture at the junction of the calamus and rachis, putting the cavity of the former in communication with the exterior.

XVIII. Cut off a small piece of the vane and examine it under a moderately high power of the microscope, taking care to distinguish between the dorsal and ventral surfaces, and the proximal and distal ends of the piece. It is advisable to soak the fragment for a short time in alcohol, so as to remove the air, and then to examine in glycerine. Note :

146. The barbules, small pointed processes, at ached obliquely in two rows one on each side of the barbs, to which they are related in the same way as are the latter, to the rachis: they are so disposed that the

1 It will probably be found convenient, in practice, to omit Sections XVII-XIX until the dissection of the soft parts is completed, and to proceed from § I4I to Section XX., p. 2 I4.

2 That is, the lower side in the case of the remiges and rectrices, or the inner side in the case of the ordinary contcur feathers. 
distal barbules of any given barb overlap and cross at an angle the proximal barbules of the barb next in advance.

I47. The hooklets, minute curved processes on the distal barbules, by means of which the latter are hooked on to the proximal barbules of the next succeeding barb : the proximal barbules themselves are devoid of hooklets.

XIX. Pluck out a filoplume and examine it in the same way: note :-

I48. The delicate stem, showing no distinction between calamus and rachis.

I49. The rudimentary vane, consisting of a few barbs with simple disunited barbules.

$\mathrm{XX}$. In the plucked bird, thrust a blowpipe into the mouth and loop a string round the base of the beak immediately behind the cere, tightening it just enough to hold the blowpipe in place and prevent the escape of air: inflate until the neck and abdomen are greatly swelled owing to the distension of the crop ( $\S$ I 50$)$ and the air-sacs (\$ I 59): withdraw the blowpipe, at the same time tightening the ligature: then knot the latter to prevent its loosening.

By this method both crop and air-sacs are inflated simultaneously without injury to any of the structures to be afterwards examined: if in the course of dissection one or other should collapse -which is certain to happen sooner or later-cut across the trachea ( I $_{5} \mathrm{I}$ ) and inflate the crop from its anterior and the air-sacs from its posterior end, ligaturing as before. ${ }^{1}$

1 If it is desired to make a careful dissection of the air-sacs, dissect away the muscles from the humerus, and cut that bone across a short distance from its distal end : slip over the cut end a piece of indiarubber tubing, to the other end of which is fixed a short glass tube: fix the latter in a vertical position: make an incision into the trachea and insert a cannula directed backwards, tying it securely : inject slowly with 
Make a longitudinal incision through the skin over the keel of the sternum, and continue the incision backwards to the vent and forwards to the throat : dissect away the skin from the whole ventral surface of the body and reflect it right and left, being especially careful in removing it from the crop, to which it is closely adherent. Note :-

150. The œsophagus or gullet, a wide thin-walled tube, lying along the ventral aspect of the neck, and enlarging posteriorly into a bilobed dilatation, the crop: both gullet and crop are now unnaturally distended.

I5r. The trachea, a narrow tube, surrounded: at short intervals with bony rings, which give it an annulated appearance : it lies on the ventral side of the anterior end of the gullet, but soon crosses to the left and passes to the dorsal side of the crop.

I 52. The jugular veins, large vessels, usually gorged with blood in a freshly killed bird, running one on each side of the neck, dorsal to the crop: each jugular is seen to receive feeders from the gullet and crop, as well as from a more or less extensive venous plexus in the skin of the neck.

153. The pectoralis major, a large muscle forming the greater part of each side of the breast: its fibres are readily seen to arise from the carina sterni and from the clavicle, and to pass forwards and outwards, converging as they go, to the ventral aspect of the humerus (see $\S 327$, p. 252).

I54. The abdominal muscles, forming the soft fleshy

plaster of Paris or gelatine (see p. $48, \S$ I 62) until the injecting fluid rises in the glass tuhe. When the plaster or gelatine has set, remove the indiarubber tube from the humerus, and proceed as directed in the succeeding paragraphs. 
ventral walls of the body between the posterior edge of the sternum and the pubes.

I55. The rectus abdominis, a narrow paired muscle formed of longitudinal fibres, forming with its fellow the median portion of the ventral abdominal wall : it extends from the posterior border of the sternum to the pubis; its posterior portion is tendinous.

156. The obliquus externus, a broad sheet of muscle forming the lateral portion of the abdominal wall, and extending, in fiont, over the posterior ribs : the muscle proper, consisting of fibres directed downwards and backwards, only extends about half way between the posterior border of the sternum and the pubis, but is continued backwards to the latter bone by a strong sheet of connective tissue or aponeurosis.

157. The obliquus internus, situated beneath the external oblique, which must be removed to show it: it is almost co-extensive with the external ollique, and like it, its posterior portion is aponeurotic: its fibres are nearly longitudinal in direction.

158. The transversus abdominis, lying beneath the internal oblique and formed of transversely directed fibres, which are seen through the posterior or aponeurotic portions of the obliqui.

XXI. Make a median longitudinal incision through the abdominal walls from the posterior edge of the sternum to the vent: from the anterior end of this cut make transverse incisions along the posterior border of the sternum, taking care not to cut too deeply and so injure the air-sacs (§ I59): carefully reflect the flaps of muscle thus separated, and note:

I59. The (abdominal) posterior air-sacs, one on either side of the body-cavity or cœlome now exposed : they are transparent thin-walled sacs, unnaturally distended with air, and largely concealed at present by the great omentum ( $(\mathrm{r} 6 \mathrm{r})$ and the viscera ( $\mathrm{I} 62)$.

160. The posterior-intermediate air-sacs, lying one on each side immediately in front of the corresponding 
abdominal sacs : to see them the edge of the sternum must be slightly raised.

I6r. The great omentum, a sheet of membrane, loaded with fat, which covers the contents of the abdomen; by carefully lifting it the posterior air-sacs will be better displayed.

162. Some of the abdominal viscera (liver, intestine, \&c.) can also be seen at this stage of the dissection, but are best examined later ( $\$$ i 7 I, \& $\mathcal{S}$.).

XXII. Carefully dissect away the crop from the anterior surface of the sternum and pectoral muscles, taking care not to injure the interclavicular and prebronchial air-sacs ( $\left.\$_{1} 6_{4}\right)$ : make a longitudinal incision through either of the great pectoral muscles close to the carina sterni,-the incision should be about half an inch deep at the anterior end of the carina, gradually diminishing in depth as it is continued backwards, and must be made with considerable care, so as not to injure the subclavius muscle (\$ I66): continue the incision forwards, gradually separating the pectoralis major from the furcula, and from the posterior end of the incision continue it outwards so as to separate the muscle from the corpus sterni : when these attachments, together constituting the origin of the pectoralis, are cut through, the muscle will come away quite easily from the underlying parts: reflect it outwards, taking care not to injure the pectoral vessels ( $\S$ I 68 ) or the axillary air-sacs ( $\left.\$ \mathrm{I}_{5}\right)$, and note :

I63. The distal portion of the gullet, passing backwards from the crop, of which it looks like a middle lobe, to reach the body-cavity. 
I64. The interclavicular air-sac, situated at the bottom of the pit bounded at the sides by the furcula and pectoral muscles, in front by the crop and by the carina : it is distinctly bilobed, being formed by the fusion of two sub-bronchial air-sacs, and extending on either side of the gullet: immediately dorsal to each of its lobes is one of the paired prebronchial air-sacs.

165. A prolongation of the interclavicular air-sac on each side into the corresponding axilla, placed just external to the coracoid, and covered immediately by the pectoralis major.

166. The subclavius or pectoralis secundus, a broad flat sheet of muscle, arising from the dorsal portions of the keel and median portions of the body of the sternum: it is a bi-pinnate muscle, that is, is divided longitudinally by a tendinous band to which the fibres of either side converge : anteriorly it is seen to pass forwards and outwards between the furcula and the axillary prolongation of the interclavicular air-sac.

I67. The coraco-brachialis longus, a small muscle lying external to the anterior portion of the subclavius, and passing forwards and outwards dorsal to (beneath in the present position) the axillary portion of the interclavicular air-sac.

I68. The pectoral arteries and veins, large vessels distributed to the pectoral muscles, and, at present, preventing further reflection of the pectoralis major. ${ }^{1}$

1 The injection of the pigeon's vascular system is best performed in the following way. As soon as the bird, killed with chloroform or potassic cyanide, is dead, pluck the breast, expose the pectoral vessels of one side as directed in Section XXII., cut through these vessels as near as possible to the reflected pectoralis major, and allow to bleed. All this should be done with the greatest rapidity, as birds' blood coagulates very quickly, and it is essential to success to allow as much as possible 
XXIII. Ligature the pectoral vessels, if the subject is not injected: cut through the origin of the subclavius and reflect it: cut away the greater part of both pectoralis and subclavius, leaving a recognisable portion of the anterior end of each still attached to the upper arm: do the same on the other side, thus exposing the whole sternum as well as the greater part of the coracoids and furcula: make a longitudinal cut, with strong scissors, through one side of the sternum parallel and close to the keel, and extending to about the middle of the latter : gradually cut or break away the posterior part of the body of the sternum external to this incision until the following structures can be seen :

I69. The anterior-intermediate air-sac, immediately in front of the corresponding posterior-intermediate ( $\S \mathrm{I} 60)$.

r 70. The falciform ligament, a median vertical sheet of peritoneum, attached along the middle line of the sternum, and passing dorsalwards among the viscera ( $\left(\mathrm{I} 8_{5}\right)$.

to escape. Remove the corpus sterni on the same side, so as to expose the heart and see the origin of the pectoral vessels ( $\S$ I $6 S$ ) : insert a cannula into the pectoral artery through the incision already made, tie securely, and inject towards the heart: in this way the whole of the arterial system is filled. The systemic veins may be injected from the pectoral vein, but better results are obtained by injecting from the coccygeo-mesenteric vein ( $\$ 197)$, the cannula being injected backwards, or towards the renal portal veins : the severed pectoral vein should first be tied or clamped with bull-dog forceps. It will probably be found necessary to inject the precavals $(\$ \S 243,245)$ and their feeders separately : this is best done by making an incision in one of the jugulars (preferably that of the side on which the pectorals have already been cut) near its proximal end, and injecting forwards. The portal system is best injected from the coccygeo-mesenteric vein (\$ I97), the cannula being directed forwards. 
In the uninjured state the falciform ligament is contirued backwards attached to the middle ventral line of the abdomen.

XXIV. Cut through the other side of the sternum in the same way: lift up the keel, and gradually extend both incisions forwards, using bone-forceps when necessary, until the whole of the central part of the sternum is removed: cut through each coracoid at about its middle and remove its ventral portion: also remove the furcula, and enough of the lateral regions of the sternum, with the sternal ribs, to bring all the viscera into view : note, without disturbing anything :

I7 I. The reddish-brown liver, occupying the middle region of the cclome, and consisting of a large right and a small left lobe.

I72. The heart, very large in proportion to the size of the animal, lying in the middle line in front of the liver, and enclosed in a thin membrane, the pericardium.

r 73. The duodenum, a pinkish, U-shaped loop of intestine, passing from beneath the edge of the right lobe of the liver to the posterior end of the colome, and consisting of a left or proximal and a right or distal limb.

r74. The pancreas, a compact reddish gland, lying between the two limbs of the duodenal loop.

I75. One or two loops of the ileum (the chief part of the small intestine) are seen towards the right of the cœlome.

I76. The gizzard, a hard oval body, on the left side of the body, just posterior to and partly concealed by the liver.

r77. The distal end of the trachea, curving round the left side of the crop to reach the ventral aspect of the gullet, and almost immediately dividing into the two 
bronchi, which pass outwards and backwards to the lungs ( $\S \mathrm{I} 8 \mathrm{I}$ ).

I 78 . The cavity in which the posterior end of the trachea and the bronchi lie is the interclavicular air-sac (\$ I64), the ventral wall of which is destroyed by the removal of the sternum.

I79. The sterno-tracheal muscles, paired slender bands, easily mistaken for arteries, passing from the ventral side of the trachea outwards and backwards to the inner surface of the sternum.

I 80. The epigastric or anterior abdominal vein, a small vessel, taking blood from the great omentum, and passing forwards in the falciform ligament towards the anterior border of the liver (see $\$ 253$, p. 240 ).

r $8 \mathrm{r}$, The lungs, dark-red, spongy hodies, seen by turning aside the heart : they are closely applied to the dorsal wall of the anterior part of the cœlome.

I $S_{2}$. The kidneys ( $\left.\$ 2 \mathrm{I}_{4}\right)$ and the reproductive organs ( $\$ 209-2 \mathrm{I} 3$ ), seen by turning aside the intestines in the dorsal region of the posterior part of the cœlome.

$\mathrm{XXV}$. Make a median incision through the skin on the dorsal surface of the head, and reflect it to right or left, so as to expose the skull : beginning a little behind the eyes, cut away the thin cranial roof with a scalpel or pocket-knife, taking great care not to injure the brain, which is in close contact with the bone (consult Fig. 58, p. 255): proceed until the whole of the roof of the skull and the arches of the first two or three vertebræ are removed: when the whole of the dorsal and lateral surfaces of the brain are clear, cut through the spinal cord a short distance behind the brain: lever up the front of the brain with the 
handle of a small scalpel, and cut through, one by one, the nerves which pass from its lower surface: remove the whole organ and place it in strong alcohol or in a saturated solution of zinc chloride for subsequent examination (see $\S \mathrm{LV}$., p. 254).

XXVI. If time permit, continue backwards the incision through the skin, and cut away the arches of all the remaining vertebræ so as to expose the whole spinal cord: in any case, remove the skin from the middle region of the back just between the thighs, and cut away the arches of the middle sacral vertebræ, so as to expose about an inch of the cord in that region : note :

I83. The lumbar enlargement of the cord, and the divergence of its dorsal columns, which enclose a diamondshaped depression, the sinus rhomboidalis, produced by the widening of the central canal of the cord, and covered only by its investing membrane or pia mater.

I84. If the whole cord is exposed, the brachial enlargement will be seen between the shoulders as well as the very great proportional length of the cervical region of the cord.

XVII. Place the subject once more in the supine position (ventral surface uppermost), and make out the following points by merely turning the viscera about, and without cutting or tearing anything :

I85. The relations of the falciform ligament ( $\$ I 70)$ : in front it becomes attached to the pericardium: at its dorsal edge the two layers of which it is composed diverge, forming two laminæ, of which the right passes across the duodenum and becomes attached to the posterior surface of the right lobe of the liver, forming the duodeno-hepatic omentum, while the left becomes attached to the peri- 
toneal investment of the gizzard : both layers are continued backwards as the great omentum ( $(\mathrm{I} 6 \mathrm{I})$.

r86. The characters of the liver: its division into a large right and a small left lobe : its free backwardly directed ventral border: its anterior surface, hollowed out for the reception of the heart : its posterior surface, hollowed by two grooves on the right lobe for the duodenum, and by a deep fossa on the left lobe for the gizzard.

i87. The gastro-hepatic omentum, a sheet of peritoneum connecting the gizzard with the left lobe of the liver.

I88. The mesogaster, a sheet of peritoneum connecting the gizzard with the dorsal body wall.

I 89. The mesentery, a greatly folded sheet of peritoneum supporting the coils of the intestine, and connecting them with the dorsal body wall.

I90. The posterior portion of the intestine, passing along the middle line of the dorsal body wall towards the vent, and suspended by a sheet of peritoneum, the mesorectum : about an inch in front of the vent are two small lateral elevations, the cæca, on the walls of the gut, marking the distinction between the small intestine in front of them, and the large intestine or rectum behind.

I9I. The cloaca, a somewhat dilated chamber, opening externally by the vent, and connected in front with the rectum : its form and relations are best seen by inflating with air from the vent.

XXVIII. Dissect away the great omentum, turn forward the lobes of the liver and dissect out the following structures :

192. The right bile duct, a delicate, whitish tubs, proceeding from the posterior surface of the right lobe 
of the liver, near its dorsal margin, and passing backwards, in the duodeno-hepatic omentum, to open into the distal limb of the duodenum, at about the junction of its middle and distal (anterior) thirds.

I93. The left bile duct, a much wider and shorter tube than the right; also arising from the right lobe of the liver, and passing backwards to open into the proximal limb of the duodenum, about half an inch beyond the pylorus (see $\$ 204,207$ ).

I94. The portal vein, a large vessel, usually gorged with blood, lying in the duodeno-hepatic omentum between the two bile ducts ; it receives blood from the intestines and spleen, and from the right side of the gizzard, and divides into two branches, one of which enters each lobe of the liver.

The portal vein is formed by the union of three chief veins: the gastro-duodenal, receiving blood from the right side of the gizzard, the duodenum, and the last loop of the small intestine (\$§ I73-176); the superior mesenteric, from the main part of the small intestine (jejuntm and ileum, $\S 204$ ); and the inferior mesenteric or coccygeomesenteric ( $(197)$, from the posterior portion of the small intestine, the rectun, and the cloaca.

I95. The three pancreatic ducts : of these one leaves the right side of the anterior end of the pancreas, and passes forwards and to the right, to enter the intestine a short distance beyond the termination of the distal limb of the duodenum; the other two usually spring from about the middle of the gland, also from its right border, and pass slightly forwards, more or less parallel with one another, to enter the duodenum at about the middle of its distal limb.

I96. The left gastric veins, two small vessels contained in the gastro-hepatic omentum, and carrying the 
blood from the left side and anterior edge of the gizzard to the left lobe of the liver.

197. The coccygeo-mesenteric or inferior mesenteric vein (Fig. 54,c.m. $\tau^{\prime}$ ), a large trunk, running in the mesorectum, parallel to the intestine, and receiving veins from the cloaca and rectum: it anastomoses at its dorsal or posterior end with the renal portal veins (see $\$ 2$ I 8 ), and at its anterior end joins the portal vein ( $\$$ r 94 ).

XXIX. Tie a double ligature round the portal vein (the bile ducts may be included), and cut it between the ligatures : if the specimen is injected, the vein may be cut without tying : cut through the gastro-hepatic omentum, and turn the liver over to the right: note:

I98. The proventriculus, a pink, ovoidal body with thick glandular walls, lying immediately in front of the gizzard (see $\S 204$ ), and to the dorsal side of the heart and liver.

I99. 'The spleen, a small, ovoidal, red body, attached to the right side of the proventriculus by a fold of peritoneum, the gastro-splenic omentum.

200. The cœliac artery, seen emerging from between the right lobe of the liver and the proventriculus; its origin from the dorsal aorta is best observed later (\$ 228): it supplies the proventriculus, gizzard, liver, and part of the intestine.

The cœliac divides into two chief trunks: one, the anterior gastric, sends small branches forward to the proventriculus, and then proceeds to the left side of the gizzard ; the other, the gastro-duodenal, supplies the right side of the gizzard, the duodenum, and the last loop of the ileum.

20I. The gastric branch of the vagus, $a$ delicate nerve on the anterior face of the proventriculus, parallel to its artery, and passing backwards to the gizzard. 
202. The splanchnic nerve, running parallel to the gastro-duodenal artery, and supplying the intestine.

203. The anterior (superior) mesenteric artery, about a quarter of an inch posterior to the cœliac, and supplying the greater part of the intestine: to bring it into view the duodenum must be pulled outwards by dissecting away part of its peritoneal investmient.

XXX. Tie a double ligature round the intestine a little anterior to the cæca, including in the ligature the coccygeo-mesenteric vein: cut through the proventriculus as far forwards as possible without injury to the heart, \&c. : remove the alimentary canal between these two points by cutting the mesentery, noting a fibrous membrane (the oblique septum) connected with the dorsal and anterior face of the pericardium in front, and extending across the colome like the roof of a tent: it is attached to the margins of the sternum in front, and to the body walls behind, in such a manner as to separate a sub-puimonary chamber, containing the air-sacs, from the general body cavity. Then observe:

204. The arrangement of the different parts of the alimentary canal : the proventriculus enters the gizzard on its anterior edge : the duodenum leaves it at the anterior end of its right side: following the duodenum is a distinct single loop of small intestine, the jejunum, and after this a coil, consisting of the greater part of the ileum, bound together by a single fold of mesentery: next comes the distal portion of the ileum, consisting of a single loop, of less calibre than the remainder, and usually of a greenish colour : leaving the distal limb of this loop, the ileum widens again, and passes without increase of diameter into the rectum. 
205. The strong, hard walls of the gizzard: the bluish tendons, occupying the central portion of its convex right and left sides, and the muscular fibres radiating from the circumference of each tendon to the edge of the organ.

XI. Unravel the intestine by cutting or tearing through the mesentery, and measure its length as compared with that of the cœlome: then cut open the proventriculus and gizzard, the latter by a vertical incision midway between its two convex faces : also cut open portions of the intestine, and observe :

206. The thick glandular walls of the proventriculus, presenting on their inner surface the well-marked apertures of the gastric glands.

207. The very thick muscular walls of the gizzard, and its lining of thick, horny, yellow or green epithelium: the small stones with which its cavity is largely filled: the place of entrance of the proventriculus, a wide aperture at its anterior end, and the pylorus, or place of exit of the intestine, a crescentic aperture to the right of the former opening.

208. The villi, minute filamentous processes of the mucous membrane of the small intestine : seen to advantage only by examining under water, after well cleaning: in the duodenum and proximal portion of the ileum they are comparatively long and closely set, but in the distal portion of the ileum they become shorter and sparser, and towards the end of that division of the intestine pass into longitudinal ridges.

XXXII. Dissect away the peritoneum and the remains of the posterior air-sacs from the kidneys and reproductive organs, taking care not to injure the postcaval vein ( $\$ 215)$, and, in the 
female, the anterior end of the oviduct ( $\$ 2$ I2) : make out :

In the male.

209. The testes (Fig. 52, ts), two white, ovoidal bodies, about three-quarters of an inch long in the adult, but very

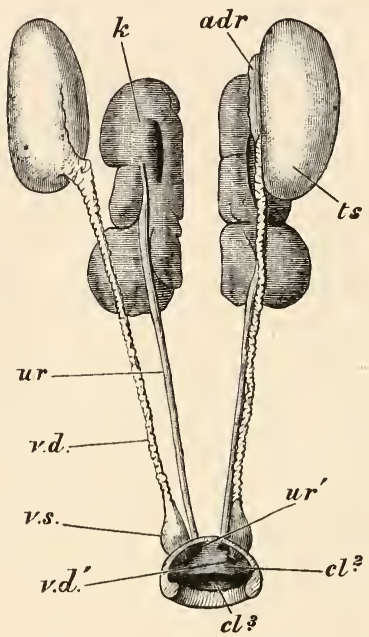

FIG. 52.-Columba livia. The urinogenital organs of an adult male, ventral aspect $\left(\times \frac{2}{3}\right)$.

The right testis and vas deferens are separated from the kidney and ureter, and displaced outwards.

$a d r$, adrenal : $c l .2$, middle, and $c l .3$, posterior, compartment of cloaca : $k$, kidney: $t s$, testis : $u r$, ureter : $u r^{\prime}$, opening of ureter into cloaca : $v . d$, vas deferens : $v . d^{\prime}$, its opening into cloaca $: v . s$, vesicula seminalis.

much smaller in young specimens: they are situated close to one another, at about the level of the anterior border of the thighs.

2 ro. The vas deferens $(v \cdot d)$, a delicate, white tube, convoluted in the adult, but straight in the young, arising from the inner face of each testis, and passing directly 
backwards to the cloaca $(c l)$, where it ends in a dilatation, the vesicula seminalis $(v . s)$ (see $\S 23 \mathrm{I}$, p. 236).

In the female.

2 Ir. The ovary (Fig. 53,ov), a single mass of irregular form, situated at about the same level as the testes ( $\$ 209)$, but somewhat to the left side: it is made up of a number of globular bodies or capsules, each containing an ovarian egg, which, in the adult, vary from the size of a cherry downwards, while in the young hird none may be larger than a pin's head. The single ovary represents that of the left side : a rudiment of the right ovary is sometimes present.

2 I2. The (left) oviduct (l.od), a convoluted tube, with stout walls, about a third of an inch in diameter in the adult, and lying to the left side of the pelvic cavity: posteriorly it enters the left side of the cloaca (see $\$ 232$ ), anteriorly it dilates into a large funnel-shaped termination (l.od"), with delicate membranous walls, and having an antero-posterior diameter of about an inch, which puts the cavity of the oviduct in communication with the cœlome (l.od'"). As some difficulty will probably be experienced in distinguishing between the funnel-like extremity of the oviduct and the peritoneum which supports it, the dissection should be performed under water.

213. The rudiment of the right oviduct $(r . o d)$, a small blind tube, usually about half an inch long, but varying in size in different individuals, attached to the right side of the cloaca.

In both sexes.

$2 \mathrm{I} 4$. The kidneys (Figs. 52 and $53, k$ ), dark red bodies, situated immediately posterior to the lungs, and in close contact with the dorsal body wall : each consists of three well marked lobes - an anterior, a middle, and a posterior- 
which are embedded in cavities furnished by the pelvis and sacrum.

2I5. The postcaval vein (vena cava inferior $s$. posterior,) (Fig. 54, ptc), a large vessel passing from between the anterior ends of the kidneys to the posterior dorsal region of the right lobe of the liver: it enters the substance

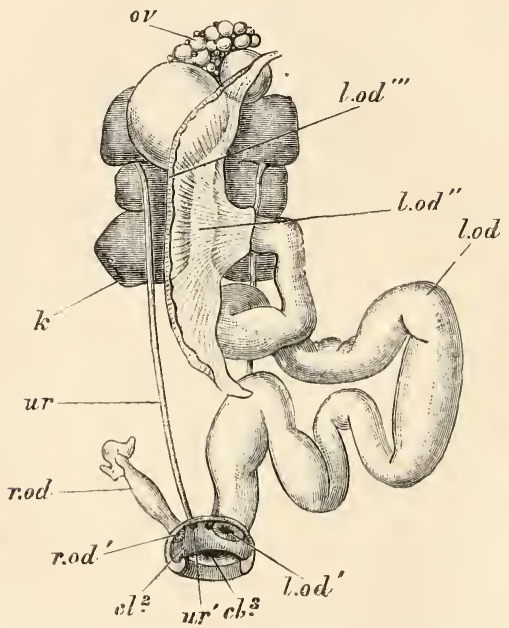

FIG. 53.-Columba livia. The urinogenital organs of an adult female, ventral aspect $\left(\times \frac{2}{3}\right)$.

The peritoneum is removed, but the parts are in situ, except that the left oviduct is partly uncoiled.

cl.2, middle, and $c l .3$, posterior, compartment of cloaca : $k$, kidney ; l.od, left oviduct: $l . o d^{\prime \prime}$, its opening into cloaca: $l . o d^{\prime \prime}$, its funnel-like anterior end : l.od"', its opening into cœlome: $o v$, ovary; $r . o d$, rudimentary right oviduct : $r . o d^{\prime}$, its opening into cloaca : ur, ureter : $u r^{\prime}$, its opening into cloaca.

of that gland, and, emerging from its anterior border, passes forwards to the heart $(\$ 246)$ : it is formed posteriorly by the union of the two iliac veins ( $\$ 218)$, which carry the 
returning blood from the kidneys and reproductive organs, as well as from the hind limbs.

2 I6. The ureter (Figs. 52 and $53, u r$ ), a delicate, membranous tube, arising from the ventral face of each kidney, between its anterior and middle lobes, and passing backwards to the cloaca (see $\S 23 \circ$, p. 235), in the male parallel and internal to the corresponding vas deferens, in the female dorsal to the oviduct. In dissecting out the ureter be careful not to injure the renal (\$220) or the hypogastric (§ 2 I 8$)$ vein.

217 . The adrenals or supra-renal bodies (Fig. $5^{2}$, $a d r$ ), irregular yellowish organs near the anterior end of the kidneys, and closely connected with the iliac reins.

XXXIII. Dissect away, with great care, the testes or the ovary from the kidneys, also dissect out the vasa deferentia or the oviduct, taking care not to injure the renal vein $(\$ 220)$ : turn the reproductive organs and the rectum backwards so as to expose more completely the kidneys and the roof of the pelvic cavity : then dissect out

218 . The renal portal or hypogastric veins (Fig. $54, r . p)$, paired vessels, uniting with one another in the middle line immediately behind the kidneys, and from the point of union sending off a trunk which, uniting with the veins from the cloaca and rectum, forms the coccygeomesenteric vein $\left(c . m . \tau^{\prime}\right)$ : at its anterior end each renal portal vein penetrates the posterior lobe of the kidney. A small median caudal vein $(c)$ bringing blood from the the uropygium pours its blood into the point of union of the renal portals, each of which also receives, just as it enters the kidney, a larger internal iliac vein (i.il) from the roof of the pelvis. 

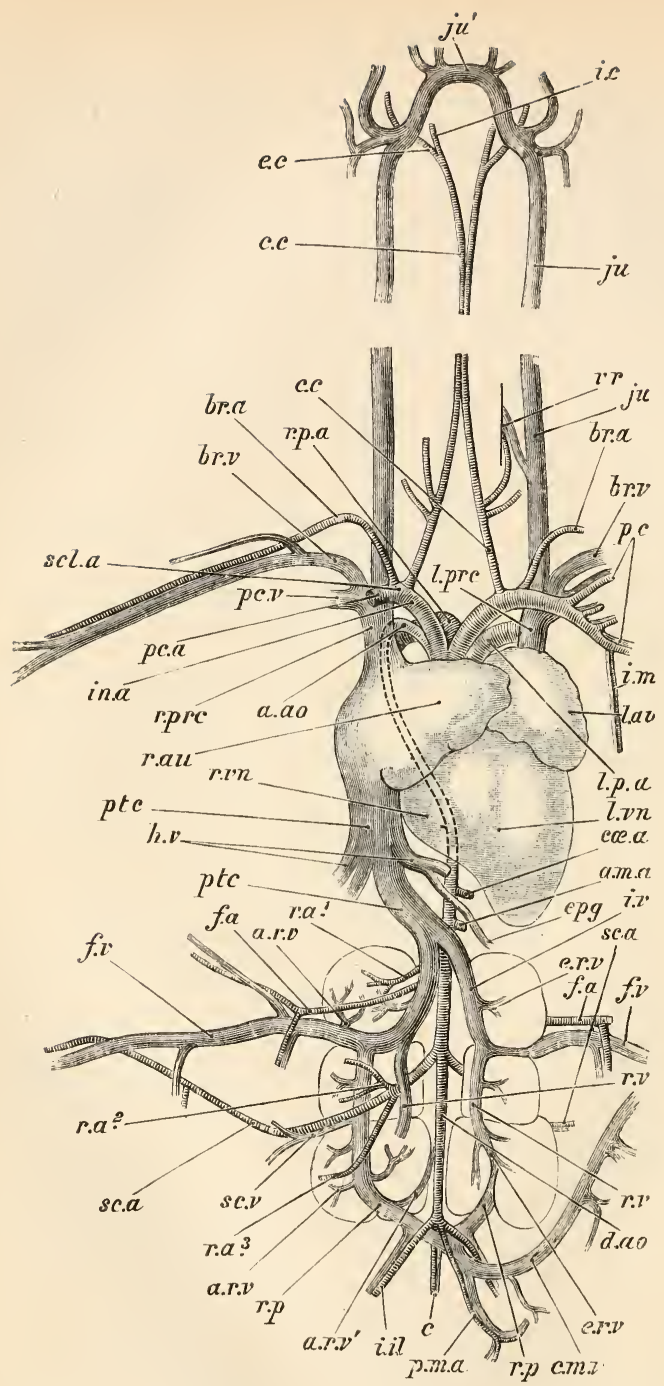

Fig. 54.-Columba livia. The blood vascular system, from the ventral aspect (nat. size). 
The arteries are distinguished by transverse, the veins by longitudinal shading: only the anterior and posterior portions of the vessels of the neck (carotids and jugulars) are shown: the kidneys are represented in outline, the right (left of figure) being supposed to be partly dissected away to show the vessels traversing it: the apex of theart and the coccygeo-mesenteric vein $(c, m . v)$ :are displaced to the left.

$a . a 0$, arch of the aorta: $a . m . a$, anterior mesenteric artery: a.r.v, afferent renal veins : $a \cdot r \cdot v^{\prime}$, vein bringing blood from pelvis into rena! portal system : br.a, brachial artery : br.v, brachial vein : $c$, caudal artery and vein : c.c. common carotid artery : c.m.v, coccygeo-mesenteric vein : $c a . a$, cœliac artery : $d . a o$, dorsal aorta : e.c, external carotid artery: $e p g$, epigattric vein: e.r.v, efferent renal veins: f.a, femoral artery : f.v, fernoral vein $: h . v$, hepatic vein : i.c, internal carotid artery : $i . i l$, internal iliac artery and vein $: i . m$, internal mammary artery and vein : in.a, innominate artery : $i v$, iliac vein : $j u$, jugular vein $: j u$, anastomosis of jugular veins : l.au, left auricle $: l p . a$, left pulmonary artery : l.prc, left precaval vein : l.vn, left ventricle : $p c$, left pectoral arteries and veins $: p c . a$, right pectoral artery : pc.v, right pectoral vein : p.m.a, posterior mesenteric artery : ptc, postcaval vein : r.a. I, anterior, $r . a .2$, middle, and r.a.3, posterior, renal artery : r.an, right auricle : $r . p$, renal portal vein: r.p.a, right pulmonary artery: r.prc, right precaval vein ; $r . v$, renal vein : r. $v^{\prime \prime}$, right ventricle : $s c . a$, sciatic artery : $s c . v$, sciatic vein: scl.a, subclavian artery : $v r$, vertebral artery and vein.

2I9. The femoral vein (f.v), a large trunk returning the greater part of the blood from the leg, ${ }^{1}$ and passing between the anterior and middle lobes of the kidney to join the iliac vein.

Just after entering the body cavity from the leg, the femoral receives a small vein, which runs along the ventral edge of the pubis, and collects the blood from the side wall of the pelvis.

220. The principal renal vein $\left(r . z^{\prime}\right)$, a large longitudinal vessel on the ventral surface of the kidrey, entering the iliac vein at its junction with the femoral.

The renal vein receives several efferent renal veins $(e . r . v)$ from the middle and posterior lobe of the kidney : the blood from the anterior lobe is poured by a separate efferent vein directly into the iliac.

${ }_{1}$ If the muscles are to be dissected in the same specimen, it will be advisable to defer tracing the blood-vessels into the leg for the present, and to follow them at a later stage (see $\$ \$ 32 \$-337$, pp. 252 254), pari passu with the dissection of the muscles of the thigh. 
221. The dorsal aorta (d.ao), a small artery lying in the middle line between the kidneys.

XXXIV. Carefully dissect away one of the kidneys, bit by bit, taking care not to injure the veins in connection with it: make out :

222. The rerral portal vein $(r \cdot p)$, passing through the substance of the kidney to join the femoral just external to the point where the latter unites with the renal vein : during its course through the kidney, the renal portal gives off several small afferent renal veins $(a . r . v)$ to the middle and posterior lobes, the anterior lobe being supplied by similar vessels springing from the anterior face of the femoral vein : the renal portal is joined near the hinder boundary of the kidney by one or two small veins from the sacral region $\left(a . r \cdot v^{\prime}\right)$.

223. The femoral artery $(f . a)$, given off from the aorta between the anterior ends of the kidneys, and passing almost directly outwards to supply the anterior region of the thigh: it leaves the body cavity ventral to the pelvis, and is accompanied by the femoral nerve ( $\$ 293)$.

As the femoral artery leaves the body cavity, it sends off a small vessel which passes along the ventral border of the pubis, accompanying the vein already seen (§ 219$)$, and a branch of the obturator nerve ( $\$ 293$ ).

224. The sciatic artery (sc.a), given off from the aorta at about the junction of the anterior and middle lobes of the kidney; it passes outwards and backwards through the substance of the kidney, emerges from that organ at the junction between its middle and posterior lobes, leaves the body cavity through the ilio-sciatic foramen (§ I04) to reach the thigh, and then passes slightly forwards as well as outwards so as to assume a course parallel to the femoral vein. It is accompanied by the great sciatic nerve $(\$ 294)$.

225. The sciatic vein $(s c . v)$, bringing blood from the posterior part of the leg; it runs parallel to the proximal 
portion of the sciatic artery, and debouches into the renal portal vein.

226. The posterior (or inferior) mesenteric artery $(p . m . a)$, springing from the dorsal aorta at about the level of the posterior border of the kidneys, and going to supply the rectum and cloaca: beyond its origin the aorta becomes the caudal artery $(c)$, which passes backward parallel and ventral to the caudal vein, and from the same point the internal iliac arteries (i.il), are given off, which accompany the corresponding veins ( $\$ 2 \mathrm{I} 8$ ).

227. The renal arteries, of which there are three to each kidney : an anterior $(r . a . \mathrm{I})$, springing from the dorsal aorta just anterior to the origin of the femoral artery, and supplying the anterior lobe; a middle $(r . a .2)$, arising from the sciatic artery near the point where it enters the kidney and passing forwards to the middle lobe ; and a posterior $(r \cdot a \cdot 3)$, arising from the sciatic a little external to the last, and passing backwards to the posterior lobe.

223. The origin of the cœliac $(c a . a)$ and anterior mesenteric arteries (a.m.a) ( $\$ 200,203$ ), from the dorsal aorta.

XXXV. Remove the ventral wall of the cloaca, and note :

229. Its division into three compartments (Figs. 52, 53, and 55), separated from one another by incomplete partitions; the anterior chamber $(c l .1)$ is almost globular when distended, and receives the rectum; the middle chamber $(c l .2)$ is small, separated from the first by an annular fold of the cloacal wall, and receives the urinary and genital ducts $(\$ \S 230-232)$ : the posterior chamber $(\mathrm{cl} .3)$ is separated from the middle by a low annular ridge, contains the aperture of the bursa Fabricii ( $\$ 233$ ), and opens externally by the vent.

230. The small round apertures of the ureters (ur) in the dorsal wall of the middle chamber. 
231. In the male the genital papillæ (Fig. 52,v. $d^{\prime}$ ) conical elevations just external to the urinary apertures : on their apices are the apertures of the vasa deferentia.

232. In the female the aperture of the left oviduct (Fig. 53, l.od'), a considerable opening just external to that of the left ureter: the rudimentary right oviduct has a

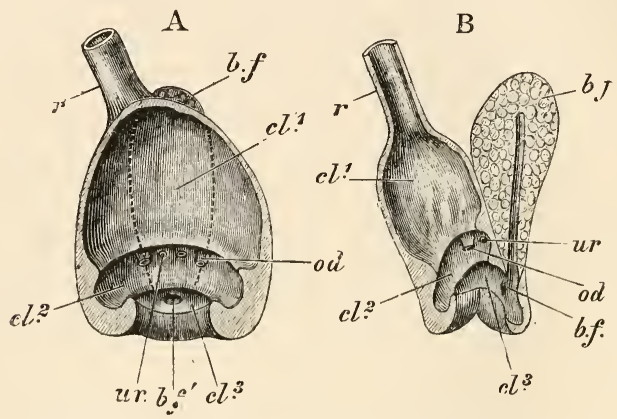

FIG. 55.-Columba livia. The cloaca of a young female. A, opened from the ventral aspect; $B$, in longitudinal section (nat. size).

b. f, bursa Fabricii : $6 . f^{\prime}$, its opening into posterior chamber of cloaca : cl.1, anterior, cl.2, middle, and cl.3, posterior, chamber of cloaca : od, opening of oviducts into middle chamber of cloaca : $r$, rectum : $u r$, opening of ureters into middle chamber of cloaca.

small opening $($ r.oul') similarly situated on the right side: in the young bird the two apertures are of equal size (Fig. 55, od).

233. The aperture of the bursa Fabricii (Fig. 55, b. $f^{\prime}$ ), a small, crescentic opening in the middle of the dorsal wall of the posterior chamber : by drawing the cloaca, backwards, and gradually dissecting it away from its attachments, the bursa is seen as an ovoid, somewhat flattened sac (b.f), with glandular walls, situated between the dorsal wall of the cloaca and the roof of the pelvic cavity: it is best developed in young individuals, when it may attain a length of a quarter to three-quarters of an inch ; in the adult it is usually absent. 
XXXVI. Dissect off the pericardium : carefully clear away the connective tissue and fat from the vessels connected with the base of the heart, and trace them out as far as is necessary to verify the following descriptions : make out :

234. The separation of the heart into an anterior, dark, thin-walled auricular division, and a posterior, lighter coloured, thick-walled ventricular division of a conical shape: the two divisions are separated by a line of fat, which should be carefully removed.

235. The left ventricle (Fig. 54, l.2n), forming the apex of the heart as well as the left convex side of the ventricular division : it is very firm and hard to the touch.

236. The right ventricle $\left(r \cdot \tau^{\prime} n\right)$, much softer to the touch than the left, and forming the right concave side of the ventricular division.

237. 'The right auricle (r.au), forming the right half of the auricular division, and usually gorged with blood after death.

238. The left auricle (l.au), usually in a more collapsed state than the right.

239. The innominate arteries (in.a), two large trunks apparently proceeding from the centre of the base of the heart, and diverging from one another like the limbs of a V : each passes forwards and outwards, and divides into two arteries, the common carotid (c.c), which proceeds almost directly forwards, and the subclavian (scl.a), which continues the direction of the innominate, and almost immediately divides into two : of these the brachial or axillary artery (br.a) passes forwards and outwards, and curving round at the shoulder enters the wing, which it supplies, ${ }^{1}$ while

1 If the muscles are to be dissected in the same specimen, defer tracing the blood-vessels of the wing until its muscles are examined (see $\S 320-327$ ). 
the pectoral artery $(p c . a)$ continues the direction of the innominate and subclavian, which it nearly equals in calibre, and supplies the pectoral muscles.

240. The internal mammary artery (i.m.), a small vessel given off from the posterior surface of the subclavian : it passes backwards along the inner surface of the ribs, and gives off transverse intercostal arteries to the intercostal spaces.

24I. The arch of the aorta (a.ao), situated immediately external to the right innominate artery, of which it has the appearance of being a branch: the aorta is, however, the principal artery arising (see $\$ 274$ ) from the left ventricle, and the innominates are branches given off immediately after its origin, and, owing to the immense size of the pectoral muscles which they supply, exceeding the remainder of the main trunk in calibre: the aorta is seen to pass forwards and slightly outwards or to the right; its further course will be seen at a later stage ( $\$ 257$ ).

242. The pulmonary artery situated just external to the left innominate, and springing from the ventral side of the base of the heart (see $\S 269$ ); almost immediately after its origin it divides into two trunks, the left of which (l.p.a) passes forwards and outwards, parallel to the left innominate, while the other $(r \cdot p \cdot a)$ turns to the right and passes to the dorsal side of the innominates.

243. The right precaval veins (vena cava superior $s$. anterior dextra,) (r.prc), a large vessel situated dorsal and external to the right innominate artery: it is formed by the union of three veins, the jugular $(j u)(\$ 249)$ from the neck, the brachial (br.v) from the wing, and the pectoral $\left(p c v^{\prime}\right)$ from the pectoral muscles: posteriorly it enters the right auricle at its anterior end.

244. The internal mammary vein (i.m), taking a parallel course to the artery of the same name ( $\$ 240)$, and entering the pectoral vein close to its junction with the jugular and subclavian. 
245. The left precaval vein (vena cava superior $s$. anterior sinistra), (l.prc), similarly related to the left innominate artery, and receiving veins symmetrical with those of its fellow: it passes backwards to the dorsal side of the heart, where its further course will be seen subsequently (\$254).

246. The postcaval vein $(p t c)$ has already been seen (§ 215): after emerging from the liver it passes directly forwards and enters the right auricle on the right side of its dorsal wall.

247. The course of the common carotid arteries (c.c), made out by removing the crop and dissecting away the ventral muscles of the neck: each passes forwards and inwards towards the middle line of the neck, and runs parallel and close to its fellow along the ventral aspect of the cervical vertebræ: about an inch behind the head, the arteries diverge again, and each, passing forwards and slightly outwards, divides into an external carotid (e.c), supplying the head generally, and an internal carotid (i.c), supplying the brain.

248. The vertebral artery $\left(\gamma^{\prime} r\right)$, arising from the common carotid in the root of the neck : it passes to the lateral aspect of the neck, and, dipping between the muscles, enters the vertebrarterial canal, and passes forwards to supply the brain and spinal cord.

249. The course of the jugular veins $(j u)$ : they are united with one another by a transverse commissure $\left(j u i^{\prime}\right)$ on the under surface of the skull just behind the velum palati ( $§ 299$ ); - -this is best seen by dissecting away the anterior part of the gullet from the neck muscles and turning it as far forwards as it will go.

The commissural branch receives veins from the palate, and the jugulars themselves from various parts of the head: further back each 
jugular receives veins from the crop, the sides of the neck, and the lymphatic glands ( $\$ 25 \mathrm{I}$ ), and at the level of the posterior end of the latter, the vertebral vein $(v r)$ enters it.

250. The cervical portion of the vagus, a stout nerve accompanying the jugular.

251. The cervical lymphatic glands, paired, reddish, ovoidal bodies, situated one on each side of the root of the neck : each receives an artery from the common carotid, and contributes a vein to the jugular.

XXXVII. Carefully dissect away the liver substance from that part of the postcaval which is embedded in it, and make out:

252. The right and left hepatic veins $(h . v)$, of which the latter is the larger, entering the postcaval just before its exit from the liver.

253. The epigastric vein $(e p g)$ ( $§ 80$ ), entering the left hepatic vein near its junction with the postcaval.

XXXVIII. Ligature the postcaval between the liver and the heart, and cut it through to the distal side of the ligature: turn the apex of the heart forwards and make out :

254. The left precaval (Fig. 56, l.prc) curving round the dorsal side of the left auricle to reach the right auricle.

255. The pulmonary veins $(p . v)$, entering the left auricle in the U-shaped space between the two precavals: sometimes there is a single trunk from each lung, sometimes there are two from each, uniting before they enter the auricle.

256. The course of the right and left pulmonary arteries (r.p.a, l.p.a) ( $(242)$ to the lungs.

257. The arch of the aorta (a.ao) ( $\$ 24 \mathrm{I}$ ), curving over the right bronchus to reach the middle dorsal line, when it becomes the dorsal aorta ( $\$ 22$ I, Fig. 54, d.ao).

XXXIX. Cut through the precavals, the pulmonary arteries and veins, and the aorta, and remove the heart from the body : place it in a dissecting dish 
under water, and note carefully the relations of the great vessels already seen, especially those of the pulmonary veins: then remove the outer walls of both auricles, wash out the contained blood, and make out :

258. The septum auricularum, a thin muscular partition separating the auricles from one another.

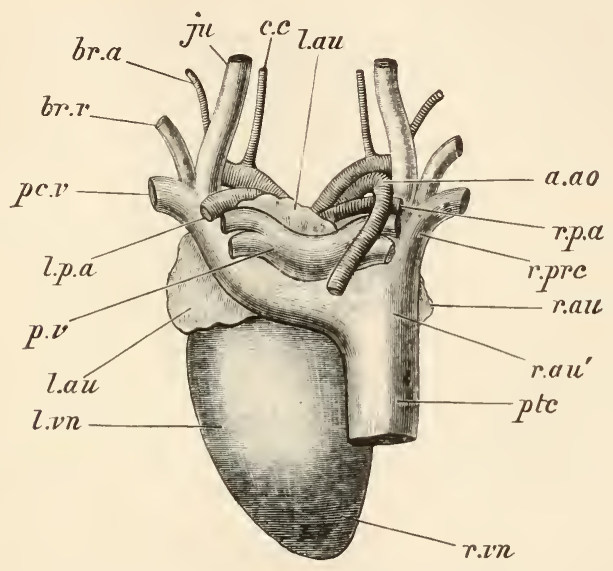

FIG. 56.-Columba livia. The heart, viewed from the dorsal aspect (nat. size).

$a . a o$, arch of aorta: br.a, brachial artery : br.v, brachial vein : c.c, common carotid; $j u$, jugular; l.au, left auricle : l.p.a, left pulmonary artery: l.prc, left precaval: $l . v n$, left ventricle : r.au, right auricle : $r . a u^{\prime}$, its smooth-walled dorsal portion receiving the pre- and postcavals : $r \cdot p . a$, right pulmonary artery: $r \cdot p r c$, right precaval : $r . v n$, right ventricle.

259. The entrance of the right precaval, in the anterior wall of the right auricle.

260. A tunnel-like passage in the dorsal region of the same shamber, leading into the left precaval. 
$26 \mathrm{r}$. The aperture of the postcaval, in the posteroexternal wall of the right auricle, and guarded by a valvular fold of muscle, the Eustachian valve.

262. The fossa ovalis, a thin place in the septum auricularum, marking the position of the embryonic foramen ovale by which the two auricles were placed in communication with one another: it is best seen by holding the septum auricularum up to the light.

263. A deep depression in the dorsal wall of the left auricle, into which the pulmonary veins open.

XL. Cut away both auricles, and all but the origins of the aorta and pulmonary artery, and, looking at the base of the ventricles, note :

264. The rounded left auriculo-ventricular aperture, guarded by the two membranous flaps of the mitral valve. This and the other valves are best seen by filling the ventricles with water and then squeezing them; at every squeeze the auriculo-ventricular valves close, when the pressure is removed they open, and if a sufficient length of the aorta and pulmonary artery is left, the semilunar valves close.

265. The crescentic right auriculo-ventricular aperture, guarded on its outer or convex side by the large, Heshy, right auriculo-ventricular valve ( $\$ 268)$.

266. The rounded apertures of the aorta and pulmonary artery, each guarded by three membranous semilunar valves $(\$ 269)$.

XLI. Open the right ventricle by a V-shaped incision, introducing the scissors into the cut end of the pulmonary artery, cutting obliquely backwards to the apex of the ventricle, and then turning sharply round and passing obliquely forwards, nearly to the anterior border of the ventricle: note: 
267. The convex right face of the septum ventriculorum, or partition between the ventricles, projecting so much into the cavity of the right ventricle as to make it crescentic in section.

268. The right auriculo-ventricular valve, a large flap of muscle, attached partly to the outer edge of the auriculo-ventricular aperture, partly to the outer wall of the ventricle, and depending into the cavity of the latter.

269. The origin of the pulmonary artery from the left side of the anterior end of the ventricle : at the base of the artery the three pocket-shaped pulmonary semilunar valves.

270. The columnæ carneæ, fleshy ridges into which the wall of the ventricle is raised.

XLII. Remove the outer wall of the left ventricle, and note :

27 r. The great thickness of its wall in comparison with that of the right ventricle.

272. The concave left side of the septum ventriculorum.

273. The two membranous flaps of the mitral valve, connected by delicate tendons, the chordæ tendineæ, with small conical elevations of the ventricular wall, the musculi papillares.

274. The aperture of the aorta, guarded by three pocketshaped aortic semilunar valves.

XLIII. Note in the body-cavity, now the heart is removed :

275. The backward continuation of the gullet, dorsal to the trachea, to join the proventriculus.

276. The remains of the intermediate (\$\$ 160, 169) and posterior (\$ I 59) air-sacs, the ventral walls of which will have been removed; they are best seen under water: the anterior-intermediate air-sac covers the greater part of the ventral surface of the lung, the posteriorintermediate lies immediately posterior to the lung: in the anterodorsal region of the posterior-intermediate and posterior sacs are seen 
apertures by which they respectively communicate with the lung; the corresponding aperture of the anterior intermediate is in its anterointernal corner. The apertures of the other air-sacs are much more diffcult to see : that of the sub-bronchial ( $\$ 164)$ lies in its posterior wall just dorsal to the entrance of the bronchus into the lung: the prebronchial extends backwards to the anterior end of the lung and there opens (see $\$ 286$ ).

277. The costo-pulmonary muscles, small fanshaped sheets of muscle, arising from the junctions of the sternal and vertebral ribs, and passing into an aponeurosis which covers the ventral surface of the lungs, between the dorsal walls of the air-sacs and the pleura.

278. The pulmonary pleura, a delicate membrane, continuous with the peritoneum and immediately investing the ventral surface of the lungs: to see it the costopulmonary muscles and their aponeurosis must be removed.

XLIV. Cut through the trachea about an inch anterior to its bifurcation: dissect away the lungs from the dorsal body wall and remove them from the body with the bronchi and posterior end of the trachea: note :

279. The transverse depressions on the dorsal surface of the indistensible lungs, corresponding to the ribs, and the intervening elevations which fit into the intercostal spaces.

280. The dilatation of the posterior end of the trachea into the syrinx (Fig. 57, sy), or "lower larynx."

$28 \mathrm{I}$. The intrinsic syringeal muscles, paired narrow bands arising from the sides of the trachea about an inch in front of its bifurcation, and passing backwards to be inserted into the sides of the syrinx.

282. The complete tracheal rings, their ventral halves bony, their dorsal halves cartilaginous.

283. The bronchial half-rings, along the outer side of each bronchus; the first is bony, the rest are cartilaginous: 
the inner face of each bronchus is flat and membranous, and constitutes the membrana tympaniformis interna.

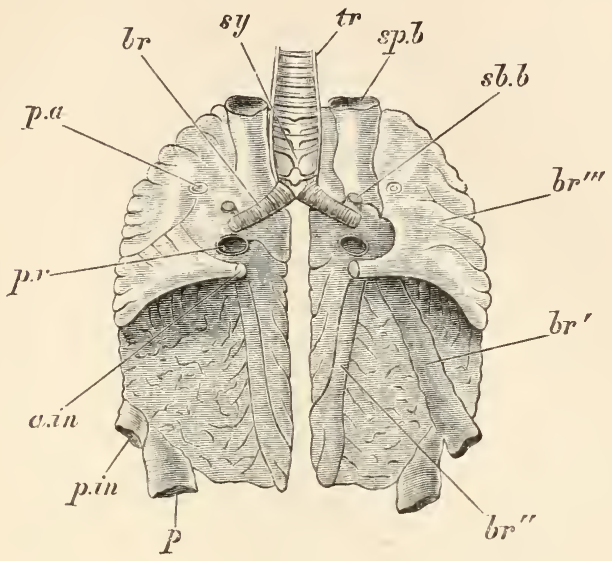

FIG. 57.-Columba livia. The lungs, filled from the trachea with a solid injection and viewed from the ventral aspect (nat. size).

In the left lung the bronchus $\left(b r, b r^{\prime}\right)$ is trared to its termination by dissecting away the overlying substance of the lung.

$h r$, principal bronchus: $b r^{\prime}, b r^{\prime \prime}, b r^{\prime \prime \prime}$, secondary bronchi : $p \cdot a$, pulmonary artery $: p . v$, pulmonary vein $: s y$, syrinx $: t r$, trachea : $s p . b$, aperture for prebronchial air-sac: $s b . b$, for sub-bronchial: $a . i n$, for anterior-intermediate : $p_{.} i n$, for posterior-intermediate $: p$, for posterior (abdominal).

284. The modification of the tracheal rings in the syrinx: the two posterior rings are more widely separated from each other than the rest, and their bony ventral portions send median processes towards one another, which are only separated by a small cartilaginous interval : on the dorsal side these rings are incomplete, but are united with one another on each side of the middle line by a longitudinal cartilaginous bar: the bony first pair of bronchial half-rings are in contact with one anther dorsally and ventrally, and, with the distal tracheal rings just described, inclose a chamber, the tympanum.

285. If the air-sacs, and consequently the lungs, are injected, the secondary bronchi $\left(b r^{\prime}, b r^{\prime \prime}, b r^{\prime \prime \prime}\right)$ will be seen branching out over the anterior and internal regions of the ventral surface of the lungs : they 
have thin membranous walls, and in uninjected specimens are not readily recognised.

286. The apertures in the lung which communicate with the air-sacs, also seen readily only in injected specimens: the aperture of the posterior $\operatorname{sac}(p)$ is at the postero-external angle of the lung: that of the posterior-intermediate $(p . i n)$ just anterior and external to the last : that of the anterior-intermediate $(a . i n)$ at the end of a prominent secondary bronchus on the ventral surface, just posterior to the entrance of the principal bronchus: that of the sub-bronchial $(s b . b)$ is also on the ventral surface, just anterior to the entrance of the chief bronchus : and that of the prebronchial $(s p . b)$ at the anterior end of the lung.

287. By tracing the principal bronchus through the lung (if uninjected, this can be done by passing in a probe and cutting down upon it), it is seen to pass backwards and outwards to the postero-external corner of the lung, and then to divide into two short tubes, the anterior and external of which is connected with the posterior-intermediate air-sac $(\not . i n)$, the posterior with the posterior sac $(a b)$ : the tubes which communicate with the other air-sacs are given off from a dilatation of the principal bronchus, termed the vestibule, just after it enters the ling. Soon after entering the lung the bronchus loses its cartilaginous half-rings.

2S8. Both from the principal bronchus and the secondary bronchi, tertiary bronchi are given off in a pinnate manner: these in their turn give rise to tubes of the fourth order, and so on.

XLV. Sever the bronchi at their entrance into the lungs, and open the syrinx, under water, by the removal of its ventral wall: note :

289. The cushion-like lateral thickenings of the walls of the tympanum, projecting into and narrowing its cavity : the mucous membrane covering them is thicker than elsewhere.

290. The membrana semilunaris, a delicate, inconspicuous, vertical fold of mucous membrane, projecting forwards from the posterior wall of the tympanum at the point of bifurcation of the trachea: it is supported by a delicate bar of cartilage-the pessulus - which extends vertically between the adjacent dorsal and ventral extremities of the first pair of bronchial half-rings. 
XLVI. Dissect away the remains of all the organs still left in the body cavity, and make out on its dorsal wall :

29r. The thoracic spinal nerves, emerging from the intervertebral foramina of the thoracic vertebræ, and passing transversely outwards parallel to and equidistant from the ribs.

292. The brachial plexus, a network of nerves at the root of the neck, formed by the union of the posterior cervical and anterior thoracic spinal nerves: from it are given off the nerves to the shoulder and wings.

293. The lumbar plexus, formed by the nerves which emerge through the intervertebral foramina of the lumbar vertebræ: it gives off the femoral nerve to the front of the thigh, and the small obturator nerve which traverses the obturator fissure ( $(105)$ and goes to the muscles about the acetabulum.

294. The sciatic plexus, formed by nerves emerging from the intervertebral foramina of the sacral vertebræ: it goes mainly to form the great sciatic nerve which accompanies the sciatic artery ( $\$ 224)$.

295. The sympathetic nerves, delicate, paired, longitudinal cords, running close alongside the vertebral column, and having at intervals ganglia, which are connected with the spinal nerves just at their exit from the intervertebral foramina.

Posteriorly the two sympathetics unite in a single small ganglion impar : anteriorly each forms a large posterior cervical ganglion lying ventral to the brachial plexus, and thence passes along the vertebrarterial canal, alongside the vertebral artery, to the head.

XLVII. Dissect away the skin from the dorsal surface of the uropygium, and note:

296. The uropygial gland, a whitish, bilobed organ, 
about a quarter of an inch long, with its backwardly directed apex passing into a duct which opens in a distinct median papilla.

XLVIII. Cut through one side of the lower jaw near its articulation, open the mouth widely, and observe :

297. The palate, or roof of the mouth, marked by a median longitudinal slit, bounded by two fimbriated folds of mucous membrane, between which are the posterior nares.

298. At the posterior end of the palatal folds is the single median aperture of the Eustachian tubes, which communicates, right and left, with the tympanic cavities (\$ 3 ז 8 ).

299. The velum palati, a bilobed flap of mucous membrane, with fimbriated edges, forming the posterior termination of the palate.

300. The tongue, pointed in front, bifid and fimbriated behind.

30I. The glottis, an oval aperture just behind the root of the tongue, leading into the trachea: its margins are slightly fimbriated, and immediately behind it is a bilobed fimbriated fold of mucous membrane.

302. The wide pharynx, leading into the gullet.

303. By dissecting away the mucous membrane of the glottis, the larynx is found, a cartilaginous apparatus forming the anterior end of the trachea.

The larynx consists of the following parts:-(a) the thyroid, a partly ossified cartilaginous plate, bent upon itself in the form of a dorsally incomplete ring, wide and scoop-shaped ventrally, narrow dorsally: $(b)$ the cricoid, a small longitudinal rod of cartilage, interposed between the dorsal ends of the thyroid: $(c)$ the arytenoids, paired, somewhat triangular cartilages, ossified at their proximal ends, where they articulate with facets on the anterior end of the cricoid; 
they form the actual supports of the glottis. The two anterior rings of the trachea are incomplete dorsally, and the first has its dursal extremities fused with the thyroid.

XLIX. Cut away the outer wall of one of the nasal cavities, first passing a probe from the anterior to the posterior nares: note:

304. The elevation caused by the turbinal ( $\$ 72$ ), covered by the delicate Schneiderian membrane.

305. The relations of the anterior and posterior nares to the nasal cavity.

L. Remove the dorsal wall of the orbit: observe the four straight and two oblique muscles, which have the same relations as in the lower vertebrata (see pp. 77,78 , s $19 \mathrm{I}-203$ ) : remove the eye with the nictitating membrane by cutting through its muscles and the optic nerve : observe :

306. The lacrymal gland, a whitish irregular body in the postero-dorsal region of the orbit.

307. The Harderian gland, a small pinkish body in the anterior region of the orbit.

308. The form of the eye : its inner half has the usual hemispherical figure, but its outer half is somewhat obtusely conical.

309. The entrance of the optic nerve, in the centre of the inner hemisphere.

310. The bursalis, a somewhat quadrate shoot of muscle, arising from the dorsal region of the inner hemisphere by a convex edge, and passing downwards to end in a concave edge just over the optic nerve: the lower edge forms a tubular tendinous sheath.

3II. The pyramidalis, a triangular muscle, arising from the antero-ventral region of the inner hemisphere; it passes upwards and backwards, and, just in front of the 
optic nerve, ends in a tendon which passes through the sheath of the bursalis, then proceeds downwards and backwards, curves round the postero-ventral margin of the eyeball, and finally passes forwards to be inserted into the edge of the nictitating membrane.

312. The sclerotic, cornea, iris, and pupil have the same relations as in other Vertebrata (see p. 84, §§ 224, 225 ).

3r3. The sclerotic plates, small flat bones, arranged in an imbricating manner round the sclerotic just external to its junction with the cornea.

I.I. Divide the eye, by an equatorial incision, into inner and outer hemispheres : note :

314. The pecten, a black plaited structure, in the antero-ventral region of the inner hemisphere, having a curved free edge, and an attached edge which extends from the entrance of the optic nerve downwards and forwards, parallel to the course of the pyramidalis ( $\$ 3 \mathrm{II}$ ): it consists of a fold of the choroid, projecting through a fissure in the retina. It should be removed and examined under a low power of the microscope.

3I5. The choroid, retina, lens, and aqueous and vitreous humours have the same relations as in the other Vertebrata (see pp. 84, 85, §§ 224-228).

3I6. In the outer hemisphere, the choroid processes, radiating folds, developed in the outer part of the choroid, between the outer edge of the iris and the ora serrata or free border of the retina.

LII. Cut away the walls of the external auditory meatus ( $\$ 128$ ), and make out:

317. The tympanic membrane, a thin sheet of fibrous tissue stretched across the bottom of the external meatus.

318. By carefully removing the tympanic membrane, the 
tympanic cavity is reached, a small chamber, separated from the external meatus by the tympanic membrane, and communicating with the cavity of the mouth by the Eustachian tube ( $(298)$, into which a bristle should be passed. 319. The relations of the columella to the tympanic cavity (see $\S 6 \mathrm{I}, \mathrm{p} .195$ ).

LIII. Carefully remove the skin from the shoulder and wing, and dissect off the fascia, a strong sheath of connective tissue which covers the muscles, until the following can be made out:

320. The tensor patagii longus, a small conical slip of muscle, arising from the antero-external region of the pectoralis major: it receives fibres from the tensor patagii brevis ( $\$ 25)$, and passes distally into a long elastic tendon, lying in the border of the alar membrane ( $\$$ r 35 ), and ir.serted into the skin on the pre-axial border of the manus.

321. The biceps, a large muscle forming the fleshy mass in the anterior border of the upper arm, and acting as the chief flexor of the fore-arm.

322. The triceps, forming the fleshy mass on the posterior aspect of the upper arm, and acting as extensor of the fore-arm.

323. The tensor patagii accessorius, a small squarish mass of muscle lying in the proximal region of the alar membrane: it arises from the fascia of the biceps; its anterior border is connected by fascia with the tendon of the tensor longus, and from its posterior border a long stout tendon is given off which passes outwards, soon becoming parallel to the tendon of the tensor longus, and having a common insertion with it.

324. The extensor metacarpi radialis longus, a muscle forming the anterior or preaxial edge of the forearm, and acting as extensor of the manus. 
325. The tensor patagii brevis, a oroad sheet of muscle covering the shoulder on its dorsal aspect; it arises from the dorsal ends of the coracoid and furcula, passes backwards, parallel to the humerus and partly covering the triceps, contributes fibres to the tensor longus, and is inserted by a broad tendon into the fascia of the extensor metacarpi radialis longus.

326. The insertion of the subclavius ( $\$$ I66): the muscle passes anteriorly into a tendon which proceeds forwards and outwards to the foramen triosseum: by cutting through and reflecting the tensor brevis, the tendon is seen to emerge from the foramen on its dorsal side and to be inserted into the dorsal surface of the humerus, near the greater tuberosity $(\$ 95)$ : by pulling upon the muscle, the wing is raised; the subclavius is the chief elevator of the upper arm.

327. The insertion of the pectoralis major (\$ I 53), by cutting through and reflecting the biceps: its fibres are gathered into a broad flat tendon which is attached along the whole extent of the greater tuberosity: the pectoralis major is the principal depressor of the wing.

LIV. Remove the skin from the leg, and, by carefully dissecting away the fascia, clear the muscles and their tendons sufficiently to show :

328. The extensor tendons of the digits, on the anterior (dorsal) side of the tarso-metatarsus : those of the second, third, and fourth, or forwardly-directed digits, are formed by the trifurcation of a single tendon, which, traced into the shank, is found to be the tendon of insertion of a small muscle, the extensor longus digitorum, which arises from the anterior face of the proximal end of the tibia : the extensor tendon of the first digit, or hallux, springs from a small distinct muscle, the extensor hallucis brevis, 
which arises from the proximal end of the tarso-metatarsus. Distally the extensor tendons run along the dorsal sides of the digits, closely bound down by fascia, and are inserted into the bases of the distal phalanges.

329. The gastrocnemius, a large muscle consisting of distinct internal and external portions, and forming the outer layer of the fleshy mass on the posterior surface of the shank : its two heads arise respectively from the inner and outer surfaces of the proximal end of the tibia, the internal head arising also from the distal end of the femur: the two heads unite in a broad, flat tendon, which is inserted into the proximal end of the posterior surface of the tarso-metatarsus : its action is to straighten the foot on the shank.

330. The flexor tendons of the digits, a large number of readily separable tendons on the posterior (ventral) surface of the tarso-metatarsus: traced into the leg they are found to be the distal tendons of a number of separate muscles, the flexores digitorum, which arise from the posterior and lateral surfaces of the tibia; to see them the gastrocnemius should be cut through and reflected. Distally, the flexor tendons can be traced into the toes, either two or three to each (see $\$ \S 33 \mathrm{I}-334$ ).

33I. In the fourth or outermost toe there are two flexor tendons, one being superficial or ventral to the other : the superficial or perforated tendon (springing from the flexor perforatus muscle) sends off lateral slips to the proximal phalanx, and then forks, one division going to each side of the second and third phalanges: the deep or perforating tendon, lying between the former and the bone, passes between the division of the perforatus, along the ventral surface of the phalanges, and is inserted into the proximal end of the distal phalanx.

332. In the third digit the arrangement is complicated by the presence of two perforated tendons : of these the bifurcations of one are inserted into the proximal, those of the other into the second, phalanx : so that through the fork of the first or superficial perforated tendon both the second perforated and the perforating tendons pass. 
333. In the second digit the arrangement is similar, except that the first or superficial perforated tendon, instead of forking, forms a sheath for the other two tendons at the proximal end of the first phalanx.

334. In the first digit or hallux, there are only two flexor tendons; of these the perforating tendon is superficial, and the perforated forms a sheath for it at the proximal end of the first phalanx.

335. The perforating tendons of the second, third, and fourth digits are all formed by the trifurcations of a single tendon, lying eeper than the perforated tendons, and belonging to a large muscle, the peroneus medius : this tendon is joined by a slip from that of the flexor perforans, which is continued to form the perforating tendon of the hallux.

336. The ambiens, a small, slender muscle, lying superficially on the inner surface of the thigh, just over and parallel to the femur : it arises from the pubis immediately ventral to the acetabulum : distally it passes into a long, slender tendon, which, on reaching the knee, penetrates the fibrous capsule of the joint, curves round to the outer side, passing at the same time distalwards, and finally joins the flexor perforatus of the second and third digits.

337. If, on the undissected side, where the tendons are not displaced, the shank is flexed on the thigh and the tarso-metatarsus on the shank, as in perching, the toes will undergo complete flexion: this is chiefly due to the fact that in the flexion of the tarso-metatarsus on the shank the flexor tendons of the toes are put upon the stretch as they pass the mesotarsal articulation ( $\S$ II 7 ): it is also aided by the flexion of the shank upon the thigh producing tension of the ambiens tendon, which, in its turn, acts upon the flexors of the digits.

LV. In the hardened brain notice the following external characters :

338. The medulla oblongata (Fig. 58, m.o), continuous behind with the spinal cord, and like it composed externally of white matter : ${ }^{1}$ it greatly exceeds the cord in

1 The distinction between white and grey matter is not obvious in the hardened brain; it is therefore convenient to have a freshly removed brain for comparison. 
diameter, and at the junction of the two there is a strong ventral flexure: it is marked ventrally with a median longitudinal groove, separating the ventral pyramids: on the upper side are the dorsal pyramids, which, passing from behind forwards, diverge, leaving a median dorsal hollow, the fourth ventricle (Fig. 59, v.4).

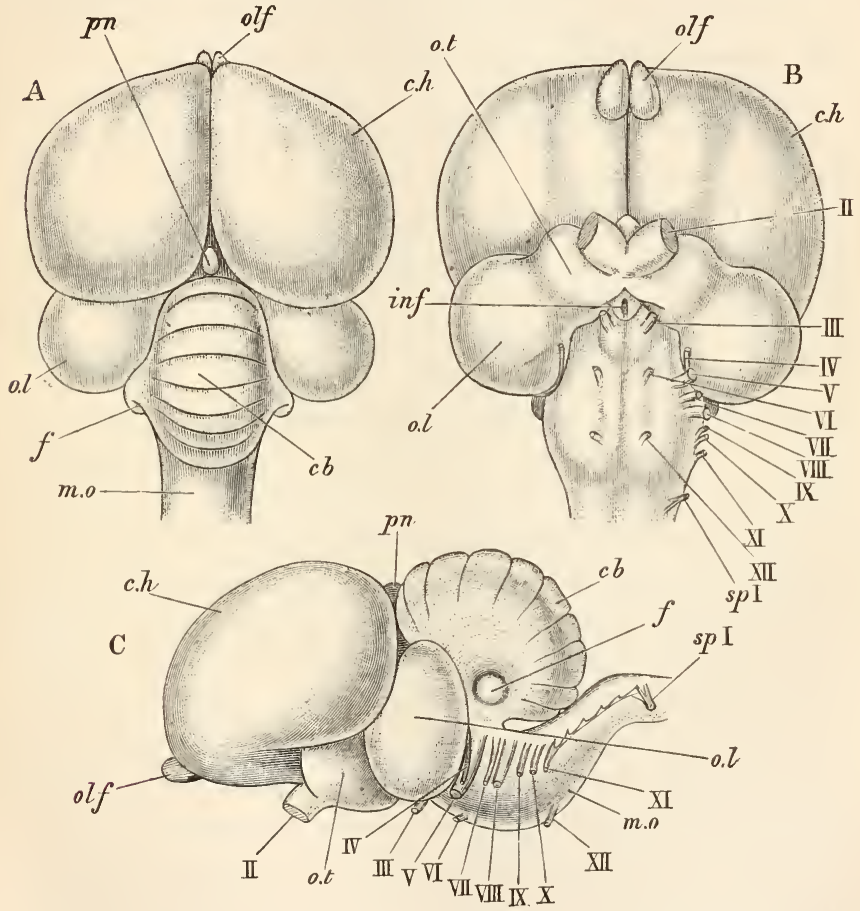

FIG. 58.-Columba livia. The brain. A, from above; B, from beneath; $\mathrm{C}$, from the left side $(\times 2)$.

$c b$, cerebellum: $c . h$, cerebral hemispheres : $f$, flucculus : inf, infundibulum : $m .0$, medulla oblongata : $0 . l$, optic lobes : olf, olfactory lobes : $0 . t$, optic tracts : pn, pineal body : II-XII, roots of cerebral nerves: $s p . I$, root of first spinal nerve. 
339. The cerebellum (Fig. $5^{8}, c b$ ), a median, laterally compressed, crest-like mass, lying above the anterior part of the medulla oblongata; it is marked by transverse furrows or sulci, between which are elevations or gyri : on the lateral surfaces of the cerebellum the sulci and gyri radiate from an area on which is a slight elevation, the flocculus $(f)$ : the cerebellum has a pink tint in the fresh brain, being composed externally of grey matter.

340. The optic lobes (o.l), paired, white, ovoidal bodies, situated one on either side, immediately in front of the medulla and below the cerebellum.

34 I. The cerebral hemispheres $(c . h)$, paired, obtusely conical bodies, in close contact with one another, and situated immediately in front of the cerebellum and optic lobes, with both of which their hinder surfaces are in contact: they consist externally of grey matter.

342. The pineal body $(p n)$, a small rounded mass on the dorsal surface of the brain, wedged in between the cerebral hemispheres and the cerebellum.

343. The olfactory lobes (olf), small blunt projections, connected one with the anterior end or apex of each cerebral hemisphere : from them the olfactory nerves (first pair of cerebral nerves) are given off to the nasal sacs.

344. The optic tracts (o.t), forming a broad, white transverse band, concave forwards, between the optic lobes, on the lower surface of the brain: from their anterior median region the optic (second) nerves (II) spring.

345. The infundibulum (inf), a rounded area with a central aperture, just posterior to the optic tracts: to it is attached the pituitary body, which is usually detached in removing the brain from the skull.

346. The third nerve (III), arising close behind the infundibulum, a short distance from the middle line, from a 
part of the brain not separated off from the medulla oblongata, but answering to the crura cerebri.

347. The fourth nerve (IV), arising from the dorsal side of the medulla oblongata at its anterior end : it emerges between the medulla and the cerebellum, and passes downwards in the groove between the medulla and the optic lobe to reach the ventral surface of the brain.

348. The fifth nerve (v), a large trunk arising from the lateral surface of the medulla oblongata just behind the optic lobe: its fibres extend upwards quite to the dorsal edge of the medulla.

The fifth nerve arises by two roots, the larger of which presents an enlargement, the Gasserian ganglion.

349. The sixth nerve (VI), arising from the ventral surface of the medulla near the middle line and just internal to the point of origin of the fifth.

350. The seventh nerve (viI), a small trunk arising close behind the fifth.

35 . The eighth or auditory nerve (VIII), a large, soft trunk arising close behind the seventh : its fibres are traceable upwards to the dorsal pyramids of the medulla.

352. The ninth (Ix), tenth (x), and eleventh (XI) nerves, arising together close behind the eighth: they have small roots, the tenth (vagus) being the largest, and the eleventh (spinal accessory) distinguished by being traceable upwards and backwards alongside the medulla oblongata to the spinal cord, from which it arises between the roots of the spinal nerves, also receiving. fibres from the medulia.

353. The twelfth nerve or hypoglossal (XII), a small trunk arising from the ventral surface of the medulla internal to the eleventh. 
LVI. Separate the hemispheres from one another by gently pressing them outwards, noting the absence of any connection between them: make a small incision in the inner wall of one of them near its postero-dorsal corner, so as to lay bare its cavity ( $\$ 357$ ): carefully cut away enough of the inner and posterior walls of the hemisphere to expose the whole of this cavity: also remove the cerebellum by cutting through its lateral attachments to the medulla oblongata ( $\S 354)$ : note:

354. The attachment of the cerebellum to the medulla by strong, paired pillars of white matter, the peduncles (Fig. $59, A, p)$ : from its attachments the cerebellum extends backwards over the medulla and forwards between the optic lobes.

355. The optic commissure (o.c), a flat, horizontal band of white fibres uniting the optic lobes beneath the anterior prolongation of the cerebellum.

356. The thalamencephalon (thc), lying immediately in front of the optic commissure, and hidden in the entire brain by the meeting of the cerebral hemispheres with the cerebellum: it consists of small paired masses of grey matter, the optic thalami, which, if the dissection has been carefully conducted, will be seen to be covered by a thin sheet of pia matter full of blood-vessels, the velum interpositum : this is best seen in a fresh brain.

357. The lateral ventricle, or cavity of the cerebral hemisphere, an irregular chamber with very thin inner, upper, and posterior walls, but having its floor thickened and its dimensions greatly diminished by a large mass of white matter, the corpus striatum (c.s).

$35^{8}$. The third ventricle $(v \cdot 3)$, a vertical cleft between the optic thalami, roofed over by the velum interpositum, 
and bounded in part by a thin plate of grey matter, the lamina terminalis, (l.t).

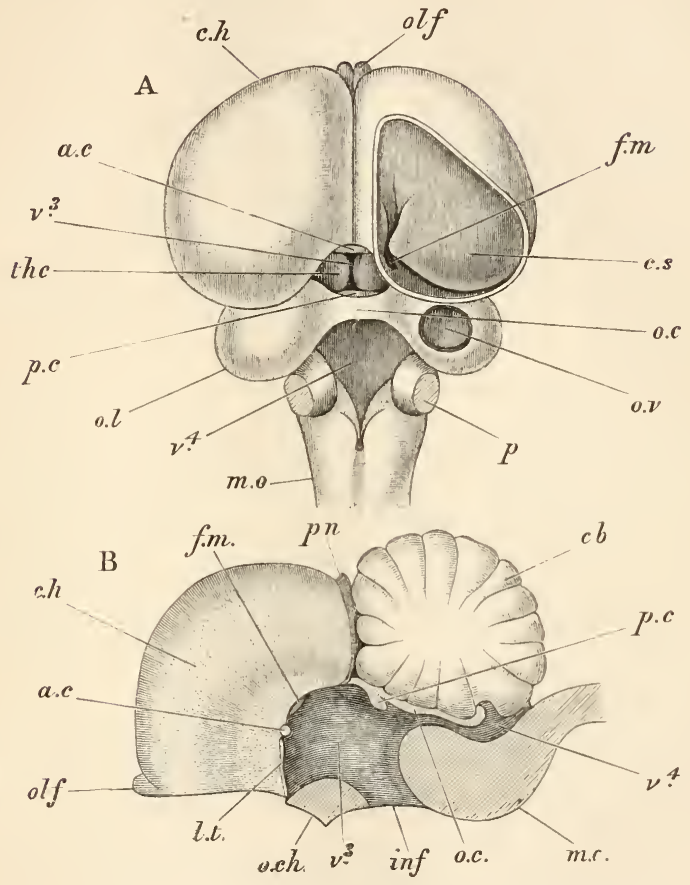

FIG. 59. - Columba livia. The brain. A, dissected from the dorsal aspect; $B$, in longitudinal vertical section $(\times 2)$.

In $\mathrm{A}$, the hemispheres are somewhat displaced outwards, and the roofs of the right lateral and optic ventricles are removed, as well as the cerebellum and the velum interpositum.

a.c, anterior commissure : $c b$, cerebellum : $c . h$, cerebral hemispheres : c.s, corpus striatum : f.m, foramen of Monro: inf, infundibulum : $m . o$, medulla oblongata: o.c, optic commissure: o.ch, optic chiasma: o.l, optic lobes : olf, olfactory lobes : $0 . v$, optic ventricle : $p$. peduncles of cerebellum : $p . c$, posterior commissure : $p n$, pineal body: the, thalamencephalon : $v .3$, third ventricle : $v .4$, fourth ventricle. 
359. The foramen of Monro $(f . m)$, a small aperture in the inner wall of the lateral ventricle at the level of the anterior end of the thalamencephalon : it communicates with the third ventricle and transmits a vascular fold of pia mater, the choroid plexus, which is continuous with the velum interpositum.

360. The anterior commissure (a.c), a white, transverse cord connecting the corpora striata with one another, and lying just below the foramina of Monro.

$36 \mathrm{r}$. The posterior commissure $(p . c)$, a similar cord uniting the thalami immediately in front of the optic commissure.

362. The fourth ventricle (v.4), a wide flat cavity on the dorsal surface of the medulla oblongata, covered, in the entire brain, partly by the cerebellum, partly by pia mater.

LVII. Carefully remove the optic commissure and the dorsal wall of one or both optic lobes: observe :

363. The aqueduct of Sylvius (Fig. 59, B), a median passage placing the third and fourth ventricles in communication with one another.

364. The optic ventricle (Fig. 59, A, 0.v), a cavity in each optic lobe, communicating internally with the aqueduct of Sylvius, and with it representing the simple mesocole of the lower Vertebrata (see $\S$ I 8I, p. 73, and $\S 86$, p. 23).

LVIII. Make a longitudinal vertical section of another hardened brain, and note :-

365. The continuity of the third ventricle, aqueduct of Sylvius, and fourth ventricle (Fig. 59, B, v*3,v.4).

366. The relation of the foramen of Monro $(f . m)$ to the third ventricle, and of the apertures of the optic ventricle to the aqueduct of Sylvius.

367. The downward continuation of the third ventricle into the infundibulum (inf). 
368. The relations of the anterior (a.c), posterior ( $p . c)$, and optic (o.c) commissures.

369. The arbor vitæ, or foliated appearance produced in the section of the cerebellum by the superficial grey matter being continued inwards into the sulci, and investing the outward prolongation of the central white matter into the gyri. If a second brain is not at hand, this may be seen in a section of the cerebellum removed in $\S$ LVI : it is best seen, however, in a fresh brain. 
T HE R A B B I T (Lepus cuniculus).

\section{A.-THE SKELETON.}

I. It is advisable to have two skeletons for examination, one of an adult animal, the other of a specimen about six weeks old; the latter is the more important of the two. In the adult skeleton the bones are best kept in their natural connection, in the young specimen they should be all separated from one another by prolonged maceration, or by boiling for a short time in water. Of the entire skull a longitudinal vertical section should be made with a fine saw.

II. In the skeleton as a whole, make out its division into

I. The axial skeleton, consisting of the skull ( $(4)$ and vertebral column, with the ribs and sternum.

2. The appendicular skeleton, consisting of the shoulder-girdle $(\S 5)$, the hip-girdle or pelvis $(\S 6)$, and the fore- and hind-limbs.

3. The division of the vertebral column into regions: the cervical region, containing the seven ribless vertebræ which immediately follow the head; the thoracic region, 
consisting of twelve or sometimes thirteen rib-bearing vertebræ; the lumbar region, containing seven or sometimes six large vertebræ without ribs; the sacral region, consisting of four vertebræ more or less completely fused into a single bone, the sacrum; and lastly, the caudal region, containing about fifteen vertebræ, which extend from the sacrum to the end of the tail.

4. The skull, consisting of the skull proper (brain-case and upper jaw), the lower jaw or mandible, and the tongue-bone or hyoid.

5. The shoulder-girdle, consisting on each side of the scapula, or blade-bone, and of the small clavicle, or collar-bone.

6. The hip-girdle, consisting of large paired bones, the ossa innominata, united both with one another and with the sacrum.

7. The fore-limb, divisibie into three parts: a proximal division, consisting of a single bone, the humerus; a middle division, containing two bones, an inner, the radius, and an outer, the ulna ; and a distal division, the manus, made up of the carpus or wrist, and of five digits.

8. The hind-limb, also divisible into three parts: a proximal, containing a single bone, the femur; a middle, consisting of a large inner bone, the tibia, and a small outer one, the fibula ; and a distal, the pes, consisting of the tarsus or ankle, and of four digits.

III. In one of the first half-dozen thoracic vertebræ note :

9. The centrum or body, a sub-trihedral mass of bone forming the ventral portion of the vertebra.

Iо. The neurapophyses or neural processes, paired bony rods, passing upwards and slightly outwards, one from each side of the centrum and uniting with one another 
above to form the neural arch, through which the spinal cord passes: each neurapophysis consists of a ventral, somewhat cylindrical portion, the peduncle, and of a dorsal flattened portion, the lamina.

I I. The neural spine, an elongated, backwardly directed process proceeding from the apex of the neural arch at the junction of its two laminæ.

I2. The transverse processes, short stout rods proceeding directly outwards one from each side of the neural arch at the junction of the peduncle and lamina.

I3. The anterior zygapophysis, a small horizontal process projecting forwards from the anterior face of each neurapophysis at the junction of the peduncle and lamina: its dorsal surface is flat and smooth, forming an articular facet.

14. The posterior zygapophysis, a slight projection of the postero-ventral region of each lamina, bearing an articular facet on its under side. ${ }^{\mathrm{I}}$

I5. In the young skeleton, the epiphyses, two thin bony disks, closely applied one to the anterior the other to the posterior face of the centrum to which they are ankylosed in the adult.

In the young subject the epiphyses easily separate from the vertebra proper, to which, indeed, they are less firmly attached than to the intervertebral ligaments $(\$ 27)$; it therefore often happens, in maceration, that an epiphysis becomes detached from its own vertebra, but remains united to the next by the intervertebral ligament.

The free surfaces of the epiphyses are smooth; their inner surfaces, in contact with the centrum, are rough, as also are the faces of the centrum itself : the centrum presents two oblique grooves, corresponding to the neuro-central sutures (\$ I 6 ), and equally well marked whether the latter

1 It is useful to remember that the anterior face of a vertebra can always be told by observing the zygapophyses : the facets of the anterior zygapophyses look upwards, inwards, or forwards, those of the posterior zygapophyses downwards, outwards, or backwards. 
have disappeared or not; the inner face of the epiphysis bears two ridges corresponding with these grooves. It is thus easy to tell from the appearance of a vertebra whether its epiphyses are present or not.

I6. The neuro-central sutures, oblique division planes separating the centrum proper, in the young animal, into three portions; the middle piece is a distinct bone, the lateral pieces are continuous with the neurapophyses.

I 7. The tubercular facet, a small surface near the end of the transverse process and serving for the articulation of the tubercle of a rib ( $\$ 29)$.

I8. The capitular demi-facets, two semilunar surfaces at the junction of the centrum and neurapophysis, one at the anterior the other at the posterior border of the vertebra, and furnished partly by the vertebra proper, partly by the epiphysis : the anterior demi-facet serves for the articulation of the head of the rib ( $\$ 29$ ) belonging to the vertebra in question, the posterior for that of the next following rib.

IV. In the remainder of the vertebral column note :

I9. The last three thoracic vertebræ: note the diminished size of the neural spine, which is vertical in the eleventh (anticlinal vertebra), and slopes slightly forwards in the twelfth, becoming at the same time compressed from side to side; the shortmess of the transverse processes, and the absence of a tubercular facet; the entire capitular facet for the head of the rib, there being no lialf-facet on the posterior edge of the centrum; the metapophysis, a large process near the anterior border of the neural arch, just posterior to the anterior zygapophysis, and projecting upwards and slightly forwards.

20. The lumbar vertebræ: note the short, forwardly directed, laterally compressed neural spine; the large metapophyses; the anterior zygapophyses looking directly inwards, the posterior outwards; the large transverse 
processes increasing in size from the first lumbar vertebra to the last, and turning forwards; the hypapophysis, a short flattened process from the under surface of the centrum of the second and third; and the anapophysis, a small pointed process, projecting backwards from the posterior edge of the neural arch beneath the posterior zygapophysis.

2 I. The sacral vertebræ: these are much like the lumbar, but have smaller metapophyses, and no anapophyses or hypapophyses. In the first the spine is usualiy directed forwards, in the second it is vertical, and in the third and fourth directed backwards. The first and second only give attachment to the hip-girdle, for which purpose they are provided with great expanded lateral plates with roughened edges, which answer to sacral ribs.

22. The caudal vertebræ, at first like the sacral, but gradually diminishing in size and complexity until they are reduced to mere centra.

23. The last (seventh) cervical vertebra : it somewhat resembles the anterior thoracic, but the transverse process has no facet, and the centrum a demi-facet only on its hinder edge. The centrum also is so altered in shape that its anterior face looks downwards as well as forwards, and its posterior face upwards as well as backwards.

24. The third to the sixth cervical vertebræ: these have the centrum with its anterior face looking downwards and forwards, and its posterior face upwards and backwards; the transverse processes are divided into two parts, one of which projects directly outwards, while the other (inferior lamina) forms a flat irregular plate, attached along the whole inferolateral edge of the centrum. An aperture, the vertebrarterial canal, perforates the base of the transverse process, and, during life, transmits the vertebral artery ( $\$ 25^{\circ}$ ). 
25. The second cervical vertebra, or axis; the centrum is produced anteriorly into a strong, bluntly-pointed peg, the odontoid process; on the under surface of this is an articular surface, and on each side of its base is another large surface; all these are for articulation with the first vertebra; the spine is flattened from side to side, and greatly produced from before backwards; the transverse processes are small, directed backwards, and perforated by the vertebrarterial canal. In young rabbits (six weeks) this axis consists of three pieces, one forming the arch, another the posterior part of the centrum, and the third the anterior part of the centrum with the odontoid process : the latter ossification (os odontoideum) answers to part of the centrum of the atlas.

26. The first cervical vertebra or atlas: the centrum is reduced to a mere flat plate, hardly thicker than the arch, and with less than half the antero-posterior dimensions of the latter; the transverse processes are large, flattened from above downwards, and perforated; just within and below the vertebrarterial canal, on the posterior face of the vertebra, is a large oval facet for articulation with the corresponding surface of the axis, and on the upper surface of the centrum is a smaller facet corresponding to the surface on the ventral face of the odontoid; on the anterior surface are large, paired, irregularly crescentic facets for the occipital condyles (\$34).

27. In an entire skeleton note the mode of articulation of the vertebræ: the centra are united by pads of fibrocartilage, the intervertebral ligaments, only to be seen in the fresh state; the arches are united by the anterior zygapophysis of any vertebra being overlapped by the posterior zygapophysis of the vertebra next in front.

28. The intervertebral foramina, seen in the entire 
skeleton as spaces between the peduncles of successive neural arches; they serve for the transmission of the spinal nerves.

V. Make out, in the ribs and sternum :

29. The seven true ribs : each consists of a completely ossified vertebral rib dorsally, and of a cartilaginous sternal rib ventrally. The vertebral rib has a head articulating with the capitular facet on the centrum of its own vertebra and of that next in front; a tubercle on the dorsal side articulating with the tubercular facet on the transverse process of its own vertebra, and a short dorsal process just external to the tubercle. The sternal ribs, with the exception of the first, sixth, and seventh, articulate at the junction of two of the segments of the sternum.

30. The five false ribs : in all but the first of these the tubercle is obsolete, and in all the sternal rib does not articulate directly with the sternum.

3I. The sternum, consisting of six segments, the so-called sternebræ, of which the first is large, and keeled ventrally, and is called the manubrium. With the sixth is connected a rounded plate of cartilage, the xiphisternum or xiphoid process.

VI. In the skull note:

32. The large development of the facial as compared with the cranial portion of the skull, the latter including those bones which surround the brain-case.

33. The large foramen magnum, on the posterior surface of the brain-case, through which the spinal cord becomes continuous with the brain.

34. The two oval occipital condyles, bounding the foramen magnum at the sides and below.

35. The large hollow on each side of the skull formed mainly by the orbit or cavity for the eye, and bounded 
below by a bony bar, the zygoma: its posterior part answers to the temporal fossa.

36. The auditory aperture on each side of the hinder region of the brain-case: it is formed by a sort of bony tunnel, and looks upwards, outwards, and backwards.

37. The nasal apertures, quite in front of the snout, and confluent in the dry skull.

38. The very small extent of the bony palate, which is reduced to a narrow bridge running between the two rows of grinding teeth (see $\$ \S 65$ and 67 ).

39. The articulation of the lower jaw to the skull directly, and not through the intermediation of a quadrate bone.

40. The distinctness of most of the bones even in adult life.

4I. The long, slender processes, given off from many of the bones and helping, in the dry skull, to keep the various bones together.

42. The basioccipital, a flattened bone, bounding the foramen magnum below, and forming the hinder part of the base of the skull and the lower third of the occipital condyles.

43. The exoccipitals, joined below with the basioccipital, bounding the foramen magnum at the sides, and forming the upper two-thirds of the occipital condyles. Each is produced downwards into a paroccipital process, which fits closely against the posterior surface of the bulla tympani ( $\S 54$ ), and is perforated near its junction with the basioccipital by two small apertures, the condylar foramina, which transmit the hypoglossal nerve.

44. The supraoccipital, articulated below into the exoccipitals, and bounding the foramen magnum above : it is a very irregular bone, with a strongly-pitted surface, and marked externally by a median, escutcheon-shaped elevation. 
In the adult, the basi-, ex-, and supraoccipitals are all fused together into the single occipital bone.

45. The interparietal, a small transversely oval bone, interposed between the middle part of the front edge of the supraoccipital and the parietals $(\$ 46)$.

46. The parietals, a pair of thin, slightly arched bones, forming a considerable part of the roof of the brain-case : they are separated from one another by the median sagittal suture, and from the interparietal and supraoccipital, with which they articulate posteriorly, by the transverse lambdoidal suture : the outer edge of each gives off a long downwardly directed process, which, in the entire skull, is covered by the squamosal ( $\$ 53)$.

47. The frontals, roofing in the anterior part of the brain-case, articulated with one another in the middle line by the frontal suture, and with the parietals behind by the transverse coronal suture. Each is an irregular bone, with a slightly arched upper surface; an irregular anterior border; an orbital process, a thin plate continued downwards and inwards from its outer border, and forming the upper part of the inner wall of the orbit; and a supraorbital process, projecting directly outwards from the outer border, free at both ends, and forming a protection to the orbit above.

48. The basisphenoid, articulated behind by a broad surface with the basioccipital, and tapering in front to a blunt point; it is perforated at about the middle of its extent by an oval foramen, and its upper surface is hollowed out to form the sella turcica, which lodges the pituitary body $(\$ 484)$, and is bounded before and behind by the anterior and posterior clinoid processes, of which the latter only are formed by the basisphenoid.

49. The alisphenoids: two wing-like bones directed 
upwards and outwards, firmly ankylosed, even in young rabbits, one to either side of the basisphenoid, and produced below on each side into two laminæ, which unite with one another along their anterior edges, forming a dihedral angle open behind. These form the pterygoid process of the alisphenoid: with the inner lamina the pterygoid bone ( $(68)$ articulates.

50. The sphenoidal fissure, a large notch on each side between the basisphenoid and alisphenoid; it transmits the third, fourth, and sixth nerves, as well as the first and second divisions of the fifth.

5I. The presphenoid, a laterally compressed bone, joined behind to the basisphenoid by synchondrosis (cartilaginous union), so that in the dry skull there is always a considerable interval between the two bones. It forms the inferior and anterior boundary of a large oval aperture which puts the two orbits in communication with one another, and both in communication with the braincase : this is the optic foramen, and transmits the optic nerves $(\$ 485)$. On its upper or cranial surface the presphenoid is produced behind into the two anterior clinoid processes : in front of the optic foramen it gives off two irregular outwardly diverging laminæ, which form part of the inner wall of the orbit, articulating above with the inferior border of the orbital process of the frontal.

52. The orbitosphenoids: two wing-like laminæ directed outwards and slightly backwards, projecting one from each side of, and completely ankylosed with, the pre sphenoid: they form the superior and posterior boundary of the optic foramen.

53. The squamosal: a bone of very irregular form, articulating above with the parietal and frontal, in front with the orbital process of the frontal and the orbito- 
sphenoid, below with the alisphenoid, and behind with the descending process of the parietal. From its outer face is given off the zygomatic process, a strong bar of bone which passes first outwards, then downwards, and lastly forwards, and which bears on the under surface of its outwardly directed portion an articular surface-the glenoid cavity-for the lower jaw. From the posterior edge of the squamosal a slender process is given off, which passes backwards and downwards against the surface of the periotic $(\S 56)$.

54. The tympanic, a bone constituting the osseous part of the auditory meatus. It consists of a tubular portion above, and of a swollen portion below, which projects on the under surface of the skull and articulates with the lateral edge of the basioccipital: this last is the bulla tympani. Both tubular and bulbous portions are incomplete on the inner face, a vacuity being left which is only filled up when the tympanic bone is applied against the periotic. At the junction of the tubular and bulbous portions the tympanic is produced on its inner surface into a curved flattened ridge, to which the tympanic membrane is attached ( $\$ 346)$.

55. On the posterior border of the tympanic, at the junction of its tubular and bulbous portions is a shallow groove: when the tympanic is in place this notch is converted by the periotic into a canal, the stylo-mastoid foramen, which transmits the seventh nerve. A notch in the postero-inferior region of the bone is also converted into a canal by the juxtaposition of the periotic, forming the bony portion of the Eustachian tube $(\S 35 \mathrm{I})$.

56. The periotic: a very irregular bone, articulating: with the exoccipital behind, the supraoccipital above, the descending process of the parietal in front, and the basi- 
occipital below : the tympanic is closely applied to its outer surface, the tympanic cavity being enclosed between the two bones: a process from its posterior edge fits over the groove just noticed in the tympanic, completing the stylomastoid foramen and helping to keep the two bones in position. On its outer surface, and therefore seen only when the tympanic is removed, are two small apertures, the anterior of which is the fenestra rotunda, the posterior the fenestra ovalis. On its inner or cranial surface is a large hemispherical depression, the floccular fossa, for the lodgment of the flocculus of the cerebellum ( $\$ 477$ ), and beneath this is a shallow depression, the internal auditory meatus, with two apertures in it for the transmission of the seventh and eighth nerves (\$\$ 491, 492).

57. The foramen lacerum medium: a large space between the periotic and alisphenoid : it transmits the third division of the fifth nerve ( $\$ 357)$.

58. The foramen lacerum posterius: a space between the periotic and the exoccipital, through which the ninth, tenth, and eleventh nerves leave the skull ( $\$ 493)$.

59. The mesethmoid: this is seen only in the disarticulated or bisected skull: it consists of two parts, the cribriform plate, riddled with numerous small holes for the passage of the olfactory nerves, and completing the antero-inferior portion of the wall of the brain-case, and of the lamina perpendicularis, a median vertical plate, which proceeds from the anterior surface of the cribriform plate into the nasal cavity. In the recent state the lamina perpendicularis is continued forwards by a cartilaginous plate, the septum nasi, and the two together separate the right and left nasal cavities from one another.

6o. Lying against the ventral border of the septum nasi are the paired scroll-like Jacobson's cartilages: they are attached in 
front to the septum and free behind, and are ensheathed in the palatine processes of the premaxille. The dorsal edge of the septum is produced on each side into a long, narrow, horizontal shelf, which underlies the nasal, and is continued in front into a narrow alinasal cartilage, which encircles the nostril and unites below with the antero-ventral region of the septum.

6r. The parethmoids, or ethmo-turbinals, two complicated folded bones, situated one on either side of the mesethmoid, to which they are ankylosed in the adult.

62. The vomer, a long bone, deeply grooved on its upper surface, the groove receiving the inferior edge of the lamina perpendicularis and septum nasi; in the adult it is ankylosed to the parethmoids.

63. The nasals, two large flat bones which roof in the nasal cavities, articulating with one another in the middle line, and with the frontals behind. The inferior surface of each nasal is produced into a very thin, pouch-like process of bone, the naso-turbinal.

64. The premaxillæ, a pair of irregular bones which form the anterior boundary of the snout. Each gives off a long nasal process from its upper and posterior edge, which passes backwards and slightly upwards, articulating with the external border of the nasal and with the anterior edge of the frontal; a palatine process from its inner and anterior region, which passes directly backwards, in contact with its fellow of the opposite side along the palate ; and a short, stout maxillary process, which passes directly backwards from its postero-ventral region and articulates with the maxilla in the premaxillo-maxillary suture; and presents, in its antero-inferior region, two sockets or alveoli for the incisor teeth ( $\$ 77$ ).

65. The maxillæ, two large and very irregular bones, which, together with the premaxillæ, form the framework of the upper jaw. Their outer surfaces are spongy; on their 
ventral surfaces are the alveoli for the premolar and molar teeth ( $\$ 79$ and 80 ) ; the inner and inferior edge of each gives off, opposite the first and second premolar teeth, a horizontal palatine process, which, articulating with its fellow of the opposite side, forms the anterior half of the bony palate. From the outer and posterior region of each a strong process is given off, which turns first outwards and then backwards, its upper edge articulating with the lower edge of the zygomatic process of the squamosal; in the new-born rabbit this process is a separate bone, the jugal or malar. A little above the first premolar tooth ( $\$ 79)$ is an aperture, the infra-orbital foramen, leading by a canal into the orbit, and transmitting the second division of the fifth nerve $(\$ 358)$.

66. The maxillo-turbinals, two thin scroll-like bones, which occupy the anterior portion of the nasal chamber.

67. 'The palatines, thin lamina of bone, which articulate in front with the maxillæ, above with the orbital processes of the presphenoid, and behind with the pterygoid and with the pterygoid process of the alisphenoid. From the inner and anterior region of each is given off, opposite the third premolar tooth, a horizontal, inwardly directed palatine process, which, articulating in the middle line with its fellow of the opposite side, and in front with the palatine process of the maxilla, forms the posterior half of the bony palate.

68. The pterygoid, a small, irregular plate of bone, articulating with the posterior elge of the palatine and with the inner lamina of the pterygoid process of the alisphenoid (§ 49): it ends ventrally in the free, backwardly curved hamular process.

69. The lacrymals, two small bones fitted, one on each side, into the anterior wall of the orbit, between the 
maxilla and frontal : they often fall out in the dry skull. Each is perforated, near its outer border, by a small aperture for the lacrymal canal.

70. In the longitudinal section of the skull note the cranial fossæ for the reception of the chief divisions of the brain. The hindmost of these, or cerebellar fossa, for the lodgment of the cercbellum ( $\$ 477$ ), is marked off from the cerebral fossa, for the cerebral hemispheres ( $\$ 480)$, by a sharp oblique ridge along the inner face of the periotic and by the junction of the parietal and supraoccipital; it is along this line that the tentorium ( $\$ 162)$ is attached. The small olfactory fossa, for the olfactory lobes $(\$ 483)$, is an offshoot of the anterior end of the cerebral, and is marked only by an inconspicuous ridge on the inner surface of the frontal.

7 I. The mandible, or lower jaw, consisting of two separate halves, or rami, which articulate with one another in front by a rough surface. Each ramus consists of a horizontal portion, bearing the sockets for the teeth, and of an ascending portion, which ends above in the longitudinally elongated condyle for articulation with the squamosal. Anteriorly the ascending portion gives off the plate-like, slightly incurved coronoid process. The postero-inferior portion, or angle of each ramus, is somewhat produced backwards, and gives off an inward shelf-like projection. On the inner surface of each ramus, at the junction of the horizontal and ascending portions, is the inferior dental foramen, consisting of a number of small apertures and transmitting the third division of the fifth nerve ( $\$ 357$ ).

Interposed between the condyle and the glenoid cavity is a concavoconvex plate of cartilage, the meniscus.

72. The hyoid, situated at the root of the tongue, 
anterior to the larynx (see $\$$ I 24, I 25, p. 289), and always detached in the prepared skull: it consists of a stout thick body, or basi-hyal, a pair of small anterior cornua, or cerato-hyals, and a pair of long, backwardly directed posterior cornua, or thyro-hyals (cerato-branchials).

VII. Carefully break away in an entire skull the outer wall of the tympanic bone, and make out the following bones : ${ }^{1}$

73. The malleus, a small bone, recognised at once by the bony process or manubrium, by which it is attached, in the recent state, to the tympanic membrane. It consists of a rounded head, from which the manubrium and other lesser processes are given off, and which presents a saddle-shaped surface for articulation with the incus.

74. The incus, articulating by a saddle-shaped surface with the malleus, and giving off two processes, the short crus and the long crus.

75. The os orbiculare, a minute disk of bone attached to the long crus of the incus.

76. The stapes, a stirrup-shaped bone, having its base inserted into the fenestra ovalis $(\$ 56)$, and articulated by a small knob on the summit of its arch to the orbiculare.

VIII. Observe the following points in the teeth :

77. The incisors, of which two are situated in each premaxilla, and one in each ramus of the mandible. The anterior upper incisors are long, curved, chisel-shaped teeth, covered with enamel on the anterior surface only, marked on the same surface with a deep longitudinal groove, and destitute of roots. The posterior upper incisors are small teeth, situated behind the corresponding anterior incisors. The lower incisors resemble the anterior upper

1 This may be conveniently done with the skull of a specimen used for dissection. See Secs. XLII-XLIII. p. 331. 
incisors, except that they are not grooved anteriorly. In the position of rest they fit against the posterior upper incisors.

78. The absence of canines, or teeth situated immediately behind the premaxillo-maxillary suture.

79. The premolars: three on each side of the upper jaw (in the maxilla), two on each side of the lower jaw, and separated by a long interval or diastema from the incisors. They are long curved teeth, devoid of fangs. The first tooth in the upper jaw is small and quite simple: the remaining two are marked externally with a longitudinal groove, separating two well-marked ridges: their crowns present two transverse grooves bounded by ridges. In the lower jaw the first premolar has two grooves and three ridges, the second resembles those of the upper jaw.

80. The molars, three on each side of each jaw, and closely resembling the upper premolars, except that the last in each jaw is small and simple.

IX. In the shoulder-girdle and fore-limb note :

8r. The scapula, a flat triangular bone, lying, in the natural position, external to the anterior ribs, its apex being over against the first rib, and the long axis, drawn from its apex to its base, inclined upwards and backwards. The base of the triangle consists, in the recent state, of a strip of cartilage, the supra-scapula; its apex is formed by the smooth concave glenoid cavity for the articulation of the humerus: immediately anterior to this latter is a small inwardly curved coracoid process, consisting in the young animal (six weeks) of two separate ossifications and representing the coracoid of the lower Vertebrata: of these ossifications the pre-axial goes mainly to form the coracoid process, the post-axial is a small nodule bearing a facet which forms the anterior part of the glenoid cavity. 
The anterior or pre-axial edge of the scapula is the coracoid border, its post-axial edge the glenoid border; a third or acromial border is constituted by the free edge of the large spine which projects from its external face; a fourth or supra-scapular border by its dorsal edge. Its external surface presents a pre-scapular fossa anterior to, and a post-scapular fossa posterior to, the spine: its internal surface constitutes the sub-scapular fossa. The free ventral end of the spine is the acromion; a small process, which projects from it backwards and downwards, is the metacromion.

82. The clavicle, a small, curved, rod-like bone, attached by fibrous tissue at one end to the sternum, at the other to the acromion.

Attached to the outer end of the clavicle is a small nodule of cartilage, the meso-scapular segment : attached to its inner or sternal end are two similar nodules, that nearest the clavicle is the procoracoid, the other the omosternum.

83. If the fore-limb be compared with that of a Lizard (p. I47 $\S \mathrm{X}$.), it will be seen that the altered position of the parts in the mammal is due to the following changes $:^{1}$ (r) the distal end of the humerus has become rotated backwards so as to bring the whole limb into a plane parallel with the median vertical plane of the body; the pre-axial border then becomes external, and the originally dorsal surface looks, on the whole, backwards: (2) the radius and ulna have become fixed in the position of pronation, that is, the distal end of the radius has rotated round the ulna carrying the hand with it: the consequence of this is that while the pre-axial border of the proximal end of the

1 The changes in position of the vertebrate limbs are best made out on the skeleton of a man or ape, in which the bones are articulated so as to allow of the natural movements, including pronation and supination, being performed. See Flower, Usteology of the Mammalia, p. 245 . 
fore-arm is, like that of the humerus, external, the same border comes to be internal in the distal end of the fore-arm and in the manus, the dorsal surfaces of which look, on the whole, forwards.

84. The humerus, a long bone consisting of a shaft and of two prominent extremities, and having its long axis directed from the glenoid cavity downwards and backwards, so that its originally dorsal surface looks upwards and backwards, its ventral surface downwards and forwards, its pre-axial border outwards, and its post-axial border inwards. The proximal extremity presents, dorsally, a rounded head for articulation with the glenoid cavity; pre-axially, a large projection, the greater tuberosity ; post-axially, a smaller projection, the lesser tuberosity; and between the tuberosities a depression, the bicipital groove. On the anterior (ventral) surface of the proximal end of the shaft is the deltoid ridge. The distal extremity presents a large pulley-like surface, or trochlea, for the articulation of the ulna and radius, and a smaller pre-axial surface, or capi. tellum, for the radius only (see $\S \S 85$ and 86 ); a deep olecranon fossa on the posterior (dorsal) side, for the reception of the olecranon process of the ulna, perforated by a small supra-trochlear foramen; a small pre-axial external condyle just outside the capitellum; and a larger post-axial internal condyle, similarly situated with regard to the trochlea. In the young rabbit the extremities of the humerus consist of separate bones, or epiphyses.

$8_{5}$. The radius, or shorter bone of the fore-arm, slightly curved forwards, and so situated that its true dorsal surface looks forwards and slightly upwards, and its pre-axial border inwards. Its proximal extremity or head presents a double articular surface for the pulleys of the humerus: its distal extremity, a pair of slight concavities for the 
scaphoid and lunar bones ( $\$ 87)$ : the ventral (posterior) surface of the shaft is flattened, and fits against the ulna. In the young animal both extremities consist of epiphyses.

86. The ulna, or longer bone of the fore-arm: it is immovably articulated, though not ankylosed, to the radius, in the position of pronation, its anterior surface being flattened for the reception of that bone. At the proximal end of the same surface is the sigmoid cavity for the articulation of the humerus: beyond this the bone is produced into the olecranon or anconeal process. At its distal end the ulna presents a convex surface for the articulation of the cuneiform ( $\$ 87)$. In the young animal the whole distal end of the bone is formed of an epiphysis: the proximal epiphysis is small, consisting of a nodule on the olecranon.

87. The carpus, consisting of a proximal and a distal row of small nodular bones. The bones of the proximal row, beginning from the pre-axial (inner) side, are scaphoid (radiale), and lunar (intermedium), articulating with the radius, cuneiform (ulnare), articulating with the ulna, and pisiform (a sesamoid), articulating with the ventral (palmar) side of the cuneiform and with the ulna. In the distal row are five bones, the central one of which is distinctly proximal to the other four, and really constitutes by itself a middle row; this is the centrale, the others being trapezium (carpale $\mathrm{I}$ ), on the pre-axial side, then trapezoid (carpale 2), magnum (carpale 3) situated immediately to the post-axial side of the centrale, and unciform (carpalia 4 and 5 ) on the post-axial side.

88. The metacarpus, consisting of the five metacarpal bones, of which that belonging to the first or preaxial digit (pollex) is the shortest, the fifth somewhat longer, 
the second and fourth still longer, and the third longest of all. The first articulates with the trapezium, the second with the trapezium, trapezoid, centrale, and magnum, the third with the magnum and unciform, and the fourth and fifth with the unciform. Their distal ends present pulley-like surfaces for articulation with the proximal phalanges, and in the young animal are formed by epiphyses: they have no proximal epiphyses.

89. The phalanges, of which there are two to the pre-axial digit, or pollex, and three to the other four. The proximal and middle phalanges have hollowed proximal and pulley-like distal articular surfaces; the distal or ungual phalanges have hollowed proximal surfaces, and have a conical form, the dorsal surface being grooved for the firmer attachment of the horny claw. In the young rabbit the proximal end of the proximal and middle phalanges are formed by epiphyses: they have no distal epiphyses.

90. The sesamoid bones, small nodular ossicles situated on the palmar side of the joints of the digits : there is a pair between the metacarpal and proximal phalanx, and a single one between the middle and distal phalanges of all the digits except the first ; and one between the proximal and distal phalanges of the pollex.

\section{In the hip-girdle and hind limb note :}

9I. The pelvis, or hip-girdle, consisting of the two innominate bones. Each of these consists of an antero-dorsal portion, the ilium, articulated by a rough surface to the sacrum, and of a postero-ventral portion perforated by a large aperture, the obturator foramen, which divides it into a pre-axial (anterior) part, the pubis, and a post-axial (posterior) part, the ischium. In the young animal the ilium, ischium, and pubis are separate bones meeting one another in a triradiate suture in the centre of 
the acetabulum, ${ }^{1}$ a deeply concave articular surface on the external face of the innominate bone. The ilium presents a ventral (pre-axial) pubic border, a dorsal (post-axial) ischial border, an external acetabular border consisting of an inconspicuous ridge running from the acetabulum along its outer surface, and an anterior (dorsal) supra-iliac border along its free end: that portion of its external surface on the ventral side of the acetabular border is the iliac fossa, that on its dorsal side the gluteal fossa; its inner surface is the sacral fossa. The pubes of opposite sides unite with one another in the middle line, by synchondrosis, to form the symphysis pubis : the posterior part of the ischium is expanded and roughened, forming its tuberosity (tuber ischii).

92. If the hind-limb be compared with that of the Lizard (p. I5I $§$ I08), it will be seen that the femur, and with it the whole limb, has undergone rotation forwards : as a consequence of this the hind-limb is, like the fore-limb, brought into a plane parallel with the median vertical plane of the body, but, the rotation being in the opposite direction, the pre-axial border is internal in the whole limb, and the originally dorsal surface looks, on the whole, forwards.

93. The femur, the long axis of which, in the natural position, is directed downwards and forwards, so that its originally dorsal surface looks upwards and forwards, and its pre-axial border inwards. Its proximal extremity presents a rounded, projecting head on its pre-axial (inner) border, for articulation with the acetabulum; a large process, the greater trochanter, just external to the head; a pit, the trochanteric fossa, on the ventral surface, bounded pre-axially by the great trochanter; a lesser trochanter

1 The pubis proper is really shut out from the acetabulum by a small epiphysis, the cotyloid bone. 
on the pre-axial border, immediately distal to the head; and a small third trochanter on the post-axial (external) border, slightly beyond the great trochanter. On the head is a shallow pit for the attachment of the ligamentum teres $(\S 47 \mathrm{I})$. Its distal extremity presents an internal condyle (pre-axial) and an external condyle (postaxial), separated from one another by the intercondylar notch, which, with the condyles, forms the articular surface for the tibia ( $(96)$ and patella ( $(95)$. The internal and external tuberosities are small roughened projections, situated, the first on the pre-axial, the second on the post-axial, border of the distal extremity of the femur, close to the condyles. In the young animal the whole distal end of the femur is formed by an epiphysis, while at the proximal end are two epiphyses, one forming the head, the other the greater trochanter.

94. Two nodular sesamoid bones, or fabellæ, occur in connection with the distal end of the femur, a smaller preaxial, and a larger post-axial, both being situated quite on the posterior (ventral) aspect of the bone.

95. The patella (a sesamoid), a small nodule of bone, fitting against the pre-axial end of the intercondylar notch, and connected by ligament to the tibia.

96. The tibia, or larger bone of the shank, so placed that its true dorsal surface looks forwards and slightly downwards, and its pre-axial border inwards. Its proximal extremity presents two slightly concave articular surfaces for the condyle of the femur, and two roughened tuberosities on its pre-axial and post-axial borders respectively. A prominent ridge, the crest of the tibia, extends along the proximal end of the (anterior) dorsal surface of the bone. Its distal extremity presents two articular surfaces, one pre-axial, and pulley-like, for the astragalus, the other post- 
axial, somewhat flattened, and projecting beyond the first, for the calcaneum ( $\$ 99)$.

97. The femoro-tibial or semilunar cartilages, two thin plates interposed between the femur and the tibia: their concave edges are turned towards one another, and they are situated immediately beneath the condyles of the femur.

98. The fibula, a small rod of bone, attached by its proximal end to the outer tuberosity of the tibia; distally it fuses with the tibia at the middle of its length ; its proximal end is formed by an epiphysis.

99. The tarsus, consisting of six irregular bones arranged in three rows. In the proximal row are two bones, the astragalus (tibiale), situated on the pre-axial side, and having at its proximal end a large pulley-like surface for articulation with the tibia ; and the calcaneum (fibulare), on the post-axial side, having at about the middle of its length an articular surface for the tibia, beyond which it is produced into the long calcaneal process, or heel. In the middle row is the single navicular (centrale), articulating chiefly with the distal end of the astragalus, but partly also with the distal and pre-axial edge of the calcaneum; its ventral or plantar surface is produced into a large distally directed process. In the distal row are three bones, the mesocuneiform (tarsale 2 ) and ectocuneiform (tarsale 3 ), articulating with the navicular, and the cuboid (tarsalia 4 and 5), articulating chiefly with the calcaneum. The entocuneiform (tarsale I), is either absent or is ankylosed with the proximal end of the second metatarsal (see $\S$ roo).

100. The metatarsals, four in number, the first (that of the hallux or pre-axial digit) being absent. The third is the longest, the fourth the next longest, and the second and fifth of about the same length. The proximal end of the second (the apparent first) articulates with the mesocuneiform, 
and is produced into a process which passes external to the latter, and articulates with the navicular; this process is a separate bone in the young rabbit, and may represent the entocuneiform. The second metatarsal articulates with the ectocuneiform, the third and fourth with the cuboid : all four are in close contact with one another at their proximal ends : their distal ends are pulley-shaped.

ror. The phalanges, of which each digit has three: they have the same characters as those of the manus $(\S 89$. p. 282).

102. The sesamoid bones of the pes are similarly situated to those of the manus.

\section{B.-DIRECTIONS FOR DISSECTION.}

XI. Make out the external characters as follows:

103. The hairy integument, which invests the body, the hairs extending even over the tip of the nose and the walking surfaces of the feet.

I04. The division of the body into head, trunk, and limbs: the union of the head and trunk by a distinct though short neck: the short tail.

105. The division of the head into a hinder cranial portion containing the brain, and an anterior facial portion or snout.

ıо6. The external nostrils or anterior nares, oblique slits at the fore end of the snout, connected by a median hairless groove with the mouth.

I07. The mouth, a small transverse aperture bounded by the upper and lower lips; the hairy integument stops at the edges of these above and below, while at the sides it is continued for a considerable distance into the cavity of the mouth.

ı08. The vibrissæ, or "whiskers," a number of long stout bristles on the upper lip. 
I09. The large eyes, guarded by an upper and a lower eyelid, and by a third eyelid or nictitating membrane, which can be pulled over the eye from its anterior (inner) corner, and is strengthened by a plate of cartilage.

I I. The auditory apertures, one on either side of the hinder region of the head, guarded by the large external ears.

III. The two rami of the mandible can be felt through the skin, and, between their hinder ends, the movable $\operatorname{larynx}\left(\mathrm{I}_{24}\right)$.

II2. The vertebral column can be felt extending from just behind the head to the extremity of the tail.

I I3. At the sides of the anterior part of the trunk, the ribs can be felt through the skin, and along the middle ventral line of the same region the breast-bone or sternum. These. with the corresponding part of the vertebral column, enclose the anterior or thoracic region of the trunk.

I I 4. The posterior or abdominal region of the trunk, enclosed at the sides and below only by skin and muscle, except at its posterior extremity, where it is bounded by the pelvis, the ventral portion of which, or symphysis pubis, can be readily felt through the skin.

II5. The anus, a rounded aperture just under the root of the tail, and bounded laterally by two naked patches of integument, the perineal spaces, on which open the ducts of the perineal glands (see $\$ 277$ ), to the secretion of which the animal owes its peculiar and unpleasant smell.

I 6 . The urinogenital aperture, between the anus and the symphysis pubis : in the male it is small and situated at the extremity of the backwardly directed intromittent organ or penis, in the female it is a large slit-like opening or vulva. 
I 7 . In the adult male, a pair of considerable projections will be seen, one on either side of the root of the penis; these are the scrotal sacs, and contain the testes.

I 8 . The scapula, a large bone, attached to the rest of the skeleton only by muscle, can be felt through the skin in the antero-dorsal region of each side of the thorax.

I 9. The division of the fore-limb into upper-arm or brachium (almost hidden by the skin), fore-arm or antebrachium, and hand or manus, the latter provided with five clawed digits : of these the innermost or pre-axial digit is distinguished as the thumb or pollex.

I20. The division of the hind-limb into thigh or femur (almost hidden in the skin), shank or crus, and foot or pes, the latter very long, and terminated by four clawed digits: the innermost of these corresponds with the index, the true first digit or hallux being absent.

I2 1 . In the female, the teats of the four or five pairs of mammary glands are seen, about two inches apart, on either side of the middle ventral line of the body: the anterior pair is thoracic, and occurs at about the junction of the anterior and middle thirds of the thorax : the remaining three pairs are abdominal in position.

XII. Fix the animal in the supine position, i.e., with the ventral surface upwards ; make a longitudinal incision into the skin over the sternum, and continue the cut forwards to the mandibular symphysis and backwards to the pubic symphysis. With the handle of a scalpel, carefully separate the skin from the underlying parts over the whole ventral surface. Note:

122. A thin sheet of muscle-the panniculus carnosus, or cutaneous muscle-lying immediately beneath the skin, to which it is closely attached, and covering the 
whole ventral surface of the trunk and neck; it is readily distinguished from the skin by containing numerous bloodvessels. In all probability it will be removed with the skin, if not, it should be dissected off separately.

The cervical portion of the panniculus is called the platysma myoides : the fibres of its thoracic portion are gathered into a strong bundle, which becomes tendinous and is inserted into the shaft of the humerus at its proximal end.

I23. In the adult female, the mammary glands, large, irregular, whitish masses, situated between the skin and the underlying muscles, and opening externally by the teats.

I24. The trachea, or windpipe, in the middle line of the neck, known by its regularly arranged cartilaginous rings; it ends in front in the larynx, which is situated in the hinder end of the triangular space inclosed by the two rami of the mandible: to see these and the following structures clearly the cervical portion of the cutaneous muscle should be carefully dissected away.

125. A meciian mass of muscle (mylohyoid, \&c.) situated just anterior to the larynx and between the anterior ends of the mandibular rami: in it the hyoid bone is embedded; the posterior cornua $(\$ 72)$ emerge from its hinder border and pass backwards and outwards towards the larynx.

I26. The submaxillary (salivary) glands, reddish, ovoidal bodies, about one-third inch in length, situated one on either side of the middle line, somewhat anterior to the larynx.

I27. The external jugular veins, large vessels, usually gorged with blood after death, lying one on either side of the trachea. 
I28. The sternum, a bony bar in the middle line of the chest, ending behind in a large discoidal cartilage, the xiphoid process.

I 29. The small, incompletely ossified clavicles, passing each from the upper extremity of the sternum to the corresponding shoulder.

I30. The sterno-mastoid, a long slender muscle, arising in conjunction with its fellow of the opposite side from the anterior end of the sternum, and passing obliquely forwards and outwards to the skull, just internal to the external jugular vein.

I3I. The pectoralis major, a large muscle, covering the ventral wall of the thorax : its fibres pass from the side of the sternum upwards and outwards to the upper arm.

The fibres of the pectoralis major pass either directly outwards, or, in the posterior part of the muscle, ontwards and forwards, and are gathered into a strong bunalle which is inserted into the deltoid ridge of the humerus.

132. The pectoralis tenuis, a thin sheet of transverse fibres covering the anterior part of the pectoralis major.

133. The pectoralis minor, seen by removing the pectoralis major and tenuis : it is a triangular muscle arising from the sternum, and inserted partly into the clavicle, partly into the scapula (see $§ 40 \mathrm{I}$ ).

134. The ribs, largely concealed by the pectoral muscles; each consists of a bony portion (vertebral rib) attached to the vertebral column, and of a cartilaginous portion (sternal rib), attached to the sternum: they are best seen by removal of the pectoral muscles.

135. The external intercostal muscles, short fibres filling up the interspaces between successive ribs: they are very transparent in the fresh subject, allowing the pink lungs to be seen through them.

I36. The blood-vessels and nerves of the arm, 
passing from the thorax dorsal to (beneath in the present position) the pectoral muscles, outwards to the arm.

I37. The linea alba, a faint band of fibrous tissue, more opaque than the general muscular walls of the body, passing from the posterior extremity of the sternum to the symphysis pubis.

I38. The rectus abdominis, a band-like area of muscle lying on either side of the linea alba: its fibres run longitudinally.

I39. The external oblique muscle, just external to the rectus : its fibres run from above downwards and backwards.

I40. The internal oblique, seen by dissecting away part of the external oblique, which it immediately underlies : its fibres run downwards and forwards, crossing those of the external oblique.

I41. The transversalis, seen by dissecting away a portion of both external and internal oblique : it lies between the latter and the peritoneum (\$ 145 ), its fibres running nearly parallel to those of the external oblique.

142. Poupart's ligament, a fibrous cord passing on each side from the symphysis pubis upwards and forwards to the anterior part of the ilium (\$9r).

I43. The blood-vessels and nerves of the legs, passing from the abdominal cavity, beneath Poupart's ligament, outwards to the leg.

I44. The abdominal viscera are seen, especially in young subjects, through the thin abdominal walls.

XIII. Make an incision down the linea alba, from the xiphoid process to the symphysis pubis : from the anterior extremity of this, make transverse incisions, and turn back the flaps of muscle. Without disturbing anything, note in the abdominal cavity thus laid open :

145. The peritoneum, a moist shining membrane, lining the whole abdominal cavity. 
I 46. The diaphragm, a thin membrane, partly muscular, partly fibrous, which forms the anterior wall of the cavity: through its transparent central portion will be seen the pink lungs.

I 47. The large, dark-red liver, made up of several lobes, and situated immediately posterior to the diaphragm.

I48. The smooth-walled, bluish-white stomach, partly covered by the liver : its more dilated (cardiac) end is seen to lie towards the animal's left side.

I 49. The reddish coils of the small intestine, some of which are seen at the anterior, others at the posterior part of the abdominal cavity.

I 50 . In about the posterior two-thirds of the cavity are seen (usually) three folds of the enormous cæcum or blind gut, known by its olive-green colour, the spiral constriction encircling it, and the fact of its diameter being many times greater than that of the small intestine.

I $5 \mathrm{~J}$. The colon, usually situated between two folds of the cæcum, with which it is seen to be connected, and which it resembles in colour; its diameter is less than that of the cæcum, and its walls are sacculated.

152. In the hinder end of the cavity, as well as in its right anterior corner, are usually to be seen portions of the rectum, which is of a light brownish colour, and contains pill-like masses of black fæcal matter.

I53. Quite at the posterior end of the cavity is seen the urinary bladder, the amount visible of which varies greatly according to its state of distension

I54. In a male specimen the scrotal sacs, hanging down from the abdominal cavity, one on each side of the penis.

I 5.5. By turning aside the intestines, the kidneys ( $\$$ I 87 , r96) are seen attached to the dorsal wall of the abdomen. 
I56. In the female the uteri ( $\left.\S_{2} 88\right)$ and ovaries ( $\$ 291$ ) are seen when the intestines are turned aside: they are inconspicuous structures in young individuals, but very obvious in adults.

XIV. Make a small aperture in one side of the diaphragm - say the left-and note the immediate collapse of the left lung. Dissect away the pectoral muscles, cut through all the vertebral ribs of the left side, except the last five, at about a quarter of an inch from their junction with the sternal ribs: from the posterior end of the incision thus made, cut downwards (i.e. towards the sternum) for about an inch, and then forwards, cutting through the sternal ribs: turn forwards the flap thus separated, and carefully clissect it away from the underlying tissues at its anterior end, so as to detach it altogether without injuring the jugular and brachial veins: proceed in the same way on the right side, noting that the right lung, like the left, does not collapse as long as the thoracic walls of its own side are intact. Note in the thoracic cavity thus laid open :

157. The small size of the thoracic as compared with the abdominal cavity : this is usually the case in herbivorous animals.

I58. The heart, a dark-red, conical body in the centre of the thoracic cavity, and enclosed in a thin membrane, the pericardium: its broad base is turned forwards, its blunt apex points backwards and somewhat to the left side.

I 59. The thymus gland, a soft, fat-like body, anterior to, and partly hiding the base of, the heart. It is especially large in young animals. 
I60. The lungs, spongy bodies of a light pink colour, situated one on either side of the heart. 'They will be, by the pressure of the air on their outer surfaces, completely collapsed: to see their actual shape and relations they should be inflated from the trachea, by making a small aperture in it and inserting a blowpipe.

I6r. The pleura, a shining membrane lining the thorax and forming a double median vertical partition - the mediastinum-which divides the thorax into right and left pleural cavities, in each of which a lung is contained : the mediastium is seen in the space between the pericardium and the sternum (see $\$ 222$ ).

$X V$. Place the animal in the prone position, i.e. with the dorsal surface upwards, first pinning together the reflected abdominal walls to keep the viscera in position : riake a median incision through the skin from the snout to about the middle of the thoracic region, and dissect away the skin from the whole dorsal surface of the head and neck. Dissect away the muscle from the anterior region of the neck and posterior region of the head, and find the occipito-atlantal articulation, or place where the skull articulates with the vertebral column. Cut through the occipito-atlantal membrane, which will be seen stretching between the supraoccipital and the arch of the atlas: the anterior end of the spinal cord will be seen, arched over in front of the space by the occipital bone, behind by the atlas vertebra. Insert the point of a pair of bone-forceps or strong scissors between the cord and the bone, and cut away the arch of the atlas: do the same with the arches of the two or three succeeding vertebræ, 
until a small portion of the cord is exposed; then, inserting the forceps between the medulla oblongata and the occipital bone, remove the roof of the skull and expose the brain. Great care will be required to do this without injuring the brain, especially in the region just internal to the external auditory opening, where a lobe of the cerebellum (see $\$ 477$ ) will be found embedded in a deep pit of the skull wall. Note as you proceed :

162. The dura mater, a tough fibrous membrane lining the cranial and spinal cavities: it is continued inwards between the cerebral hemispheres ( $\$ 480$ ) as the falx cerebri, and between the cerebrum and cerebellum as the tentorium: it is sometimes torn away with the fragments of bone, sometimes left adhering to the brain: in the latter case it is advisable to dissect it away from the dorsal surface of the organ before attempting its removal.

I63. The pia mater, a delicate shining membrane closely investing the brain and cord, and abundantly supplied with blood-vessels.

I64. As in the Pigeon (p. $22 \mathrm{I}, \S \mathrm{XXV}$.), the brain completely fills the skull cavity, the space between the dura mater and pia mater being reduced to a minimum.

XVI. Cut through the spinal cord about a quarter of an inch beyond its junction with the brain: carefully lever up the brain with the handle of a scalpel, and cut through all the nerves proceeding from it, working from behind forwards: dissect away the olfactory lobes $(\$ 483)$ from their attachments. Remove the whole brain and place it in alcohol, or, better, in saturated solution of zinc chloride, 
for a day or two, and then, after removal of the pia mater, in strong alcohol (see note, p. 46).

XVII. Place the animal again in the supine position, and make out in the abdominal cavity, without cutting or tearing anything:

I65. The characters of the liver: its convex anterior surface applied to the diaphragm, its concave posterior surface against which the stomach fits, its attached dorsal and free ventral borders. The dorsal border is attached by a thin sheet of peritoneum, the coronary ligament, to the dorsal part of the posterior surface of the diaphragm: a median vertical sheet of peritoneum, the suspensory ligament, connects the anterior surface of the liver with the posterior surface of the diaphragm. This latter ligament divides the liver into a right and left segment; each segment is again divided into lobes, of which there are, on the right side, the ventral right central lobe (Fig. 6o, p. $301, r . c)$, and the dorsal caudate lobe (caut) closely applied to the right kidney; and on the left side, the ventral left central lobe (l.c), the dorsal left lateral lobe (l.l) fitting against the left anterior region of the stomach, and the small Spigelian lobe $(s p g)$, fitting closely into the lesser curvature (see $\S$ r68) of the stomach.

I66. The gall bladder, a green pyriform sac, embedded in the right central lobe of the liver.

I67. The œsophagus, or gullet, a narrow tube emerging from the thorax through an aperture in the diaphragm, dorsal to the liver, and entering the stomach.

168. The stomach, divided into a large cardiac portion to the animal's left, and a smaller pyloric portion to the right side; its posterior greater curvature, and anterior lesser curvature; the cardia or entrance of the œsophagus towards the left of the lesser curvature, and the 
great cardiac dilatation of the stomach to the left of this point; the pylorus, or place of exit of the duodenum ( $(\mathrm{I} 73$ ), at the pyloric end; and the antrum pylori, a somewhat dilated and thickened portion of the stomach, immediately to the left of the pylorus.

I69. The gastro-hepatic omentum, a sheet of peritoneum connecting the posterior surface of the liver with the lesser curvature of the stomach, and covering the Spigelian lobe. It is continuous, towards the right side, with the duodeno-hepatic omentum, which connects the surface of the liver with the proximal end of the duodenum (\$ I 73 ).

I 70. The mesogaster, or sheet of peritoneum connecting the stomach with the dorsal wall of the abdominal cavity.

I 7 r. 'The great omentum, a double fold of peritoneum, connected with the greater curvature of the stomach, and usually loaded with fat.

I 72. The spleen, a long, flat body of a dark red colour, attached to the cardiac dilatation of the stomach by a sheet of peritoneum, the gastro-splenic omentum.

I73. The dúodenum, or portion of the small intestine immediately succeeding the stomach; it forms a long U-shaped loop, not bound up with the rest of the small intestine, but closely connected with a portion of the rectum.

I74. The arrangement of the remainder of the intestine: the cæcum with the greater part of the colon and part of the rectum are bound together by a single fold of peritoneum, the mesentery, which also suspends them to the dorsal wall of the abdomen: the greater part of the ileum, or portion of small intestine intervening between the duodenum and the cæcum, is supported by a separate fold of mesentery.

175. The connection of the lower end of the rectum 
to the dorsal body-wall by a sheet of peritoneum, the mesorectum.

I 76. The passage of the ileum into the proximal end of the cæcum, and the exit of the colon from the same.

I77. The termination of the distal or blind extremity of the cæcum in a finger-like, thick-walled process, about three inches long, the appendix vermiformis.

I78. The passage of the rectum posteriorly into the pelvic cavity, between the bladder and the vertebral column, to open externally by the anus.

I79. The reflections of the peritoneum : its parietal layer, or lining of the abdomen, on reaching the middle dorsal line is reflected ventralwards, forming the various subdivisions of the mesentery-mesentery proper, mesogaster, mesorectum, \&c. - which thus consists of two closely applied layers, one continuous with the parietal layer of the right, the other with that of the left, side : on reaching the alimentary canal the two layers diverge again and invest it, forming the visceral layer or peritoneal investment of the alimentary canal.

XVIII. Turn over the stomach and intestines to the animal's right side, and make out :

โ80. The postcaval vein, or inferior vena cava ${ }^{1}$

1 A rabbit is best injected for anatomical purposes with plaster of Paris (see p. 48, note). Kill the animal with chloroform or potassic cyanide : as soon as it is dead, open the thorax by cutting through the sternal ribs of both sides, sufficiently far fron the middle line not to injure the mammary arteries ( $\$ 250$ ), cutting across the posterior end of the sternum and turning it forwards: slit open the pericardium, and make a large incision, by a single cut of the scissors, in each ventricle: all this should be done very rapidly, if possible before the heart has ceased to beat, as it is desirable to get rid of as much blood as possible: pass a ligature round the aorta close to its exit from the heart, and give it a single loose tie: when the bleeding has ceased, sponge the blood from the heart, and pick out any clots which may have formed in the left ventricle : pass a cannula through the incision in the left ventricle into the aorta, tighten the ligature and knot it firmly. Bythis operation 
(Fig. 6I, ptc), a large vessel, usually gorged with dark blood after death, passing from the pelvis forwards along the ventral aspect of the backbone, through a notch in the liver, to the diaphragm.

I 8I. The aorta (Figs. 60 and 6r, d.ao), a smaller vessel than the postcaval but with thicker walls, of a pinkish colour, and usually containing comparatively little blood after death ; it runs partly behind, partly alongside, the postcaval.

I 82. The cœliac artery (ca.a), given off from the aorta about an inch posterior to the diaphragm, and supplying the stomach, liver, spleen, and proximal parts of the duodenum (see $\S$ I98).

I83. The anterior mesenteric artery (a.m.a), arising from the aorta about half or three-quarters of an inch posterior to the coliac: it supplies the greater part of both small and large intestine.

IS4. The cœliac ganglion, a soft, pinkish-white mass of irregular form, situated just anterior to the origin of the anterior mesenteric artery : it is connected by nerres to the mesenteric ganglion, which lies immediately posterior to the artery.

I 85. The splanchnic nerve, emerging from beneath the diaphragm and passing backwards across the aorta to join the cœliac ganglion : it is a branch of the sympathetic ( $\$ 239$ ).

I86. The gastric branch of the vagus, a small nerve taking a somewhat spiral course round the gullet to the stomach : branches from it join the cœliac ganglion.

I87. The left kidney, a brownish-red body of characteristic shape, just posterior to the anterior mesenteric artery,

the whole of the systemic arteries are injected : the pulmonary arteries may be filled by proceeding similarly on the right side. The portal vein is readily injected from its branch to the caudate lobe (Fig.6o, cau.), the cannu'a being directed towards the main trunk. The injection of the systemic veins is more difficult : the precavals can be filled from the external jugular, the postcaval from the external iliac, the cannula, in both cases, being directed towards the heart. 
and connected by the renal artery and vein with the aorta and inferior cava respectively (see $\$ \$ 253$ and 254).

I88. The left ureter, a delicate white tube, passing from the inner edge of the kidney backwards and inwards towards the pelvis : its exact relations will be studied later (\$ 262).

I89. The left adrenal or suprarenal body, a small, rounded, yellowish body, placed immediately anterior to the origin of the renal artery and vein.

190. In the female the left uterus, Fallopian tube, and ovary are now displayed, but are best studied at a later stage ( $\$ 288-29 \mathrm{I})$.

I9I. The posterior mesenteric artery (p.m.a), a small vessel leaving the aorta a short distance posterior to the left kidney and branching out in the mesorectum to supply the rectum.

I 92. The posterıor mesenteric vein (Fig. 6o, p.m.v), a small vessel in the mesorectum formed by the union of branches parallel with those of the artery of the same name : it passes directly forwards to join the anterior mesenteric vein (§ I 93).

XIX. Turn the intestines over to the animal's left side, and spread out the duodenum, putting its mesentery slightly on the stretch, but taking care not to rupture it (see Fig. 60). Note :

193. The anterior mesenteric vein $(m . v)$, a large vessel usually gorged with blood after death, lying in the duodenal mesentery and receiving veins from the various coils of the intestine; it is the chief factor of the portal vein (§ I99).

I 94. The pancreas ( $p n)$, not forming a solid mass, but consisting of a number of small lobules, looking not unlike fat, spread all over the duodenal mesentery.

I95. The pancreatic duct ( $p n . d)$, a short delicate tube, 


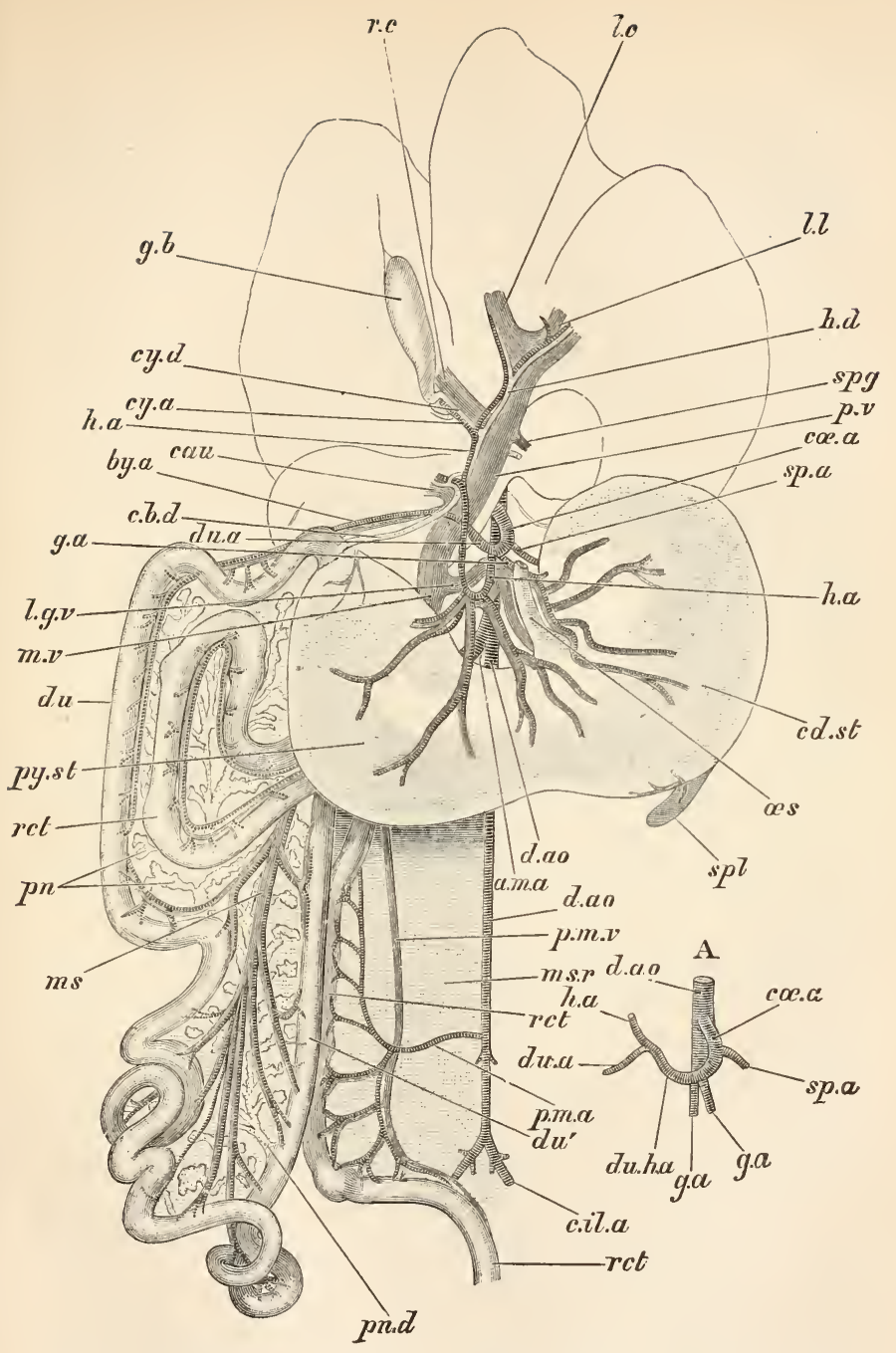

FIG. 6o.-Lepus cuniculus. The stomach, duodenum, posterior portion of rectum, and liver (in outline), with their arteries, veins, and ducts. A. The cœliac artery of another specimen (both $\times \frac{2}{3}$ ). 
The guilet ( $\propto s)$ is cut through and the stomach somewhat displaced backwards to show the ramifications of the coliac artery $(c \alpha . a)$ : the duodenum $(d u)$ is spread out to the right of the subject to show the pancreas $(p n)$ : the branche; of the bile-duct $(c . b . d)$, portal vein $(p . v)$, and hepatic artery $\left(h_{0} a\right)$, are supposed to be traced a short distance into the various lobes of the liver.

a.m.a, anterior mesenteric artery : cau, caudate lobe of liver, with its artery, vein, and bile-duct : c.b.d, common bile-duct : $c d . s t$, cardiac portion of stomach : c.il.a, common iliac artery : co.a, cœeliac artery: $c y . a$, cystic artery $: c y . d$, cystic duct : $d . a o$, dorsal aorta : du, proximal, and $d u^{\prime}$, distal, limbs of duodenum: $d u \cdot a$, duodenal artery: $d u \cdot h \cdot a$ (in A), duodeno-hepatic artery : g.a, gastric artery and vein : $g . b$, gall bladder : $h . a$, hepatic artery : $h . d$, left bile-duct $: l . c$, left central lobe of liver, with its artery, vein, and bile-duct: l.g.v, lieno-gastric vein : l.l, left lateral lobe of liver, with its artery, vein, and bile-duct: $m s$, branch of mesenteric artery and vein to duodenum : ms.r, mesorectum : $m . v$, chief mesenteric vein : as, œesophagus : p.m.a, posterior mesenteric artery : $p . m . v$, posterior mesenteric vein: $p n$, pancreas : $p n . d$, pancreatic duct : $p . v$, portal vein : py.st, pyloric portion of stomach : rct, rectum : r.c, right central lobe of liver, with its artery, vein, and bileduct : $s p g$, Spigelian lobe of liver, with its artery, vein, and bileduct : spl, spleen : sp.a, splenic artery.

opening into the ascending or distal limb of the duodenal loop, an inch or so beyond its junction with the descending or proximal limb; the duct will be seen to branch out into a number of fine tubes which pass to the lobules of the gland.

I96. The right kidney, situated about an inch farther forward than the left kidney, and having its anterior portion partly covered by the caudate lobe of the liver; the right adrenal is in contact with its inner border, immediately anterior to the right renal artery and vein, and the right ureter passes from its inner edge backwards to the pelvis.

$\mathrm{XX}$. Turn the intestines into their natural position again ; tie a double ligature round the gullet close to the diaphragm and cut it between the ligatures; draw the stomach backwards, turn forward the lobes of the liver, and dissect out the following structures (see Fig. 60) : 
197. The common bile-duct (c.b.d), a delicate transparent tube containing greenish bile, entering the duodenum just beyond the pylorus, and receiving hepatic ducts from the various lobes of the liver and a cystic duct from the gall bladder.

Calling the varic us hepatic ducts by the same names as the lubes from which they spring, the arrangement of the whole series of biliary ducts is as follows :-The cystic duct $(c y . d)$ unites with the right central duct to form a right bile-duct : the left central and left lateral ducts similarly unite to form a left bile-duct $\left(h_{0}(d)\right.$ : the right and left bile-ducts unite to form the common bile-duct (c.b.d), which, about half way between its commencement and its termination in the duodenum, receives the posterior bile-duct formed by the union of caudate and Spigelian ducts. They are best demonstrated by injection from the duodenal aperture.

198. The branches of the cœliac artery ( $(1 \& 2)$ : shortly after its origin from the aorta it sends off to the left the splenic artery $(s p . a)$, which passes to the dorsal side of the cardiac division of the stomach, supplying the latter as well as the spleen: soon after giving off the splenic artery the cœliac divides into three : of these the middle one, which continues the direction of the cœliac, is the hepatic artery (h.a); it sends branches to the lesser curvature of the stomach, and then turns forwards and sends a twig to each lobe of the liver, and a cystic artery $(c y . a)$ to the gall bladder : coming ( $\mathrm{ff}$ to the right of the hepatic is the duodenal artery $(d u . a)$, which supplies the pylorus and the proximal part of the duodenum : coming off to the left of the hepatic is the gastric artery $(g . a)$, by which the main part of the stomach is supplied.

I99. The portal vein $\left(\not \cdot \tau^{\prime}\right)$, a large, thin-walled vessel, usually gorged with blood, situated dorsal to (beneath in the present position of the parts) the artery and duct. Anteriorly it passes into and breaks up in the liver, sending a branch to each of the lobes; posteriorly it is constituted by the mesenteric vein ( $\$ \mathrm{I} 93, m . v)$ towards the animal's right side, and the lieno-gastric vein $(l . g . v)$, which brings the blood from the stomach and spleen towards the left.

200. The ramifications of the branches of the cœliac 
and mesenteric arteries, and of the tributaries of the portal vein in the mesentery and on the walls of the intestine: in nearly every case an artery and a vein run side by side, the vein being easily distinguishable from the artery by its greater diameter and thinner walls, and by retaining its blood after death.

20r. The lacteals, delicate, transparent, colourless vessels in the mesentery, running more or less parallel to the arteries and veins.

XXI. Pass double ligatures round the rectum just anterior to the pelvic cavity, and round the portal vein just before it enters the liver (the hepatic artery and bile-duct may be included in the last ligature). Cut the rectum and vein between the ligatures, cut through the mesenteric attachments of the stomach and intestines, and remove them from the body. Unravel the intestine by cutting or tearing the mesentery, and make out :

202. The relations of the various divisions of the intestine : the duodenum passes insensibly into the ileum : the ileum at its posterior end enters an ovoidal sac with glandular walls, the sacculus rotundus, which marks the junction between ileum, colon, and cæcum : the cæcum preserves a tolerably uniform diameter for the greater part of its length, finally narrowing suddenly to form the appendix vermiformis : the first portion of the colon passes insensibly into the cæcum, of which it appears to form the proximal part; about one to two inches from the sacculus rotundus it suddenly narrows and takes on its characteristic sacculations, at the same time making a sharp bend so as to run almost parallel to its former course : the transition between the colon and rectum is also very gradual, the sacculations of the former becoming fainter and fainter. 
203. The length of the intestine as a whole: it is usually about 15 or 16 times that of the animal to which it belongs. The lengths of its five divisions, duodenum, ileum, cæcum, colon, and rectum.

204. The sacculations of the colon occur in three longitudinal rows, separated by three muscular bands, or tæniæ coli, which pass, at equal distances from one another, along the whole length of the colon.

205. The Peyer's patches, masses of lymphoid tissue occurring in the form of thickener areas on the walls of the intestine : those on the ileum are oval and abuut half an inch long : on the wide portion of the colon, close to the sacculus rotundus is a larger patch about three-quarters of an inch in diameter: the sacculus rotundus itself and the appendix vermiformis are in reality large Peyer's patches.

XXII. Cut open the stomach, and parts of the small intestine, colon, rectum, cæcum, and appendix vermiformis : in another specimen remove the cæcum with a small portion of the ileum and colon, ciear out its contents by directing a stream of water through it, and either distend with air and dry, or, better, distend with a $0^{\circ} 5$ per cent solution of chromic acid and place in a vessel of the same fluid until hardened : in either case cut apertures in various parts so as to see the interior. Note the following points in the internal structure of the alimentary canal :

206. The three coats of the stomach : the outer peritoneal investment, the middle muscular layer, and the inner layer of mucous membrane : the great thickness of the muscular layer in the antrum pylori : the slightly different character of the mucous membrane in the cardiac and pyloric regions : the irregularly longitudinal rugæ into which the mucous membrine is raised.

207. The thick projecting rim, or pyloric valve between the stomach and duodenum.

208. The prominent aperture of the bile-duct in the duodenum just beyond the pylorus.

209. The three coats of the intestines : much thinner, for the most part, than those of the stomach.

210. The irregular transverse ridges or valvulæ conniventes of the mucous membrane of the small intestine : the minute conical projections or villi which beset these: the latter can only be seen to advantage by examining under water. 
$2 \mathrm{II}$. The rounded aperture between the sacculus rotundus and the colon, its margins forming the ileo-colic valve.

212. The spiral valve of the cacum, a narrow spiral hand projecting into the cavity of the blind gut and corresponding with the external marling.

213. The intra-colic valve, a prominent fold occurring at the bend between the two portions of the colon.

214. The thick glandular walls of the appendix vermiformis and sacculus rotundus: the very thin walls of the crcum itself, and the minute papillary elevations with which its inner surface and that of the spiral valve are beset.

215. The absence of villi in the large intestine: the papillary surface of the mucous membrane of the colon, the smooth character of that of the rectum.

XXIII. Tie the postcaval vein at the points where it enters and leaves the liver: remove the whole of the liver, with the exception of the part which surrounds the postcaval. Note:

216. The transparent, shining central tendon of the diaphragm, and the muscular fibres which radiate from it to the body walls, and are collected dorsally into two strong bands of muscle, the pillars of the diaphragm, which pass backwards to their origin from the anterior lumbar vertebræ.

217. The aorta, passing from the abdominal cavity forwards between the pillars of the diaphragm.

218 . The œsophagus, passing through an aperture in the dorsal region of the central tendon.

219. The postcaval, passing through an aperture situated to the right and ventral of that for the gullet.

220. The three hepatic veins (Fig. $61, h . v)$, by which the blood is taken from the liver to the postcaval : they are best seen by slitting up the intra-hepatic part of that vein: iwo of them enter the postcaval immediately posterior to 
the diaphragm, the third near the point at which the postcaval enters the liver.

22I. The posterior phrenic veins $(i . p h)$ taking the blood from the abdominal surface of the diaphragm to the postcaval.

XXIV. Cut away enough of the ribs on one or both sides to get a good view of the interior of the thorax, and make out :

222. The relations of the pleuræ: the outer or parietal layer of each lines its own half of the thorax, to the walls of which it adheres closely ( $\$$ I6I): the adjacent or inner faces of the two parietal layers form a double median vertical partition, the mediastinum: the mediastinal space included between the two layers of this partition is largely occupied by the heart and its surrounding pericardium, and is thus practically obliterated in the greater part of its extent: it is, however, readily made out in the interval between the heart and the sternum, where it is called the ventral (anterior) mediastinal space : at the entrance of the bronchi each pleura is reflected over the corresponding lung, forming the thin, closely adherent viscerail layer.

$\mathrm{XXV}$. Cut through the posterior end of the sternum: separate the mediastinum from the dorsal surface of the bone and turn it forwards: open the pericardium by a longitudinal incision, and make out :

223. The relations of the pericardium: its outer or parietal layer forms a loose bag investing the heart, and, at the origins of the great vessels ( $\$ 229-232$ and 237), reflected on to the surface of the heart, forming the thin, closely adherent visceral layer: in the space included between the two layers is contained a small quantity of colourless pericardial fluid. 
XXVI. Dissect away the pericardium, the thymus gland, and any fat about the base of the heart which may obscure the vessels coming from it. Follow out these vessels to the head and anterior extremities by clearing away the muscle, connective tissue, fat, \&c., by which they are surrounded. Also clean the aorta $(\$ 23 i)$ and postcaval, and follow out their branches into the posterior extremities, taking care not to injure the ureters ( $\$ 262$ ), and the vasa deferentia $(\$ 267)$ or uteri ( $\$ 288)$. Make out :

224. The left ventricle (Fig. GI, l. $\tau^{\prime}$ ), which forms the whole apex of the heart: it is lighter in colour than the rest of the organ and harder to the touch.

225. The right ventricle $\left(r . v^{\prime}\right)$, divided by an oblique depression from the left ventricle: it is darker in colour than the left and softer to the touch.

226. The right and left auricles (r.au, l.au), situated above (anterior to) the corresponding ventricles : they are small, dark, thin-walled chambers, slightly overlapping the bases of the ventricles.

227. The ramifications of the coronary artery and vein over the surface of the ventricles.

228. 'The bifurcation of the trachea, at about the level of the base of the heart, to form the two bronchi, one of which passes to each lung.

229. The pulmonary artery $(p . a)$, proceeding from the cone-like anterior prolongation of the right ventricle, and passing forwards and to the left : it soon divides into a right and a left trunk, one of which passes to each lung.

230. The puimonary veins $\left(p \cdot v^{\prime}\right)$, returning the blood from the lung to the left auricle. They are best seen at a later stage (see $\$ 306$ ). 


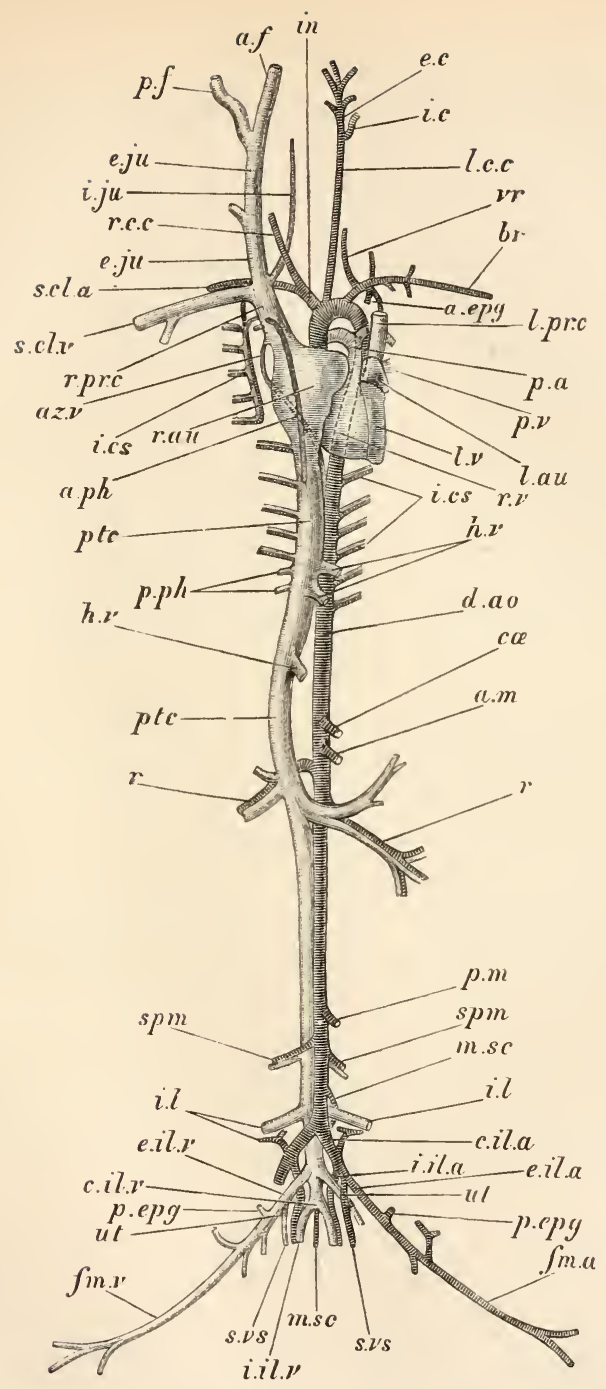

FIG. 6I.-Lepus cuniculus. The vascular system $\left(\times \frac{1}{2}\right)$. 
The apex of the heart is somewhat displaced towards the left of the subject : the arteries of the right and the veins of the left side are in great measure remuved.

a. ao, arch of the aorta : a.eps, internal mammary artery : a.f, anterior facial vein : $a . m$, anterior mesenteric artery : $a . p h$, anterior phrenic vein : $a z . v$, azygos vein : br, brachial artery : c.il.a, common iliac artery: c.il.v, common iliac vein: $c \alpha$, cœlıac artery: d.ao, dorsal aora: e.c, external carotid artery : e.il.ı, external iliac artery : e.il.z, external iliac vein : e.ju, external jugular vein : $j m . a, f(m o r a l$ artery : fm.v, temoral vein : h.v, hepatic veins : i.c, internal carotid artery : i.cs, intercontal vesiels: i.il.a, internal iliac artery : i.il.v, internal iliac vein $: i . j u$, internal jugular vein $: i . l$, ilio-lumbar artery and vein $: i n$, innominate artery : l.au, left atricle : l.r.c, left common carotid artery : l.prc, left precaval vein : $l . v$, left ventricle $: m . s c$, median sacral artery : $p . \alpha$, pulmonary artery : $p . e p g$, epignstric artery and vein $: p . f$, posterior facial vein $: \hat{p} \cdot m$, posterior mesenteric artery $: p \cdot p h$, posterior phrenic veins : $p t c$, postcaval vein $: p . v$, pulmonary vein $: r$, renal artery and vein : r.au, right auricle : r.c.c, right common carotid artery: r.prc, right precaval vein : $r . v$, right ventricle: $s c l . a$, subclavian artery : scl. $z^{\prime}$, subclavian vein : spm, spermatic artery and vein : s. $z / s$, superior vesical artery and vein : $u t$, uterine artery and vein : $v r$, vertebral artery.

231. The two precaval veins, or venæ cavæ superiores (r.pre, l.prc), passing from the anterior end of the thorax towards the base of the heart: each is formed by the union of the subclavian vein $(s c l . v)$, from the corresponding arm, and the external jugular vein (c.ju) already noticed ( $\$ \mathrm{I} z 7)$ from the head ; the right precaval is seen at once to enter the right auricle: the left trunk is scen, on lifting up the heart, to curve round the dorsal side of the base of that organ, and enter the same chamber. Sometimes the two external jugular veins unite with one another by a transverse connecting trunk in the posterior region of the neck.

The external jugular is constituted anteriorly by the anterior facial vein (a.f), which runs just within the ramus of the mandible, and the posterior facial vein, $(p . f)$, coming from the external ear ; near its junction with the subclavian, it receives the internal jugular vein $(i . j u)$, which runs close alongside the trachea, and receives the blood from the brain. 
232. The thoracic portion of the postcaval vein $(p t c)$, passing forwards from the diaphragm to the right auricle.

233. The anterior phrenic vein $(a . p h)$, passing forwards from the diaphragm, alongside the postcaval, and pouring its contents into the right precaval.

234. The œsophagus, or gullet, passing from the anterior boundary of the thorax, dorsal to the trachea and between the lungs, backwards to its aperture in the diaphragm.

235. The paired phrenic nerves, passing from the anterior boundary of the thorax backwards to the diaphragm, which they supply: the right nerve runs alongside the right precaval, across the right auricle and along the postcaval, the left nerve runs along the left precaval and then passes just internal to the left lung.

Traced forwards, the phrenic is found to take its origin from the sixth cervical nerve : if followed beyond the thorax, it should be left until the nerves of the neck (\$\$ 244-246) are dissected.

236. The thoracic portions of the vagi (see $\$ 186$ and 244 ): the right vagus enters the thorax to the right of the trachea along which it runs, thence passing on to the gullet and so through the diaphragm to the stomach : the left nerve runs at first parallel to the left precaval, finally also reaching the gullet, along which both nerves take a spiral course.

237. The aorta (ao), a large, elastic, thick-walled vessel, of a whitish colour, proceeding from the base of the heart behind the pulmonary artery; it rises clear of the heart, then bends over to the left side, forming the arch of the aorta (a.av), and passes backwards, arching over the left bronchus $(\S 228)$ to reach the ventral face of the spinal column, along which it passes as the dorsal aorta (d.ao) throughout the whole length of the thorax and abdomen, dividing at last into the two common iliac arteries 
(c.il.a, § 258) which pass backwards and outwards to the hind legs. The aorta is united to the pulmonary artery by a short ligament, the remains of the embryonic ductus arteriosus.

23S. The thoracic duct, or main trunk of the lymphatic system : a slender, thin-walled tube, lying to the dorsal side of the aorta, and pouring its contents into the left subclavian vein at its junction with the left external jugular : it is best seen by pulling the thoracic aorta somewhat to the right.

239. The thoracic portion of the sympathetic nerves, slender white cords, lying one on each side of the aorta, and having at intervals ganglia, which lie on the heads of the ribs; each is continued backwards into the abdomen ( $\left(\right.$ I $_{5}$ ) and forwards into the head ( $\$ 245$ ).

240. The azygos or (right) cardinal vein (az.v), best seen by turning the heart and lungs over to the left side : it lies immediately to the right of the thoracic aorta, receiving the intercostal veins (i.cs) from the spaces between the seven posterior ribs: anteriorly it pours its contents into the right precaval. The blood from the five anterior intercostal spaces is returned, on each side, into an anterior intercostal vein which enters the corresponding precaval.

24I. The innominate artery $(i n)$, springing from the arch of the aorta towards its right side; it gives off at its origin the left common carotid artery (l.c.c) and then, passing forward, divides into the right common carotid (r.c.c) and right subclavian (s.cl.a).

242. The left subclavian artery, taking its origin from the left side of the arch of the aorta.

243. The course of the common carotid arteries : each passes up the side of the neck, external to the trachea and internal to the external jugular, gives off branches to 
the larynx, and, at about the level of the anterior end of the larynx, divides into the internal carotid (i.c), which passes dorsalwards to reach the brain, and the external carotid $(e . c)$, which supplies the greater part of the head: the internal carotid may be recognised as being the first important branch given off from the outer side of the common carotid: the external carotid divides almost inmediately into branches for the jaws, eye, \&c.

244. The cervical portion of the vagus, a nerve rurning to the outer side of each common carotid.

Traced backwards, the right vagus is seen to pass across (ventral to) the right subclavian artery, the left across the arch of the aorta, to enter the thorax, where they have already been seen ( $\$ 236$ ).

245. The cervical portion of the sympathetic, seen by lifting up the carotid and carefully separating it from the vagus : it is a flat pale nerve lying immediately dorsal to the artery : traced backwards, it enlarges at about the level of the first rib into the posterior cervical ganglion, and thus enters the thorax, where it has already been seen.

246. Traced forward;, the sympathetic is seen to enlarge, at a level just anterior to the larynx, into the anterior cervical ganglion, immediately external to which is a similar enlargement of the vagus, the ganglion of the root: crossing both nerves and the carotid artery at about the same level, but obliquely from behind forwards and inwards, is the slender tendon of the digastric muscle, and immediately behind and parallel to this the hypoglossal nerve.

247. The thyroid gland, a brownish mass, consisting of two lobes applied to the sides of the larynx, and connected across the ventral face of the latter by a band-like intermediate portion.

248. The large thyroid cartilage, forming the anterior part of the larynx, and the ring-like cricoid cartilage, forming its posterior part.

249. The submaxillary gland has been already seen: its duct (Wharton's duct) is a very fine tube proceeding from its inner face to open on the floor of the mouth. 
250. 'The course of the subclavian arteries: each, soon after its origin, divides into the vertebral artery $(v r)$, which dives inwards, and then passes forwards through the vertebrarterial canal of the cervical vertebræ ( $\S 24)$, to supply the brain and spinal cord ; and the brachial artery (br), which passes directly outwards, and sends branches to the fore-limb and shoulder-girdle, giving off also the internal mammary artery (a.epg), a small vessel which runs backwards over the inner face of the wall of the thorax just external to the sternum.

25I. The thoracic arteries, small vessels given off in pairs from the thoracic aorta, and supplying the seven posterior intercostal spaces. The five anterior intercostal spaces are supplied by a branch of the internal mammary.

252. The phrenic arteries, arising from the aorta and supplying the diaphragm.

253. The renal arteries $(r)$, of which the right comes off almost immediately posterior to the anterior mesenteric ( $\left(\mathrm{I} 8_{3}\right)$, and the left about half an inch posterior to the right; each passes immediately to the hilus $(\$ 26 \mathrm{r})$ of the corresponding kidney.

254. The renal veins $(r)$, each of which runs parallel and just posterior to the corresponding renal artery, receives a large vein from the dorsal wall of the abdomen, and pours its contents into the postcaval.

255. The lumbar arteries and veins, small vessels supplying the dorsal walls of the abdomen, and springing from the aorta and postcaval respectively.

256 . The spermatic arteries and veins $(s p m)$, taking their origin somewhat posteriorly to the posterior mesenteric artery, from the aorta and postcaval respectively, and passing to the testes ( $\$ 266$ and 268 ), or ovaries ( $\$ 29 \mathrm{r}$ ).

257. The median sacral artery ( $\left.m . s^{i}\right)$, a small trunk arising from 
the dorsal side of the àrta, and passing backwards to the pelvis : it corresponds to the caudal continuation of the aorta (see pp. $67,1_{5}$, 235).

258. The common iliac arteries (c.il.a), formed by the bifurcation of the aorta: each gives off soon after its origin the ilio-lumbar artery (i.l) to the posterior part of the dorsal abdomiral walls, and then almost immediately divides into the internal iliac (i.il.a), which passes along the dorsal wall of the pelvic cavity, and the external iliac (e.il.a), which gives off an artery to the bladder and uterus, and then, passing beneath Poupart's ligament, becomes the femoral artery $(f m . a)$, and supplies the leg. Immediately external to Poupart's ligament the femoral gives off the posterior epigastric artery $(p . e p g)$, which is distributed to the ventral abdominal walls.

259. The ilio-lumbar veins (i.l), large vessels which enter the postcaval just anterior to the bifurcation of the aorta.

In some cases the left ilio-lumbar instead of directly entering the postcaval, turns forwards, runs parallel to the latter, receives the left spermatic, and enters the postcaval a little posterior to the entrance of the left renal.

260. The trifurcation of the inferior cava at its posterior end into the two outwardly directed external iliac veins (e.il.i), and the median, backwardly directed common iliac vein (c.il.v). The external iliacs, like the corresponding: arteries to which they are parallel, are the intra-abdominal portions of the femoral veins $(f m . v)$, by which the blood is returned from the legs; they also receive the blood from the bladder and uterus: the posterior epigastric veins $(p \cdot e p g)$, open into the femorals immediately external to Poupart's ligament. The common iliac is formed by the union of the two internal iliac veins (i.il.v), which run 
parallel and internal to the corresponding arteries along the dorsal wall of the pelvis.

XXVII. Carefully dissect away the peritoneum from the kidneys, ureters, and genital organs, already noticed, as well as the fat which usually obscures them. Make out :

26I. The kidneys, compact red bodies of characteristic shape, lying one on either side of the lumbar region, the right considerably in front of the left. Each has on its inner edge a depression or hilus, and is covered by peritoneum on its ventral side only.

262. The ureter (Fig. 62, A and B $u r$ ) a long white tube springing from the hilus, about one-eighth of an inch in diameter in its upper part, but rapidly getting narrower: the two ureters pass somewhat inwards towards the middle line, and then backwards almost parallel with one another, and enter

263 . The bladder ( $b l)$, a pyriform sac, which may vary greatly in size and appearance, according to its state of distension; when full, it projects some distance into the abdominal cavity, and its walls appear very thin and show the bright yellow urine through them; when empty it hardly appears above the pubes, and its walls appear thick and opaque: it may be inflated from the urethra (\$ $\mathrm{I}_{53}$ ). The ureters enter it about half way (in the collapsed condition) between its broad anterior end, or fundus, and its narrow neck.

264. The adrenals, or suprarenal bodies, small, yellowish-white bodies, of which the right is close to the corresponding kidney, while the left lies in the anterior angle between the aorta and renal artery.

In the Maie.

265. The penis (A, c.c, c.s, g.p), projecting backwards 
from the posterior edge of the symphysis pubis, and enclosed in a loose fold of skin, the prepuce : it is strengthened
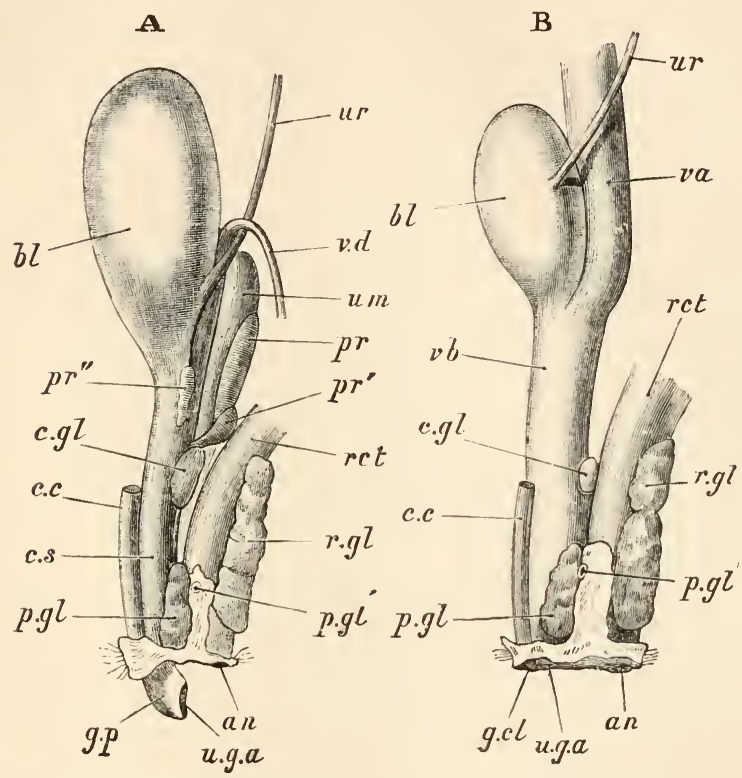

FIG. 62.-Lepus cuniculus. The urinogenital organs, A, of the male, $\dot{B}$, of the female, from the left side ( $\frac{1}{2}$ nat. size).

The kidneys and proximal ends of the ureters, and in B the ovaries, Fallopian tubes, and uteri, are not shown.

$a n$, anus : $b l$, urinary bladder: c.c, corpus cavernosum : c.s, corpus spongiosum: $c . g l$, Cowper's gland : $g . c l$, glans clitoridis: $g \cdot p$, glans penis : $p \cdot g l$, perineal gland $: \not p \cdot g l^{\prime}$, aperture of its duct on the perineal space : $p r$, anterior, $p r^{\prime}$, posterior, and $p r^{\prime \prime}$, lateral, lobes of prostate : $r c t$, rectum : $r . g l$, rectal gland: $u . g . a$, urinogenital aperture: $u . m$, uterus masculinus: $u r$, ureter : $v a$, vagina : $v b$, vestibule : $v . d$, vas deferens.

ventrally by the two hard, closely applied corpora cavernosa (c.c), which, at the proximal end of the organ, 
diverge, forming the crura penis, and are attached to the ischia : dorsally it is soft and yielding, consisting only of the thin vascular corpus spongiosum (c.s, see $\$ 279)$ : its free end is formed by a soft conical body, the glans penis $(g . p)$, which projects beyond the prepuce, and bears the slitlike aperture $(u . g . a)$ of the urethra, a canal traversing the penis.

266. The scrotal sacs, situaterl one on each side of the penis, and having their cavities in communication, by a widish aperture, with the peritoneal cavity, so that by pulling upon the spermatic artery and vein $\left(\$ 25^{6}\right)$ the testis which each contains can be retracted into the abdomen.

267. The vasa deferentia (v. $a)$, two whitish tubes resembling the ureters in appearance and size: they proceed from the scrotal sacs into the peritoneal cavity, and then each curves over the corresponding ureter to reach the dursal side of the bladder.

XXVIII. Slit open one of the scrotal sacs along its ventral wall, and make out:

268. The testis, a pinkish-white, ovoidal body, about an inch long in a full-grown rabbit

269. The epididymis, an irregular, convoluted body, forming the greatly coiled proximal end of the vas deferens : it consists of the caput epididymis, applied to the anterior end of the testis, and connected by a narrow band running along the inner edge of the latter, with the cauda epididymis, which is continued posteriorly beyond the testis, and, anteriorly, gives origin to the vas deferens. The caput is usually much obscured by a mass of fat, in which run the spermatic artery and vein.

270. The gubernaculum, a short cord connecting the posterior end of the cauda epididymis with the interior of the scrotal sac. 
27I. The coats of the scrotal sac: these are, a layer of skin externally; then a layer of loose connective tissue, the dartos; then a layer of muscle, the cremaster, continuous with the internal oblique muscle of the abdomen ; and, lastly, an internal lining of peritoneum, the tunica vaginalis.

272. The mesorchium, a double fold of peritoneum suspending the testis and vas deferens to the dorsal wall of the scrotal sac, after the manner of a mesentery : its two layers are continuous with the peritoneal lining of the sac (tunica vaginalis), and are reflected over the testis.

XXIX. Dissect away the kidneys, ureters, and scrotal sacs from the surrounding parts: cut away the symphysis pubis with bone forceps: cut through the two corpora cavernosa $\left(\$_{2} 6_{5}\right)$ by which the penis is attached to the ischia. Remove the whole of the urinogenital organs from the body, together with the posterior end of the rectum, and pin them out in a dissecting dish, with the dorsal surface uppermost, taking care to preserve the natural relation of the parts. Carefully dissect away all fat and connective tissue, gradually separate the rectum from the other organs and turn it aside, making out :

273. The uterus masculinus $(u . m)$, a large sac, slightly bilobed at its anterior end, and lying against the dorsal side of the neck of the bladder. The vasa deferentia, after curving round the ureters, are seen to pass between this sac and the bladder.

274. The prostate $(p r)$, a glandular mass, in relation with the neck of the bladder and the uterus masculinus.

It consists of five lobes : an anterior $(p r)$, so closely applied to the dorsal wall of the uterus masculinus as to be almost inseparable from it: another posterior $\left(p r^{\prime}\right)$, more prominent than the first, bilobed anteriorly, and easily separable from the uterus nuasculinus; and two 
small lateral lobes $\left(p r^{\prime \prime}\right)$, lying along the sides of the neck of the bladder.

275. Cowper's glands $(c . g l)$, a pair of brown, ovoid masses, immediately behind the posterior lobe of the prostate, and enclosed in a layer of muscle.

$2 ; 6$. The rectal glands $(r . g l)$, two brown elongated glands, about an inch long and a quarter of an inch wide, situated one on either side of the rectum.

277 . The perineal glands $(p . g l)$, similar in appearance to the rectal glands, but not more than half an inch long: they lie, one on either side of the extremity of the penis, and are in relation externally one with each of the hairless perineal spaces, on the surface of which its duct opens on a small papilla $\left(p \cdot g l^{\prime}\right)$.

278 . By slitting open the uterus masculinus along its dorsal wall, the vasa deferentia are seen to open into its ventral wall, near its junction with the bladder.

279. The corpus spongiosum (c.s), a quantity of extremely vascular tissue surrounding the urethra, from about the posterior boundary of Cowper's glands to the glans penis, with which it becomes continuous: to see it satisfactorily, the rectum and rectal glands should be entirely removed.

XXX. Turn the organs with the ventral side uppermost, and make a median incision along the whole length of the penis, beginning at the glans and cutting through the fibrous septum between the corpora cavernosa: continue the incision forward so as to open the bladder along its ventral wall. Note :

280. The small apertures of the ureters in the dorsal wall of the bladder at about the junction of its middle and posterior thirds. 
28I. The verumontanum or colliculus seminalis, a small rounded elevation on the dorsal wall of the urethra, just posterior to its junction with the bladder.

282. The opening of the uterus masculinus into the urethra: a crescentic aperture just in front of the anterior edge of the verumontanum.

283. The openings of the prostatic ducts, minute apertures, usually about four in number, on each side of the verumontanum.

284. The openings of the ducts of Cowper's glands, four extremely minute apertures in the dorsal wall of the urethra, about half an inch posterior to the verumontanum.

\section{In the Female.}

285. The vulva, or external urinogenital aperture, (Fig. 62, B, u.g.a) guarded by two tolerably prominent folds of mucous membrane, the labia majora, and leading into a wide tube, the vestibule, situated immediately ventral to the extremity of the rectum.

286. The clitoris $(c . c, g . c l)$, a hard, rod-like body embedded in the ventral wall of the vestibule: it consists of two closely applied corpora cavernosa (c.c), resembling the bodies of the same name in the male ( $\$ 265)$, and, like them, diverging anteriorly as the crura clitoridis to be attached to the ischia: the free end of the clitoris is formed by the glans clitoridis $(g . c l)$, a small soft conical body between the anterior (ventral) ends of the labia majora.

287. The vagina (Figs. 62, B, and $63, \tau a$ ), a widish tube lying between the bladder and the rectum; like the other reproductive organs, it varies greatly in size according to the age of the animal.

288. The uteri (Fig. $6_{3}, r . u t$, l.ut), paired tubes proceeding from the anterior end of the vagina, and proceeding outwards towards the flanks; they are comparatively wide 
at their posterior or vaginal ends, but become narrow anteriorly and pass almost insensibly into

289. The Faliopian tubes ( $f$ l.t), or anterior extremities of the genital ducts: they are somewhat convoluted tubes of small calibre, enaing anteriorly in a wide, membranous, funnel-shaped extremity with fimbriated margin.

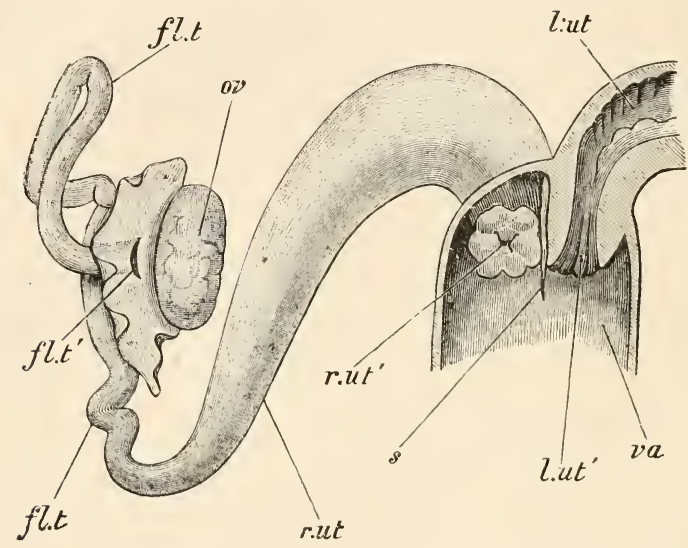

Fig. 63.-Lepus cuniculus. The anterior end of the vagina, with the right uterus, Fallopian tube, and ovary (nat. size).

Part of the ventral wall of the vagina is removed, and the proximal end of the left uterus is shown in longitudinal section.

f.t, Fallopian tube : $f$. $t^{\prime}$, its peritoneal aperture: l.ut, left uterus : l.ut', left os uteri : r.ut, right uterus : $r . u t^{\prime}$, right os uteri $: s$, vaginal septum : $v a$, vagina.

290. The broad ligament, a sheet of peritoneum, suspending the uterus and Fallopian tube to the dorsal body wall.

29r. The ovaries (ov), ovoidal bodies, about half an inch long and a quarter wide, situated just external to the fimbriated extremity of the Fallopian tube: they have a whitish colour, and are studded over with small round 
pinkish projections, the Graafian follicles, each of which contains an ovum.

XXXI. Cut through the symphysis pubis, and dissect away the urinogenital organs from the body, fasten them with the dorsal side upwards, and make out the rectal and perineal glands, which have the same relation as in the male (see $\$ 276$ and 277 ); then remove these glands along with the rectum, and make out

292. The vestibule (Fig. 62, B, $\tau^{\prime} \dot{b}$ ), a wide tube, with the dorsal wall of which the rectum was in contact: with its anterior end the bladder and vagina are connected, while, posteriorly, it opens externally by the vulva. It has precisely the relations of the urethra of the male $\left(\$ 26_{5}\right)$, and its walls are invested with a vascular tissue answering to the corpus spongiosum ( $\$ 279)$.

293. Cowper's glands (c.gl), a pair of small whitish masses, lying on the dorsal wall of the vestibule at about the middle of its length.

XXXII. Turn the organs with their ventral side upwards, make a median longitudinal incision through the vestibule, and continue it forwards until the cavity of the bladder is exposed: make a median longitudinal incision through the ventral wall of the vagina: afterwards slit up one of the uteri and the corresponding Fallopian tube along their whole length. Make out :

294. The small aperture of the ureters in the dorsal wall of the bladder about half way between its fundus and its neck.

295. The almost insensible passage from the bladder to the vestibule, the neck of the former being very wide.

296. The irregular rugæ or ridges of mucous membrane into which the walls of the vestibule are raised. 
297. The large, somewhat crescentic aperture by which the vagina opens into the vestibule.

298. The small apertures of the ducts of Cowper's glands, on the dorsa! wall of the vestibule.

299. The os uteri (Fig. 63, r.ut'), a small aperture with a greatly thickened and rugose margin, on each side of the anterior end of the vagina, and leading into the corresponding uterus. Between the two ora uterorum is a rudimentary vertical septum $(s)$ tending to divide the vagina into two tubes.

300. 'The very thick walls of the uterus, consisting of an outer muscular layer and an inner mucous layer raised up into very large and prominent rugæ.

301. The smooth walls of the Fallopian tube and the opening of that tube posteriorly into the uterus, and anteriorly by its funnel-like fimbriated extremity ( $\left.f . t^{\prime}\right)$ into the peritoneal cavity in the immediate neighbourhood of the ovary.

In both sexes.

XXXIII. Make a section of one of the kidneys, through the hilus, and parallel to the dorsal and ventral faces of the organ. Note:

302. The sinus, a large cavity excavated in the substance of the kidney into which the hilus leads.

303. The pelvis, a large funnel-like dilatation of the proximal end of the ureter, nearly filling the sinus: it ends in a number of small subdivisions, the calices.

304. The urinary pyramid, a conical mass of kidney substance, projecting into the pelvis.

305. The distinction between the superficial or cortical portion of the kidney which has a dotted appearance, and its central or medullary portion, marked with striæ which radiate outwards from the pyramid. 
XXXIV. Carefully remove from the body the heart and lungs, together with the posterior end of the trachea and recognisable portions of the aorta and venæ cavæ. Fasten out the organs under water, with their dorsal surface uppermost, and make out:

306. The course of the pulmonary arteries and veins: the arteries remain single until they reach the lungs; the veins are two from each lung, and enter the left auricle separately, in the $\mathrm{U}$-shaped space between the two precavals.

XXXV. Cut through the pulmonary arteries and veins close to the lungs, and separate the latter from the heart : make out :

307. The division of the left lung into two lobes, an anterior and a posterior: the former is deeply divided by a transverse fissure.

308. The division of the right lung into four lobes, called, passing from before backwards, the anterior accessory, the anterior, the posterior, and the posterior accessory: the "accessory" lobes are considerably smaller than the other two: the posterior accessory is situated internal to the root of the lung and rather to the left side of the thoracic cavity.

309. The cartilages of the trachea and bronchi, incomplete rings surrounding the ventral and lateral aspects of the tubes, but leaving their dorsal sides unstrengthened. lobe.

310. The division of the left bronchus into two tubes, one for each

3II. The subdivisions of the right bronchus: it first gives off a branch on the outer side to the anterior accessory lobe, then one on the ventral side to the anterior lobe, and finally divides into two branches for the posterior and posterior accessory lobes. In some instances the 
bronchus for the anterior accessory lobe is given off from the trachea immediately before its bifurcation.

3I2. By scraping away the lung substance the bronchi may be further followed: their cartilages soon lose the incomplete annular form, and become irregular isolated patches: the branches given off from the bronchi into the substance of the lung come off at an acute angle.

XXXVI. In the heart cut away the outer walls of both auricles, so as to expose their cavities, taking care not to injure the venæ cavæ or pulmonary veins. Note :

313. The division of each auricle into a larger, smoothwalled, posterior portion or atrium, and a small, anterior portion or appendix auriculæ, which projects over the base of the ventricle, and the walls of which are strengthened internally by a network of muscular bands, the musculi pectinati.

314. The septum auricularum, or muscular division wall between the auricles.

315. The fossa ovalis, an oval area on the septum rather thinner than the rest of it; it is seen best from the left side by holding the septum up to the light, and is surrounded by a slightly raised margin, the annulus ovalis. It marks the position of the foramen ovale of the foetus.

316. The auriculo-ventricular apertures, by which the auricles respectively communicate with the ventricles.

317. The apertures of the pre- and postcaval veins into right auricle: that of the right precaval is in the anterior (upper) end of the auricle; that of the postcaval in the postero-dorsal region, a membranous fold, the remains of the fotal Eustachian valve, extending from its posterior margin towards the septum; that of the left precaval is immediately to the left of, and posterior to, the Eustachian valve, and is bounded behind (below in 
the upright position of the heart) by a semi-lunar fold, the valve of Thebesius.

318. The aperture of the coronary vein, by which the blood is brought back from the substance of the heart; it is a small round opening just within the margin of the tunnel-like aperture of the left precaval.

319. The apertures of the pulmonary veins into the left auricle.

XXXVII. Cut away both auricles so as to expose the bases of the ventricles: cut away all but about an eighth of an inch of the aorta and pulmonary artery : pour water into the ventricles through the auriculo-ventricular apertures. Note:

320. The auriculo-ventricular valves, two sets of membranous flaps, which, when the filled ventricles are squeezed, come together at their edges and close the auriculo-ventricular apertures; but when the pressure is released fall down into the ventricles. On the left side are two flaps, together constituting the mitral or bicuspid valve; on the right side are three flaps, together constituting the tricuspid valve.

32r. The semilunar valves, of which there are three at the origin of both the pulmonary artery and aorta: all three valves, in each case, are in one plane, and when there is fluid in the artery, come together by their edges, and close the passage to the ventricle; but when the ventricle is squeezed, the pressure forces them aside, and causes them to flap back against the walls of the artery.

322. The sinuses of Valsalva, slight dilatations of the aorta and pulmonary artery, just anterior to (above) the semilunar valves.

323. The apertures of the coronary arteries, by which 
the heart is supplied with blood, situated in two of the aortic sinuses of Valsalva.

XXXVIII. Remove the outer walls of both ventricles, by making first a transverse incision along the base of each, and then taking from its extremities converging incisions nearly to the apex of the heart. Make out :

324. The comparatively thin walls of the right ventricle, and the extremely thick walls of the left ventricle.

325. The septum ventriculorum, or partition between the ventricles; it is convex towards the right, concave towards the left side, so that, in transverse section, the cavity of the right ventricle appears semilunar, that of the left almost circular.

326. The columnæ carneæ, muscular ridges into which the inner surface of the ventricular walls is raised. One of those in the right ventricle usually takes on the form of a cord extending across the cavity from the inner to the outer side, and called the moderator band.

327. In the right ventricle, the three flaps of the tricuspid valve attached by their anterior edges round the auriculo-ventricular aperture: their irregular posterior edges, which depend into the ventricle, are attached by strings, the chordæ tendineæ, to small conical elevations of the ventricular wall, the musculi papillares.

328. In the left ventricle, the two flaps of the mitral valve, attached in the same manner as those of the tricuspid. The papillary muscles are, however, fewer in number and considerably larger in size.

329. The conus arteriosus, or infundibulum, a prolongation of the left anterior angle of the right ventricle, in the apex of which is the aperture of the pulmonary artery. 330. The aperture of the aorta in the left ventricle, 
situated within and dorsal to the auriculo-ventricular aperture.

XXXIX. Dissect away the skin from the side of the head, and make out:

331. The masseter, a large mass of muscle covering the posterior half of the mandible.

It arises from the lower edge of the jugal arch, and is inserted into the lower border of the mandibular ramus.

332. The muscular branches of the seventh or facial nerve, passing from behind forwards over the outer surface of the masseter.

Traced backwards, these nerves are seen to spring from a single trunk which makes its exit through the stylo-mastoid foramen ( $\$ 55$ ).

333. The parotid (salivary) gland, a soft, irregular, pinkish mass, situated just in front of and below the external ear.

Its fine duct (Stenson's duct) passes forwards from its anterior edge, along with the branches of the seventh nerve, and in front of the masseter muscle dips inwards, to open into the interior of the mouth.

334. The infraorbital (salivary) gland, a large lobulated mass lying in the antero-inferior region of the orbit, partly outwards and partly within the cavity.

Its duct passes downwards from its inferior edge, to open into the cavity of the mouth.

XL. ${ }^{1}$ Cut away, with bone-forceps, the supraorbital process of the frontal ( $\$ 47)$, working from behind forwards, and making out:

335. The superior oblique muscle of the eye, arising

1 The following sections (\$ 335-353) cannot conveniently be worked out in a specimen from which the brain has been removed, unless that operation has been conducted with very great care. 
from the postero-internal region of the orbit along with the recti $\left(\$ 33^{8}\right)$ and passing forwards and upwards to the under surface of the anterior end of the supraorbital process : there it passes through a tendinous loop attached to the bone, and then passing downwards and slightiy backwards is inserted into the eyeball.

336. The lacrymal gland, a pinkish mass situated in the upper and posterior region of the eyeball.

337. The Harderian gland, a white, opaque, lobulated body situated in the anterior region of the eyeball.

338. The four recti and the inferior oblique have the same relations as in the lower Vertebrata ( p. $77, \S \S 192-194$, and p. $7 \mathrm{~S}$, $\$$ § 201, 202).

339. The third, fourth, and sixth cerebral nerves, issuing from the sphenoidal fissure, and having the same general distribution to the eyemuscles as in the lower Vertebrata (pp. 77, 78, \$§ 195-197, 203).

340. The retractor bulbi, a conical muscle lying immediately within the recti, and forming a sheath round the optic nerve : it is supplied by the sixth nerve.

34I. The orbito-nasal nerve, or first division of the fifth, leaving the skull through the sphenoidal fissure and passing along the upper edge of the inner wall of the orbit.

342. The optic nerve, leaving the skull by the optic foramen, and passing to the inner and posterior region of the orbit.

XLI. ${ }^{1}$ Remove both eyes : divide one of them into an inner and an outer hemisphere by an equatorial incision, i.e. a vertical incision taken at right angles to and through the centre of a line joining the pupil and the optic nerve: divide the other into an anterior and a posterior half by a vertical incision at right angles to the first, that is passing through the pupil and entrance of the optic nerve. Note:

343. The fibrous sclerotic, the one-layered choroid,

1 As it is necessary to examine the eye while quite fresh, it will probably be found convenient in practice to substitute an ox's or sheep's eye for the rabbit's. 
the retina, the blind spot or entrance of the optic nerve, the ora serrata, the choroid processes, the lens, the iris, the pupil, and the aqueous and vitreous humours: all these have the same essential relations as in the lower Vertebrata (see p. 84, §§ 224-228).

344. The absence of a processus falciformis or pecten (p. r22, § I79, and p. 250, § 314).

345. The tapetum, a portion of the choroid which, instead of having the usual black hue, exhibits iridescent colours.

XLII. Dissect away the parotid gland : clear away the muscles, \&c., from the entire external surface of the bulla tympani $(\$ 54)$ : lay open the external auditory meatus by a longitudinal incision, until the tubular portion of the tympanic bone is reached: with bone-forceps cut away very carefully and gradually the outer wall of both tubular and bulbous portions of the tympanic bone, noting :

346. The tympanic membrane, a transparent fibrous partition, lying obliquely across the lower end of the meatus: through it can be indistinctly seen a small bone — the handle of the malleus (\$ 73)-attached to its inner surface.

347. The cartilage of the pinna, attached to the tubular portion of the tympanic bone, and with it forming the external auditory meatus, which thus consists of cartilaginous and osseous portions.

XLIII. Carefully cut away the tympanic membrane so as not to injure the manubrium mallei, and remove as much more of the tympanic bone as is necessary to display the following structures:

348. The tympanum, or tympanic cavity, bounded 
internally by the periotic, externally by the tympanic membrane, below and at the sides by the tympanic bone.

349. The auditory ossicles (see $\S \S 73-76$ ): after observing them and their muscles $(\$ \S 352,353)$ in situ, they should be removed, and examined under a low power of the microscope.

350. The fenestra ovalis and fenestra rotunda $(\$ 56)$.

351. Tne aperture of the Eustachian tube on the inner wall of the tympanum, below and anterior to the fenestra ovalis (see $\$ 55$, p. 272 , and $\$ 370$, p. 336).

352. The tensor tympani, a small muscle inserted by a fine tendon into the malleus : it arises from the alisphenoid.

353. The stapedius, a still smaller muscle, arising from the surface of the periotic, just above the fenestra rotunda, and passing forwards to be inserted into the neck of the stapes.

354. By the removal of the bulla tympani the proximal portions of the vagus and hypoglossal nerves ( $\$ 244,246)$ are exposed and may be traced to their foramina of exit from the skull $(\$ \S 43,58)$.

355. Lying immediately in front of and parallel to the hypoglossal is a much slenderer nerve, the glossopharyngeal : it makes its exit from the skull with the vagus.

356. The spinal accessory or eleventh nerve leaves the skull along with the glossopharyngeal and vagus, and passes backwards to its distribution to some of the muscles of the neck.

XLIV. Dissect off the masseter: carefully detach the ascending portion of the mandible from the pterygoid muscles which are inserted into its inner surface: with bone-forceps cut away the portion of the mandibular ramus lying posterior to the inferior dental foramen $(\S 7 \mathrm{I})$ : make out :

357. The third division of the fifth or mandibular nerve, supplying the muscles of the lower jaw and sending a branch-the gustatory nerve-to the tongue: the main trunk is continued through the inferior dental foramen to the interior of the ramus and so to the teeth. 
358. The second division of the fifth or maxillary nerve has been exposed by the removal of the eye and its muscles, and is seen to lie on the floor of the orbit : to see it satisfactorily the maxilla must be gradually broken away : it supplies the upper teeth, and a large branch passes through the infraorbital foramen $(\$ 65)$ to the snout.

XLV. Remove the remainder of the mandibular ramus of the side on which you are working: dissect away the pterygoid muscles : pass a probe from the cut end of the gullet forwards into the mouth : lay open the gullet along this, and pull the tongue downwards so as to get a good view of the interior of the mouth. Note:

359. The transversely-ridged palate (Fig. 64) or roof of the mouth, continued backwards into a soft membrane, the velum palati $(v l . p a)$.

360. The tongue $(t g)$, firmly fixed by its postero-inferior part to the floor of the mouth, presenting on its dorsal surface a number of small elevations or papillæ, and on each side of its proximal end a small, oval, ridged area, the papilla foliata.

$3^{6}$ r. The pharynx, or funnel-shaped posterior extremity of the mouth, continued backwards into the gullet.

362. The glottis, an aperture on the floor of the pharynx, leading, through the larynx, into the trachea. It is guarded in front by a large flap of cartilage, the epiglottis $(e p g)$, and behind by two small processes of mucous membrane, the cornicula laryngis $(c . l a)$.

363. The aperture of the posterior nares $(p . n a)$, an oval opening in the roof of the pharynx : a probe passed into it enters the nasal chamber (see $\S 366$ ).

364. The positions and characters of the teeth (see $\S \S 77-80$, pp. 277, 278). 


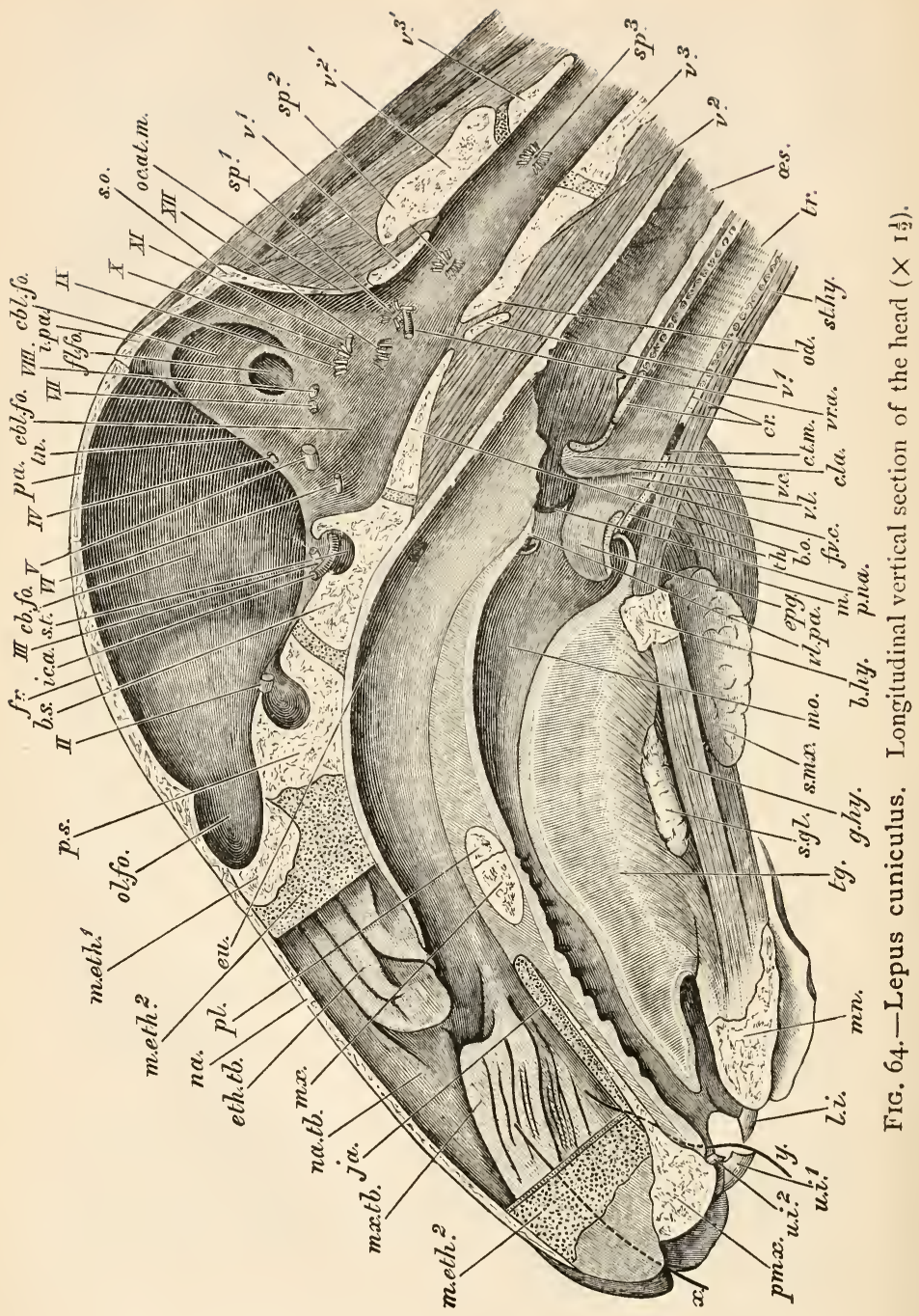


The section is taken slightly to the left of the median plane so as to clear the mesethmoid (m.eth) : all but the anterior and posterior ends of the septum nasi $\left(m . e t h^{2}\right)$ is cut away, so as to expose the right nasal cavity with its turbinals: the brain is removed, so as to show the cranial fosse and the exits of the nerves through the dura mater (it must be remembered that many of these run for a considerable distance between the dura mater and the skull before making their exit from the latter) : the muscles at the base of the tongue are partly dissected away so as to expose the right sublingual gland $(s . g l)$. The cartilage is distinguished by fine, the bone by coarse, dotting.

b.h.y', body of hyoid bone: b.o, basi-occipital : b.s, basi-sphennid: $c b$.fo, cerebral fossa : cbl.fo, cerebellar fossa : c.la, corniculum laryngis embedding arytenoid cartilage : $c r$, cricoid cartilage $: c . t . m$, crico-thyroid memhrane: epg, epiglottis: eth.ll, ethmo-turbinal: ell, aperture of Eustachian tube : $f$ l.fo, floccular fossa : fr, frontal: f.v.c, false vocal cord : g.hy, genio-hyoid muscle: i.c.a, internal carotid artery: i.pa, interparietal : $j a$, Jacobson's cartilage : $l . i$, lower incisor : $m$, masseter muscle covering edge of mandible: $m_{\text {eth }} \mathbf{i}$, lamina perpendicularis : $m . e t h^{2}$, septum nasi : $m n$, mandibular symphysis : mo, mouth cavity : $m x$, palatine plate of maxilla: $m x . t b$, maxillo-turbinal : $n \dot{a}$, nasal: $n a . t b$, naso-turbinal : oc.at.m, occipito-atlantal membrane : od, odortoid process : $a s$, œesophagus $: o l . f o$, olfactory fossa : $p a$, parietal : $p l$, palatine plate of palatine : $p m x$, premaxilla: $p . n a$, posterior nares : $p . s$, presphenoid: s.g, sublingual gland : s.mx, submaxillary gland: s.o, supra occipital: sp.I, sp.2, sp.3, roots of first three spinal nerves: st.hy', sterno-hyoid muscle: s.t, sella turcica: $t g$, tongue: th, thyroid cartilage $: t n$, tentorium $: t r$, trachea $: u \cdot i^{1}$, anterior, and $u \cdot i^{2}$, posterior, upper incisor: $v .1$, atlas; $v .2$, centrum, and $v_{.2}$, arch of axis: $v \cdot 3, v \cdot 3^{\prime}$, of third vertebra: v.c, vocal cords : $v . l$, ventricles of larynx : vi. $a$, velum palati : $v r . a$, vertebral artery : $x$, bristle passed through right nostril : $y$, bristle passed through right naso-palatine canal: $I I-X I I$, roots of cerebral nerves.

365. The small paired aperture of the naso-palatine or Stenson's canals $(y)$, situated immediately behind the posterior upper incisors $\left(u \cdot i^{2}\right)$, and leading into the nasal chamber.

XLVI. Remove the nasal, and enough of the premaxilla and maxilla to show :

366. The ethmo-, maxillo-, and naso-turbinals (eth.th, mx.tb, na.ts, $\S$ 6I, 63, 66, pp. 274, 275), and their relations to the nasal chamber in which they are contained. 367. The delicate, reddish-brown, mucous membrane (Schneiderian membrane) lining the nasal chamber and 
covering the turbinals : that on the ethmo-turbinals is distinguished as the olfactory mucous membrane.

368. The olfactory nerves, given off from the olfactory lobes to the olfactory mucous membrane: the maxiloturbinals are supplied by the maxillary nerves.

XLVII. Pass a probe from the anterior nostril into the nasal chamber as far backwards as it will go: remove the turbinals and as much of the maxilla and palatine as is necessary to show

369. The backward continuation of the nasal chamber to the posterior nares.

370. The aperture of the Eustachian tube (eui), in the dorsal wall of the posterior nasal passage, a little anterior to the posterior nares: a probe should be passed through the tube from its aperture in the tympanum ( $\$ 35 \mathrm{I})$.

37 I. The septum nasi $\left(m . e t h^{2}, \S 59\right)$, forming a median vertical partition between the anterior part of the two nasal chambers.

372. Jacobson's cartilage $(j a, \S 60)$, lying immediately external to the ventral edge of the septum nasi and ensheathed by the palatine process of the premaxilla: it has the form of a cylinder with tapering ends.

373. Jacobson's organ, seen by carefully removing the palatine process of the premaxilla and Jacobson's cartilage : it consists of a delicate tube of mucous membrane, inclosed in the scroll-like Jacobson's cartilage, and opening at its anterior end into the nasal chamber. It is supplied by branches of the olfactory nerve which pass downwards and forwards along the surface of the septum nasi.

XLVIII. Remove the larynx with the anterior part of the trachea, and dissect away the muscles, \&c., attaching them to surrounding parts. If only one larynx is to be had, make a longitudinal vertical section of it, keep one half entire, and from the other dissect away the muscles and mucous membrane so as to see the cartilages clearly. If two specimens are to be had, clean the cartilages of one, and use the other for the soft parts, making a longitudinal section of it when necessary (at $\$ 379$ ). 
Examine first the cartilages and then the soft parts, making out :

374. The thyroid (Fig. 64, th), a large plate of cartilage, consisting of right and left alæ united to one another ventrally at an obtuse angle : dorsally each alæ is produced into anterior and posterior cornua, small processes, the posterior of which articulates with a facet on the cricoid.

375. The cricoid $(c r)$, an annular cartilage, narrow ventrally, wide dorsally, situated immediately bebind the thyroid, the posterior cornua of which articulate with facets on the dorso-lateral regions of its outer surface : ventrally there is a considerable interval between the thyroid and the cricoid, bridged over by the crico-thyroid membrane (cr.th.m).

The anterinr ring of the trachea is often more or less transitional between the ordinary tracheal rings and the cricoid.

376. The arytenoids $(a r y)$, paired triangular cartilages articulated to facets in the postero-dorsal region of the anterior edge of the cricoid.

377. The cartilage of Santorini, small paired nodules embedded in the cornicula laryngis $(c . l a)$.

378. The epiglottis $(\rho p g)$, an obcordate plate of cartilage attached by its narrow ventral end to a facet on the inner surface of the ventral region of the thyroid.

379. The reddish mucous membrane lining the larynx, continuous behind with that of the trachea and in front wilh that of the pharynx.

3 So. The ventricles of the larynx ( $(\cdot . l a)$, paired shallow depressions on its inner surface at about the level of the arytenoids.

${ }_{3} S \mathrm{I}$. The vocal cords $(v \cdot c)$, paired folds of mucous membrane forming the posterior boundaries of the two ventricles and supported by ligaments : they extend from the arytenoids downwards to the inner face of the thyroid, where they are attached close together just posterior to the facet for the epiglottis.

${ }_{3}$ S2. The false vocal cords $(f . v . c)$, similar folds forming the anterior boundaries of the ventricles.

${ }_{3} S_{3}$. The crico-thyroid muscles, arising one on each side from the outer surface of the cricoid, and passing forwards and upwards to be inserted into the thyroid, which they serve to depress.

$3 S_{4}$. The posterior crico-arytenoid muscles, large paired muscles covering the dorsal surface of the cricoid, from which they arise, and passing outwards and forwards to be inserted into the arytenoids.

385. The arytenoid muscles, situated immediately anterior to the 
crico-arytenoids, and consisting of fibres passing transversely between the arytenoid cartilages, which are approximated by their contraction.

386. The anterior crico-arytenoid muscles, arising from the lateral regions of the cricoid and inserted into the arytenoids : to see them one of the alæ of the thyroid should be disarticulated and reflected.

387. The thyro-arytenoid muscles, also seen by reflection of the thyroid: they run parallel and external to the vocal cords, arising from the arytenoids and being inserted into the thyroid, which they elevate.

XLIX. Dissect away enough of the muscles in the regions of the shoulder and hip to make out :

388. The brachial plexus, formed by the union of the fifth to eighth cervical and of the first thoracic nerves, ${ }^{1}$ and giving off nerves to the arm and shoulder.

Besides several smaller nerves there are four chief trunks given off from the brachial plexus : ${ }^{2}(a)$ the ulnar nerve runs alongside the brachial artery, passes im nediately internal to the olecranon into the fore-arm, and then along the outer or ulnar side of the latter : $(b)$ the median nerve pasces internal to the humerus, entering the fore-arm proximal to the condyles, and takes a course along the inner or radial side of the fore-arm; both it and the ulnar nerve supply mainly the flexor muscles: (c) the musculo-spiral nerve, the largest of the four, goes to the dorsal side of the humerus and along the radial side of the fore-arm; it supplies mainly the extensor muscles: (d) the circumflex or subscapular nerve passes dorsalwards round the head of the humerus and supplies some of the muscles of the shoulder.

389. The lumbo-sacral plexus, formed by the union of the fifth to seventh lumbar and of the first to

1 There is a certain ambiguity in the usual mode of counting the spinal nerves: in the cervical region each nerve is named from the vertebra in front of which it emerges, the first or sub-occipital nerve coming out between the skull and the atlas, the eighth between the seventh cervical and first thoracic vertebrx: the remaining nerves are named from the vertebræ behind which they emerge : the first thoracic, for instance, is the nerve which makes its exit between the first and second thoracic vertebræ.

" If the muscles of the arm are to be dissected in the same specimen, the tracing of these nerves must be deferred. 
third sacral nerves, and giving off branches to the leg and hip.

Three chief nerves arise from the lumbo-sacral plexus: $(a)$ the crural nerve, passing out ventral to the pelvis and supplying the extensor (antericr) muscles of the thigh : (b) the obturator nerve, passing along the inner edge of the pubis and through the obturator foramen: and $(c)$ the sciatic nerve, the largest of the three, passing out between the ischium and the sacrum and going mainly to the flexor muscles. ${ }^{1}$

L. Remove the skin from the back and limbs, and make out the following muscles: ${ }^{2}$

390. The dorso-lumbar fascia, a strong sheet of connective tissue which covers all but the most superficial muscles of the back: it is continuous in front with the cervical fascia.

391. The trapezius (Fig. $65, \mathrm{~A}, t z$ ), a thin superficial sheet of muscle extending over a great part of the dorsal aspect of the cervical and thoracic regions: it arises in the middle dorsal line from the cervical and thoracic fascix : the fibres of its anterior part pass backwards and outwards, and are inserted into the metacromion $(\S 8 \mathrm{I})$, those of its posterior portion pass forwards and somewhat outwards, and are inserted into the dorsal half of the spine of the scapula.

392. The latissimus dorsi $(l . d)$, an extensive sheet of muscle arising partly from the dorso-lumbar fascia, partly from the three posterior ribs by as many triangular slips or digitations, which fit between or interdigitate with similar slips of the external oblique (§ $\mathrm{r} 39)$ : in its posterior part it is united with and scarcely distinguishable from the

1 If the muscles of the leg are to be dissected in the same specimen, the tracing of these nerves must be deferred.

2 It is advisable to have another specimen, well hardened in alcohol, for the muscles, as many of those described will have been destroyed by the previous dissection. 


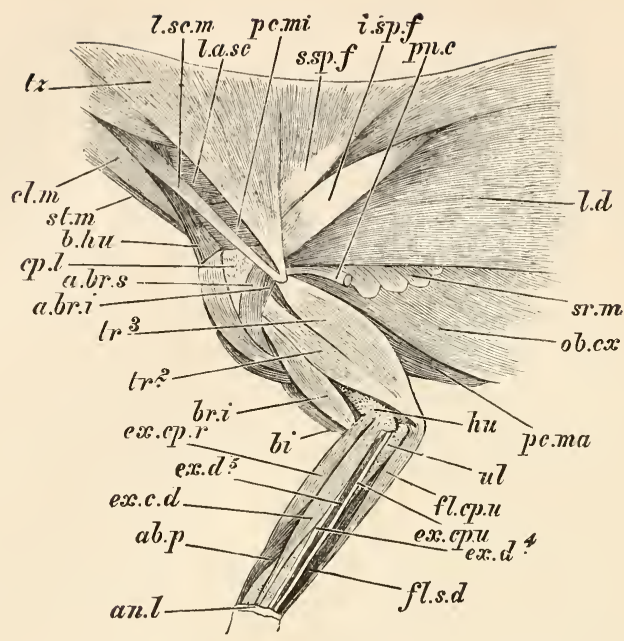

FIG. 65, A.-Lepus cuniculus. Muscles of the fore-limb, from the outer side $\left(\times \frac{1}{2}\right)$.

$a b . p$, abductor pollicis : a.br.i, abductor brachii inferior: a.br.s, abductor brachii superior : $a n . l$, annular ligament $: b . h u$, basio-humeralis : li, biceps : br.i, brachialis internus : $c l . m$, cleido-mastoid : cp.l, capsular ligament of shoulder : $c r . b r$, coraco-brachialis : $d$, deltoid : ex.c.d, extensor communis digitorum : ex.cp.r, extensor carpi radialis : ex.cp.u, extensor carpi ulnaris : ex.d.4, extensor quarti digiti : ex.d.5, extensor quinti digiti : ex.p.a, extensor parvus antibrachii : fl.ch.r, flexor carpi radialis : $f . c p . u$, flexor carpi ulnaris : $f l . p . d^{1}$, superficial head of flexor profundus digitorum, its middle portion removed to show the underlying muscles (C) : $f \cdot p \cdot d^{2}$, ulnar head of the same muscle : $f \cdot p \cdot d^{3}$, its radial head : fl.p.d $d^{4}$, its middle head: fl.s.d, flexor sublimis digitorum : $h u$, humerus : i.sp.f, infra-spinous fascia: l.a, linea alba: l.a.sc, levator anguli scapulæ: l.d, latissimus dorsi : l.sc.m, levator scapulæ major : ob.ex, obliquus externus abdominis : $p c . m a$, pectoralis major:pc.mi, pectoralis minor : $p c . t$, pectoralis tenuis : $p l$, palmaris: $p n . c$, panniculus carnosus: pr.t, pronator teres : $r . a b$, rectus abdominis: $r h . c$, rhomboideus cervicalis : rh.d, rhomboideus dorsalis : $s r . m$, serratus magnus: s.sc, subscapularis : s.sp, supra-spinatus : s.sp.f, supra-spinous faccia : st.hy, sterno-hyoid: st.m, sterno-mastoid: te.ma, teres major: $t r$, trachea : $t r^{1}$, middle, $t r^{2}$, external, $t r^{3}$, internal, and $t r^{4}$, accessory, head of triceps: $t z$, trapezius : $u l$, ulna. 
trapezius : its fibres are gathered into a comparatively narrow bundle and inserted into the humerus.

393. The levator scapulæ major (l.sc.m), a narrow band, arising from the base of the skull, and passing backwards parallel and close to the antero-external border of the trapezius, to be inserted with the latter into the metacromion.

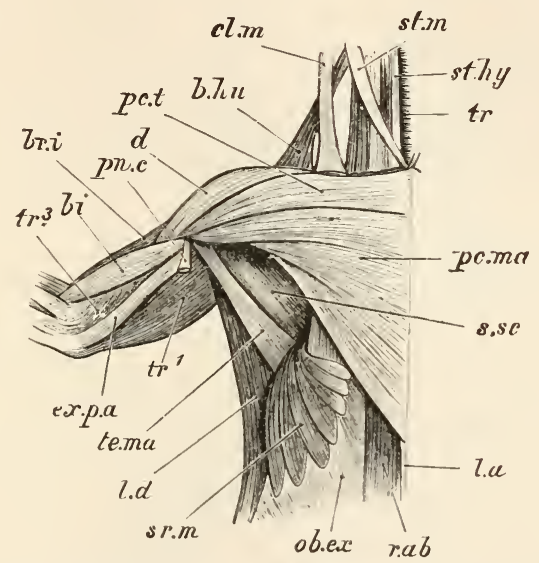

FIG. 65, B.-Lepus cuniculus. Muscles of the fore-limb, from the ventral aspect $\left(\times \frac{1}{2}\right)$. For references see Fig. 65, A.

394. The cleido-mastoid (Fig. 65, A and B, cl.m), arising with the sterno-mastoid (st.m, $\S x_{30}$ ), from the periotic, and passing backward to be inserted into the clavicle.

395. 'The basio-humeralis (b.hu), lying dorsal to the sterno- and cleido-mastoid : it arises from the basioccipital, and passes backwards and outwards to be inserted into the outer third of the clavicle.

396. The deltoid (d), a somewhat triangular muscle covering the point of the shoulder : it arises from the outer 
half of tine clavicle, and is inserted into the deltoid ridge of the humerus, its insertion covering that of the pectoralis (§ I 3 I).

LI. Cut through the trapezius, latissimus, and levator scapulæ major near their insertions, and reflect them so as to expose the underlying muscles : note :

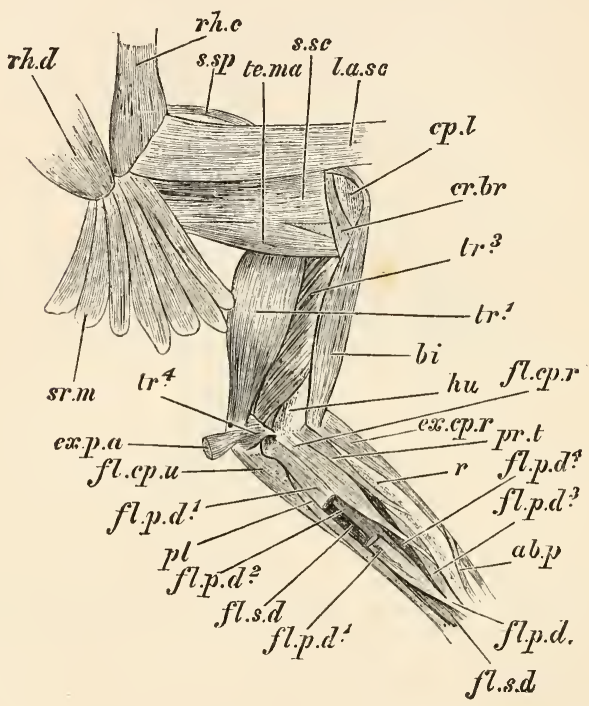

FIG. 65 , C.-Lepus cuniculus. Muscles of the fore-limb, from the inner side $\left(\times \frac{1}{2}\right)$. For references see Fig. 65, A.

397. The rhomboideus dorsalis (Fig. 65, C, rh.d), a squarish muscle, arising from the spines of the anterior thoracic vertebræ, and passing outwards to be inserted into the supra-scapular border of the scapula ( $(8 \mathrm{I})$.

398. The rhomboideus cervicalis (rh.c), a long muscle arising from the hinder surface of the skull and 
from the spines of the cervical vertebræ, and inserted with the preceding muscle into the supra-scapular border.

399. The serratus magnus (A, B, and C, sr.m), a somewhat quadrate muscle arising from the third to ninth vertebral ribs near their junctions with the sternal ribs by seven slips which interdigitate with the external oblique : its fibres pass upwards and forwards, and are inserted into the supra-scapular border.

400. The levator anguli scapulæ (l.a.sc), a large muscle arising from the transverse processes of the last five cervical vertebræ, and passing backwards to be inserted into the inner surface of the scapula close to its supra-scapular border.

40I. The pectoral muscles have already been seen (p. $290 \$ \S \mathrm{I} 3 \mathrm{I}-\mathrm{I} 33$ ); the insertion of the pectoralis minor can now be conveniently made out: the muscle passes dorsalwards, beneath (posterior to) the clavicle, into which some of its fibres are inserted, and then spreads out into a broad sheet which covers the anterior region of the scapula, and is inserted into the pre-scapular fascia, which invests the remaining muscles in the pre-scapular fossa.

402. The erector spinæ, a complicated, longitudinal muscle, forming with its fellow the ridge of the back, and serving as the chief straightener of the vertebral column.

403. The abductor brachii superior (a.br.s), a small triangular muscle arising from the acromion, and inserted into the deltoid ridge : detach from its origin and reflect.

LII. Cut through the insertions of the pectoralis minor, rhomboidei, serratus magnus, and levator anguli scapulæ: cut through the ligament joining the clavicle to the scapula, and so detach the fore-limb with the scapula from the trunk. Dissect out: 
On the inner surface of the scapula.

404. The teres major (te.ma), a stout muscle arising from the greater part of the glenoid border of the scapula, and inserted into the shaft of the humerus near the insertion of the latissimus: cut it through the middle and reflect both ends.

405. The subscapularis (s.sc) a large flat muscle arising from the whole of the subscapular fossa, and inserted into the lesser tuberosity of the humerus : detach from its origin and reflect.

406. 'The coraco-brachialis (cr.br), a small muscle covering the insertion of the subscapularis: it arises from the coracoid, and is inserted into the proximal end of the shaft of the humerus : cut through and reflect.

On the outer surface of the scapula.

407. The supra-spinatus (s.sp), arising from the whole of the pre-scapular fossa and pre-axial surface of the spine of the scapula, and inserted into the upper margin of the greater tuberosity of the humerus: detach from its origin and reflect.

f08. The abductor brachii inferior (a.br.i) arising from the infra-spinous fascia (i.sp.f) or connective tissue covering the infra-spinatus ( $(409)$, as well as from the acromion and metacromion: it passes beneath the latter and is inserted into the outer face of the humerus just distal to its head: detach from its origin and reflect, removing with it the metacromion.

409. The infra-spinatus $(i . s p)$, lies beneath the preceding: it arises from the whole post-scapular fossa and from the post-axial surface of the spina scapulæ, passes beneath the acromion, and is inserted into the greater tuberosity of the humerus: detach from its origin and reflect. 
410. The teres minor, a small muscle exposed by the removal of the preceding: it arises from the ventral third of the glenoid border of the scapula, and is inserted into the greater tuberosity just below the insertion of the infraspinatus.

In the upper arm.

4II. The extensor parvus antibrachii (ex.p.a), a small flat muscle lying on the inner face of the upper arm about midway between its anterior and posterior borders: it arises by a flat tendon from the fascia of the upper arm, and is inserted into the olecranon: cut it through and reflect.

412. The triceps, or chief extensor of the fore-arm: a very large muscle consisting of four parts or heads-

(a). The middle or long head (anconeus longus, $t r^{1}$ ), a large fleshy mass forming the posterior edge of the upper arm : it arises from the ventral third of the glenoid border of the scapula, and is inserted into the olecranon : divide and reflect.

(b). The short or outer head (anconeus brevis, $t r^{2}$ ) is situated on the outer face of the upper arm just in front of the preceding: it arises from the outer surface of the shaft of the humerus, and is inserted with the long head into the olecranon: detach from its origin and reflect.

(c). The inner head (anconeus internus, $t r^{3}$ ) occupies a similar position on the inner face of the upper arm, lying between the anconeus longus and the biceps $(\S 4 \mathrm{I} 3)$ : it arises from the posterior surface of the shaft of the humerus, and is inserted into the olecranon: detach from its origin and reflect.

$(d)$. The accessory head (anconeus quartus, $t r^{4}$ ) is a very small, fan-shaped muscle, exposed by reflection of the anconeus longus and internus, on the inner face of tha 
elbow-joint : it arises from the inner condyle of the humerus, and is inserted into the olecranon.

413. The biceps brachii (bi), or chief flexor of the fore-arm: a spindle-shaped muscle forming the anterior edge of the upper arm: it arises by a single cord-like tendon, working in the bicipital groove of the humerus, from the anterior edge of the glenoid cavity, and is inserted into the proximal end of the radius: cut it through and reflect.

414. The brachialis internus (br.i), a flattish muscle lying on the outer face of the upper arm between the anconeus brevis and the biceps: it arises from the outer surface of the proximal portion of the shaft of the humerus and is inserted into the radius just proximal to the insertion of the biceps

In the fore-arm and hand.

4I5. The extensor carpi radialis (Figs. 65, A and C, and 66, ex.cp.r), or chief extensor of the entire hand, a long muscle forming the anterior edge of the fore-arm : it arises from the outer condyle of the humerus : its distal end divides into the slender tendons which pass beneath the tendon of the abductor pollicis ( $\$ 4 \mathrm{I} 8$ ), and then under the annular ligament (an.l), a transverse fibrous band at the junction of the fore-arm and hand, and are inserted into the proximal ends of the second and third metacarpals : cut through and reflect.

4I6. The extensor communis digitorum (ex.c.d), or principal extensor of the fingers, a long muscle situated on the external and dorsal surface of the fore-arm in the groove between the radius and ulna: it arises from the outer condyle of the humerus, and at the distal end of the fore-arm divides into four tendons which pass beneath the annular ligament to the four post-axial digits. Fach tendon passes 
along the dorsal aspect of the corresponding digit, broadens out into a sort of sheath over the metacarpo-phalangeal

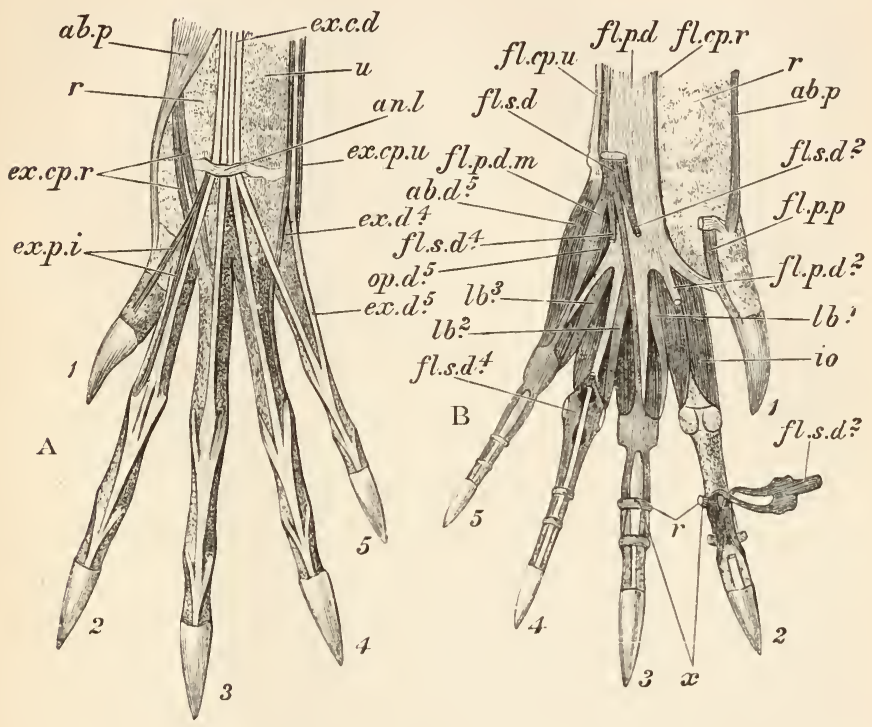

FIG. 66.-Lepus cuniculus. Muscles of the manus. A, dorsal ; $B$, ventral (nat. size).

$\mathbf{I}-5$, the digits : $a b . d .5$, abductor minimi digiti : $a b . p$, abductor pollicis : an.l, annular ligament : ex.c.d, extensor communis digitorum : ex.cp.r, extensor carpi radialis : ex.cp.u, extensor carpi ulnaris : ex.d.4, extensor quarti digiti : ex.d.5, extensor quinti digiti ; ex.p.i, extensor pollici; et indicis: $t_{.} p . d . m$, flexor brevis minimi digiti : $f_{.} p . p$, flexor brevis pollicis : $f . c p . r$, flexor carpi radialis : $f . c p . u$, flex or carpi ulnaris : $f . p . d$, flexor profundus digitorum : fl.t. $d^{2}$, its tendon to the index digit, cut short : fl.s.d, flexor sublimis digitorum: fl.s. $d^{2}$, its tendon to the second digit partly removed, and its distal end reflected : f.s. $d^{4}$, its tendon to the fourth digit partly removed, and having the sheath it forms over the metacarpo-phalangeal articulation cut through and reflected right and left: io, interossei : $l b .1, l b .2, l b .3$, lumbricales : $r$ (above), radius : $r$ (below), retinacula, those of the second digit cut through and reflected to show the insertion of the flexor sublimis : $u$, ulna. 
and inter-phalangeal articulations, and is inserted into the middle and distal phalanges : divide and reflect.

4I7. The extensor pollicis et indicis (ex.p.i), a small, slender muscle lying in the groove between the radius and ulna, beneath the common extensor: it arises from the radius: its tendon enters the manus along with that of the extensor communis, and divides into two, one of which is inserted into the ungual phalanx of the pollex, the other into the distal end of the metacarpal of the index.

4I 8 . The abductor pollicis ( $a b . p$ ), arising from the outer surface of the shaft of the radius: its tendon crosses that of the extensor carpi radialis, and is inserted into the metacarpal of the pollex.

419. The extensor quarti digiti (ex.d.4), the extensor quinti digiti (ex.d.5), and the extensor carpi ulnaris (ex.cp.u), three small, slender muscles arising close together from the external condyle of the humerus, and passing backwards behind the ulna to the manus. The extensor quarti digiti is inserted into the ungual phalanx of the fourth digit, the extensor quinti digiti into the base of the metacarpal and the proximal phalanx of the fifth digit, and the extensor carpi ulnaris into the proximal end of the fifth metacarpal: cut through and reflect all three muscles.

420. The flexor carpi ulnaris (fl.cp.u), forming the posterior edge of the fore-arm: it arises by an expanded head from the inner face of the olecranon, and is inserted into the pisiform: divide and reflect.

42I. The pronator teres (Fig. 65, C, pr.t), a small muscle arising from the inner condyle of the humerus, and inserted by a long tendon into the middle of the inner side of the shaft of the radius.

422. The flexor carpi radialis (fl.cp.r), a long, slender muscle arising from the inner condyle of the humerus just 
posterior to the origin of the pronator teres, and passing into a long tendon which is inserted into the proximal end of the second metacarpal: the insertion is best seen at a later stage: cut through and reflect.

423. The flexor profundus digitorum, or flexor perforans $(f l p . d)$, a large muscle, the chief flexor of the digits, and made up by the union of four heads-

(a). The superficial head arises from the inner condyle of the humerus just posterior to the origin of the flexor carpi radialis, and in common with the palmaris (\$ 424$)$ : cut it through near its origin and reflect.

(b). The ulnar head lies deeper than and anterior to the foregoing, by the reflection of which it is exposed: it arises from the inner condyle of the humerus in common with the flexor sublimis $(\$ 425)$.

(c). The radial head arises from the proximal part of the posterior surface of the radius.

(d). The middle head arises from the posterior surface of the ulna. The four heads unite at the distal end of the fore-arm to form a common tendon, the insertion of which is best seen at a later stage of the dissection ( $\$ 427)$.

424. The palmaris $(p l)$, a small, slender muscle arising with the superficial head of the deep flexor from the inner condyle of the humerus and passing into a long tendon which becomes lost in the palmar fascia, or sheet of connective tissue covering the ventral surface of the manus, sending off also a small slip which is inserted into the ungual phalanx of the pollex : cut through and reflect.

425. The flexor sublimis digitorum (fl.s.d), or flexor perforatus, lying immediately beneath the superficial head of the flexor profundus: it arises in common with the ulnar head of the latter from the inner condyle of the humerus, and divides distally into four tendons, which pass, superficial 
and post-axial to the tendon of the flexor profundus, into the manus and along the ventral faces of the second to the fifth digits : each tendon divides at the base of the proximal phalanx into two slips which pass one on either side of the proximal phalanx to be inserted into the proximal end of the middle phalanx : cut through and reflect.

The tendon for the fifth digit has in connection with it a very small muscle, the flexor brevis minimi digiti $(f . p . d . m$.), which arises partly from it, partly from the pisiform bone, and joins the tendon again at its distal end. A similar muscle, the flexor brevis pollicis $(f . p p$.$) , arises from the annular ligament and is inserted into the tendon$ of the flexor profundus going to the pollex.

426. The retinacula $(r)$, transverse tendinous bands which keep the flexor tendons in place: there is one at the metacarpo-phalangeal articulation, formed by an expansion of the tendon of the flexor sublimis, one proximal to the joint between the proximal and middle phalanges, and another distal to the same joint.

427. The insertion of the flexor profundus: its tendon spreads out into a broad stout sheath lying immediately dorsal to the tendons of the superficial flexor: from this sheet five tendons are given off, one to each digit, each passing along the ventral face of the digit and being inserted into its ungual phalanx: the tendons of the second to the fifth digits pass between the two slips into which the corresponding tendon of the flexor sublimis is divided.

428. The lumbricales ( $l b .1,2,3)$, three small spindle-shaped muscles arising from the ventral surface of the tendon of the flexor profundus at the place where it divides into slips for the second to the fifth digits : they are inserted into the proximal ends of the proximal phalanges on the pre-axial side of the third, fourth, and fifth digits respectively.

429. The abductor minimi digiti $\left(a b . d^{5}\right)$, a small muscle lying on the ventral face of the fifth metacarpal: it arises from the pisiform bone, and its tendon joins with that of the superficial flexor going to the fifth digit. 
430. The interossei (io), seen, by reflecting the flexor profundus with the lumbricales and the flexor minimi digiti, as three pairs of small muscles lying on the ventral faces of the second to the fourth metacarpals and one on the pre-axial side of the fifth metacarpal: they arise from the metacarpals. The two muscles of each pair are in close contact with one another for the greater part of their extent, but separate distally to be inserted into the sesamoid bones at the meta. carpo-phalangeal articulation; a small additional slip occurs on the post-axial side of the second digit, and one on each side of the fourth: these are much smaller than, and arise ventrally to, the paired muscles.

LIII. Dissect away the origins and insertions of any muscles still left attached to the shoulder-joint, and make out :

43I. The capsular ligament surrounding the shoulderjoint : it consists of thin fibrous tissue, and is attached on the one hand round the margin of the glenoid cavity and on the other round the neck of the humerus: if it has not already been cut, make an incision through it, and notice that it helps to bound a closed synovial cavity, between the two bones, lined by a delicate synovial membrane which secretes a small quantity of synovial fluid.

432. A similar synovial cavity at the elbow-joint: the joint is further strengthened by strong lateral ligaments, which pass, one on the pre-axial side from the humerus to the radius, the other on the post-axial side from the humerus to the ulna.

LIV. Make out the muscles of the hind limb, beginning with those which connect it with the trunk :

On the ventral surface of the vertebral column.

433. The psoas magnus (Fig. 67, B, ps.m), a large muscle in the lumbar region passing longitudinally backwards from the diaphragm to the thigh: it arises from the last three ribs, the last three thoracic and all the lumbar 


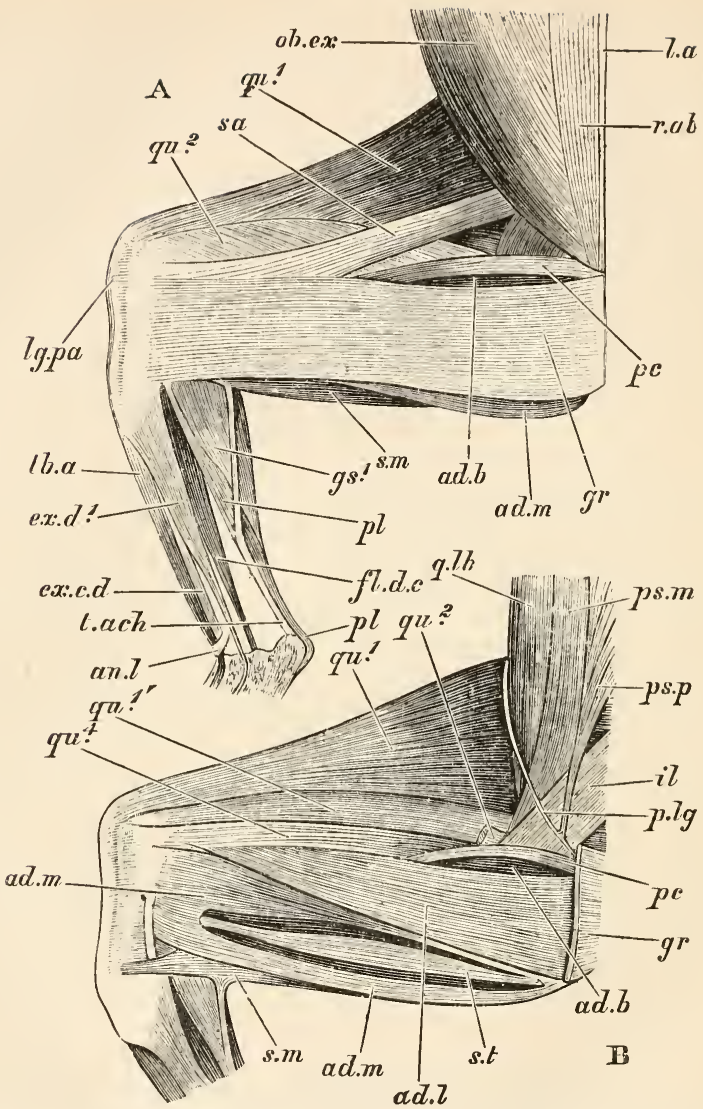

FIG. 67.-Lepus cuniculus. Muscles of the inner side of the hind limb : A, superficial, B, deep layer $\left(\times \frac{1}{2}\right)$.

In $\mathbf{A}$ the adjoining portions of the abdominal muscles are shown: in $\mathrm{B}$ these are removed so as to show the muscles on the ventral face of the vertebral column.

$a d . b$, adductor brevis: $a d . l$, adductor longus : ad.m, adductor magnus, its fibres separated in $\mathrm{B}$ to show the semi-tendinosus : ex.c.d, extensor communis digitorum : ex.d. I, extensor primi digiti : fl.d.c, flexor com- 
munis digitorum : $g r$, gracilis, renoved in B with the exception of its origin : $g s^{1}$, inner head of gastrocnemius : l.a, linea alba, lg.pa, ligamentuin patellæ: ob.ex, obliquus externus abdominis: $p c$, pectineus : $p l$, plantaris: $q . l b$, quadratus lumb rrum: $q u .1$, superficial head of rectus anticus: $q u .1^{1}$, its deep head : qu.2, vastus internus, mostly removed in $\mathrm{B}: q u .4$, crureus : $r . a b$, recrus abdominis : $s a$, sartorius, removed in $\mathrm{B}: s, m$, semi-membranosus : s.t, sımi-tendinosus : $t . a c h$, tendo Achillis : tb.a, tibialis anticus.

vertebræ: in its anterior half it forms the inner slope of the great longitudinal muscular ridge, which lies close to the middle line and has embedded in it the long transverse processes of the lumbar vertebræ: at about the middle of its course it turns outwards and is inserted into the lesser trochanter of the femur: its insertion is best seen after some of the muscles of the thigh have been removed ( $\$ 448)$.

434. The quadratus lumborum (q.lb), a large muscle lying just external to and having the same general direction as the preceding: it forms the external slope of the muscular ridge mentioned above, as well as the whole of its deeper portion : it consists of two parts separated by the transverse processes of the lumbar vertebræ, the inner arising from the last five thoracic and all the lumbar vertebræ, the outer from the five last ribs and corresponding transverse processes and from all the lumbar vertebræ: it is inserted partly into the lumbar vertebræ, partly into the pubic border of the ilium.

435. The psoas parvus ( $p$ s. $p$ ), lying internal to about the posterior half of the psoas magnus: it arises from the bodies of the four posterior lumbar vertebræ, and is inserted into the pubis by a tendon which acquires a connection with Poupart's ligament $(p . l g)$.

436. The iliacus (il), a broad muscle, arising from the ventral faces of the last lumbar and first sacral vertebræ, and passing obliquely outwards and backwards to be inserted, along with the psoas magnus, into the lesser trochanter (\$ 448 ). 
In the thigh and hip.

437. The fascia lata, or strong fibrous covering of the muscles of the thigh : it is especially thick on the outer side : dissect it away so as to expose the underlying muscles, taking care not to injure any of the latter, some of which are closely attached to the fascia.

438. The sartorius (Fig. $67, \mathrm{~A}, s a$ ), a small flat muscle on the inner face of the thigh, about midway between its anterior and posterior borders : it arises from the middle of Poupart's ligament, and passing obliquely outwards blends with the anterior edge of the gracilis ( $\$ 439)$.

439. The gracilis $(g r)$, a large thin sheet of muscle covering the greater part of the posterior half of the inner surface of the thigh: it arises from the whole of the symphysis pubis, and passes distally into a broad fascia which is inserted into the upper part of the inner (pre-axial) border of the tibia : separate it from the underlying muscles, cut it through, and reflect along with the sartorius.

440. The quadriceps (Figs. 67 and 68, qu), or great extensor of the crus, a very large muscle forming the anterior part of the thigh: it consists of several parts-

(a). The long or superficial head of the rectus anticus $(q u . \mathrm{r})$, forming the anterior edge of the thigh : it arises from the pubic border of the ilium, and is inserted by a strong thick tendon, the ligamentum patellæ (lg.pa), in which the patella is embedded, into the crest of the tibia: carefully separate it from the underlying muscles, cut through and reflect.

On the dorsal surface of the thigh the edge of the rectus is produced, near its proximal end, into a somewhat semicircular expansion which is inserted into the fascia lata: this represents what is usually a separatc muscle, the tensor vaginæ femoris or tensor fasciæ latæ.

(b). The vastus internus $(q u, 2)$, occupying the space, on 
the inner surface of the thigh, between the rectus anteriorly and the sartorius and gracilis posteriorly: it arises from the

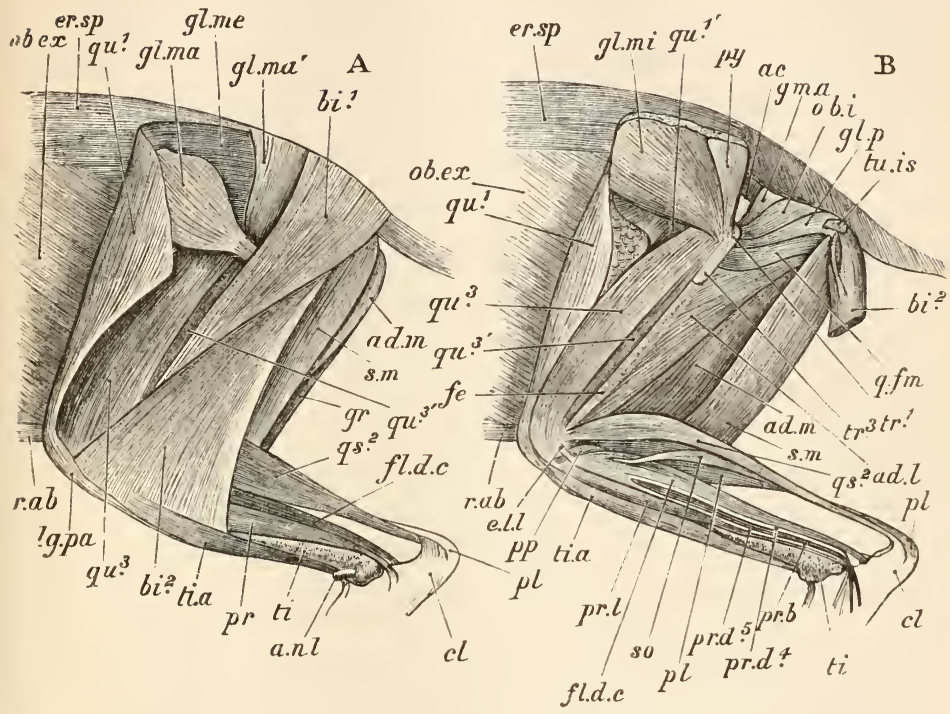

FIG. 68.-Lepus cuniculus. Muscles of the outer side of the hind limb : A, superficial, B, deep layer $\left(\times \frac{1}{2}\right)$.

$a c$, acetabulum: ad.l, adductor longus: $a d . m$, adductor magnus : an.l, annular ligament: $\mathrm{bi}^{\mathrm{l}}$, superficial head of biceps femoris : $b i^{2}$, its deep head, reflected in $B$ to show its double origin: $\mathrm{cl}$, calcaneum : e.l.l, external lateral ligament of knee : er.sp, erector spinæ: f.d.c, flexor communis digitorum: $g l . m a, g l . m a$, gluteus maximus : gl.me, gluteus medius: $s l . m i$, gluteus minimus: gm.a, gemellus anterior: $g l \cdot p$, gemellus posterior: $g r$, gracilis : $q s^{2}$, outer head of gastrocnemius, turned aside in $B$ to show the soleus: $l_{5} \cdot p a$, ligamentum patellæ: ob.ex, obliquus externus: $o b . i$, obturator internus : $p l$, plantaris $: p p$, popliteus : pr, peronei (in A) : pr.b, peroneus brevis : pr.d.4, peroneus quarti digiti : pr.d.5, peroneus quinti digiti : pr.l, peronens iongus : $p y$, pyriformis : $q u . \mathrm{I}$, superficial head of rectus anticus $: q u . \mathrm{I}^{1}$, its deep head: $q u \cdot 3$, superficial head of vastus externus : $q u \cdot 3^{1}$, its deep head: q.fm, quadratus femoris: $r a b$, rectus abdominis : s.m, semi-membranosus: so, soleus: ti, tibia: ti.a, tibialis anticus: tr.I, great trochanter: $t r \cdot 3$, third trochanter: tu.is, tuber ischii. 
neck of the femur, and at its distal end joins the rectus in the ligamentum patellæ: cut through and reflect.

(c). The principal or superficial head of the vastus externus (qu.3), situated just posterior to the rectus anticus on the outer surface of the thigh: it is the largest head of the quadriceps : it arises from the neck of the femur and from the great trochanter, and passes distally into the conimon tendon of the quadriceps or ligamentum patellæ.

(d). The short or deep head of the rectus anticus $\left(q u . \mathrm{I}^{1}\right)$, a cylindrical muscle lying along the inner or pre-axial side of the vastus externus, by which it is partly enwrapped: it arises from the posterior part of the pubic border of the ilium, and passes distally into the common tendon of the quadriceps.

(e). The accessory or deep head of the vastus externus $\left(q u \cdot 3^{1}\right)$, a flattish muscle, lying between the concave inner face of the superficial head and the crureus (see below): it arises from the femur close to the origin of the superficial head, and distally joins the ligamentum patellæ: cut through and reflect both heads of the vastus externus and the short head of the rectus.

$(f)$. The crureus (qu.4), arising from the shaft of the femur, the anterior (dorsal) surface of which it covers, and passing distally into the commom tendon of insertion of the quadriceps : detach it from its origin and reflect.

44I. The biceps femoris $(b i)$, covering the posterior half of the outer side of the thigh and forming the chief flexor of the crus : it has two heads, of which the first or anterior head $\left(b i^{1}\right)$ arises by a wide flat tendon from the three posterior sacral and the three anterior caudal vertebræ, and passes outwards, gradually diminishing in breadth and becoming sub-cylindrical : the second or posterior head $\left(b i^{2}\right)$ arises from the posterior part of the tuber ischii in 
common with the adductor magnus ( $\$ 443$ ), and passes outwards, widening as it goes : the two heads unite, and are inserted by a strong and extensive fascia into the distal end of the femur and proximal end of the tibia. Cut through both heads and reflect: it will be seen that the posterior head has a second origin in the form of a flat tendon arising in common with the semi-membranosus $(\S 442)$ from the anterior end of the tuber ischii.

442. The semi-membranosus (s.m), forming the posterior edge of the thigh : it arises from the tuber ischii in common with the accessory tendon of the posterior head of the biceps, and is inserted by the same fascia as the gracilis into the proximal part of the pre-axial border of the tibia: from the distal end of its posterior edge proceeds a long tendon which passes along the inner side of the shank and joins the tendo Achillis ( $\$ 460)$ : cut through and reflect.

443. The adductor magnus $(a d . m)$, a large fleshy muscle in the posterior region of the thigh, fully exposed by the removal of the gracilis, which covers its inner face, and of the semi-membranosus and biceps, which cover its outer face: it arises from the posterior edge of the ischium and tuber ischii, and is inserted into the inner side of the distal end of the femur and inner condyle of the tibia.

444. The semi-tendinosus (s.t), seen by separating the fibres of the adductor magnus in which it is embedded: it is a long spindle-shaped muscle, arising from the tuber ischii and inserted by a long tendon, which emerges from the adductor magnus near the distal end of its outer surface, into the inner condyle of the tibia : cut through and reflect both semi-tendinosus and adductor magnus.

445. The adductor longus ( $a d . l)$, a large, somewhat triangular muscle, forming the main part of the mass of flesh still left posterior to the femur: it arises from the whole of 
the symphysis pubis, and is inserted into about the middle third of the shaft of the femur.

446. The adductor brevis ( $a d . b)$, a small, flat muscle situated just anterior to the inner two-thirds of the preceding: it arises from the anterior end of the symphysis pubis, and is inserted by a broad tendon into the shaft of the femur.

447. The pectineus $(p c)$, a small muscle situated immediately in front of the preceding, which it resembles in shape and size: it arises from the pubis just in front of the symphysis, and is inserted into the shaft of the femur: cut through and reflect it as well as the adductores brevis and longus.

448. The insertions of the psoas magnus $(\S 433)$ and iliacus ( $\$ 436)$ are now exposed : divide both muscles near their insertion and reflect.

449. The gluteus maximus ( $g l . m a)$, covering the outer or gluteal surface of the ilium: it arises by a broad fascia from the ischial border of the ilium and from the sacrum : anteriorly it becomes inseparably united with the rectus anticus, posteriorly its origin is covered by the anterior head of the biceps: it consists of two parts, one lying mainly external to the pubic border of the ilium and having its fibres directed longitudinally, the other at about the level of the acetabulum and having its fibres directed transversely: the two portions unite with one another and are inserted into the third trochanter: detach from its origin and reflect.

450. The gluteus medius ( $g l . m e)$, exposed by removal of the preceding: it arises from the supra-iliac and iliac borders of the ilium and from the sacrum: its fibres pass directly backwards, and are inserted into the great trochanter : detach from its origin and reflect. 
45r. The gluteus minimus ( $g$ l.mi), exposed by the reflection of the preceding: it arises from the whole gluteal fossa of the ilium from its supra-iliac and iliac borders and from the first sacral vertebra: it is inserted into the great trochanter

452. The pyriformis $(f y)$, a triangular muscle, lying immediately posterior to the gluteus minimus : it arises from the second and third sacral vertebræ, and passes directly outwards to be inserted into the great trochanter, beneath the insertion of the gluteus medius, and posterior and dorsal to that of the gluteus minimus : divide and reflect: also detach the gluteus minimus from its origin and reflect.

453. The quadratus femoris (q.fm), a small stout muscle lying parallel to the inner edge of the adductor longus: it arises from the tuber ischii, and is inserted into the posterior (ventral) side of the shaft of the femur at about the level of the third trochanter : divide and reflect.

454. The obturator internus (ob.i), a flat muscle arising from the dorsal or inner aspect of the obturator foramen, and passing first upwards, then outwards and forwards, to be inserted into the trochanteric fossa: to see its origin the caudal vertebræ should be pressed aside.

455. The gemelli, two very small muscles in connection with the preceding : the gemellus anterior $(g m . a)$ arises from the dorsal edge of the ischium and partly covers the anterior edge of the obturator internus; the gemellus posterior $(g l . p)$ arises from the tuber ischii and covers its posterior border : both are inserted into the tendon of the obturator internus.

456. The obturator externus, arising from the outer or ventral aspect of the obturator foramen, and inserted by a strong tendon into the trochanteric fossa: detach the obturatores from their origin and reflect. 
In the shank and foot.

457. The tibialis anticus (Figs. 67,68 , and 69, tb.a), forming the anterior border of the shank: it arises from the outer tuberosity of the tibia and from its cnemial crest, and is continued distally into a long tendon, which passes under a strong annular ligament (an.l) placed obliquely at the distal end of the anterior surface of the femur : entering the foot the tendon of the tibialis anticus is inserted into the proximal end of the second (apparent first) metatarsal : divide and reflect, first separating carefully from the next muscle.

458. The extensor communis digitorum (ex.c.d), situated between the tibia and the tibialis anticus, and closely applied to the latter : it arises by a long tendon from the anterior surface of the distal end of the femur just external to the intercondylar notch: to see this origin the tendon must be traced through the capsule of the kneejoint (§ 474): distally the muscle divides into four tendons, which pass through the annular ligament, then through a similar but smaller loop on the dorsal surface of the carpus, and are inserted into the phalanges of the four digits in the same manner as the corresponding muscle of the hand $(\S 4 \mathrm{I} 6)$ : divide and reflect.

459. The extensor digiti primi (properly secondi) (ex.d.r), arising from the inner tuberosity of the tibia, and passing along the inner aspect of the crus, at about the middle of which it becomes tendinous : this tendon runs in a groove on the pre-axial side of the distal end of the tibia, beneath (ventral to) the curved proximal end of the second metatarsal, curves over to the dorsal side of the latter, and unites with the first tendon of the common extensor on the dorsal surface of the proximal phalanx : divide and reflect.

460. The gastrocnemius (qs), a large two-header 


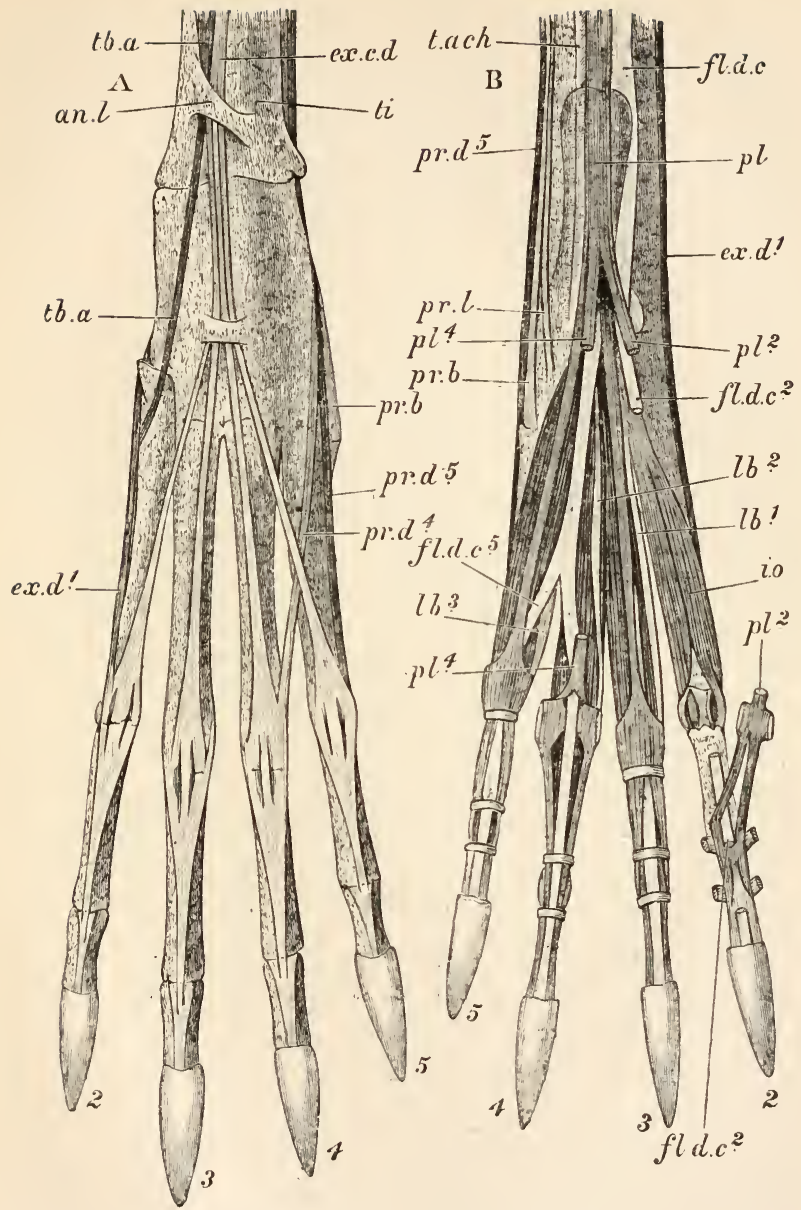

Fig. 69.-Lepus cuniculus. Muscles of the pes: A, dorsal, $B$, ventral (nat. size).

2-5, the digits: ex.c.d, extensor communis digitorum: ex.d.r, extensor primi (properly secondi)digiti : fl.d.c, flexor communis digitorum: 
fld.c. its tendon to the second digit, in great part removed to show the interossei : $f . d . c^{5}$, its tendon to the fifth digit: i.o, interossei : $l b . I$, $l 6.2, l b .3$, lumbricales: $p l$, plantaris : $p l^{2}$, its tendon to the second digit, partly removed and with its distal end reflected : $p l^{4}$, its tendon to the fourth digit, partly removed, and having the sheath it forms over the metatarso-phalangeal articulation slit up and reflected right and left: $p r . b$, peroneus brevis : $p r . d .4$, peroneus quarti digiti : pr.d.5, peroneus quinti digiti : pr.l, peroneus longus : t.ach, tendo Achillis, inserted into the calcaneum : $t b . a$, tibialis anticus : $t i$, tibia.

muscle forming the posterior surface of the shank: its inner head $\left(q s^{1}\right)$ arises from the inner condyle of the femur and pre-axial fabella ( $\$ 94)$, its outer head $\left(q s^{2}\right)$ from the outer condyle and post-axial fabella : the two heads unite with one another and with the soleus $\left(\S_{46 I}\right)$ to form a large strong tendon, the tendo Achillis, which is inserted into the extremity of the calcaneum.

46I. The soleus (so), a small muscle seen by turning aside the outer head of the gastrocnemius; it arises by a long tendon from the head of the fibula, and joins the gastrocnemius to form the tendo Achillis.

462. The plantaris $(p l)$, a large muscle, the proximal fleshy part of which is enwrapped by the gastrocnemius, from which it must be carefully separated: by dividing and reflecting the gastrocnemius and soleus, the plantaris is seen to arise from the posterior surface of the femur immediately proximal to the outer condyle and from the post-axial fabella : at about the middle of the crus it passes into a strong flat tendon, which curves round to the posterior side of the tendo Achillis and over the end of the calcaneum to reach the sole of the foot: the tendon divides into four slips, which are related to the digits in the same way as those of the flexor sublimis to the digits of the manus $(\$ 425)$ : divide and reflect.

463. The popliteus $(p p)$, or rotator of the knee, lying obliquely across the back of the knee-joint: it arises by 
a strong tendon from the outer condyle of the femur, passes backwards beneath the external lateral ligament ( $\$ 474)$, and curves round the posterior surface of the proximal end of the tibia to be inserted into the pre-axial edge of that surface : divide and reflect.

464. The flexor digitorum communis (fl.d.c), closely applied to the posterior surface of the tibia: it arises from the outer tuberosity of the tibia and from the head of the fibula: its tendon enters the foot to the inner or pre-axial side of the calcaneum, then expands and divides into tivo, each subdivision soon dividing again: the four slips thus formed have the same relation to the digits as those of the flexor profundus to the digits of the manus ( $\$ 427$ ).

465. The lumbricales $(l b)$, three very small spindle-shaped muscles arising from the tendon of the preceding, and inserted on the pre-axial sides of the proximal ends of the proximal phalanges of the third, fourth, and fifth digits respectively.

466. The peroneus longus (pr.l), a long, slender muscle arising from the outer tuberosity of the tibia: it passes into a long tendon which traverses a groove on the post-axial side of the distal end of the tibia, and is inserted into the cuboid : divide and reflect.

467. The peroneus brevis $(p r . b)$, arises from the outer tuberosity of the tibia, partly united with the peroneus quarti digiti; its tendon accompanies that of the peroneus longus, and is inserted into the proximal end of the fifth (last) metatarsal.

468. The peroneus quarti digiti (pr.d.4), arises from the shaft of the fibula, being united at its origin with the preceding: its tendon enters the foot with those of the other peronei, and is inserted into the distal end of the fourth (apparent third) metatarsal.

469. The peroneus quinti digiti $(p r \cdot d .5)$, arises from 
the head of the fibula, united with the preceding: its tendon passes with those of the other peronei into the foot, and is inserted into the distal end of the last metatarsal.

470. The interossei $(i .0)$ have the same general disposition as those of the manus ( $\$ 430$ ), there being a pair on the ventral or plantar side of each metatarsal: there are also two very slender slips, one on the post-axial side of the second (apparent first), another on the pre-axial side of the fourth metatarsal.

LV. Dissect away the origins and insertions of any muscles still left attached to the hip and kneejoints, and make out :

47 I. The capsular ligament of the hip-joint, a membrane attached on the one hand just external to the rim of the acetabulum and on the other round the neck of the femur: partly remove it so as to see the synovial cavity which it helps to enclose.

472. The ligamentum teres, exposed by the severance of the capsular ligament: it is a strong band of fibres passing from the bottom of the acetabulum to the pit on the head of the femur ( $\$ 93$ ).

473. The cotyloid ligament, a rim of fibro-cartilage attached round the edge of the acetabulum.

474. The capsular ligament of the knee-joint, bourding its synovial cavity, which will have been opened in tracing the tendon of the extensor communis digitorum : it is strengthened in front by the ligamentum patellæ already seen ( $\$ 440$ ), and at the sides by the internal and external lateral ligaments, which pass respectively from the inner and outer tuberosities of the femur to the corresponding tuberosities of the tibia.

475. The inter-articular ligaments, extending between the actual articular surfaces of the femur and tibia, 
and connecting the semi-lunar cartilages ( $\$ 97)$ with one or other bone.

The posterior ligament extends from the inner (post-axial) face of the internal condyle of the femur obliquely downwards to about the middle of the posterior edge of the articular surface of the tibia: the anterior crucial ligament extends from the inner (pre-axial) face of the external condyle of the femur to the anterior part of the articular surface of the tibia, near the middle line: the posterior crucial ligament extends from a point within the external condyle of the femur and immediately proximal to the attachment of the posterior ligament, obliquely downwards and outwards to the posterior end of the postaxial semi-lunar cartilage; it is continued from the anterior end of the same cartilage, passes inwards in front of the tibial attachinent of the anterior crucial, and is attached near the inner border of the articular surface of the tibia : the internal or pre-axial semi-lunar cartilage has a ligament at its anterior end which crosses in front of the posterior crucial to be inserted into the articular surface of the tibia near the middle of its anterior region; a similar ligament from the posterior end of the cartilage is inserted immediately in front of the tibial attachment of the posterior ligament.

LVI. In the brain, dissect off the pia mater, taking special care not to remove the nerve roots (\$\$ 488-495) or the arteries ( $\$ 476)$ with it: the latter may, however, be removed as soon as seen. Make out the following points without further dissection : ${ }^{1}$

476. The circle of Willis, an arrangement of arteries on the base of the brain, due to the anastomosis of the vertebrals and internal carotids, and forming a sort of hexagonal figure round the optic chiasma ( $\$ 486)$, infundibulum $\left(\S_{4} 8_{5}\right)$, and corpus mammillare $\left(\S_{4} 87\right)$.

The vertebral arteries, entering the foramen magnum, unite on the ventral surface of the medulla oblongata $(\$ 477)$ to form the median

1. It is best, if possible, to dissect two brains simultaneously, one fresl, the other well hardened : if only one is used, it should be only moderately hardened-say in alcohol of about 70 per cent. 
longitudinal basilar artery : this passes forwards, sending off branches to the medulla oblongata and cerebellum ( $\$ 478$ ), and just anterior to the pons Varolii ( $\$ 479$ ) bifurcates, forming the paired posterior cerebral arteries : the internal carotids reach the base of the brain at about the level of the optic chiasma, and each divides into the anterior cerebral artery directed inwards and forwards, and the middle cerebral directed outwards : the "circle" is completed by the two anterior cerebrals uniting to form a median trunk which passes forwards between the cerebral hemispheres, and by the union of each internal carotid to the corresponding posterior cerebral by a slender posterior communicating artery.

477. The medulla oblongata, directly continuous with the spinal cord through the foramen magnum, and resting, when in the skull, on the basi-occipital. It is composed externally of white matter, and is marked on its upper and lower surfaces respectively by the dorsal and ventral fissures, continuous with the similar and similarly named structures in the spinal cord: on each side of the ventral fissure is a narrow longitudinal elevation, the ventral pyramid (Fig. $70, v \cdot p$ ), immediately external to the anterior part of which is a band of transverse fibres, the corpus trapezoideum (c.tz): posterior to each corpus trapezoideum. and bounded internally by the ventral pyramid, is an area (ol.b) answering to the olivary body of the higher mammalian brain: on each side of the dorsal fissure is the narrow dorsal pyramid (Fig. $7 \mathbf{I}, d . p$ ), of which at present only a small part is seen (see $\$ 522$ ): external to this, and forming the dorso-lateral region of the medulla, is the corpus restiforme (c.rs).

478. The cerebellum $(c b)$, on the dorsal side of the medulla oblongata; a greatly convoluted body, composed externally of grey matter, and consisting of a central lobe or superior vermis $\left(c b^{1}\right)$, two lateral lobes $\left(c b^{2}\right)$, and two irregular globular bodies (flocculi, $f$ ), each of which 
projects laterally outwards from the corresponding lateral lobe, and fits into a deep pit in the cranial wall of the periotic bone $(\$ 56)$.

479. The pons Varolii (p. $\left.\tau^{\prime} a\right)$, a strong band of white fibres running transversely across the anterior part of the

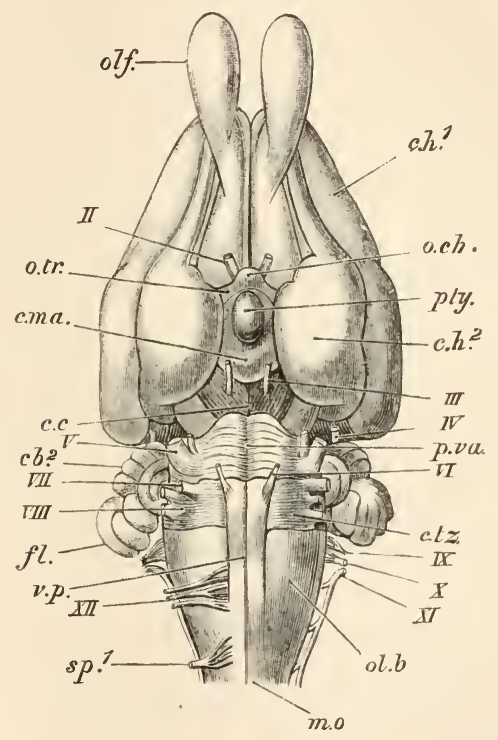

FIG. 70.-Lepus cuniculus. The brain from beneath (nat. size). $c b^{2}$, lateral lobe of cerebellum: $c . h^{1}$, frontal, and $c \cdot h^{2}$, temporal, lobe of central hemisphere : c.ma, corpus mammillare : c.tz, corpus trapezoideum : $f$, flocculus : $m . o$, medulla oblongata : o.ch, optic chiasma : ol.b, olivary body : olf, olfactory lobe : o.tr, optic tract : pty, pituitary body: $p . v a$, pons Varolii : v.p, ventral pyramid : $I I-X I I$, roots of cerebral nerves : $s p^{1}$, first spinal nerve.

ventral surface of the medulla oblongata immediately in front of the ventral pyramids and corpora trapezoidea: it ends in front in a forwardly curved margin, and is marked 
by a median groove, continuous with that of the medulla, and lodging the basilar artery. Laterally, the fibres of the pons bend upwards and enter the cerebellum, forming its middle peduncles.

480. The crura cerebri (c.c), two strong diverging bands of white fibres on the ventral surface of the brain, proceeding forwards and outwards from the anterior edge of the pons Varolii.

$48 \mathrm{r}$. The cerebral hemispheres (c./), two large masses which constitute the greater part of the brain: they are closely applied to one another along their flat inner faces; broad and somewhat truncated posteriorly (parietal lobes), where they abut against the cerebellum; produced into a blunt point anteriorly (anterior or frontal lobes, $\left.c . h^{1}\right)$; and produced downwards into the prominent temporal lobes $\left(c . h^{2}\right)$ which partly overlap the crura cerebri. Where the temporal becomes continuous, ventrally, with the frontal lobe is a slight angulation, the rudimentary Sylvian fissure. The surface of the hemispheres is formed of grey matter, and is smooth except for one or two slight depressions or sulci.

482. The corpus callosum $(c p . c l)$, seen, by slightly separating the hemispheres from one another above, as a strong white transverse band, connecting them with one another along the middle third of their length.

483. The pineal body $(p n)$ and a portion of the optic lobes $\left(o . l^{1}, o . l^{2}\right)\left(\S \S 5^{1} 7\right.$ and 519$)$ are seen on the dorsal surface of the brain, between the cerebrum and cerebellum.

484. The olfactory lobes (olf), two knob-like bodies of grey matter proceeding forward from the anterior part of the ventral surface of the hemispheres, and connected by a strong white band with the anterior edge of the temporal lobe. They fit into the deep olfactory fossæ of the skull, 
and from their lower and front surfaces are given off the filaments of the first or olfactory nerve.

485. About the centre of the base of the brain, between the temporal lobes of the cerebral hemispheres, is a slight elevation pierced in the centre by a small aperture; the elevation is the infundibulum; to it is attached in the perfect state of the brain the oval, vascular pituitary body (pty), which in removing the brain is almost always left behind in the sella turcica $(\$ 48)$.

486. Immediately in front of the infundibulum is a strong, curved, transverse band of white fibres, giving off from its convex anterior margin two strong nerves. The latter are the optic nerves $(I I)$ : the transverse band is constituted by the two optic tracts (o.tr), which, together with the nerves, form the optic chiasma (o.ch).

487. The corpus mammillare (c.ma), a rounded elevation immediately posterior to the infundibulum, in the angle between the crura cerebri.

488. The oculomotor nerves (third pair, $I I I$ ), arising one from each crus cerebri, slightly posterior to the corpus mammillare.

489. The pathetic nerves (fourth pair, $I V$ ), arising from the anterior part of the dorsal surface of the medulla oblongata (valve of Vieussens, $\$ 523$ ), and passing outwards and downwards between the cerebrum and cerebellum to reach the ventral surface of the brain: their origin will be best seen at a later stage.

490. The trigeminal nerves (fifth pair, $V$ ), arising from the postero-external region of the pons : each consists of two roots, a large external sensory, and a small internal motor root.

49I. The small abducent nerves (sixth pair, $V I$ ), 
arising from the external edges of the anterior pyramids of the medulla, just posterior to the pons.

492. The facial nerves, or portiones duræ (seventh pair, VII), arise from the lateral regions of the ventral surface of the medulla, just posterior to the corresponding nerves of the fifth pair; their fibres pass directly outwards.

493. The auditory nerves, or portiones molles (eighth pair, VIII), arise close behind and slightly external to the facials; each divides immediately into two large trunks, which pass outwards, parallel with the corresponding seventh nerve.

494. The glossopharyngeal nerves (ninth pair, $I X$ ), vagi or pneumogastric nerves (tenth pair, $X$ ), and accessory nerves (eleventh pair, $X I$ ), arise, in the order mentioned, from the lateral regions of the medulla, just posterior to the cerebellum, and so close to one another that they may easily be mistaken for a single pair. If a sufficient length of the spinal cord has been removed along with the brain, the accessory will be seen to be formed by a slender nerve which arises from the spinal cord slightly posterior to the fifth spinal nerve, and passes forwards, receiving fibres from the cord as it goes, between the dorsal and ventral roots of the first five spinal nerves.

495. The hypoglossal nerves (twelfth pair, XII) arise from the external edges of the anterior pyramids of the medulla, slightly posterior to the three preceding nerves; each is formed by the union of several strands, which closely resemble those of the ventral root of a spinal nerve.

LVII. Place the brain with its dorsal side upwards, and carefully remove successive slices from one of the hemispheres parallel to its upper surface, until the upper surface of the corpus callosum is reached (be careful not to go beyond this point, or some 


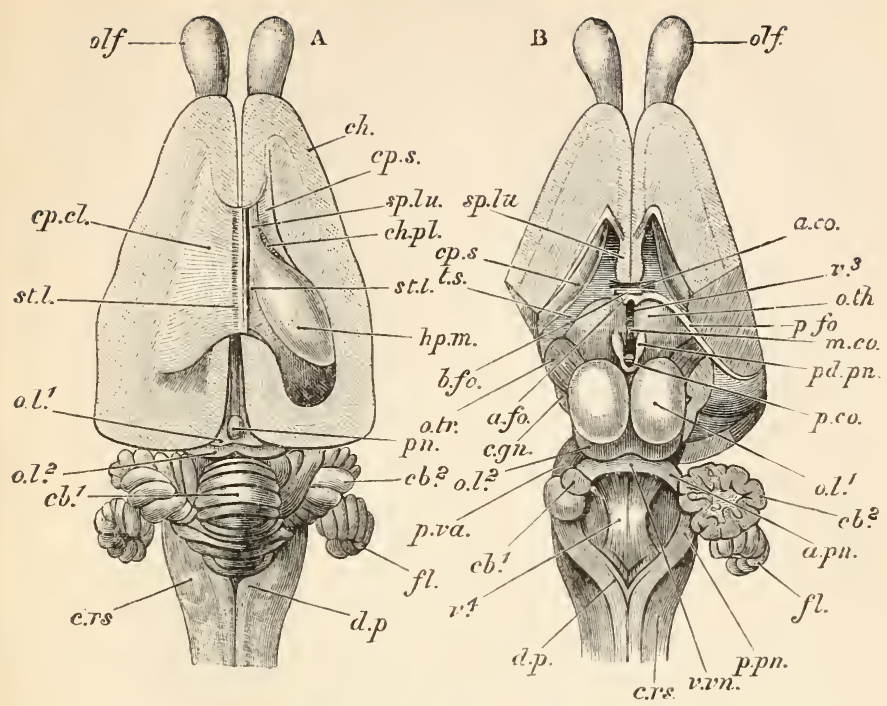

FIG. 71.-Lepus cuniculus. Two dissestions of the brain, from above (nat. size).

In A the left cerebral hemisphere is dissected down to the level of the corpu; callosum : on the right side the lateral ventric!e is exposed. In $\mathrm{B}$ the cerebral hemispheres are dissected down to a little below the level of the anterior genu of the corpus callosum: only the frontal lobe of the left hemisphere $\mathrm{i}$; retained, of the right a portion of the temporal lobe also is left : the velun interpositum and pineal body are removed, as well as the greater part of the hody of the fornix, and the whole of its left posterior pillar: the cerebellum is removed with the exception of a part of its right lateral lobe.

$a . c o$, anterior commissure : $a . f o$, anterior pillar of fornix : $a . p n$, anterior peduncles of cerebellum : b.fo, body of fornix : $c b^{1}$, superior vermis of cerelellum : $c b^{2}$, its lateral lobe: $c \cdot g n$, corpus geniculatum : $c \cdot h$, cerebral hemisphere: $c h . p l$, choroid plexus : $c p . c l$, corpus callosum : cp.s, corpus striatum : c.rs, corpus restiforme : d.p, dorsal pyramid : $f$, flscculus: $h p, m$, hippocampus major: $m . c o$, middle commissure : $o . l^{2}$, nates : $o . l^{2}$. testes : $0 . t h$, optic thalamus: o.tr, optic tract: p.co, posterior commissure : $p \cdot f o$, posterior pillar of fornix : $p n$, pineal body: $p d . p n$, peduncle of pineal body : $p . p n$, posterior peduncles of cerebellum: $p . v a$, fibres of pons Varolii forming middle peduncles of cerebellum : sp.lu, septum lucidum : st.l, stria longitudinalis : t.s, tænia semicircularis: $v . v n$, valve of Vieussens: $v .3$, third ventricle: $v .4$, fourth ventricle. 
of the structures in the lateral ventricle, $\S 498$, may be injured). Note:

496. The central mass of white matter in each homisphere, and the superficial grey matter.

497. The fibres of the corpus callosum, passing horizontally outwards on each side, those in front curving slightly forwards, those behind slightly backwards, to enter the substance of the hemispheres (Fig. 7 I, A, cp.cl). Near the middle line the surface of the corpus callosum is marked by two fine white longitudinal lines, the striæ longitudinales $(s t . l)$.

LVIII. In laying bare the corpus callosum, a cavity, the lateral ventricle $(\S 498)$ will probably have been cut into: if not, carefully scrape away the brain substance near the centre of each hemisphere, about a quarter of an inch external to the posterior boundary of the corpus callosum, until the cavity is met with: lift up the edges of the aperture, and with fine scissors cut away the roof of the lateral ventricle, including the corpus callosum, so as to expose the whole cavity on the one side, to within one-sixteenth of an inch of the middle line of the corpus callosum. Make out:

498. The lateral ventricles (Fig. $7 \mathrm{I}, \mathrm{A}$, right side), or cavities of the cerebral hemispheres, each of which is widest in the middle (body of the lateral ventricle), tapers off almost to a point anteriorly (anterior cornu), and ends behind somewhat bluntly.

499. The hippocampus major (hp.m), a large white eminence, of nearly semicircular section, forming the floor of the body of the lateral ventricle, and extending outwards and backwards : internally it passes into an area, the lyra, by which it is united with its fellow of the opposite side. 
500. The septum lucidum $(s p . l u)$, a somewhat triangular body, formed externally of grey matter, lying beneath the anterior end of the corpus callosum and immediately in front of the anterior end of the hippocampus: it forms the inner wall of the anterior cornu of the lateral ventricle.

501. The choroid plexus (ch.pl), an extremely vascular membrane lying in a depression just in front of the hippocampus major, and passing beneath the corpus callosum to join its fellow of the opposite side through a transverse passage, which puts the third ventricle $\left(\S_{5} \mathrm{r} 3\right)$ in communication with the lateral ventricles; this passage is the foramen of Monro. The connection of the two choroid plexuses through the foramen of Monro is best seen by exerting a slight traction on one of them.

502. The corpus striatum (cp.s), an eminence of grey matter forming the floor of the anterior cornu of the lateral ventricle, and separated from the septum lucidum and hippocampus major by the depression in which the choroid plexus lies.

LIX. Dissect the other hemisphere in the same way, observing the same points again, and noting further :

503. The connection of the corpus callosum with the underlying parts of the brain, namely with the lyra behind and with the septum lucidum in front.

504. By dividing the corpus callosum transversely, carefully separating its two ends from the underlying parts and reflecting them, it is seen to bend slightly downwards at its anterior end, forming the anterior genu, while at its posterior end it bends sharply downwards and then forwards, forming the posterior genu.

505. By the removal of the corpus callosum the septum lucidum is found to consist of two layers, between which is 
included a space answering to the fifth ventricle of the higher mammalia, but evidently a portion of the space between the right and left hemispheres. ${ }^{1}$

LX. Trace the course of the hippocampus major by carefully cutting away the outer part of the temporal lobe : note :

506. The descending cornu of the lateral ventricle, exposed by the removal of the outer wall of the temporal lobe; it passes first almost directly outwards, then downwards, and finally inwards and forwards. The hippocampus major is continued the whole distance along its inner wall.

507. The posterior pillar of the fornix (Fig. $7 \mathrm{I}, \mathrm{B}$, p.fo), a flat white band forming the projecting anterior edge of the hippocampus major, and continued with the latter into the descending cornu. Anteriorly it becomes con. nected with its fellow of the opposite side just behind the septum lucidum, forming the body of the fornix.

508. The tænia semicircularis $(t . s)$, a thin band of white matter, just in front of the posterior pillar of the fornix, and forming a sort of bed for the choroid plexus.

509. The fissure of Bichât, a slit-like space between the tænia semicircularis and the posterior pillar of the fornix; it leads on to the under surface of the brain, between the anterior and inner edge of the temporal lobe and the optic tract.

I.XI. Make a transverse incision through the body of the fornix, immediately over the foramen of

1 In the higher mammalia, e.g. man, the septum lucidum becomes very thin and translucent, whence its name, and, the anterior genu of the corpus callosum being very well developed, and turning round the anterior edge of the septum lucidum, the latter takes on the form of a double membrane, situated between the corpus callosum above and in front, and the body of the fornix ( $\$ 507)$ behind. 
Monro, cutting down to the latter: from the middle of this cut take a longitudinal incision backwards through the lyra: press aside the posterior portion of the hemispheres, now completely separated from one another, and make out :

5 10. The hippocampal sulcus, a deep, curved groove on the inner face of the temporal lobe, corresponding with the elevation of the hippocampus major.

5Ir. The velum interpositum, a delicate vascular membrane, continued backwards from the conjoined choroid plexuses, and forming a roof to the third ventricle $\left(\S 5^{\mathrm{I}} 3\right)$.

5 I2. The optic thalami (o.th), large masses of mixed grey and white matter, separated in front from the corpora striata by the depression in which the tænia semicircularis lies, and covered dorsally by the velum interpositum.

5I3. By removing the velum interpositum the third ventricle $(v \cdot 3)$ is exposed; it is a median vertical fissure, with which the lateral ventricles communicate, anteriorly, by the foramina of Monro.

5I4. The anterior commissure (a.co), a narrow band of white fibres, passing transversely, immediately in front of the third ventricle, between the corpora striata.

5I5. The anterior pillars of the fornix $(a . f o)$, a pair of delicate, vertical, white bands (cut short by the severance of the body of the fornix), which pass directly downwards immediately posterior to the anterior commissure.

516. The middle or soft commissure (n.co), a wide mass of grey matter, passing between the optic thalami nearly level with their dorsal surface, and, in an upper view, almost obliterating the cavity of the third ventricle.

5 r7. On the upper surface of the hinder half of each 
optic thalamus is a thin white band, the peduncle of the pineal body $(p \cdot p n)$ : the two peduncles join with one another at the posterior boundary of the thalami, and are continued into a flat band about a quarter of an inch long, which ends at the posterior boundary of the cerebral hemispheres in a rounded vascular mass: this is the pineal body: it rests on the dorsal surface of the optic lobes.

518. The posterior commissure (p.co), seen by turning aside the stalk of the pineal gland : it is a delicate transverse band of white fibres, somewhat smaller than the anterior commissure, connecting the thalami with one another just below the junction of the peduncles of the pineal body.

519. The optic lobes, or corpora quadrigemina $(o . l)$, two pairs of rounded elevations, composed externally of grey matter, and lying immediately above the crura cerebri, and bounded by the optic thalami in front and by the cerebellum behind. The anterior larger pair are regularly ovoidal in shape, and are called the nates $\left(0 . l^{1}\right)$ : the posterior pair, or testes $\left(0 . l^{2}\right)$, are transversely elongated, and lie at a lower level than the nates.

520 . By removing the remainder of the temporal lobe of one side, the optic tracts $(0 . t r, \S 486)$ are seen to curve round the antero-external corner of each optic thalamus, from the chiasma, and then to pass backwards and inwards to the nates.

52 I. The corpus geniculatum $(c . g / n)$, a small rounded elevation on the external surface of the optic thalamus, immediately posterior to the optic tract.

LXII. Cut away the whole of the central part of the cerebellun, leaving only its points of attachment, at the sides, to the medulla oblongata. Note:

22. The fourth ventricle $(i \cdot 4)$, a flat, lozenge-shaped 
cavity, bounded laterally by the dorsal pyramids of the medulla, and roofed in by the cerebellum and the pia mater. Its floor is marked by a median groove, ending posteriorly in a pointed depression, the calamus scriptorius, which leads into the central canal of the spinal cord.

523. The valve of Vieussens ( $7.0 n)$, a thin transverse band, forming a sort of ledge over the anterior end of the fourth ventricle, and connected by its anterior edge with the testes; the fourth pair of nerves spring from its upper surface: beneath it is a small aperture leading into the aqueduct of Sylvius ( $\$ 526)$.

524. The anterior $(a . p n)$, middle, and posterior $(p . p n)$ peduncles of the cerebellum : the anterior connect it with the testes, and sc bound the valve of Vieussens laterally; the middle are continuous on each side with the pons Varolii; and the posterior connect it to the dorsal side of the medulla oblongata, and are the anterior ends of the restiform bodies.

525. The arbor vitæ, or tree-like appearance produced in a section of the cerebellum by the intermixture of its grey and white matter.

LXIII. Pass a guarded bristle from the fourth ventricle through the aperture beneath the valve of Vieussens $(\$ 523)$, and cut down upon it by removing the optic lobes, so as to expose :

526. The aqueduct of Sylvius (Fig. 72), or iter a tertio ad quartum ventriculum, a narrow passage by which the third and fourth ventricles are put in communication with one another. It is bounded above by the optic lobes, below by the crura cerebri.

527. Note at the same time the absence of optic ventricles or cavities in the optic lobes. 
LXIV. Cut tnrough the three commissures of the third ventricle, and press the thalami gently apart. Note :

528. The infundibulum (inf), a funnel-shaped cavity, into which the third ventricle is continued below, and which, after the removal of the pituitary body, opens by the small aperture already noticed on the base of the brain (see $\S 485$ ).

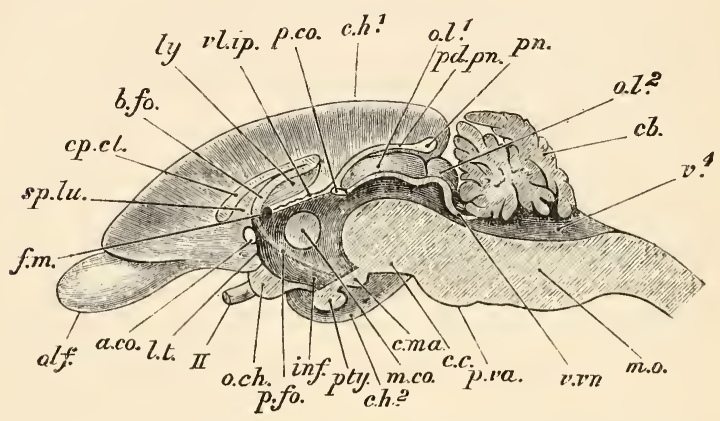

FIG. 72.-Lepus cuniculus. Longitudinal vertical section of the brain (nat. size).

$a . c o$, anterior commissure : p.fo, anterior pillar of fornix, supposed to be seen through optic thalamus : $b$. fo, body of fornix : $c b$, cerebellum, showing arbor vitæ : c.c, crus cerebri : c. $h^{1}$, cerebral hemisphere: $c . h^{2}$, its temporal lobe : c.ma, corpus mammillare : cp.cl, corpus callosum : $f . m$, foramen of Monro: inf, infundibulum : ly, lyra: m.co, middle commissure : $m .0$, medulla oblongata : o.ch, optic chiasma : $0 . l^{1}$, natis : $o . l^{2}$, testis : olf, olfactory lobe : p.co, posterior commissure : $p n$, pineal body : $p d . p n$, its peduncle : $p t y$, pituitary body : $s p . l u$, septum lucidum : $v l . i p$, velum interpositum: $v \cdot v n$, valve of Vieussens: $v \cdot 4$, fourth ventricle: $I I$, optic nerve.

529. The lamina terminalis, or lamina cinerea (l.t), the thin vertical anterior wall of the third ventricle.

530. By carefully scraping away the inner surface of one of the optic thalami, trace the anterior pillar of the fornix ( $p$. fo), which, after curving over the foramen of 
Monro ( $\S 501,5 \circ 7,5^{1} 3$ ), passes downwards and backwards, and ends in the corpus mammillare: it is best made out in a fresh brain.

LXV. In another brain make a longitudinal vertical section of the whole organ, a very little to one side-say the left - of the median line. Make out, without further dissection, the relation of the median ventricles, infundibulum, corpus callosum, commissures of the third ventricle, \&c. .

LXVI. In a third thoroughly hardened brain make a series of transverse sections, and note in them the relations of the parts already seen by dissection. The sections should be taken through-

(a). The anterior end of the corpus callosum and the septum lucidum.

(b). The anterior commissure.

(c). The foramen of Monro and optic chiasma.

(d). The middle commissure.

(e). The posterior commissure and infundibulum.

$(f)$. The nates and corpus mammillare.

$(g)$. The testes, valve of Vieussens, and pons Varolii.

(h). The flocculi and hinder part of the medulla oblongata. 



\section{WORKS OF REFERENCE.}

The following list makes no pretence to be a complete bibliography, but simply aims at giving the names of a few books and papers which students may consult with advantage.

The works marked with an asterisk were published after the corresponding sections of this book were written.

I. Brovn, H. G. Die Klassen und Ordnungen des Thier-reichs: VI. Band (Vertebrata). Leipzig and IIeidelberg, 1873 and onwards.

The volume on the Amphibia is the only cne completed, the others are still coming out in parts.

2. Bruhi, C. B. Zootomie aller Thier Klassen. Vienna, IS75 anci onwards.

Incomplete : contains many good figures illustrating vertebrate anatomy.

3. Chauveau, A. L'Anatomie comparée des Animaux domestiques. Paris, $\mathrm{ISS}_{3}$ (New Edition).

Contains a gord deal of information on the Anatomy of the Rabtit (omitted in the English edition.)

4. Ewart, J. C. On Vaccular Peribranctial Spaces in the Lamprey. Journal of Anatomy and Physiology, Vol. xii., IS78, p. 232.

5. Ewart, J. C. Notes on the Abdominal Pores and Urogenital Sinus of the Lamprey. Ib., Vol. x., IS76, p. 488 .

6. Gegenbaur, C. Das Kopfskelet der Selachia. Leipzig, 1872 .

7. Gegenbaur, C. Untersuch. zur vergleich. Anatomie der Wirbelthieze, I Heft, Carpus und Tarsus. Leipzig, IS64.

8. Giacomini. Method of Preparing the Brain, described in the Journ. of Anat. and Phys. Vol. xiii., 1S79, p. 282. 
9. Günther, A. An Introduction to the Study of Fishes. Elinburgh, iSSO.

10. Haswell, W. A. Notes on the Anatomy of Birds. Proceeding; of the Linnean Society of New South Wales. Vol. iv., I $879, \mathrm{p} \cdot 303$.

I1. Haswell, W. A. Some Points in the Myology of the Common Pigeon. Journ. of Anat. and Phys. Tol. xvii., ISS 3 , p. 218.

12. *Howes, G. B. The Presence of a Tympanum in the Genus Raia. Journ, of Anat. and l'hys. Vol. xvii., ISS3, p. ISS.

13. IIUxley, T. H. A Manual of the Anatomy of Vertebrated Animals. London, IS7I.

Contains a short account of the anatomy of the Rabbit.

14. Huxley, T. H. The Nature of the Craniofacial Apparatus of Petromyzon. Journal of Anatomy and Physiology, Vol. $x_{\text {., }}$ I876, p. 412.

I5. Huxley, T. H. On the Respiratory Organs of Apteryx. Pr.s. Zosl. Sxc., ISS2, p. 560.

Contains an account of the general disposition of these organs in birds.

16. Hyrtl, J. Das arterielle Gefäss system der Rochen. Wien. Sitz. Der. xxv., I857, and Wien. Denkschr. xv., 1858 .

17. Jourdain, S. Sur la Veine poste rénale. Annales des Sciences Naturelles, Zool., Sér. iv., tom. xii., I $\$ 59$.

IS. Klein, E. The Organ of Jacobson in the Rabbit. Quart. Journ. of Micros. Science, Vol, xxi., ISS I, p. 549.

19. Krause, W. Die Anatomie des Kaninchens. Leipzig, IS68.

20. Milne-Edwards, H. Leçons sur la Physiologie et l'Anatonie comparée. Paris, $\mathrm{I}_{57}$-79.

21. Müller, Johannes. Tergleich. Anatomie der Myxinoiden. Berlin, I840.

22. Monro, A. The Structure and Physiology of Fishes. Edinburgh, 1785 .

Contains several excellent plates illustrating the anatomy of the Skate.

23. Owen, R. On the Anat omy of Vertebrata. London, I866I $\$ 68$.

24. Parker, T. J. On the Intestinal Spiral Valve in the Genus Raia. Transactions of the Zoological Society, Vol. xi., ISSo, p. 49.

25. Parker, T. J. On the Venous System of the Skate. Transactions of the N. Z. Institute, Vol, xiii., ISSo, p. 413. 
26. Parker, T. J. On a New Method of preparing Cartilaginous Skeletons and other Soft Animal Structures. Ib., Vol. xiv., p. 258.

27. *PARKER, IV. K. On the Structure and Development of the Skull in Marsipobranch Fishes. Phil. Trans., IS8 3 , p. 40.

28. PARKER, W. K. On the Structure and Development of the Skull of Sharks and Skates. Trans. Zosl. Soc., Vol. x., IS 7 S, p. 189.

29. PARKer, WV. K. On the Structure and Development of the Skull in the Lacertilia. Phil. Trans., Part 2, IS79, p. 595.

3o. Parker, IV. K., and G. T. Bettaiy. The Morphology of the Skull. London, 1871 .

3I. PARker, W. N. Note on some Points in the Anatomy of the Cæcum in the Kabbit and Hare. Proc. Zool. Soc., IS81, p. 624.

32. Rollestox, G. Forms of Animal Life. Oxford, IS7o.

Includes a description of the vertebral column of the Rabbit, and of both the skeleton and s ff parts of the Pigeon.

33. Stannius, H. Handbuch der Anatomie der Wirbelthiere. Berlin, IS54.

34. * Wiedershim, R. Lehrbuch der vergleichenclen Anatomie der IVirbelthiere. Jena, ${ } S_{3}$. 

INDEX。

C C 



\section{INDEX.}

A.

AbDominal pore:-Lamprej, I6; Skate, 43.

Acetabulum, see Hip-girdle.

Acromion, see Shoulder-girdle.

Adrenal or supra-renal capsule:-Skate, 60 ; Pigeon, 23I ; Rabbit, 300, 302.

Air-bladder:-Cod, Iog, 115.

Air-sacs:-Pigeon, 216, 218, 219, 243, 246. inflation of, $21_{4}$. injection of, 214 , note.

Almentary canal:-Lamprey, $x_{4}, 18$; Skate, 47; Cod, IIr; Lizard, 163; Pigeon. 226 ; Rabbit, 304.

Ala spuria:-Pigeon, 212.

Alisphenoid :-Cod, 94; Pigeon, I9r; Rabbit, 270.

Angular bone, see Mandible.

Anterior vertebral plate:-Skate, 29.

Anticl.nal vertebra :-Rabbit, 265 .

Antorbital cartilage :-Skate, 37 .

Anus, see External characters.

Aorta, arch of:-Lizard, 172; Pigeon, 238, 240, 243; Rabbit, 311, 327.

dorsal:-Lamprey, 16, 2x; Skate, 60, 67; Cod, I15; Lizard, 164, з73; Pigeon, 234; Rabbit, 299, 306, 3I I, 327.

ventral:-Lamprey, 2I; Skate, 6r ; Cod, II 6.

Appendix vermiformis:-Rabbit, 298, 305. Apteria or Featherless spaces:-Pigeon, $2 \mathrm{Ir}$.

A fueduct of Sylvius :-Lamprey, 23; Skate, 73; Cod, 129; Lizard, 180; Pigeon, 26o; Rabbit, 377.

Aqueductus vestibuli :-Skate, 35, 8r.

Aqueous humour, see Eye.

Arachnoid fluid:-Skate, 46 ; Cod, 110.
Arbor vitæ of cerebellum:-Pigcon, 26r ; Rabbit, 377.

Artery-

basilar :-Rabbit, 365 .

brachial:-Skate, 67; Lizard, 174; Pigeon, 237; Rabbit, 312.

branchial (atferent and efferent):Lamprey, 21, 22; Skate, 61, 66, 69; Cod, II8, I21.

coronary:-Skate, 6r ; Rabbit, 308, 327 .

carotid (common. external, and interral):-Skate, 68 ; Cod, 121 ; Lizard, I73: Pigeon, 237, 239: Rabbit, 312. caudal:-Lamprey, 17; Skate, 67; Pigeon, 235.

cerebral (anterior, middle, and posterior):-Rabbit, 365 .

cœliac:-Skate, 50,67 ; Cod, II 3 ; Lizard, 164; Pigeon, 225, 235; Rabbit, 299.

cœliaco-mesenteric:-Lizard, 164.

duodenal:-Skate, 49; Lizard, 164; Rabbit, 303 .

epibranchial:-Cod, xrg.

epigastric:-Rabbit, 315 .

fem ral :-Lizard, 170; Pigeon, 234 ; Rabbit, 315 .

gastric:-Skate, 50; Pigeon, 225; Rabbit, 303.

gastro-duodenal :-Pigeon, 225.

gastro-hepatic:-Skate, 50; Lizard, I64.

hepatic:-Skate, 50; Lizard, 163; Rabbit, 303.

hyoidean:-Skate, 68; Cod, $12 x$.

hypobranchial :-Skate, $6 \mathbf{r}$.

iliac (common, external, and internal): -Pigeon, 235: Rabbit, 3II, 315.

ilio-lumbar: -Kabbit, 315.

C $\mathrm{C} 2$ 
Artery (continused) -

innominate:-Skate, 6r ; Pigeon, 237 : Rabbit, 312 .

lumbar:-Rabbit, 3 I 4 .

mammary :-Pigeon, 238; Rabbit, 314. mesenteric (anterior or superior, and posterior or inferior):-Skate, 49, 51, 67; Cod, Ir3; Lizard, 165; Pigeon, 226, 235 ; Rabbit, 299, 300. ophthalmic:-Cod, $\mathrm{r} 75$.

pecioral:-Pigeon, 237 .

phrenic :-Rabbit, 3i4.

pulmonary:- Lizard, I72, I73: Pigeon, 238, 240, 243; Rabbit, 308, 325,328 .

renal:-Skate, 67; Pigcon, 235 ;

Rabbit, 314 .

sacral, median:- $R$ atbit, ${ }^{\mathrm{r}} 4$.

sciatic:-Pigeon, 234 .

spermatic:-Skate, 67; Rabbit, 314.

splenic: $-R$ abbit, 303 .

subclavian:-Skate, 67 ; Lizard, 174 ; Pigecn, 237 ; Rabbit, 312, 314.

thoracic:-Rabbit, 314 .

vertebral:-Skate, 68; Pigeon, 239: Rabbit, $3 \mathbf{1 4}$.

Articular bone, sce Mandible.

Articulations-

elbow:-Rabbit, 35r.

knee:-Rabbit, $36+$.

shoulder:-Rabtit, 35 r.

thigh: - Rabbit, 364 .

vertebræ:-Skate. 32, 33 : Cod, 88 ;

Lizard, т32; Pigeon, 186; Rabbit, 267.

Arytenoid cartilage, see Larynx.

Atlas vertebra:-Cod, 89; Lizard, I33: Pigeon, 186; Rabbit, 267.

Auditory capsule:-Lamprey, 5; Skate, 34; Cod, 93; Lizard, 136; Pigeon, 193; Rabbit (periotic), 272.

meatus, external:- - kabbit, 331 . internal, see znder Foramen.

organ:-Lamprey, 25; Skate, 80 ; Cod, 122; Pigeon, r93.

ossicles :-Rabbit, 277.

Auricle, sce Heart.

Axis vertebra:-Lizard, 133; Pigeon, 185; Rabbit, 267.

\section{B.}

BARBULE:-Cod, IO2.

Basal cartilage of fins:- Skate, 33 .

Basale metapterygii or basipterygium :Skate, 40.

Basi-branchial, see Branchial arches.

Basi-hyal, sce $\mathrm{H}$ ycid.

Basi-occipital:-Cod, 91 ; Lizard, 140; Pigeon, 190, 192; Rabbit, 269.

Basi-pterygoid processes:-Lizard, I4I ; Pigeon, ${ }^{2}$.
Basi-sphenoid:-Lizard, I4r ; Pigeon, I92; Rabbit, 270.

Basi-temporal:-Pigcon, 192.

Bile ducts (hepatic, cystic, and common bile ducts):-Lamprey, 16; Skate, 49, 50; Cod, 112, 113: Lizard, :65, 170; Pigeon, 223, 224 ; Rabbit, 303.

Bladder, gall:-Skute, 49; Cod, 108 : Lizard, 161 ; Rabbit, 296.

urinary:-Skate, 58 ; Cod, 109 ; Lizard, 168, 171; Rabbit, 292, 320, 323.

Body cavity, see Cœl me.

Brachial ossicles:-Cod, ror.

plezus, see under Nerve plexus.

Lrain, removal of:-Lamprey, 23; Skate, 46; Cod, I ro; J,izard, I76; Pigeon, 221 ; Rabbit, 294.

dissection of:-Lamprey, 23; Skate, 7o; Cod, 123; Lizard, 176; Pigeon, 254 ; Rabbit, 365 .

Branchial apertures, external, see External characters.

internal, see Mouth cavity.

arches:-Skate $38,66,68 ; \operatorname{Cod}, 9^{3}$, 121 ; Lizard, 146; Piyreon, 198; Rabbit, 276 .

basket:-Lamprey, 7 .

filaments, see Gills.

rays: - Skate, 69 .

Branchiostegal membrane:-Cod, 103. rays: $-\operatorname{Cod}, 98$

Broad ligament:-Lizard, I68; Rabbit, 322.

Bronchi:-Lizard, 175; Pigeon, 220, 244, 245: Rabbit, 308, 325 .

Bulbus arteriosus, see Heart.

Bulla tympani:-Rabbit, 272.

Bursa Fabricii :-Pigeon, 235, 236.

\section{C.}

Cecun coli:-Lizard, r64 ; Pigeon, 223; Rabbit, 292, 304, 306.

nasal :-Lamprey, 25.

oral:-Lamprey, 20 .

Calamus scriptorius:-Skate, 72; Rabbit, 377 .

Campanula Halleri:-Cod, r22.

Capitular facet, see Vertebra.

Carpus, see Manus, skeleton of.

Cartilages of skull:-Lamprey, 6, 7 .

Centrum, see Vertebra.

Cerato-branchial, see Branchial arches.

Cerato-hyal, see Hyoid.

Cere:-Pigeon, 209.

Cerebellum:-Lamprey, 23; State, 70: Cod, 124; Lizard, 178; Pigeon, 256, 258 ; Rabbit, 366 .

peduncles of:-Rabbit, 367,377 .

Cerebral hemispheres:-Lamprey, 24; Cod, 124: Lizard, 178; Pigeon, 256 ; Rabbit, 368 . 
Circle of Willis : - Rabbit. 365 .

Circulus cephalicus:-Cod, $\mathrm{r} 2 \mathrm{r}$.

Chevron bones:-Lizard, I34.

Chondrocranium :-Lizard, I43.

Ch rda tympani :-Skate, 80.

Choroid, see Eye.

processes, sce Eye.

gland:-Cod, 122.

plexus:-Lizard, 180; Pigeon, 259; Rabbit, 373 .

Claspers:-Skate, 41.

gland of, 60 .

skeleton of, 40 .

Clavicle, see Shoulder-girdle.

Cloaca:-Skate, 44, 47; Lizard, 168, 170 ; Pigeon, 223, 235.

Colome (including abdominal and thoracic cavities):-Lamprey, 13; Skate, 43; Cod, 107; Lizard, 159; Pigcon, 216; Rabbit, 231, 293.

Colon:-Skate, 47; Rabbit, 292, 324.

Columella or Epipterygoid:-Licard, I4 2.

auris:-Lizard, I37, I42; Pigeon, I95, $25 \mathrm{I}$.

Commissures of brain (anterior, middle, and fosterior):-Lizard, I8I ; Pigeon, $26 a$; Rabbit, 375,376 .

Conus arteriosus, sée Heart.

Coracoid, sce Shoulder-girdle.

Cornea, see Eye.

Coronary bone, see Mandible. ligament:-Cod, I12; liublit, 296.

Corpus or Corpora-

adiposa:-Lizard, гб.

bigemina, see $\mathrm{O}$ ptic lubes.

callosum:-Rabbit, 368, 372, 373.

cavernosa, see Penis and clitoris.

geniculatum :-Rabbit, 376 .

mammillare :-Rabbit, 360 .

quadrigemina, see Optic lobes.

restiformia, see Medulla oblcngata.

spongiosum, see Penis and clitoris.

striata:-Liznrd, 180; Pigeon, 258; Rabbit, 373 .

Cowper's glands:- Rabbit, 320,323 .

Cranial fossæ, see under Fossæ.

Cribriform plate:-Rabbit, 273.

Crop:-Pigeon, 214, 2 I5.

Crura cerebri:-Skate, 72; Lisard, I78; Pigeon, 256 ; Rabbit, 368.

Crystalline lens, see Eye.

Cystic duct, see Bile duct.

\section{D.}

DEMIERANCHIA or Half-gill:-Lamprey, I8; Skate, 46.

Dentary, see Mandible.

Diaphragm :-Rabbit, 292, 306.

Ductus arteriosus:-Rabbit, 3II. Cuvierii, see Vein, precaval.
Duodenum:-Skate, 47; Lizard, 164; Pigeon, 220, 226; Rabbit, 297, 304 .

Dura mater:-Skate, 46 ; Rabbit, 295.

E.

Epencephalon, sec Cerebellum.

Epididymis:-Skate, 56; Lizard, 165 ; Ralbit, 318 .

Epibranchial, see Eranchial arches.

Epigluttis:-Rabbit, 333 .

Epihyal, see Hyoid.

Epiotic:-Cod, 93; Lizard, 140; Pigeon, I94.

Epiphyses of vertebræ: - Rabbit, 264 .

Epipterygoid or Columella :-Lizard', 142. Ethmo-turbinal, see Turbinal.

Eustachian tube cr recess:-Lizard, 176; Pigeon, 192, 250; Rabbit, 272, 332, 336.

valve, see Heart, dissecticn of.

Eye, see Exiernal characters.

dissection of:-Skate, 84 ; Cod, 122; Pigeon, 250 ; Ralbit, 330.

Eyelids, see External characters.

Exoccipital:-Cod, 91 ; Lizard, 140 ; Pigeon, 19o; Rabbit, 269 .

External characters:-Lamprey, 8; Skatc, 40; Cod, ror; T.isard, 153; Pigcon, 209 ; Rabbit, 286.

F.

FAlCiform ligament:-Pigeon, 219, 222.

Fallcpian tube:-Skate, 57 ; Rabbit, 322, 324 .

Falx cerebri:-Rabbit, 295 .

Fascia, dor:o-lumbar:-Rabbit, 339. lata:-Rabbit, 354.

Feathers, structure of:-Pigeon, 213. arrangement of:-Pigeon, 211 .

Femoro-tibial or semilunar cartilages:Lizard, $152 ;$ Rabbit, 285 .

Femur:-Lizard, 15 I; Pigeon, 206; Ratbit, 283 .

Fenestra ovalis and rotunda:-Lizard, I42; Pigeon, 195; Rabbit, 272.

Fibula:-Lizard, 152 ; Pigeon, 208 ; Rabbit, 285 .

Filoplume:-Pigeon, 2c9, 214.

Fins, sce External characters. skeleton of (fin-rays, \&c.):-Lamprey, 4 ; Skate, 33, 39. 40; Cod, 88, 101.

Fissure of Bichât:-Rabbit, 374 .

Fontanelles, cranial :-Skate, 36 ; Lizard, 138.

Foramen cordiforme:-Lizard, I50. magnum:-Skate, 34 ; Cod, 91 ; Lizard, 136; Pigeon, 190; Rabbit, 268. 
Foramen (continued) -

of Monro:-Lizard, 180, 181 ; Pigeon. 259 ; Rabbit, 373 .

obturator, see Hip-girdle.

olfactory:-Skate, 3o; Lizard, I44 : Pigeon, 191 ; Rabbit, 273.

optic:-Skate, 30 ; Pigeon, 192; Rabbit, 27 I.

for 3 rd nerve:-Skate, 36 .

for 4 th nerve:-Skate, 36 .

for $3^{1 \mathrm{~d}}$, $4^{\text {th }}$, first division of $5^{\text {th }}$ and 6th nerves (sphenoidal fissure):Rabbit, 27r.

for 3 rd division of $5^{\text {th }}$ nerve (foramen lacerum):-Rabbit, 273, 276 .

for $5^{\text {th }}$ nerve:-Pigeon, 194 .

for 5 th and 7 th nerves:-Skate, 35; Cod, 93; Lizard, 143 .

for 7 th nerve:-Pigeon, I94; Rabbit, 272.

for 8th nerve (internal auditory mea. tus):-Lamprey, 5; Skate, 36; Pigeon, 194 ; Rabbit, 272.

for 9 th and Ioth nerves:-Skate, 35 ; Cod, 9r.

for 9 th, roth, and irth nerves (foramen lacerum posterius):-Lizard, I42 ; Pigeon, r94; Rabbit, 273.

for I th nerve (condyloid foramen):Pigeon, 194; Rabbit, 269.

Fore limb, see External characters.

skeleton of:-Skate, 39; Cod, ror : Lizard, 147; Pigeon, 201; Rabbit, 280.

Fornix :-Rabbit, 374, 375, 378.

of Gottsche:-Cod, 128 .

Fossa ovalis, see Heart, dissection of.

Fossæ of skull :-Lizard, $\mathrm{1}_{3} 6, \mathrm{I}_{37}$; Pigeon, 193; Rabbit, 268, 276.

Frontal bone:-Cod, 94; Lizard, 138 ; Pigeon, 190; Rabbit, 270.

Fronto-nasal process:-Skate, 42.

Furcula:-Pigeon, 20r.

\section{G.}

Gall-BLADDER, see under Bladder.

Ganglion, cœliac:-Rabbit, 299.

sympathetic:-Skate, 65; Rabbit, 3 I 2.

Genital aperture, see External characters.

Gills:-Lamprey, I 7 ;kate, 45, 60, 69; Cod, 103, 120, 121.

Gill-rakers :-Cod, 120.

Gizzard:-Pigeon, 220, 227.

Gleno:d cavity, facet, or surface, see Shoulder-girdle, Squamosal, and Quadrate.

Glutt $\mathrm{S}$, see Mouth cavity.

Gubernaculım:-Rabbit, 318 .

Gullet:-Lamprey', 16, 18, 20; Skate, 47: Cod, rog, I11 ; Lizard, I75; Pigeon, 215, 217, 243; Rabbit, 296, 306, 311.
H.

Hemal arch, process, and spine, see Vertebra.

Half-gill, see Demibranchia.

Hallux, see Pes.

Hard palate:-Lamprey, 4.

Harderian gland:-Pigeon, 249 ; Rabbit, 330.

Head-kidney (so-called):-Cod, Ir5.

Heart:-Lamprey, 20; Skate, 45; Cod, I10; Lizard, 162, I71 ; Pigeon, 220, 237 ; Rabbit, 293, 308.

dissection of:-Lamprey, 22; Skate, $64 ;$ Cod, 118 ; Lizard, 174; Pigeon, 24I; Rabbit, 326.

Hepatic duct, see Bile duct.

Hind limb, see External characters.

skeleton of:-Skate, 40; Cod, ror : Lizard, 150; Pigeon, 205; Rabbit, 283.

Hip-girdle or Pelvis:-Skate, 39: Cod, 101; Lizard, 150 ; Pigeon, 204; Rabbit, 282.

Hippocampal sulcus :- Rabbit. 375 .

Hippocampus major:-Rabbit, 372 .

Humerus:-Lizard, 148; Pigeon, 202; Rabbit, 280 .

Hyoid arch or bone:-Skate, 37: Cod, 97: Lizard, 145; Pigeon, 198; Rabbit, 276.

Hyomandibular:-Skate, 37 ; Cod. 95.

Hypobranchial, see Branchial arches.

Hypohyal, see Hy id arch.

Hypural :-Cod, go.

I.

ILEO-COLIC valve :-Rabbit, 306 .

lleum:-Lizard, I64; Pigeon, 220, 226 ; Rabbit, 297, 304.

Ilium, see Hip-girdle.

Incus:-Rabbit, 277 .

Infundibulum:-Skate, 72 ; Lizard, 178 ; Pigeon, 256; Rabbit, 369, 378.

Injection of vascular system:-Skate (and General Directi ns), 48 ; Cod, IrI, note; Lizard, 162, note; Pigeon, 218, note; Rabbit, 208, note.

Innominate bone, see Hip-girdle.

Integument, see External characters.

Inter-clavicle, see Shoulder-girdle ( $\mathrm{Liz}_{\mathrm{z}}$ ard).

Inter-hyal, see Hyoid (Lizard).

Inter-muscular bones:-Cod, 90 .

Inter-neural plates:-Skate, 32.

Inter-opercular, see Operculum.

Inter-crbital septum:-Lizard, I37, I43; Pigeon, r9i.

Inter-parietal:-Rabbit, 270.

Inter-renal body:-Skate. 59 .

Inter-spinjus bones:-Cod, 90 . 
Inter-vertebral foramina, see Vertebra.

ligament:- Rabbit, 267.

substance:-Skate, 33 .

Intestine :-Lamprey, 14; Skate, 44, 47 : Cod, 107, III; Lizard, 161, 164; Pigeon, 220, 223, 226; Rabbit, 292, 304,305 .

Intra-colic valve:-Rabbit, 306 .

Ischium, see Hip-girdle.

\section{J.}

JACOBSon's organ and cartilage:- $L i z$ ard, 176 ; Rabbit, 273, 336 .

Jejunum:-Pigeon, 226.

Jugal or Malar:-Lizard, 138; Pigeon, 197 ; Rabbit, 274.

K.

Kidneys:-Lamprey, $5_{5}$; Skate, 54 ; Cod. 116; Lizırd, 169; Pigeon, 229: Rabbit, $299,302,314,324$.

\section{L.}

LABIA majora:- Rabbit, $32 \mathrm{r}$.

Labial cartilages:-Skate, 36 .

Lacrymal bone:-Cod, roo; Lizard, 139; Pigeon, 196; Rabbit, 275.

gland:-Pigeon, 249; Rabbit, 330.

Lacteals:-Rabbit, 304.

Lamina perpendicularis:- - Rabbit, 273 . terminalis, Pigeon, 258; Rabbit, $37^{8}$.

Larynx:-Lizard, x75; Pigeon, 248 ; Rabbit, 289,313 .

disseciicn of:-Kabbit, 336 .

Lateral line:-Cod, 102 .

Ligamentum suspensorium:-Pigeon, 186.

Ligaments, see Articulations.

Limbs, see Fore limb and Hind limb.

Linea alba :-Rabbit, $29 \mathrm{r}$.

Liver:-Lamprey, 13; Skate, 44; Cod, I07; Lizard, 159; Pigeon, 220, 223: Rabbit, 292, 296.

Lobi inferiores:-Skate, 72 ; Cod, $\mathbf{x} 24$. posteriores:-Cod, 128 .

Lower jaw, see Mandible.

Lungs:-Lizard. 162, 168, 175; Pigeon, 221, 244-246; Rabbit, 294, 325 .

Lymphatic gland:-Pigeon, 240.

Lymph-sinus, sub-vertebral:- Lamprey, 15.

Lyra:-Rabbit, $37^{2}$.

M.

Malleus:-Rabbit, 277 .

Mammary glands:-Rabbit, 280 .

Mandible or Lower jaw :-Skate, 37 ; Cod, 97: Lizard, 145 ; Pigeon, 197 ; Rabbit, 276 .
Manubrium, see Sternum.

Manus, see External characters. skeleton of:-Lizard, I49: Pigeon, 202 ; Rabbit, $28 \mathrm{I}$.

Maxilla:-Cod, 96; Lizard, r38; Pigeon, I96; Rabbit, 274.

Maxillo-palatine process:-Pigeon, 196.

Meckel's cartilage:-Skate, 37 ; Cod, 97 ; Lizard, 145; Pigeon, 198 .

Mediastinum:-Rabbit, 294, 307 .

Medulla oblongata:-Lamprey, 23 ; Skate, 70; Cod, 123; Lizard, 176; Pigeon, 254: Rabbit, 366.

Membranous labyrinth, see Auditory organ.

Membrana semilunaris:-Pigeon, 246.

Meniscus of jaw :-Rabbit, 276. of vertebræ:-Pigeon, 186.

Mesencephalon, see Mid-brain.

Mesenteric bands:-Lamprey, 15.

Mesentery:-Cod, I12; Lizard, 164: Pigeon, 223; Rabbit, 297.

Mesethmoid:-Cod, 94: Lizard, 143; Pigeon, 191, 197; Rabbit, 273, 336.

Mesoarium:-Lamprey, 14 ; Skate, 54; - Cod, Ir2; Lizard, r68.

Mesocole, see under Ventricles of brain.

Mesogaster:-Skate, 47 ; Cod, I12; Lizard, 164; Pigeon, 223; Rabbit, 297.

Mesohepar:-Lizard, 164.

Mesopterygium:-Skate, 39 .

Mesopterygoid:-Cod, 96.

Mescrchium:-Lamprey, 14; Skate, 54; Cod, 112; Lizard, 166; Rabbit, 319.

Mesorectum:-Skate, 47; Cod, II2 ; Lizard, 164 ; Pigeon, 223; Rabbit, 297.

Metacarpus, see Manus, skeleton of.

Metapterygoid:-Cod, 96 .

Metatarsus, see Pes, skeleton of.

Mid-brain or Mesencephalon, see Optic lobes.

Mouth, aperture of, see External characters.

cavity of:-Lamprey, 20; Skate, 65 ; Cod, 102: Lizard, 175; Pigeon, 248 ; Rabbit, 333 .

Müllerian duct, see Pronephric duct.

Muscles of eye:-Skate, 77; Pigeon, 249; Rabbit, 329.

of fore limb:-Pigeon, 25I ; Rabbit, 339.

of hind limb :-Pigeon, 252; Rabbit, $35 \mathrm{x}$.

of larynx:-Rabbit, 337.

of trunk:-Lamprey, II, I3 ; Cod, 106; Lizard, 156; Pigeon, 215; Rabbit, $29 \mathrm{r}$.

Muscles-

abductor brachii inferior:-Rabbit, 344.

brachii superior: - Rabbit, 343 .

minimi digiti : $-R$ abbit, 350 .

pollicis :-Rabbit, 348 . 
Muscles (continued) -

brevis:-Rabbit, 358 .

longus: - Rabbit, 357 ,

magnus :-Rabbit, 357 .

ambiens:-Pigeon, 254.

anconeus, see triceps.

arytenoid :- Rabbit, 337

biceps brachit:-Pigeon, 251; Rabbit, 346.

biceps femoris : $-R$ abbit, 356 .

basio-humeralis:-Rabbit, 34I.

brachialis internus: $-R a b b i t, 34$.

bursalis:-Pigeon, 249.

cleido-mast oid: $-R a b b i t, 34 \mathrm{I}$.

coraco-brachialis: $-R a b b \imath t, 344$.

coraco brachialis longus:-Pigeon, 218.

costo-pulmonary:-Pigeon, 244.

cremaster:-Rabbit, 318.

crico-arytenoid:-Rabbit, 337, 338.

crico-thyroid:-Rabbit, 337 .

crureus:-Rabbit, 356 .

deltoid:-Rabbit, $34 \mathrm{I}$.

erector spinæ:-Rabbit, 343

extensor carpi radialis :-Rabbit, 346 . ulnaris: - Rabbit, 348 .

communis digiturum (manus):Rabbit, 46 .

communis digitorum (pedis):Rabbit, 360 .

digiti primi (pedis): $-\pi$ abbit, 360 . hallucis brevis:-Pigeon, 252 .

longus digitorum (pedis):-Pigeon, 252.

metacarpi radialis longus:- $P$ igeon, $25 \mathrm{I}$.

parvus antibrachii : - Rabbit, 345 .

pollicis et indicis :-Rabbit, 348 .

quarti digiti : - Rabbit, 348 .

quinti dig.ti : - Rabbit, 348 .

flexor brevis minimi digiti $:-R$ abbit, 350.

pollicis : - Rabbit, 350 .

carpi radialis: $-R a b b i t, 348$. ulnaris:-Rablit, 348 .

digitorum communis (pedis):Rabbit, $36_{3}$.

digitorum profundus $v$. perforans: -Pigeon, 253 : Rabbit, 349 .

digitorum sublimis $v$ perforatus : -Pigeon, 253; Rabbit, 34 .

gastrocnemius:-Pigeon, 253 ; Kabbit, 360.

gemelli : - Rabbit, 359 .

glutei (maximus, medius, and mini-

mus) : - Rabbit, 358, 359 .

gracilis :- Rabbit, 354 .

iliacus:-Rabbic, $353,358$.

infra-spinatus: - Rablit, 344 .

intercostals:-Lizard, I59; Rabbit, 290.

interossei (manus): - Rabbit, 35r. (pedis): - Rabbit, $36+$.
Muscles (continued)-

latissinus dorsi :-Rabbit, 339.

levator anguli scapulæ: - $R$ abbit, 343 . scapulæ major:-Rabbit, 34r.

lumbricales (manus): $-R$ abbit, 350. (pedis): - Rabbit, 363 .

masseter:-Rabbit, 329 .

mylohyoid:-Lizard, ${ }_{5} 8$.

oblique of abdomen (external and internal):-Lizard, 158, I59; Pigeon, 216; Rabbit, $2 \ni$ I.

oblique of eye (superior and inferior):Skute, 77. 78 ; Rabbit, 329, 330.

obturator (internus and externus):Ŕabbit, 359 .

palmaris: $-K a b b i t, 349$.

panniculus carnosus:- $-R$ abbit, 288.

papillary, see Heart, dissection of.

pectınate, see Heart, dissection of.

pectineus :- Rabbit, $35^{8}$.

pectoralis :-Lizard, 158.

major:-Pigeon, 215, 252 ; Rabuit, 290.

minor:-Rabbit, 290, 343 .

secundus, see subclavius.

tenuis :-Rabbit, 290.

peroneus brevis: - Rabbit, $3_{6}{ }_{3}$.

longus:-Rabbit, $3^{6}{ }_{3}$.

medius:-Pigeon, 254 .

quarti digiti :-Rabbit, $3^{6} 3$.

quinti digiti : - Rabbit, 363 .

plantaris:-liabbit, 362 .

popliteus:-Rabbit, 362 .

pronator teres:-Rabbit, 348.

psoas magnus:-Rabbit, 351, 358 . parvus:- Rabbit, 353 .

pterygoid:-kabbit, 332 .

pyramidalis:-Piseon, 249 .

pyriformis : $-R a b b i t, 35$.

quadratis femoris :-Kabbit, 359 .

lumburum:-Rabbit, 353 .

quadriceps: $-R$ abbit, 354 .

rectus abdominis:-Lizard, 156; $P$ igeon, $216 ;$ Rabbit, 291.

anticus:-Rabbit, 354,356 .

recti of eye:-Skate, 77,$78 ;$ Rabbit, 330.

retractor bulbi :-Rabbit, 330 .

of tongue :-Lamprey, 18.

rhomboideus cervi $: a l i s:-K a b b i t, 342$. dorsal $_{\mathrm{s}}$ :-Rabbit, 342 .

sartorius :-Rabbit, 354 .

semi-membranosus:- - Rabbit, 357.

semi-tendinosus:- Rabbit, 357.

serratus magnus:-Rabbit, 343 .

soleus:-Rabbit, 362 .

stapedius :-Rabbit, 332 .

sterno-mastoid:-Rabbit, 290.

sterno-tracheal:-Pigeon, 221.

subclavius:-Pigeon, 218, 252.

subscapularis :-Rabbit. 344 .

supra-spinatus:-Rabbit, 344 .

syringeal, intrinsic:-Piseon, 244 . 
Muscles (continued)-

tensor patagii, longus, brevis, and, accessorius :- Higeon, 25I, 252.

tympani :-Rabbit, 332 .

teres major:-Rabbit, 344 .

minor:-Rabbit, 345 .

thyro-arytenoid:-Rabbit, 338 .

tubialis anticus :- $R$ abbit, 360 .

transversalis abdominis:- Higeon,216; Rabbit, 29 .

trapezius:-Rabbit, 339 .

triceps:-Pigeon, 25I; Rabbit, 345.

vastus internus and externus:- - kabtit, 354,356 .

Myelencephalon, see Medulla oblongata.

Myelocœle, see Ventricles of brain, 4th.

Nlyelon, see Spinal cord.

Myotomes or Myoccmmas:-Lamprey, 12; Cod, 106.

N.

NARES, anterior, see External characters. posterior, see Mouth cavity.

Nasal tones:-Cod, 99: Lizard, 138, Pigeon, 197; Rabbit, 274.

cæcum:-Lamprey, 25.

capsule:-Lamprey, 5; Skate, 35 ; Lizard, 143.

sac, see Ulfactory organ.

Naso-buccal groove:-Skiate, 42.

Naso-palatine canal :-Lamprey, 4; Rabbit, 335 .

Nerves-

Ist cerebral (olfactory):-Lamprey, 24 ; Skate, 72, 79; Cod, 124; Lizard, 178 ; Pigeon, 256; Káubit, 336, 368. and cerebral (optic):-Lamprey, 24; Skate, 73, 78; Cod, 126; Lizard, 179; Pigeon, 256; liabbit, 330, 369 .

3rd cerebral (oculomotor):-Lamprey, 24; Skate, 74, 77, 78; Cod, I 26; Lizard, 179; Pigeon, 256;.Rabbit, 330,369 .

$4^{\text {th }}$ cerebral (pathetic):-Lamprey, 25 ; Skate, 74, 77 ; Cod, 126; Lizard, 179 ; Pigeon, 257 ; Rablit, 330, 369.

5th cerebral (trigeminal):-Lamprey, 25 ; Skate, 75-79; Cod, 106, I26; Lizard, 179; Pigeon, 257; Rabbit, 332, 369.

6th cerebral (abducent):-Skate, 76 , 77; Cod, 127 : Lizard, 179; Pigeon, 257 ; Rabbit, 330, 369 .

7 th cerebral (facial or portio dura):Lamprey, 25; Skate, 76, 80; Cod, 122, 126; Lizard, 179; Pigeon, 257 ; Rabbit, 329, 370.

8th cerebral (auditory):-Lamprey, 25 ; Skate, 76, 82 ; Cod, 127 ; Lizard, 179; Yigeon, 257; Rabbit, 370.
Nerves (continued) -

$9^{\text {th }}$ cerebral (glossopharyngeal) :Lamprey, 25; Skate, 76, 82, 83; Cod, 119, 127 ; Lizard, x79; Pigeon, 257; Rabbit, 332, 370.

toth cerebral (vagus or pneumogastric):-Lamprey, 25 ; Skate, 51, 76, 81,83 ; Coul, Iс6, I12, I19, Ig6; Lizard, 179; Pigeon, 225, 240, 257 ; Rabbit, 299, 311, 313, 332, 370 .

IIth cerebral (accessory):-Lizard, 179; Pigeon, 257 ; Rabbit, 332, 370. I th cerebral (hypoglossal):-Lizard, 179: Figeon, 257; Ralbit, 313, $33^{2}, 370$.

branchial:-Skate, 82.

circumflex:-Rabbit, 338 .

chorda tympani:-Skate, 80 ; Cod, 126. crural :-Rabbit, 339.

cutaneous:-Cod, 106, 127.

femoral:-Pigeon, 247.

gustatory:-Kabbit, 332.

hyomandibular:-Skate, 8o.

mandibular:-Skate, 79; Cod, 126; Rabbit, 332.

maxillary:-Skate, 79; Cod, 126; Rabbit, 333 .

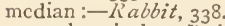

musculo-spiral :-Kabbit, 338.

orbito-nasal:-Skate, 76-78; $\mathrm{Cod}$, 126 ; Ralbit, 330.

obturator:-Pigeon, 247 ; Rabbit, 339.

palaine:-Skate, 80.

palato-nasal:-Skate, 79; Cod, 126.

phrenic:-Rabbit, 3 II.

sciatic:-Pigeon, 247 ; Rabbit, 339.

spinal:-Skate, 82, 83; Pigcon, 247.

splanchnic:-Pigeon, 226; Kabbit, 299.

subscapular:-Kabbit, 338 .

sympathetic:-Skate, 65, 83; Cod, I16; Pigeon, 247; Rabbit, 312, วิ13. ulnar:-Kabbit, 338 .

Nerve-plexus-

brachial:-Skate, 82; Pigeon, 247; Rabbit, 338.

lumbar:-Yigeon, 247.

lumbo-sacral:- Rabbit, 338.

sciatic:-Pigeon, 247.

Neural canal and processes:-Lamṕrey, 2. arch, processes, and spine, seeVertebra.

Neurapophysis, sce Vertebra.

Nictitating membrane, see External characters.

Nostrils, see External characters.

Notochord:-Lamtrey, 2.

O.

Oblique septum:-Pigeon, 226.

Occipital arch:-Lamprey, 5. bone, see Basi-, Ex-, and Supra-occipital. 
Occipital (coutinued)-

condyle:-Skate, 34; Cod, 91; Lizard, $136 ;$ Piyeon, 19o; Rabbit, 268. foramen, see Foramen magnum.

Odontoid bone or process:-Skate, 29; Lizard, I33; Pigeon, 185; Rabblit, 267.

CEsophagus, see Gullet.

Olfactory foramen, see under Foramen.

lobe:-Lamprey: 24 ; Skate, 72, 79; Cod, 124; Lizard, 178; Pigeon, 256; Rabbit, 363.

organ:-Lamprey, 24; Skate, 78, 79: Cod, 122; Pigeon, 249; Rabbit, 335 .

Omentum, duodeno-hepatic:-skate, 47 ; Lizard, 164; Pigeon, 222; R'abbit, 297.

gastro-hepatic:-Skate, 47 ; Cod, Ir2 ; Lizard, 164; Pigeon, 223 ; Rabbit, 297.

gastro-splenic:-Skate, 47; Rabbit, 297.

great:-Pigeon, 217, 222; Kabbit, 297.

Opercular bones:-Cod, 99 .

() Ferculum:-Cod, roz.

Ophthalmic peduncle:-Skate, 78.

Opisthotic:-Cod, 93; Lizard, I40; Pigeon, 194.

Optic chiasma and tracts:-Skate, 74: Lizard, 179; Pigeon, 256: Rabbit, $369,376$.

commissure :-Pigeon, 258.

foramen, see under Foramen.

lobes:-Lamprey, 25; Skate, 70; Cod, 124; Lizard, 178; Pigeon, 256 ; Rabbit, 376.

thalami:-Lamprey, 23; Skate, 72; Lizard, 180; Pigreon, $25^{8}$; Rabbit, 375 .

Ora serrata, see Eye, dissection of.

Oral aperture, see External characters.

cæcum:-Lamprey, 20.

cavity, see Mouth, cavity of. funnel:-Lamprey, 8, 13 .

Orbit:-Lizard, 135; Pigeon, 190, 191 ; Rabbit, 268.

Orbito-sphenoid:-Rabbit, 27 I.

Os cloacæ:-Lizard, r5o.

innominatum, see Hip-girdle. orbiculare:-Rabbit, 277 .

Ossicula auditûs :-Rabbit, 277 .

Otoliths or otolithic mass:-Skate, $8 \mathrm{I}$; Cod, 123 .

Ovary:-Lamprey, 13; Skate, 54; Cod, 108, I14; Lizard, 167; Pigeon, 229; Rabbit, 322.

Oviducal gland :-Skate, 58.

Oviduct:-Skate, 5i, 56, 58 ; Cod, II 4 ; Lizard, 168, 171 ; Pigeon, 229, 236.
P.

Palatine bone:-Cod, 96 ; Lizard, 141 : Pigeon, 196: Rabbit, 275.

foramen:-Lizard, 136.

Pancreas:-Skate, 44; Lizard, ${ }^{6} 64$; Pigeon, 220; Rabbit, 300.

Pancreatic duct:-Skate, 50; Pigeon, 224 ; Rabbit, 300.

Parachordal plate:-Lamprey, 4

Parasphenoid:-Cod, 94; Lizard, 14I ; Pigeon, 192.

Parethmoid :-Cod, 95; Rabbit, 274.

Parietal:-Cod, 94 ; Lizard, I38; Pigeon, r9o; Rabbit, 270.

Parorchis:-Skate, 56.

Parotic or paroccipital process:-Cod, 93 ; Lizard, 136.

Parovarium:-Skate, 6o.

Patella:-Pigeon, 208; Rabbit, 284.

Pecten of eye :-Pigeon, 250.

Pectoral fin, see Fore limb.

Pelvic fin, see Hind limb.

Pelvis, see Hip-girdle.

Penis:-Lizard, 171; Rabbit, 287, 316 ; 320.

Pericardio-peritoneal canals:-Skate, 64 . septum:-Skate, 45; Cod, rog.

Pericardium and pericardial cavity :Lamprey, 8, 15, 19; Skate, 45; Cod, 109, 110; Lizard, 162; Pigeon, 220; Rabbit, 293, 307.

Perineal gland :-Rabbit, 320 .

Peritoneum:-Lamprey, 13; Skate, 44, 47 ; Cod, 107, 109, 112; Lizard, 15\%: Pigeon, 222 ; Rabbit, 29I, 298.

Pes, see External characters. skeleton of:-Lizard, 152; Pigeon, 208; Rabbit, 285 .

Pessulus:-Pigeon, 246.

Peyer's patches:-Rabbit, 305 .

Phalanges, see Manus, skeleton of, and Pes, skeleton of.

Pharyngo-branchial, see Branchial arch.

Pharynx;-Skate, 65; Cod, 120; Lizard, 175 ; Figeon. 248 ; Rabbit, 333.

Pia mater:-Skate, 46 ; Rabbit, 295.

Pineal body:-Lizard, 178 ; Pigeon, 256 ; Rabbit, 368,375 .

Pituitary body:-Skate, 72 ; Cod, 124: Lizard, 178; Pigeon, 256; Rabbit, 369.

Pleura:-Lizard, 168; Pigeon, 244; Rabbit, 294, 307.

Plexus, see Nerve-plexus.

Pons Varolii :-Rabbit, ${ }_{367}$.

Pori femorales:-Lizard, 156,

Post-clavicle:-Cod, roo.

Post-orbital bones:-Lizard, 139 .

Post-temporal :-Cod, roo.

Poupart's ligament:-Rabbit, 29r.

Precoracoid:-Lizard, 146 .

Pre-frontal:-Lizard, $=39$. 
Pre-maxilla:-Cod, 96: Lizard, 138 : Pigeon, 196; Rabbit, 274.

Pre-opercular, see Upercular bones.

Pre-pubis:-Lizard, 150.

Prepuce:-Rabbit, 316.

Presphenoid:-Rabbit, 27 I.

Processus falciformis of eye: $-\operatorname{Cod}, 122$.

Pronephric duct:-Skate, 52, 56.

Prootic:-Cod, 93; Lizard, 140; Pigeon, I94.

Propterygium :-Skate, 39 .

Prosencephalon, see Cerebral hemispheres.

Prostate :-Ralbit, 319, 321.

Proventriculus:-Pigeon, 225, 227.

Pseudobranchia:-Skate, 43; Cod, Iо3, I20.

Pterotic:-Cod, 93.

Pterygoid bone:-Cod, 96 ; Lizard, 141 : Pigeon, 195; Rabbit, 275.

Pterygo-quadrate cartilage:-Skate, 37.

Pterylæ, or feather tracts: - Pigeon, 2 I I.

Pterylosis, or feather arrangement :Pigeon, 211.

Pubis, see Hip-girdle.

Pupil, see Eye.

Pygostyle:-Pigeon, I 88.

Pylangium, see Heart.

Pyloric cæca:-Cod. 107, II3. valve, see under Valve.

\section{Q.}

QUadrate:-Cod, 96; Lizard, 136, 140; Pigeon, 195.

Quadrato-jugal:-Pigeon, 197.

R.

Radius:-Lizard, 148; Pigeon, 202: Rabbit, 280.

Rectal gland:-Skate, 47 ; Rabbit, 320.

Rectrices:-Pigeon, 212.

Rectum:-Lamprey, 14; Skate, 47 ; Cod, II ; Lizard, 164; Pigeon, 223 ; Rabbit, 292, 305 .

Remiges:-Pigeon, $2 \times 2$.

Reproductive organs, see Testis, Ovary, Sc.

Respiratory tube:-Lamprey, 18, 20.

Restiform bndies, see Medulla oblongata.

Rete mirabile of air-bladder :-Cod, II 5 .

Retina, sce Eye.

Rhinencephalon, see Olfactory lobe.

Ribs:-Skate, 32; Cod, 90 ; Lizard, 135: Pigeon, 185, 186; Rabbit, 268.

Rostrum of skull and rostral cartilages:Skate, 35 .

S.

SAcculus rotundus : $-\mathrm{R} a b b i t, 304$.

Saccus vasculosus:-Skate, 72.

Sacrum, see Vertebral colitmn.
Salivary glands : - Rabbit, 289, 313, 329 . sac:-Lamprey, 19.

Scales:-Skate, $4 \mathrm{r}$; Cod, 101, 102 ; Lizard, 154 .

Scapula, see Shoulder-girdle.

Schneiderian membrane, see Olfactory organ.

Sclerotic, see Eye, dissection of. plates:-Lizard, i76; Pigeon, 250.

Scrotal sacs:-Rabbit, 292, 318.

Sella turcica :-Kabbit, 27 o.

Semicircular canals, see Auditory organ.

Semilunar fibru-cartilages :-Lizard, I52; R'abbit, 285.

Sensory tubes:-Lamprey, Ix; Skate, 43.

Septomaxillary:-Lizard, 145 .

Septum lucidum :-Rabbit, 373 , nasi, see Mesethmoid.

Sesam id bones:-Rabbit, 28r, 282, 284, 286.

Shoulder-girdle:-Skate, $3^{8}$; Cod, 100; Liza)d, 146 ; Pigeon, 200 ; Rabbit, 278.

Sinus (venous, lymphatic, \&c.) cardinal:-Skate, 52 .

circum-œsophageal:-Lamprey', 19.

hepatic:-Skate, 51, 65.

peribranchial :-Lamprey, 18.

precaval:- Skate, 65 .

rhomboidalis:-Pigeon, 222.

spermatic:-Skate, 52.

venosus, see Heart.

Skeleton, preparation of:-Lamprey, 1; Skate, 27; Cod, 86; Lizard, 130; Pigeon, 182; Rabbit, 262.

general characters of:-Skate, 28; Cod, 87; Lizard, 131; Pigeon, 183: Rabbit, 262.

Skull:-Lamprey, 4 ; Skate, 34; Cod,91 ; Lizard, 135: Pigeon, 190; Rabbit, 268.

Specific characters:-Petromyzon, 8, Iо : Raja, 41, 42; Gadus, 91, 100, 102; Lacerta, 155 .

Sperm sacs:- Skate, 56, 59 .

Sphenoidal fissure:- $K$ abbit, 271.

Sphenotic:-Cod, 93.

Spinal cord:-Lamprey, 23; Skate, 32, 7o; Pigeon, 222.

Spiracle:-Skate, 43, 65.

Spiracular cartilage:-Skate, 37 .

Spiral valve, see under Valve.

Spleen:-Skate, 44; Cod, 108; Lizard, 164; Pigeon, 225; Rabbit, 297.

Splenial, see Mandible.

Squamosal:-Lizard, r40; Pigeon, 191 ; Rabbit, 27 .

Stapes:-Lizard, 142; Pigeon, 195: Rabbit, 277.

Stenson's canal, see Naso-palatine canal. duct, see Salivary glands.

Sternebræ: - Rabbit, 268.

Sternum:-Lizard, 134; Pigeon, I98: Rabbit, 268. 
Stomach:-Skate, 44, 47; Cod, 107, III, II3; Lizard, 163, 170; Pigeon, see Gizzard and Proventriculus; Rabbit, 292, 296, 305 .

Strie longitudinales:-Rabbit, 372.

Stylo-hyal, see Hyoid.

Sub-ocular arch :-Lamprey, 5.

Sub-opercular, see Opercular bunes.

Sub-orbitals :-Cod, roo.

Sub-vertebral wedge bone:-Lizard, 133.

Supra-angular, see Mandible.

Supra-clavicle:-Cod, roo.

Supra-occipital:-Cod, 9I ; Lizard, 140 : Pigeon, 190; Rabbit, 259.

Supra-orbitals :-Lizard, 139.

Supra-renal bodies, see Adrenals.

Supra-scapula, see Shoulder-girdle.

Supra-temporal:-Lizard, 139.

Suspensory ligament :-Rabbit, 296.

Sutures, cranial, see Skull.

Symplectic :-Cod, 95 .

Synangium, see Heart.

Synovial cavity:-Pigeon, IE6; Rabbit, 351,364 .

Syrinx:-Pigeon, 244-246.

\section{T.}

TANIA semicircularis:-Rabbit, 374 .

Tapetum:-Rabbit, 33r.

Tarsus, see Pes, skeleton of.

Tarso-metatarsus :-Pigeon, 208

Teeth:-Lamprey, 10; Skate, 42; Cod, 120 ; Lizard, 175; Rabbit, 277, 278.

Tendo Achullis:-Rabbit, 360 .

Tentorium cerebelli, or Tentoral ridge :Pigeon, 193; Rabbit, 295.

Testis:-Lamprey, 13; Skate, 54; Cod, 108, 113; Lizard, 165; Pigeon, 228; Kabbit, 3 ㅇ․

Thalamencephalon:-Laniprey, 23 ; Skate, 72; Cod, 124; Lizard, 179; Pigeon, 258 ; Rabbit, 375 .

Thalami optici, see Thalamencephalon.

Thalamocole, see Ventricles of brain, $3^{\text {rd. }}$

Thoracic duct:-Rabbit, 312 .

Thymus gland:-Skate, 69; Rabbit, 293.

Thyroid cartilage, see Larynx. gland:-Skate, $6_{4} ;$ Lizard, $16_{3} ; \mathrm{Kab}$ bit, 313 .

Tibia :-Lizard, 151; Rabbit, 284.

Tibio-tarsus:-Pigeon, 206.

Tongue, see Mouth cavity.

Tori semicirculares:-Cod, 128.

Trabecule: -Lamprey, 4.

Trachea:-Lizard, 162, 175 ; Pigeon, 215, 220, 244; Rabbit, 289, 325 .

Trans-palatine or Os transversum :Lizard, I4I.

Transverse process, see Vertebra.

Trochanter, see Femur.

Trochlea, see Humerus.
Tuberosity, see Humerus and Tibia.

Turbinals:-Lizard, 145; Pigeon, 197, 249: Rabbit, 274, 275, 335 .

Tympanum or tympanic cavity, and tympanic membrane:-Lizard, 137, 176 ; Pigeon, 192, 245, 250; Rabbit, 272, 331.

U.

Ulna :-Lizard, :48 ; Pigeon, 202 ; Kabbit, 28r.

Uncinate processes:-Pigeon, 185 .

Ureter:-Lamprey, 15; Skate, 59, 60 ; Cod, 10); Lizard, 171; Pigeon,231, 235: Rabbit, 300, 302, 3i6, 320, 323 .

Urethra :-Rabtit, 3 I8.

Urinary aperture, see External characters. bladder, see under Bladder. papilla :-Skate, 5 .

Urinogenital organs, dissection of :-Lamprey, 13; Skate, 54; Cod, I13: Lizari, 165, 171; Pigeon, 228; Raóbit, 316 .

papilla:-Lamprey, 10, I5; Skate, 58; Lizard, 17 1.

sinus:-Lamprey, 15; Skate, 59.

Urohyal, see Hyoid.

Uropygial gland:-Pigeon, 247.

Uterus:-Skate, 56; Rabbit, 321, 324. masculinus :-Rabbit, 319, 321 .

\section{V.}

VAgina :-Rabbit, 321, 323.

Valve, or Valves-

of heart, see Heart, dissection of.

ileo-colic:-Lizard, 170; liabbit, 306. intra-colic:-Rabbit, 306.

pyloric:-Skate, $52 ; \operatorname{Cod}, 113 ;$ Lizard, 170; Pigeon, 227 ; Rabbit, 305.

spiral :-Lamprey, I6; Skate, 52; Rablit (cæcum), зс6.

of Vieussens:-Rabbit, 377 .

Valvulae conniventes:- Lizard, 170 ; Rabbit, 305.

Vas deferens:-Skate, 56, 59; Cod, $11_{4}$; Lizard, 171 ; Pigeon, 228, 236 ; Rabbit, $318,320$.

Vein-

anterior abdominal, see epigastric. azygos, see cardinal.

brachial:-Skate, 65 ; Pigeon, 238. cardinal:-Lamprey, 16, 22; Skate, 52,60, 65; Cod, II5, I16; Lizard, 165: Rablit, 312.

caudal:-Lamprey, 17; Skate, 67; Cod, I16; Lizard, 169;. Pigeon, 231.

coccygeo-mesenteric:-Pigecn,225,231. corcnary :-Rabbit, 327 . 
Vein (continued)-

epigastric:-Skate, 68; Lizard, 161 ; Pigeon, 22I, 240; Rabbit, 315.

femoral:-Skate, 68; Lizard, 169: Pigeon, 23x ; Rabbit, 315.

gastric:-Skate, 50; Lizard, 165; Pigeon, 224.

gastru-duodenal :-Pigeon, 224.

hepatic:-Lamprey, 22 ; Skate. 51; Cod, 113 ; Pigeon, 240 ; Rabbit, 306. hypogastric, see renal portal.

iliac (common, internal, and external) : -Pigeon, 230: Rabbit, 315.

ilio-hænorrhoidal :-Skate, 68.

intercostal :-Rabbit, 3I2.

jugular (external, internal, and inferior):-Lamprey, 21, 22: Skate, 63,$65 ; \operatorname{Cod}, 115,118 ;$ Lizard, 163 ; Pigeon, 238, 239: Ralbit, 289, 310.

mammary :-Pigeon, 238.

mesenteric (anterior or superior, and posterior or inferior):-Skate. 50 ; Cod, r12; Lizard, r65; Pigeon, 224; Rabbit, 3 co.

pancreatic:-Lizard, $16_{5}$.

pectoral :-Pigeon, 238.

pelvic:-Lizard, 161, 169; Pigeon, $23 \mathrm{r}$.

phrenic (anterior and posterior):Rabbit. 307. 3II.

portal:-Skate, 49, 50; Cod, 112; Lizard, 165; Pigeon, 224: Rabbit, 303.

precaval (vena cava superior s. anterior):-Cod, II5; Lizard, I63, 172; Pigeon, 238, 240; Rabbit, 310 , 326.

postcaval (vena cava inferior s. posteri r) :-Lizard, 162, 168, 169, 172; Pigeon, 230, 239; Rabbit, 298, 306, $31 \mathrm{I}, 326$.

pulmonary:-Lizard, 173; Pigeon, 240 ; Rabbit, 308. 325, 327.

renal:-Lizard, 169; Pigeon, 233; Rabbit, 3 I4.

afferent:-Pigeon, 234 .

efferent:-Pigeon, 233 .

portal:-Skate, 67; Lizard, I69; Pigeon, 231, 234.

sciatic:-l.izard, 169; Pigeon, 234.

spermatic:-Cod, I13; Rabbit, 314.

splenic :-Lizard, 165 .
Vein (contimued)-

subcluvian:-Rabbit, 3 Io.

vertebral :-Pigeon, 239.

Velum interpositum:-Pis'Eon, 258; Rabbit, 375 .

palati, see Mouth cavity.

Ventricles of heart, see Heart.

larynx, see Larynx.

brain-

$3^{\text {rd : }}$-Lamprey, 23; Skate, 72:

Cod, 129; Lizard, 181; Pigeon 258, $260 ;$ Rabbit, 375 .

4th:-Lamprey, 23; Skate, 73 ;

Co.d, 128 ; Lizard, 178 ; Pigeon, 254, $260 ;$ Rabbit, 376.

5th:-Rabbit, 373 .

lateral:-Lizard, r8o ; Pigeon, 258 ; Rabbit, 372,374 .

olfactory :-Lizard, I8o.

optic:-Lamprey, 23: Skate, 73 : Cod, 123; Lizard, 180; Pigron, 260 .

Vertebra, characters of, and Vertebral culumns:-Lamprey, 2 ; Skate, 29 ; Cod. 88; Lizard, 132; Pigeon, 183; Rabbit, 265 .

Vertebrarterial canal:-Pigeon, $185 ;$ Rabbit, 266.

Verumontanum :-Rabbit, 321.

Vesicula seminales:-Skate, 59.

Vestibule, see Auditory organ.

(female urinogenital canal):-Rabbit, $321,323$.

Villi :-Pigeon, 227; Rabbit, 305.

Vitreous humour, see Eye.

Vocal chords, see Larynx.

Vomer:-Cod, 94; Lizard, 141; Pigeon, 196; Rabbit. 274.

Vulva :-Rabbit, 321 .

W.

WING, see Fore limb.

$\mathrm{X}$.

XIPHOID process, see Sternum.

Z.

ZyGAPOPHYSES, see Vertebra.

THE END. 



\section{MANUALS FOR STUDENTS.}

PHYSIOGRAPHY. An Introduction to the Study of Nature. By Professor HUXLEY, P.R.S. With Illustrations. Crown $8 \mathrm{v}$ ). $6 s$.

HEAT. By Professor P. G. TaIt M.A., Sec. R.S.E. With Illustrations. Crown 8vo.

ANTHROPOLOGY. An Introduction to the Study of days. and Civilisation. By E. P TYLOR, D.C. F.R.S Crown

A COURSE OF ELEMENTARY PRACTICAL PHYSIOLOGY. By Professor MICHAEL FOSTER, M.D.. F.R.S., \&c., and J. N. LANGLEY, F.R.S. New Edition. Crown 8vo. 7s. $6 d$.

THE ELEMENTS OF EMBRYOLOGY. By Professor MICHAEL FOSTER, M.A., M.D., LL.D., F.R.S., and the late FRANCIS M. BALFOUR. M.A., LL D., F.R.S. Second Edition, revised. Edited by Adam Sedgwick, M.A. With Illustrations. Crown 8vo. ros. $6 d$.

A COURSE OF PRACTICAL INSTRUCTION IN ELEMENTARY BIOLOGY. By Professor HUXLEY, P.R.S., assisted by H. N. MARTIN, M.B., D.Sc. New Edition, revised. Crown 8vo. $6 s$.

TABLES OF MATERIA MEDICA: A Companion to the Materia Medica Museum. By T. LAUDER BRUNTON, M.D., F.R.S. With Illustrations. New Edition, enlarged. 8vo. ros. 6 d.

THE MORPHOLOGY OF THE SKULL. By Professor PARKER and G. T. BETTANY. Illustrated. Crown 8vo. Ios. $6 d$.

THE STUDENT'S FLORA OF THE BRITISH ISLANDS. By Sir J. D. HOOKER, K.C.S.I., C.B., M.D., F.R.S., D.C.L. Globe 8 vo. ros. 6 d.

STRUCTURAL BOTANY, OR ORGANOGRAPHY ON THE BASIS OF MIORPHOLOGY. To which are added the principles of Taxonomy and Phycography, and a Glossary of Botanical Terms. By Professor ASA GRAY, LL.1). 8vo. Ios. $6 d$.

AGRICULTURAL CHEMICAL ANALYSIS. A Handbook of. By PERCY FARADAY FRANKLAND, Ph.D., B.Sc., F.C.S. Founded upon Leitfaden fïr die Agricultur-Chemische Analy'se, von Dr. F. KROCKER. Crown 8vo. 7s. $6 d$.

THE PRINCIPLES OF SCIENCE. A Treatise on Logic and Scientific Method. By W. STANLEY JEVONS, LL.D., F.R.S. Crown 8 vo. $12 s .6 d$.

STUDiES IN DEDUCTIVE LOGIC. By W. Stanley JEVONS. M.A., LL.D., F.R.S. Crown 8vo. 6s.

A MANUAL OF POLITICAL ECONOMY. By Right. Hon. HENRY FAWCETT, MI.P., F.R.S. Sixth Edition, revised, with a chapter on "State Socialism and the Nationalisation of the Land," and an Index. Crown 8vo. I2s.

THE PRINCIPLES OF POLITICAL ECONOMY. By Professor HENRY SIDGWICK, M.A. 8vo. i6s.

POLITICAL ECONOMY. By FRANCIS A. WALKER, M.A., Ph.D., Author of "The Wages Question," "Money," \&c. 8vo. ros. $6 d$.

TREATISE ON MARINE SURVEYING. Prepared for the use of younger Naval Officers. With Questions for Examinations and Exercises principally from the Papers of the Royal Naval College. With the results. By Rev. JOHN L. ROBINSON. Illustrated. Cr. 8vo. 7 s. $6 \boldsymbol{d}$. 


\section{MESSRS. MACMILLAN AND CO.'S}

\section{TEXT-BOOKS FOR STUDENTS.}

THE PRINCIPLES OF PHYSICS, A TEXT-BOOK OF. By ALFRED DANIELL, M.A., Lecturer on Physics in the School of Medicine, Edinburgh. With Illustrations. Medium 8vo. 2is.

CHEMISTRY. A complete Treatise on Inorganic and Organic Chemistry. By Professor H. E. ROSCOE, F.R.S., and Professor C. SCHORLEM MER, F.R.S. With numerous Illustrations. Medium 8vo.

Vols. I. and II.-INORGANIC CHEMISTRY.

Vol. I.-The Non-Metallic Elements. 2Is. Vol. II. Part I.-Metals. Iss. Vol. II. Part II.-Metals. I8s.

Vol. III.-ORGANIC CHEMISTRY. Part I.-THE CHEMISTRY OF THE HYDROCARBONS and the Derivatives, or ORGANIC CHEMISTRY. With numerous Illustrations. Medium 8vo. 2Is. [PART II. in the press.

A MANUAL OF THE CHEMISTRY OF THE CARBON COMPOUNDS, OR ORGANIC CHEMISTRY. By C. SCHORLEMMER, F.R.S., Professor of Chemistry in the Victoria University the Owens College, Manchester. With Illustrations. 8vo. 14s.

TEXT-BOOK OF GEOLOGY. By ARchibald Geikie, F.R.S. With numerous Illustrations. 8 vo. 28 s.

A TEXT-BOOK OF THE PHYSIOLOGICAL CHEMISTRY OF THE ANIMAL BODY. Including an Account of the Chemical Changes occurring in Disease. By Professor A. GAMGEE, M.D., F.R.S. 2 Vols. 8vo. With Illustrations. Vol. I. x8s.

[VoL. II. in the press.

ELEMENTS OF COMPARATIVE ANATOMY. By Professor CARL GEGENBAUR. A Translation by F. JEFFrey BeLL, B.A. Revised with Preface by Professor E. RaY LANkEster, F.R.S. With numerous Illustrations. 8vo. 2Is.

A TEXT-BOOK OF PHYSIOLOGY. By Professor MiCHAEL FOSTER, F.R.S. With Illustrations. Fourth Edition, Revised. 8vo. 2rs.

TEXT-BOOK OF PATHOLOGICAL ANATOMY AND PATHOGENESIS. By Professor ERNST ZIEGLER of Tübingen. Translated and Edited for English Students by Donald Macalister, M.A., M.B., B.Sc., Fellow of St. John's College. Cambridge. With numerous Illustrations. Medium 8vo. Part I. - GENERAL PATHOLOGICAL ANATOMY. 12s. 6 d.

[PART II. in the press.

A TREATISE ON COMPARATIVE EMBRYOLOGY. By F. M. BALFOUR, M.A., F.R.S., Fellow and Lecturer of Trinity College, Cambridge. With Illustrations. In 2 Vuls. 8vo. Vol. I., 18s. Vol. II., 21s.

THE FERTILISATION OF FLOWERS. By Professor HERMANN MÜLLER. Translated and Edited by D'ARCY W. Thompson, B.A., Scholar of Trinity College, Cambridge. With a Preface by CHARLeS DARWin, F.R.S. With numerous Illustrations. Medium 8vo. 2IS.

POPULAR ASTRONOMY. By S. NEwCOMB, LL.D., Professor U.S. Naval Observatory. With 112 Illustrations and 5 Maps of the Stars. Second Edition, Revised. 8vo. 18s.

$$
\text { MACMILLAN AND CO., LONDON. }
$$






$$
3305
$$


